\title{
Biomarkers of Alzheimer's Disease
}

Citation for published version (APA):

Bos, I. (2018). Biomarkers of Alzheimer's Disease: relations with vascular factors and cognition in the predementia stages. [Doctoral Thesis, Maastricht University]. Proefschriftmaken.nl II Uitgeverij Boxpress. https://doi.org/10.26481/dis.20181012ib

Document status and date:

Published: 01/01/2018

DOI:

10.26481/dis.20181012ib

Document Version:

Publisher's PDF, also known as Version of record

\section{Please check the document version of this publication:}

- A submitted manuscript is the version of the article upon submission and before peer-review. There can be important differences between the submitted version and the official published version of record.

People interested in the research are advised to contact the author for the final version of the publication, or visit the DOI to the publisher's website.

- The final author version and the galley proof are versions of the publication after peer review.

- The final published version features the final layout of the paper including the volume, issue and page numbers.

Link to publication

\footnotetext{
General rights rights.

- You may freely distribute the URL identifying the publication in the public portal. please follow below link for the End User Agreement:

www.umlib.nl/taverne-license

Take down policy

If you believe that this document breaches copyright please contact us at:

repository@maastrichtuniversity.nl

providing details and we will investigate your claim.
}

Copyright and moral rights for the publications made accessible in the public portal are retained by the authors and/or other copyright owners and it is a condition of accessing publications that users recognise and abide by the legal requirements associated with these

- Users may download and print one copy of any publication from the public portal for the purpose of private study or research.

- You may not further distribute the material or use it for any profit-making activity or commercial gain

If the publication is distributed under the terms of Article $25 \mathrm{fa}$ of the Dutch Copyright Act, indicated by the "Taverne" license above, 


\section{Biomarkers of Alzheimer's Disease}

\section{Relations with vascular factors and cognition in the pre-dementia stages}

Isabelle Bos 
(C) Isabelle Bos, Maastricht 2018

All rights reserved. No part of this book may be reproduced or transmitted in any form or by any means, without prior permission in writing by the author, or when appropriate, by the publishers of the publication.

Cover design: Karen Ebert || www.karenebert.com

Lay-out \& printing: ProefschriftMaken || www.proefschriftenmaken.nl

ISBN: 978-94-93019-14-0 


\title{
Biomarkers of Alzheimer's Disease
}

\section{Relations with vascular factors and cognition in the pre-dementia stages}

\author{
PROEFSCHRIFT \\ Ter verkrijging van de graad van doctor aan de Universiteit Maastricht, \\ op gezag van de Rector Magnificus Prof. dr. Rianne M. Letschert, \\ volgens het besluit van het College van Decanen, \\ in het openbaar te verdedigen op \\ vrijdag 12 oktober 2018 \\ om 12.00 uur. \\ door \\ Isabelle Bos \\ geboren op 26 april 1990 te Groningen
}




\section{Promotoren}

Dr. P.J. Visser

Prof. dr. F.J.R. Verhey

\section{Co-promotor}

Dr. S.J.B. Vos

\section{Beoordelingscommissie}

Prof. dr. B.P.F. Rutten, voorzitter

Prof. dr. C.D.A. Stehouwer

Prof. dr. K. Reetz (University of Aachen)

Dr. M. Kester (VUmc)

Dr. S. Köhler

The research described in this thesis was performed at the School for Mental Health and Neuroscience, Department of Psychiatry \& Neuropsychology, Maastricht University, the Netherlands; and the department of Neurology, Washington University School of Medicine, St. Louis, USA; and was financially supported by Innovative Medicine Initiatives (IMI), ZonMW and Alzheimer Nederland.

Printing of this thesis was kindly supported by Alzheimer Nederland and Maastricht University. In addition, financial support by the Dutch Heart Foundation for the publication of this thesis is gratefully acknowledged.

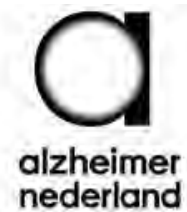


"But time is contagious

and everybody's getting old"

(Damien Rice, 2006) 



\section{Contents}

Chapter 1 General introduction, thesis aims and outline

Part I European Medical Information Framework for Alzheimer's disease

Chapter 2 European Medical Information Framework for Alzheimer's disease

(EMIF-AD): An infrastructure to reuse and combine existing data

Chapter 3 The EMIF-AD Multimodal Biomarker Discovery Study: Design, methods and cohort characteristics

Alzheimer's Research and Therapy, 2018

\section{Part II Vascular contributions to Alzheimer's disease pathology}

Chapter 4 The frequency and influence of dementia risk factors in prodromal

Alzheimer's disease

Neurobiology of Aging, 2017

Chapter 5 Vascular risk factors are associated with cerebrospinal fluid biomarker 83 and cognitive changes in preclinical Alzheimer's disease

Submitted

Chapter 6 Cerebrovascular and amyloid pathology in the pre-dementia stages: the relationship with neurodegeneration and cognitive decline Alzheimer's Research and Therapy, 2017

\section{Part III Established and novel cerebrospinal fluid biomarkers}

Chapter 7 Amyloid- $\beta$, tau and cognition in cognitively normal individuals:

Examining the necessity to adjust for biomarker status in normative data.

Frontiers in Aging Neuroscience, 2018

Chapter 8 Cerebrospinal fluid biomarkers of neurodegeneration, synaptic integrity and astroglial activation in relation to amyloid- $\beta$, APOE genotype and cognition across the clinical Alzheimer's disease spectrum Submitted 
Chapter 9 Increased cerebrospinal fluid amyloid- $\beta 38$ and 40 concentrations

in individuals with mild cognitive impairment and Suspected

Non-Alzheimer's Disease Pathophysiology

Submitted

Chapter 10 General Discussion

Addendum Summary

208

Nederlandse samenvatting

211

Knowledge valorization

214

Author affiliations

217

List of publications

226

Author information

228

Dankwoord

229

Thesis defenses from MHeNs

233 



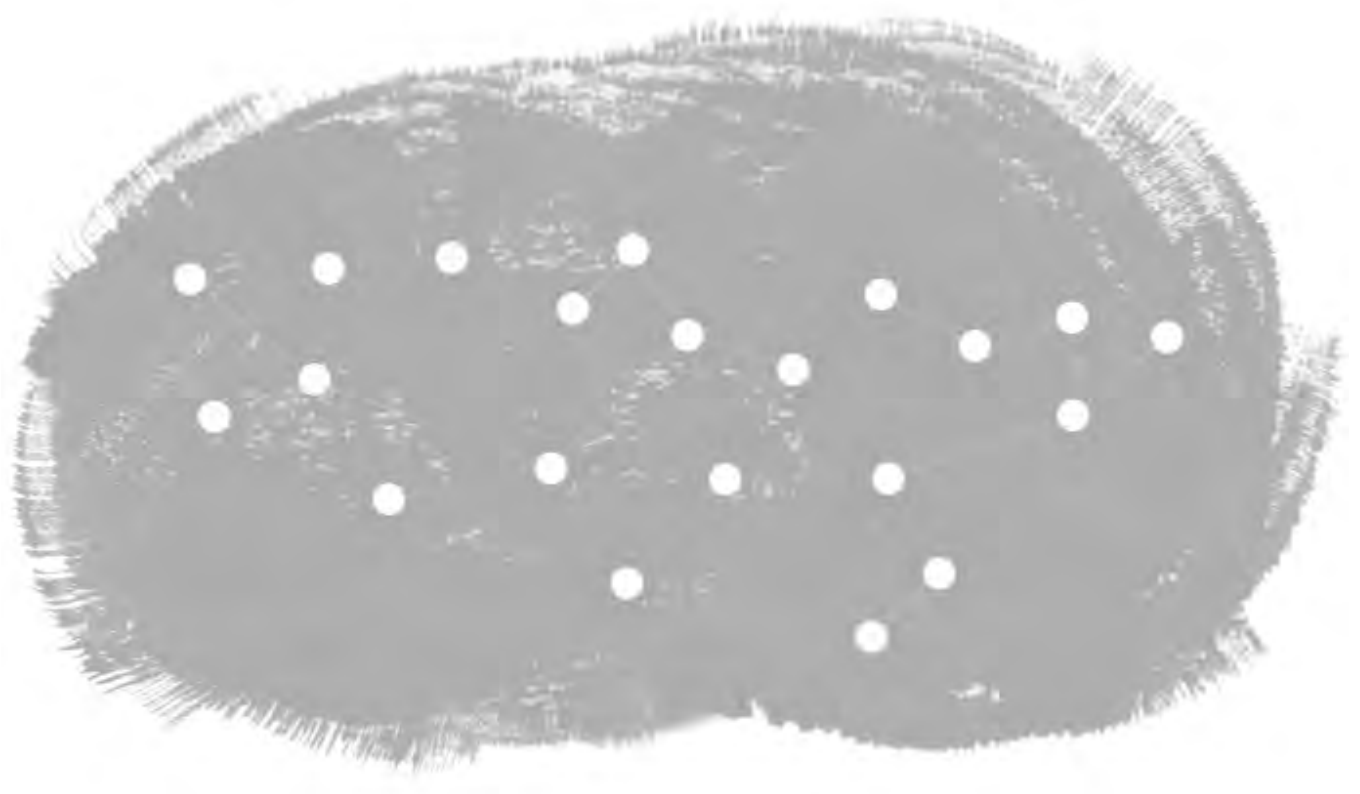




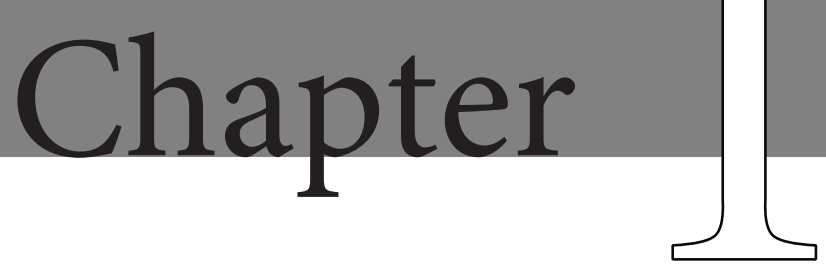

General introduction, thesis aims and outline 


\section{GENERAL INTRODUCTION}

Alzheimer's disease (AD) is the most common cause of dementia, a devastating and complex disease for which currently no effective treatment exists. Besides the well-established pathological hallmarks of $\mathrm{AD}$, amyloid- $\beta(\mathrm{A} \beta)$ plaques and neurofibrillary tangles, additional pathophysiological mechanisms are thought to influence the development and course of $\mathrm{AD}$, but their specific role remains uncertain. An interrelationship between cerebrovascular pathology and $\mathrm{AD}$ has been suggested, ${ }^{1-3}$ as both conditions share common risk factors, are both considered contributors to cognitive decline and neuronal injury, and often co-exist in older individuals. Additional candidate AD mechanisms, which have also been associated with other neurodegenerative disorders, are synaptic dysfunction, ${ }^{4}$ axonal degeneration, ${ }^{5}$ and astroglial activation. ${ }^{6}$ In this thesis, we describe studies conducted to increase understanding of these pathophysiological mechanisms possibly involved in the development and course of $\mathrm{AD}$. This may provide new targets for prevention and treatment strategies, and could increase prognostic accuracy.

\section{Clinical stages of $A D$}

Like many other chronic diseases, pathophysiological changes related to AD begin prior to clinical manifestations of the disease. In general, the clinical course of AD is characterized by three disease stages. During the first stage, the preclinical phase, there are changes in the brain but no clinical symptoms are evident yet. ${ }^{7}$ After the preclinical phase, the prodromal phase is recognized. ${ }^{8}$ During this stage, cognitive functioning has become impaired, referred to as mild cognitive impairment (MCI). ${ }^{9}$ In the dementia phase, the final disease phase, there are cognitive and functional impairments which interfere with a person's daily activities and are gradually becoming worse. ${ }^{10}$ Overall, the preclinical phase can precede the dementia phase by more than 20 years. ${ }^{11,12}$

\section{AD pathology and biomarkers}

\section{Established AD biomarkers}

Where in the past the neuropathological features of AD could only be assessed post-mortem, now biomarkers allow for a clinical diagnosis of AD during life, as they reflect $\mathrm{AD}$ pathology in vivo. ${ }^{13,14} \mathrm{AD}$ pathology is characterized by extracellular amyloid plaques, containing aggregated $A \beta$ proteins, and intracellular neurofibrillary tangles consisting of abnormally phosphorylated tau proteins. ${ }^{13,15}$ Amyloid aggregation is thought to be a primary event in $\mathrm{AD}$ pathogenesis, ${ }^{16,17}$ and can be measured in vivo by a decrease of amyloid-beta (A $\beta$ ) 1-42 protein in the cerebrospinal fluid (CSF) or by an increased retention of $A \beta$ tracer via positron emission tomography (PET) scanning. Formation of neurofibrillary tangles is 
suggested to follow amyloid aggregation, ${ }^{17}$ and can be assessed by tau PET scan or via phosphorylated tau (p-tau) levels in CSF. Other downstream neuropathological changes characteristic for $\mathrm{AD}$ are neurodegenerative processes including axonal damage, cell death and brain atrophy ${ }^{17,18}$, which can be measured by total tau (t-tau) in CSF, hypometabolism on a FDG PET scan, or medial temporal lobe atrophy (MTA) on magnetic resonance imaging (MRI). ${ }^{8}$ Although amyloid aggregation had been hypothesized to be the earliest event in $\mathrm{AD},{ }^{17}$ more than a quarter of individuals with $\mathrm{MCI}$ have $\mathrm{AD}$-like neurodegeneration but no evidence of amyloid pathology, a biomarker-based subtype which has been referred to as Suspected Non-Alzheimer's Disease Pathophysiology (SNAP). ${ }^{19}$ As a substantial number of individuals with MCI-SNAP do progress to AD-type dementia, ${ }^{19}$ it is also important to unravel the characteristics of this biomarker-based subtype.

\section{Novel AD biomarkers}

Over the last years, CSF markers Neurofilament-light (NFL), Neurogranin (Ng) and YKL40 (also known as Chitinase 3-like 1), have evolved as promising AD biomarkers. Still, uncertainties remain regarding their exact role in $\mathrm{AD}$ pathophysiology. ${ }^{20-22}$ High concentrations of NFL have been associated with axonal degeneration to, predominantly, subcortical brain areas, ${ }^{21,23}$ while high YKL-40 levels are thought to reflect astroglial activation, a neuroinflammatory response to neurodegenerative processes. ${ }^{24} \mathrm{Ng}$ has been identified as a marker of synaptic loss in the early stages of $\mathrm{AD}$, and thereby also affecting cognitive decline..$^{20,25}$ As these processes could be consequences of $A \beta$ aggregation or parallel mechanisms occurring which could mediate the relationship with cognitive decline, it is crucial to increase knowledge about their involvement in AD.

\section{Vascular pathology and biomarkers}

After $\mathrm{AD}$, the second most common form of dementia is vascular dementia (VaD). ${ }^{26}$ Through magnetic resonance imaging (MRI) of the brain white matter hyperintensities $(\mathrm{WMH})$, lacunar infarcts and cerebral microbleeds, all vascular markers and suggested causes of $\mathrm{VaD},{ }^{27}$ can be identified. Similar to $\mathrm{AD}$ pathology, vascular abnormalities have also been associated with cognitive decline ${ }^{28}$ and neuronal injury. ${ }^{29,30}$ Even though these pathologies often co-occur in elderly, ${ }^{31,32}$ it remains uncertain whether memory-clinic patients with both $\mathrm{AD}$ and vascular pathology have more extensive neuronal damage and an increased rate of cognitive decline. Insight into the interrelationship between AD and vascular pathology will be valuable to increase prognostic accuracy for memory-clinic patients and it may provide clues for treatment strategies. 


\section{Risk factors for dementia}

After AD, the second Numerous genetic and environmental factors have been associated with an increased risk of developing dementia. While some are AD specific, like carriership of the apolipoprotein E (APOE) $\varepsilon 4$ allele, ${ }^{33,34}$ others, like increasing age, ${ }^{35}$ are generic risk factors for dementia. In addition, also hypertension, ${ }^{36,37}$ hypercholesterolemia, ${ }^{36-38}$ obesity, ${ }^{39,40}$ depression, ${ }^{41}$ stroke, ${ }^{42}$ smoking, ${ }^{43}$ alcohol use,${ }^{44,45}$ diabetes ${ }^{37}$ and cardiovascular disease ${ }^{37,46}$ have all been associated with an increased risk of cognitive impairment and with both $\mathrm{AD}$-type dementia and $\mathrm{VaD}$. Yet the relationships between these so-called vascular risk factors and the development of AD pathology throughout the course of the disease, are still largely unknown. A better understanding of these relations could help to make primary and secondary prevention strategies more targeted and thereby more beneficial.

\section{Biological correlates of cognition}

Both $\mathrm{AD}^{19,47-49}$ and vascular pathology $\mathrm{y}^{28,50,51}$ have been associated with cognitive impairment and decline long before the dementia phase. Cognitive performance is therefore an important indicator for clinical staging of the disease, which can be assessed via a neuropsychological examination. ${ }^{52}$ By comparing neuropsychological performance to a cognitively normal reference group of the same age, gender and educational level (i.e. normative data), a cognitive impairment can be indentified. ${ }^{53}$ However, this normative data could be biased, as pathological changes related to $\mathrm{AD}$ could already be present in aged cognitively normal individuals, ${ }^{7}$ and could negatively influence cognition. ${ }^{47,54,55}$ Therefore, normative data based on individuals without AD pathology, could improve early detection and predictive accuracy of dementia which may be helpful in memory clinic settings as well as for clinical trials and future research.

\section{Reusing and connecting}

In order to increase and expand knowledge and to thereby overcome current challenges in $\mathrm{AD}$ research, large scaled databases are required to conduct powerful and generalizable analyses. An inexpensive and efficient method of obtaining large datasets which also limits the burden for patients, is reusing existing data and samples. The European Medical Information Framework for AD (EMIF-AD) project was initiated to facilitate the reuse and aggregation of healthcare data and thereby enhance $\mathrm{AD}$ research. 


\section{THESIS AIMS \& OUTLINE}

The general aim of this thesis is to increase knowledge about $\mathrm{AD}$ pathophysiology and improve prognostic accuracy by investigating several biological pathways which could influence the development and course of AD. In the first part of this thesis (Chapter $2 \& 3$ ) we discuss the methodological basis of this thesis: the EMIF-AD project and the characteristics of the EMIF-AD Multimodal Biomarker Discovery (EMIF-AD MBD) study. In the subsequent chapters, we use this methodological basis to addresses the following research questions, based on the theoretical framework shown in Figure 1.

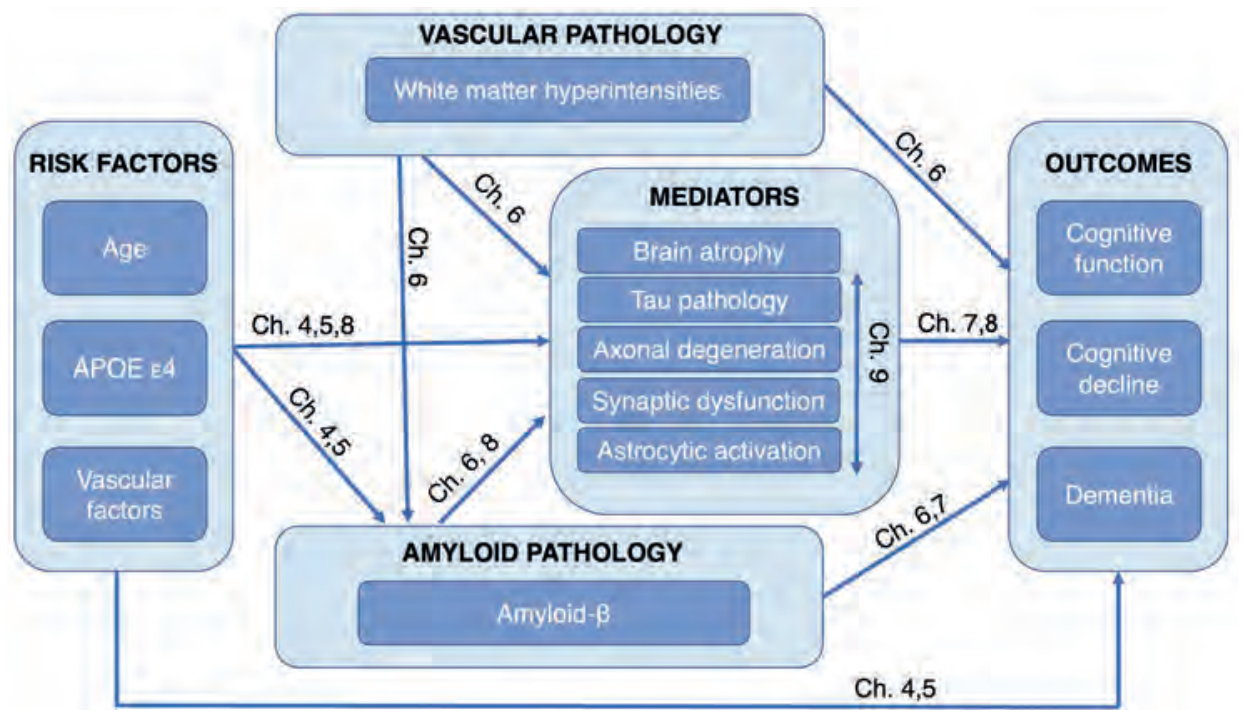

Figure 1: Theoretical framework and outline of this thesis

Are vascular risk factors associated with $A D$ pathology in the pre-dementia stages and do they influence the rate of cognitive decline?

In Chapter 4, we described a study investigating the frequency and influence of dementia risk factors on $\mathrm{AD}$ pathology in individuals with MCI. Individuals were classified by their biomarker profile, based on amyloid and neurodegeneration markers, into four groups. We compared occurrence of risk factors between biomarker groups and investigated the influence of risk factors on rate of cognitive decline. For this study, we used a collated dataset which contains data from 14 prospective cohort studies. In Chapter 5 we examined the frequency and influence of risk factors on AD pathology in cognitively normal individuals, using data from the longitudinal studies of memory and aging at the Knight Alzheimer's Disease Research Center (ADRC) of the Washington University School of Medicine in St. Louis. In addition, we examined a possible causal relationship between 
risk factors and $\mathrm{AD}$ biomarkers by investigating the influence of risk factors on change in CSF biomarker values over time.

Do vascular and $A \beta$ pathology independently contribute to neuronal injury and cognitive decline in memory-clinic patients?

In Chapter 6 we aimed to examine the interrelationship between AD and CVD biomarkers in a non-demented memory-clinic population. We investigated how $\mathrm{A} \beta$ and vascular pathology influenced neuronal injury markers, cognitive decline and progression to dementia. For this study, we used an aggregated European dataset with data from two prospective multicenter cohort studies and one single center cohort.

Can we improve prognostic accuracy of dementia in memory-clinic patients by using normative data for cognitive test based on individuals without AD pathology?

In Chapter 7, we investigated the independent influence of $A \beta$ and tau pathology on cognitive performance in an aggregated test cohort with cognitively normal individuals from eight European studies and the Alzheimer's Disease Neuroimaging Initiative. Subsequently, we examined whether novel normative data based on individuals without AD pathology from the test cohort, would be more accurate in predicting progression to dementia in memory-clinic patients from the independent Maastricht ACL cohort.

Are axonal degeneration, synaptic dysfunction and astrocytic activation related to $A \beta$ in all cognitive stages? And how are these mechanisms associated with APOE genotype and cognition?

Chapter 8 describes results of the EMIF-AD MBD study (Chapter 3), in which we investigated CSF concentrations of NFL, Ng and YKL-40 - markers of axonal degeneration, synaptic dysfunction and neuroinflammation - in individuals across the AD spectrum. We examined the relations of these biomarkers with $\mathrm{A} \beta, A P O E$ genotype and cognitive performance and decline.

Is the biomarker-based subtype SNAP an atypical form of AD and what pathophysiological mechanisms are specifically involved in this subgroup?

In Chapter 9 we aimed to characterize individuals with MCI and an atypical AD biomarker profile: SNAP. In an aggregated dataset with data from 8 European prospective cohorts, we studied CSF concentrations of three A $\beta$ isoforms, NFL, Ng and YKL- 40 . Data-driven clustering analyses were performed to reveal possible subgroups of MCISNAP based on CSF measures. 
The last part of this thesis, Chapter 10, is the general discussion where all findings are summarized and implications for future research, clinical trials and clinical practice are discussed. 


\section{References}

1. Vemuri P, Knopman DS. The role of cerebrovascular disease when there is concomitant Alzheimer disease. Biochimica et biophysica acta. 2016;1862(5):952-956.

2. Lo RY, Jagust WJ, Alzheimer's Disease Neuroimaging I. Vascular burden and Alzheimer disease pathologic progression. Neurology. 2012;79(13):1349-1355.

3. Kalaria RN, Ballard C. Overlap between pathology of Alzheimer disease and vascular dementia. Alzheimer disease and associated disorders. 1999;13 Suppl 3:S115-123.

4. Selkoe DJ. Alzheimer's disease is a synaptic failure. Science. 2002;298(5594):789-791.

5. Raff MC, Whitmore AV, Finn JT. Axonal self-destruction and neurodegeneration. Science. 2002;296(5569):868-871.

6. Heneka MT, Carson MJ, El Khoury J, et al. Neuroinflammation in Alzheimer's disease. The Lancet Neurology. 2015;14(4):388-405.

7. Dubois B, Hampel H, Feldman HH, et al. Preclinical Alzheimer's disease: Definition, natural history, and diagnostic criteria. Alzheimer's \& dementia : the journal of the Alzheimer's Association. 2016;12(3):292-323.

8. Albert MS, DeKosky ST, Dickson D, et al. The diagnosis of mild cognitive impairment due to Alzheimer's disease: recommendations from the National Institute on Aging-Alzheimer's Association workgroups on diagnostic guidelines for Alzheimer's disease. Alzheimer's \& dementia : the journal of the Alzheimer's Association. 2011;7(3):270-279.

9. DeCarli C. Mild cognitive impairment: prevalence, prognosis, aetiology, and treatment. The Lancet Neurology. 2003;2(1):15-21.

10. APA. Diagnostic and Statistical Manual of Mental Disorders: DSM-IV. Washington, DC: American Psychiatric Association; 1994.

11. Jansen WJ, Ossenkoppele R, Knol DL, et al. Prevalence of cerebral amyloid pathology in persons without dementia: a meta-analysis. Jama. 2015;313(19):1924-1938.

12. Bateman RJ, Xiong C, Benzinger TL, et al. Clinical and biomarker changes in dominantly inherited Alzheimer's disease. The New England journal of medicine. 2012;367(9):795-804.

13. Blennow K, Zetterberg H. Cerebrospinal fluid biomarkers for Alzheimer's disease. Journal of Alzheimer's disease : JAD. 2009;18(2):413-417.

14. Mayeux R. Early Alzheimer's disease. New England Journal of Medicine. 2010;362(23):2194-2201.

15. Price JL, Morris JC. Tangles and plaques in nondemented aging and "preclinical" Alzheimer's disease. Annals of neurology. 1999;45(3):358-368.

16. Hardy JA, Higgins GA. Alzheimer's disease: the amyloid cascade hypothesis. Science. 1992;256(5054):184-185.

17. Jack CR, Knopman DS, Jagust WJ, et al. Tracking pathophysiological processes in Alzheimer's disease: an updated hypothetical model of dynamic biomarkers. The Lancet Neurology. 2013;12(2):207-216.

18. Chételat G, Villemagne VL, Bourgeat $P$, et al. Relationship between atrophy and $\beta$-amyloid deposition in Alzheimer disease. Annals of neurology. 2010;67(3):317-324.

19. Vos SJ, Verhey F, Frolich L, et al. Prevalence and prognosis of Alzheimer's disease at the mild cognitive impairment stage. Brain : a journal of neurology. 2015;138(Pt 5):1327-1338.

20. Kester MI, Teunissen CE, Crimmins DL, et al. Neurogranin as a Cerebrospinal Fluid Biomarker for Synaptic Loss in Symptomatic Alzheimer Disease. JAMA Neurol. 2015;72(11):1275-1280.

21. Zetterberg H, Skillback T, Mattsson N, et al. Association of Cerebrospinal Fluid Neurofilament Light Concentration With Alzheimer Disease Progression. JAMA Neurol. 2016;73(1):60-67.

22. Janelidze S, Hertze J, Zetterberg H, et al. Cerebrospinal fluid neurogranin and YKL-40 as biomarkers of Alzheimer's disease. Ann Clin Transl Neurol. 2016;3(1):12-20.

23. Bruno D, Pomara N, Nierenberg J, et al. Levels of cerebrospinal fluid neurofilament light protein in healthy elderly vary as a function of TOMM40 variants. Experimental gerontology. 2012;47(5):347-352. 
24. Antonell A, Mansilla A, Rami L, et al. Cerebrospinal fluid level of YKL-40 protein in preclinical and prodromal Alzheimer's disease. Journal of Alzheimer's disease : JAD. 2014;42(3):901-908.

25. Portelius E, Zetterberg H, Skillback T, et al. Cerebrospinal fluid neurogranin: relation to cognition and neurodegeneration in Alzheimer's disease. Brain : a journal of neurology. 2015;138(Pt 11):3373-3385.

26. Jorm AF, Jolley D. The incidence of dementia: a meta-analysis. Neurology. 1998;51(3):728-733.

27. Kurz A, Riemenschneider M, Wallin A. Potential biological markers for cerebrovascular disease. International psychogeriatrics / IPA. 2003;15 Suppl 1:89-97.

28. Jellinger KA. Understanding the pathology of vascular cognitive impairment. Journal of the neurological sciences. 2005;229-230:57-63.

29. Hesse C, Rosengren L, Andreasen N, et al. Transient increase in total tau but not phospho-tau in human cerebrospinal fluid after acute stroke. Neuroscience letters. 2001;297(3):187-190.

30. Bastos-Leite AJ, van der Flier WM, van Straaten EC, Staekenborg SS, Scheltens P, Barkhof F. The contribution of medial temporal lobe atrophy and vascular pathology to cognitive impairment in vascular dementia. Stroke; a journal of cerebral circulation. 2007;38(12):3182-3185.

31. Attems J, Jellinger KA. The overlap between vascular disease and Alzheimer's disease--lessons from pathology. BMC Med. 2014;12:206.

32. Schneider JA, Aggarwal NT, Barnes L, Boyle P, Bennett DA. The neuropathology of older persons with and without dementia from community versus clinic cohorts. Journal of Alzheimer's disease : JAD. 2009;18(3):691-701.

33. $\mathrm{Bu}$ G. Apolipoprotein E and its receptors in Alzheimer's disease: pathways, pathogenesis and therapy. Nat Rev Neurosci. 2009;10(5):333-344.

34. Strittmatter WJ, Roses AD. Apolipoprotein E and Alzheimer disease. Proceedings of the National Academy of Sciences of the United States of America. 1995;92(11):4725-4727.

35. Matthews F, Brayne C, Medical Research Council Cognitive F, Ageing Study I. The incidence of dementia in England and Wales: findings from the five identical sites of the MRC CFA Study. PLoS Med. 2005;2(8):e193.

36. de Bruijn RF, Ikram MA. Cardiovascular risk factors and future risk of Alzheimer's disease. BMC Med. 2014;12:130.

37. Duron E, Hanon O. Vascular risk factors, cognitive decline, and dementia. Vasc Health Risk Manag. 2008;4(2):363-381.

38. Helzner EP, Luchsinger JA, Scarmeas N, et al. Contribution of vascular risk factors to the progression in Alzheimer disease. Archives of neurology. 2009;66(3):343-348.

39. Anstey KJ, Cherbuin N, Budge M, Young J. Body mass index in midlife and late-life as a risk factor for dementia: a meta-analysis of prospective studies. Obes Rev. 2011;12(5):e426-437.

40. Kivipelto M, Ngandu T, Fratiglioni L, et al. Obesity and vascular risk factors at midlife and the risk of dementia and Alzheimer disease. Archives of neurology. 2005;62(10):1556-1560.

41. Ownby RL, Crocco E, Acevedo A, John V, Loewenstein D. Depression and risk for Alzheimer disease: systematic review, meta-analysis, and metaregression analysis. Archives of general psychiatry. 2006;63(5):530-538.

42. Honig LS, Tang M-X, Albert S, et al. Stroke and the risk of Alzheimer disease. Archives of neurology. 2003;60(12):1707-1712.

43. Anstey KJ, von Sanden C, Salim A, O'Kearney R. Smoking as a risk factor for dementia and cognitive decline: a meta-analysis of prospective studies. American journal of epidemiology. 2007;166(4):367-378.

44. Anstey KJ, Mack HA, Cherbuin N. Alcohol consumption as a risk factor for dementia and cognitive decline: meta-analysis of prospective studies. Am J Geriatr Psychiatry. 2009;17(7):542-555.

45. Jauhar S, Marshall EJ, Smith ID. Alcohol and cognitive impairment. Advances in psychiatric treatment. 2014;20(5):304-313.

46. Hasnain M, Vieweg WV. Possible role of vascular risk factors in Alzheimer's disease and vascular dementia. Current pharmaceutical design. 2014;20(38):6007-6013. 


\section{Chapter 1}

47. Jansen WJ, Ossenkoppele R, Tijms BM, et al. Association of Cerebral Amyloid-beta Aggregation With Cognitive Functioning in Persons Without Dementia. JAMA Psychiatry. 2017.

48. Baker JE, Lim YY, Pietrzak RH, et al. Cognitive impairment and decline in cognitively normal older adults with high amyloid- $\beta$ : A meta-analysis. Alzheimer's \& Dementia: Diagnosis, Assessment \& Disease Monitoring. 2017.

49. Petersen RC, Wiste HJ, Weigand SD, et al. Association of elevated amyloid levels with cognition and biomarkers in cognitively normal people from the community. JAMA neurology. 2016;73(1).

50. Gorelick PB, Scuteri A, Black SE, et al. Vascular contributions to cognitive impairment and dementia. Stroke; a journal of cerebral circulation. 2011;42(9):2672-2713.

51. Prins ND, Scheltens P. White matter hyperintensities, cognitive impairment and dementia: an update. Nat Rev Neurol. 2015;11(3):157-165.

52. Lezak MD, Howieson DB, Loring DW. Neuropsychological Assessment. Oxford, UK: Oxford University Press 2004.

53. Petersen RC, Smith GE, Waring SC, Ivnik RJ, Tangalos EG, Kokmen E. Mild cognitive impairment: clinical characterization and outcome. Archives of neurology. 1999;56(3):303-308.

54. Mormino EC, Betensky RA, Hedden T, et al. Synergistic effect of beta-amyloid and neurodegeneration on cognitive decline in clinically normal individuals. JAMA Neurol. 2014;71(11):1379-1385.

55. Vos SJ, Xiong C, Visser PJ, et al. Preclinical Alzheimer's disease and its outcome: a longitudinal cohort study. The Lancet Neurology. 2013;12(10):957-965. 




\section{Part}

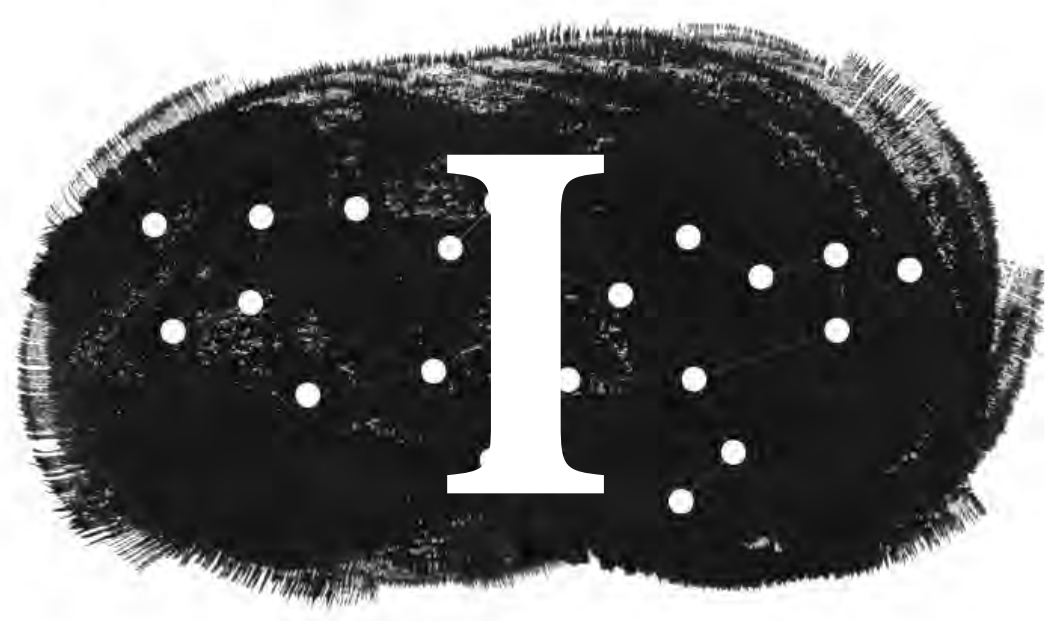

European Medical Information Framework for Alzheimer's disease (EMIF-AD) 


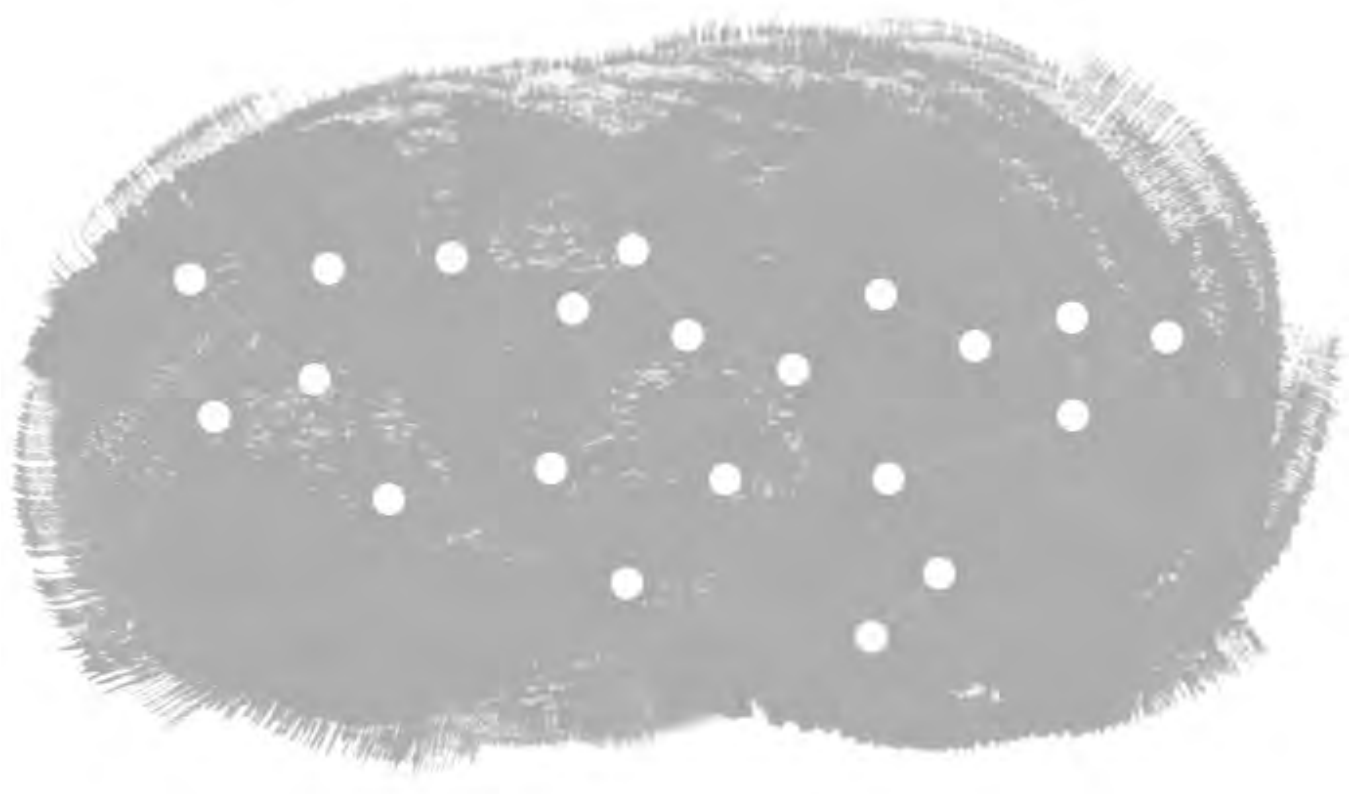




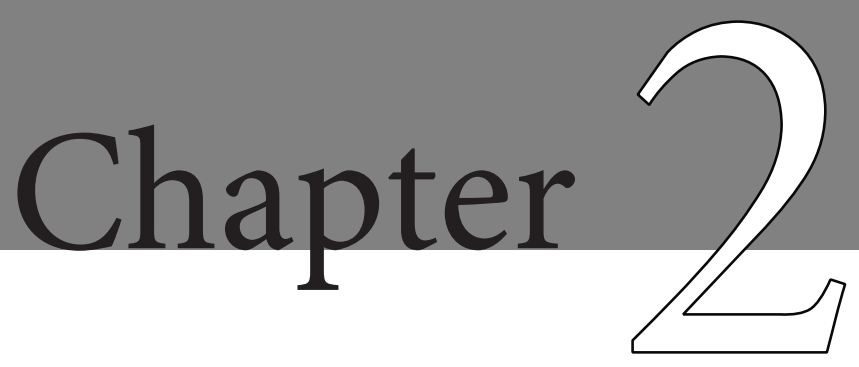

European Medical Information Framework for Alzheimer's disease (EMIF-AD):

An infrastructure to reuse and combine existing data

Isabelle Bos, Stephanie J.B. Vos, José Luis Oliveira, Rudi Verbeeck, Janneke Schoots - van der Ploeg, Michel Van Speybroeck, Nigel Hughes, Bart Vannieuwenhuysen, Johannes Streffer, Simon Lovestone, Pieter Jelle Visser 


\section{ABSTRACT}

Background: Large-scaled studies are needed to enhance Alzheimer's disease (AD) research. The European Medical Information Framework for AD (EMIF-AD) project was initiated to facilitate the process of reusing and combining existing AD-related data. This report describes the data-sharing infrastructure that has been developed in EMIF-AD.

Methods: We developed several facilities to make data findable and accessible for researchers. The EMIF-AD Catalogue is a public, online repository which provides detailed meta-data on AD-related studies and allows researchers to search and identify studies for future collaborations. The online data platform is a secure environment, where data can be stored, harmonized, pooled and analyzed. The AD-Switchbox allows for pooled analyses on local databases.

Results: Currently, meta-data about 53 studies is uploaded in the EMIF-AD Catalogue, which has more than 400 users worldwide. The data platform contains subject-level data from 14 studies with data on 3394 participants. The developments regarding the ADSwitchbox are ongoing.

Conclusion: The EMIF-AD infrastructure has facilitated numerous research collaborations and is currently being used in other major research projects. In the future, we intent to connect to other data sharing initiatives to create an integrated portfolio of $\mathrm{AD}$ data sharing facilities worldwide. 


\section{INTRODUCTION}

Alzheimer's disease (AD) is the most common form of dementia, ${ }^{1,2}$ for which currently no effective treatment exists. ${ }^{3,4}$ Many scientific initiatives are ongoing to understand the underlying mechanisms of $\mathrm{AD}$, discover biomarkers and to develop disease-modifying treatments. However, to overcome current challenges in $\mathrm{AD}$ research powerful and generalizable analyses should be conducted, which require large scaled databases. Such voluminous datasets can be generated by reusing and combining existing data.

The European Medical Information Framework for Alzheimer's disease (EMIF-AD, http://www.emif.eu) has been initiated to serve as a platform to facilitate combining and reusing $\mathrm{AD}$-related data. Within EMIF-AD a catalogue has been developed containing meta-data on existing AD cohorts, which allows identification of studies suitable for novel collaborations. In addition, an infrastructure for data sharing, combining and storing was arranged using the tranSMART platform. Lastly, also a 'AD-Switchbox' is currently being development which aims to connect local databases to each other. Through these facilities, EMIF-AD aims to identify novel diagnostic and prognostic markers for the preclinical and prodromal stages of $\mathrm{AD}$ within existing, aggregated data.

EMIF-AD receives support from the Innovative Medicines Initiative Joint Undertaking (IMI-JU) and is a collaboration between European Federations of Pharmaceutical Industries and Associations' (EFPIA) and more than twenty academic partners. EMIF-AD is part of the overall EMIF project, which aims also encompasses the EMIF-Metabolic and EMIF-platform communities. EMIF-Metabolic focuses on the identification of diagnostic markers and therapeutic targets for metabolic diseases, ${ }^{5}$ and EMIF-platform provides technological expertise in building data sharing platforms, accessing data from electronic health registries and develops an ethical and legal framework for data sharing. ${ }^{6}$

In this report, we will describe the different data-sharing infrastructures that have been developed within EMIF-AD, explain the procedures for sharing and requesting data, and discuss similar data sharing initiatives and future goals.

\section{CATALOGUE}

The EMIF-AD Catalogue (http://bioinformatics.ua.pt/emif) is an online repository of meta-data on AD-related studies. It provides detailed information on study characteristics, data collection and study protocols of 53 AD-related studies, listed in Table 1 . The Catalogue enables researchers to search, compare and identify studies of interest for their specific research question. For example, a search can be conducted to identify studies that collected blood samples and magnetic resonance imaging (MRI) scans in patients with 
mild cognitive impairment (MCI), but it is not possible to search for blood values or imaging results of individual subjects. Below we will elaborate on the characteristics and facilities of the EMIF-AD Catalogue.

Table 1: AD studies connected to the EMIF-AD infrastructure in March 2018

\begin{tabular}{|c|c|c|c|c|}
\hline Study acronyms & Study name & Country & $\begin{array}{l}\text { Meta-data in } \\
\text { Catalogue }\end{array}$ & $\begin{array}{l}\text { Subject-level } \\
\text { data on } \\
\text { platform }{ }^{*}\end{array}$ \\
\hline Actifcare & Acces to timely formal care & European MC & $\mathrm{x}$ & \\
\hline $\mathrm{ADC}$ & Amsterdam Dementia Cohort & The Netherlands & $\mathrm{x}$ & $\mathrm{x}$ \\
\hline Addneuromed & Addneuromed & European MC & $\mathrm{x}$ & $\mathrm{x}$ \\
\hline ADGEN & Kuopio-ADGEN & Finland & $\mathrm{x}$ & \\
\hline ADNI-1 & AD Neuroimaging Initiative - 1 & US MC & $\mathrm{x}$ & \\
\hline ADNI-2 & AD Neuroimaging Initiative - 2 & US MC & $\mathrm{x}$ & \\
\hline ADNI-GO & AD Neuroimaging Initiative - GO & US MC & $\mathrm{x}$ & \\
\hline AgeCoDe & $\begin{array}{l}\text { Ageing, Cognition and Dementia in } \\
\text { Primary care }\end{array}$ & Germany & $\mathrm{x}$ & \\
\hline Antwerp cohort & Antwerp cohort & Belgium & $\mathrm{x}$ & $\mathrm{x}$ \\
\hline ARWIBO & AD Repository Without Borders & Italy & $\mathrm{x}$ & \\
\hline Athens cohort & Athens cohorts & Greece & $\mathrm{x}$ & \\
\hline BCN - Sant Pau & Barcelona Sant Pau & Spain & $\mathrm{x}$ & $\mathrm{x}$ \\
\hline CAIDE & $\begin{array}{l}\text { Cardiovascular Risk Factors, Aging } \\
\text { and Dementia }\end{array}$ & Finland & $\mathrm{x}$ & \\
\hline CogLaus & Lausanne cohort & Switzerland & $\mathrm{x}$ & $\mathrm{x}$ \\
\hline $\mathrm{DCN}$ & Dementia Competence Network & German Multicenter & & \\
\hline DCR & Dementia Case Register & United Kingdom & $\mathrm{x}$ & \\
\hline $\mathrm{DeNoPa}$ & De Novo Parkinson & Germany & $\mathrm{x}$ & \\
\hline DESCRIPA & $\begin{array}{l}\text { Development of screening guidelines } \\
\text { for predementia } \mathrm{AD}\end{array}$ & European MC & $\mathrm{x}$ & $\mathrm{x}$ \\
\hline $\begin{array}{l}\text { DESCRIPA } \\
\text { population }\end{array}$ & $\begin{array}{l}\text { Development of screening guidelines } \\
\text { for predementia } \mathrm{AD} \text { - population }\end{array}$ & European MC & $\mathrm{x}$ & \\
\hline DiMi & Diagnostic molecular imaging & European MC & $\mathrm{x}$ & \\
\hline Donepezil & Donepezil & France & $\mathrm{x}$ & \\
\hline EADC-PET & European AD Consortium - PET & European MC & $\mathrm{x}$ & \\
\hline EADC prodromal & $\begin{array}{l}\text { European AD Consortium - } \\
\text { Prodromal AD }\end{array}$ & European MC & $\mathrm{x}$ & \\
\hline EDAR & $\begin{array}{l}\text { Beta amyloid oligomers in the early } \\
\text { diagnosis of } \mathrm{AD}\end{array}$ & European MC & $\mathrm{x}$ & $\mathrm{x}$ \\
\hline FACEHBI & $\begin{array}{l}\text { Fundació ACE Healthy Brain } \\
\text { Initiative }\end{array}$ & Spain & $\mathrm{x}$ & \\
\hline FINGER & Finnish Geriatric Intervention study & Finland & $\mathrm{x}$ & \\
\hline GAP & Gipuzkoa Alzheimer Proyecto & Spain & $\mathrm{x}$ & $\mathrm{x}$ \\
\hline Genoa & Genoa cohort & Italy & $\mathrm{x}$ & $\mathrm{x}$ \\
\hline Gothenburg MCI & $\begin{array}{l}\text { Gothenburg mild cognitive } \\
\text { impairment }\end{array}$ & Sweden & $\mathrm{x}$ & $\mathrm{x}$ \\
\hline $\mathrm{H} 70$ & Gothenburg population studies & Sweden & $\mathrm{x}$ & \\
\hline
\end{tabular}




\begin{tabular}{|c|c|c|c|c|}
\hline Study acronyms & Study name & Country & $\begin{array}{l}\text { Meta-data in } \\
\text { Catalogue }\end{array}$ & $\begin{array}{l}\text { Subject-level } \\
\text { data on } \\
\text { platform* }\end{array}$ \\
\hline HELIAD & \multicolumn{2}{|c|}{$\begin{array}{l}\text { Hellenic Longitudinal Investigation of Greece } \\
\text { Aging and Diet }\end{array}$} & $\mathrm{x}$ & \\
\hline IDIBAPS & $\begin{array}{l}\text { Instiut d'investigacions Biomèdiques } \\
\text { August Pi I Sunyer }\end{array}$ & Spain & $\mathrm{x}$ & $\mathrm{x}$ \\
\hline ILSA & Italian Longitudinal Study on Aging & Italy & $\mathrm{x}$ & \\
\hline $\mathrm{IMAP}+$ & $\begin{array}{l}\text { Multimodality Imaging of Early-stage } \\
\text { AD }\end{array}$ & France & $\mathrm{x}$ & $\mathrm{x}$ \\
\hline IPREA & \multicolumn{2}{|c|}{$\begin{array}{l}\text { Italian Project on the epidemiology of Italy } \\
\text { AD }\end{array}$} & $\mathrm{x}$ & \\
\hline LASA & \multicolumn{2}{|c|}{ Longitudinal Aging study Amsterdam The Netherlands } & $\mathrm{x}$ & \\
\hline LeARN & $\begin{array}{l}\text { Leiden Alzheimer Research } \\
\text { Nederland }\end{array}$ & Dutch Multicenter & $\mathrm{x}$ & \\
\hline Leuven & Leuven cohort & Belgium & $\mathrm{x}$ & $\mathrm{x}$ \\
\hline L-MCI & Kuopio Longitudinal MCI study & Finland & $\mathrm{x}$ & \\
\hline MCI-GO & MCI - Gothenborg Oslo project & Norway & $\mathrm{x}$ & \\
\hline Memento & Memento cohort & France & $\mathrm{x}$ & \\
\hline Milan cohort & Milan cohort & Italy & $\mathrm{x}$ & \\
\hline MRC-CFAS & $\begin{array}{l}\text { Medical Research Council Cognitive } \\
\text { Function on Ageing study }\end{array}$ & United Kingdom & $\mathrm{x}$ & \\
\hline NEST-DD & $\begin{array}{l}\text { Network for Efficiency and } \\
\text { Standardization of dementia diagnosis }\end{array}$ & $\begin{array}{l}\text { European } \\
\text { sMulticenter }\end{array}$ & $\mathrm{x}$ & \\
\hline Oslo & Oslo cohort & Norway & $\mathrm{x}$ & \\
\hline Perugia & Perugia cohort & Italy & $\mathrm{x}$ & \\
\hline Pharmacog & PharmaCog & $\begin{array}{l}\text { European } \\
\text { Multicenter }\end{array}$ & $\mathrm{x}$ & $\mathrm{x}$ \\
\hline PPSW & $\begin{array}{l}\text { Prospect population study of women } \\
\text { in Gothenburg }\end{array}$ & Sweden & $\mathrm{x}$ & \\
\hline Pre-Al study & Prediction of $\mathrm{AD}$ & France & $\mathrm{x}$ & \\
\hline PSI & Parelsnoer institute & Dutch Multicenter & $\mathrm{x}$ & \\
\hline Recall - HNR & Heinz Nixdorf Recall study & Germany & $\mathrm{x}$ & \\
\hline ReGAl Project & Rete Geriatrica Alzheimer & Italy & $\mathrm{x}$ & \\
\hline SNAC-K & $\begin{array}{l}\text { Swedish National Study on Aging and } \\
\text { Care in Kungsholmen }\end{array}$ & Sweden & $\mathrm{x}$ & \\
\hline
\end{tabular}

${ }^{\star}$ Whole dataset or subset of variables and/or participants. Abbreviations: AD = Alzheimer's Disease; $\mathrm{MC}=\mathrm{mul}-$ ticenter

\section{Information categories in the EMIF-AD Catalogue}

The EMIF-AD Catalogue has 27 categories with information on study characteristics, data sharing preferences and type of data collected (Table 2). For instance, the category 'Neuropsychiatric scales' lists whether a study administered neuropsychiatric scales and, if so, which rating scales were used and whether they were administered repeatedly. Another example is the category 'MRI' which contains, among other things, information on the type of 
scanner and the acquisition parameters. A study contact person is responsible for entering the information online and can amend or update the information at any given moment.

Table 2: Information categories in the EMIF-AD Catalogue

\begin{tabular}{ll}
\hline 1. Database general information & 15. Cognitive screening tests \\
2. Key publications & 16. Neuropsychological tests \\
3. Data access & 17. Physical Examination \\
4. Study characteristics & 18. Lifestyle Factors \\
5. Inclusion/exclusion criteria & 19. Blood collection \\
6. Number of subjects & 20. CSF collection \\
7. Clinical information & 21. Urine collection \\
8. Dementia and functional rating scales & 22. MRI \\
9. Subjective cognitive impairment & 23. PET \\
10. Neuropsychiatric scales & 24. CT \\
11. Quality of life scales & 25. SPECT \\
12. Caregiver scales & 26. Electrophysiology \\
13. Health Resource Utilization & 27. Neuropathology \\
14. Remote Monitoring Technologies &
\end{tabular}

Abbreviations: $\mathrm{CSF}=$ cerebrospinal fluid, CT = computer tomography, $\mathrm{MRI}=$ Magnetic Resonance Imaging, PET $=$ positron emission tomography, SPECT $=$ single-photon emission computed tomography

\section{Catalogue search}

The EMIF-AD Catalogue offers several search functionalities. A keyword search option was developed to quickly find studies of interest using only one search term. The advanced search option allows to create more complex queries, combining several search criteria using Boolean terms. Figure 1 shows an advanced search query searching for studies that collected plasma samples and MRI scans in individuals with MCI or AD-type dementia.

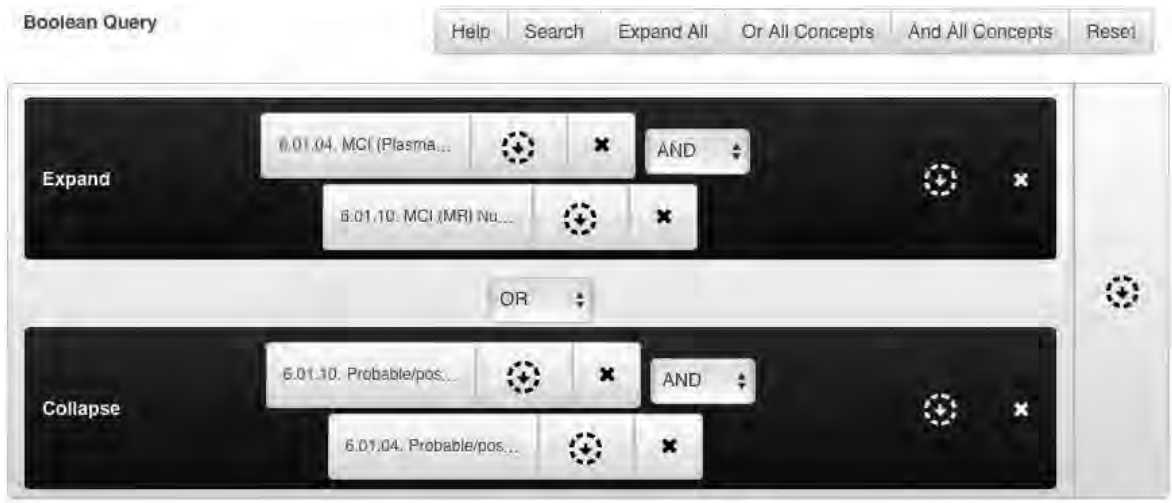

Figure 1. EMIF-AD Catalogue advanced search query

Query searching for studies that collected plasma samples and MRI scans in individuals with MCI or AD-type dementia 


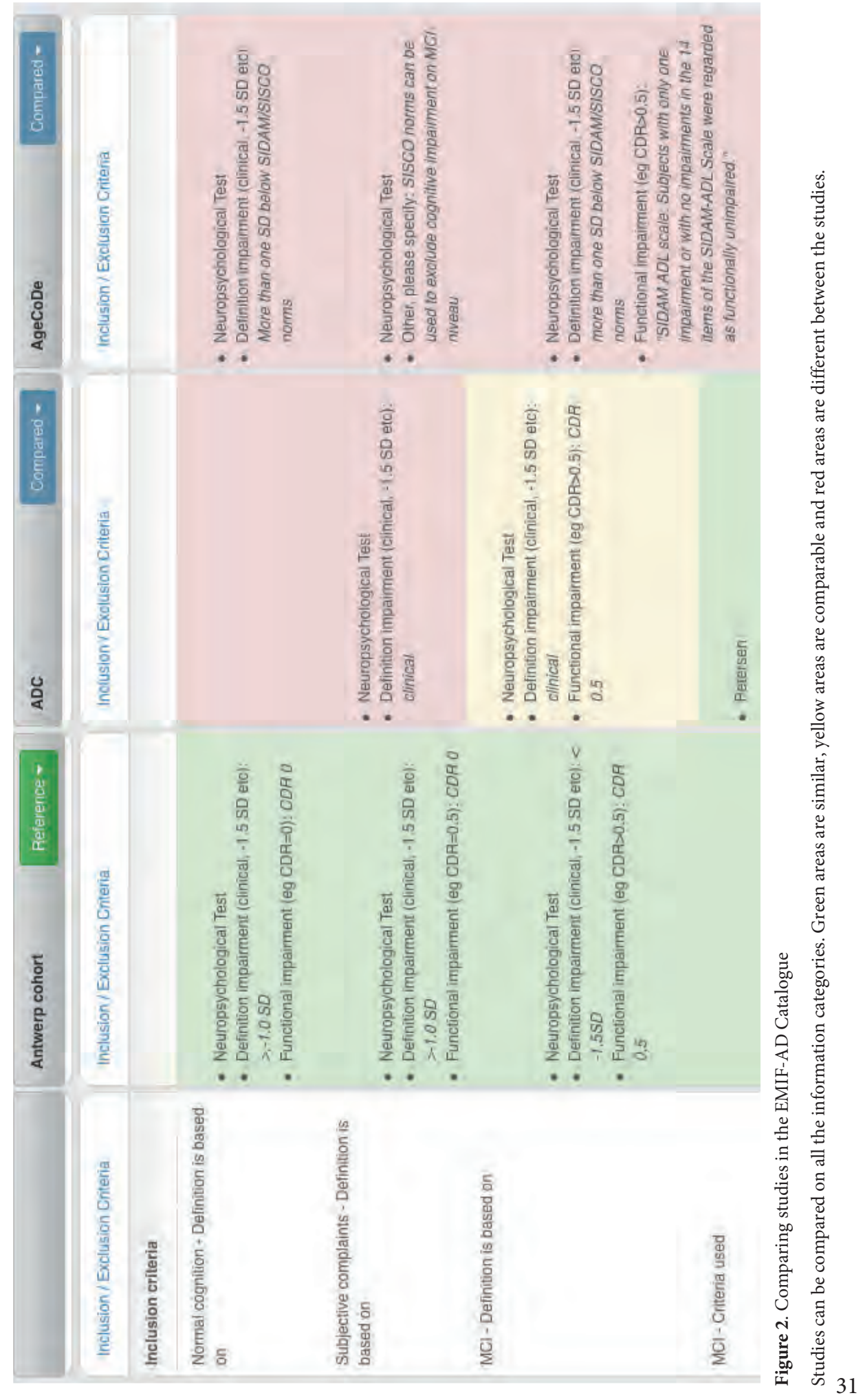


Both the keyword and advanced search produce a list with studies that meet the applied search criteria. In addition, studies can be compared using the Compare option. This provides an overview of the overlap and differences among up to three studies in all the information categories (Figure 2). All information from the EMIF-AD Catalogue can also be exported to view and browse offline.

\section{Catalogue statistics}

So far, the EMIF-AD Catalogue has been successfully used to identify studies for numerous collaborative projects ${ }^{7-11}$. Currently, 413 researchers from 32 countries around the world have registered. In 2017, the EMIF-AD Catalogue had an average of 204 views per month, and 164 Advanced Searches were conducted last year. Besides the EMIF-AD Catalogue, which specifically focusses on AD cohorts, EMIF has also developed a catalogue for European Electronic Healthcare Registers (EHR) and has supported the development of catalogues for other European projects such as the IMI-project European Prevention of Alzheimer's Dementia Consortium (EPAD) ${ }^{12}$ and the Dementias platform UK (DPUK, https://www.dementiasplatform.uk).

\section{DATA PLATFORM}

In order to store, share and combine subject level data, a data platform was developed using the open-source tranSMART system (http://emif.custodix.com/transmart). This data platform stores harmonized data, in an organized and secure manner. On the EMIFAD data platform subject-level data are only visible and finable for researchers whom have been granted access by the data-owners. The data platform facilitates the process of combining data from different studies for pooled online or offline analyses. Prior to upload, the data is validated and anonymized by the data-owner.

\section{Harmonizing and combining data}

The process of harmonizing and combining data requires several steps. First, data is recoded into common standards, which requires the recoding of variable names and values. In this process, the standards according to the Clinical Data Interchange Standards Consortium (CDISC, http://www.cdisc.org) serve as a guideline. Next, to combine variables that measure the same construct but use different instruments across studies, we apply two harmonization approaches. One approach is to express values as standardized values (Z-scores) based on local normative data. This approach can, for example, be used to pool neuropsychological data which measure the same construct (e.g. delayed recall on a memory task) with different tests. A second method is to dichotomize variables as normal 
or abnormal, using local cut-offs. This method can for instance be used when comparing analytes in bio fluids that have been analyzed using different assays. In order to facilitate recoding and harmonization a 'knowledge object library' has been developed. The knowledge object library consists of scripts to recode a wide range of variables into common variables and values, and to create standardized or dichotomized values. After recoding and harmonizing, data is uploaded to the data platform for each cohort separately. On the data platform, data can be combined across cohorts for specific analyses. Data can be analyzed online using build-in facilities, or can be exported for offline analyses. Data access settings are fully controlled by the data-owner.

Table 3. Data categories on EMIF-AD data platform

\begin{tabular}{|c|c|}
\hline Category & Description \\
\hline Clinical Information & $\begin{array}{l}\text { Data on comorbidities, diagnoses, family history, lifestyle factors, } \\
\text { medication use, physical examination }\end{array}$ \\
\hline Cognitive Screening Tests & $\begin{array}{l}\text { Data from several cognitive screening tests such as the Mini Mental State } \\
\text { Examination or the Montreal Cognitive Assessment }\end{array}$ \\
\hline Demographics & Data on age, country and sex \\
\hline Imaging & Data from MRI and PET imaging techniques \\
\hline Laboratory Test Results & Data from blood or CSF analyses \\
\hline Neuropsychological Examination & $\begin{array}{l}\text { Data from neuropsychological tests from the cognitive domains: attention } \\
\text { and executive functioning, intelligence, language, memory and } \\
\text { visuoconstruction }\end{array}$ \\
\hline Pharmacogenetics Findings & Data from genetic analyses \\
\hline Rating Scales & $\begin{array}{l}\text { Data from rating scale assessments such as neuropsychiatric scales or } \\
\text { quality of life scales }\end{array}$ \\
\hline Subject Characteristics & Data on educational level, employment status and living situation \\
\hline
\end{tabular}

Abbreviations: $\mathrm{CSF}=$ cerebrospinal fluid, $\mathrm{MRI}=$ magnetic resonance imaging, $\mathrm{PET}=$ positron emission tomography.

\section{Data platform statistics}

Currently data from 14 studies is uploaded to the data platform (Table 1). Of these studies, ten are single center studies and four are multicenter studies. In total, the data platform contains data on 3394 participants of which 824 (24\%) were cognitively normal at baseline, 400 (12\%) had subjective cognitive deficits, 1571 (46\%) had mild cognitive impairment, 525 (15\%) had AD-type dementia, 57 (2\%) had another type of dementia and 17 (1\%) had another or no diagnosis at baseline. The average age at baseline of all participants was 70.2 years (SD 8.7).

In total, the data platform has data on more than 300 variables, divided into 9 main categories (Table 3). Approximately 150 variables have been collected repeatedly; at baseline and on available follow-up visits. As not all studies collected similar data, the number of variables 
varies amongst the studies, but all studies have data on at least 40 shared and harmonized variables. This allows for pooling of data across studies in an elaborative set of variables.

\section{AD-SWITCHBOX}

In order to facilitate automatic harmonization and access to subject-level data which is stored in local databases, the 'AD-Switchbox' is being developed in collaboration with the IMI-EPAD project. ${ }^{12}$ The AD-Switchbox uses the ontology from the knowledge object library to allow automated data extraction, data harmonization and data transfer to the EMIF-AD data platform. It is installed locally at the data-owner's institution such that data can be updated automatically. The AD-Switchbox provides a novel approach to update data on the EMIF-AD data platform and to perform pooled analyses of data that are stored in local databases.

\section{USING THE EMIF-AD INFRASTRUCTURE}

After creating an account (http://bioinformatics.ua.pt/emif), researchers can search the EMIF-AD Catalogue for studies that may have data for their research projects. To invite studies for a research collaboration, a Study Request can be submitted to the EMIF-AD Catalogue, which includes a short description of the project. Depending on the participating studies and the research question, the exchange of data can either be done via the EMIF-AD data sharing facilities or independently from EMIF-AD. The subject-level data on the EMIF-AD data platform are accessible upon invite, after consent of each dataowner of the involved studies.

\section{COMPARITIVE OVERVIEW OF OTHER DATA SHARING INITIATIVES}

Besides EMIF-AD there are a few other AD-related data sharing initiatives. In Table 4 we compared the EMIF-AD facilities to those of DPUK, to the Global Alzheimer's Association Interactive Network (GAAIN; https:/www.gaain.org/)13 and the Global Cohort Portal of the EU Joint Programme - Neurodegenerative Disease Research (JPND; http://www.neurodegenerationresearch.eu/jpnd-global-cohort-portal/). DPUK aims to connect population studies predominantly from the UK, containing information regarding dementia, while GAAIN aims to unite divers and geographically distributed AD-re- 
lated data. The JPND Global Portal aims to facilitate information exchange and collaborations to enable greater volume of neurodegenerative disease research. Although there are differences between the facilities that have been created in the context of these data sharing initiatives, they all serve the same purpose of facilitating the reuse and combining of existing data to enhance dementia research. A collaboration between these initiatives would enable the opportunity to benefit from the advantages of each project and thereby overcome current challenges in data sharing.

Table 4. Comparison of AD-related data sharing initiatives

\begin{tabular}{|c|c|c|c|}
\hline \multirow[b]{2}{*}{ Initiatives } & \multicolumn{3}{|l|}{ Data sharing facilities } \\
\hline & Study identifier & Central data pooling and sharing & Connecting local databases \\
\hline EMIF-AD & $\begin{array}{l}\text { - Detailed information with } \\
\text { study characteristics and col- } \\
\text { lection protocols of } 53 \text { AD- } \\
\text { related studies worldwide } \\
\text { - Advanced search, compare } \\
\text { and export facilities } \\
\text { - Supports other projects such } \\
\text { as DPUK and EPAD }\end{array}$ & $\begin{array}{l}\text { - Subject-level data on } 3394 \text { partici- } \\
\text { pants available upon request on } \\
\text { online data platform } \\
\text { - Baseline and follow-up data on } \\
\text { wide variety of variables, with a } \\
\text { set of } \pm 40 \text { shared, harmonized } \\
\text { variables } \\
\text { - Supports pooled online analyses } \\
\text { and exporting of data }\end{array}$ & $\begin{array}{l}\text { - Allows for pooled analyses } \\
\text { of local databases } \\
\text { - Enables searches in subject- } \\
\text { level data in a wide variety } \\
\text { of variables } \\
\text { - Connected to other projects } \\
\text { such as EPAD }\end{array}$ \\
\hline DPUK & $\begin{array}{l}\text { - Originated from EMIF-AD } \\
\text { Catalogue } \\
\text { - Information on } 41 \text { popula- } \\
\text { tion studies in schematic } \\
\text { overview } \\
\text { - Search facilities and details } \\
\text { on collection protocols are } \\
\text { limited }\end{array}$ & $\begin{array}{l}\text { - Subject-level data available upon } \\
\text { request, within workspace envi- } \\
\text { ronment } \\
\text { - Baseline and follow-up data on a } \\
\text { wide variety of variables } \\
\text { - No pooled analyses, nor exporting } \\
\text { facilities or data sharing outside } \\
\text { DPUK }\end{array}$ & $\begin{array}{l}\text { - No facility to connect local } \\
\text { databases }\end{array}$ \\
\hline GAAIN & $\begin{array}{l}\text { - Information on } 34 \text { AD-re- } \\
\text { lated studies worldwide } \\
\text { - Keyword search across stud- } \\
\text { ies or explore single studies } \\
\text { - Build cohort in Cohort Scout } \\
\text { searches } \\
\text { - No details on collection pro- } \\
\text { tocols }\end{array}$ & $\begin{array}{l}\text { - Instant visualization and prelimi- } \\
\text { nary analysis of }>100.000 \text { subjects } \\
\text { using online platform } \\
\text { - Thousands of variables, small har- } \\
\text { t monized set across studies } \\
\text { - Supports pooled preliminary anal- } \\
\text { yses for any harmonized variables }\end{array}$ & $\begin{array}{l}\text { - Pooled analyses of local da- } \\
\text { tabases } \\
\text { - Enables searches in subject- } \\
\text { - level any variable } \\
\text { - Any variables can be used } \\
\text { - for analyses }\end{array}$ \\
\hline $\begin{array}{l}\text { JPND } \\
\text { Global } \\
\text { Cohort } \\
\text { Portal }\end{array}$ & $\begin{array}{l}\text { - Information on } 110 \text { studies } \\
\text { on neurodegenerative dis- } \\
\text { eases } \\
\text { - Allows for specification per } \\
\text { disease and has search facili- } \\
\text { ties } \\
\text { - No details on study materials } \\
\text { or collection protocols }\end{array}$ & $\begin{array}{l}\text { - No facility for data pooling and } \\
\text { sharing }\end{array}$ & $\begin{array}{l}\text { - No facility to connect local } \\
\text { databases }\end{array}$ \\
\hline
\end{tabular}

Abbreviations: $\mathrm{AD}=$ Alzheimer's disease; DPUK = Dementias platform United Kingdom; EMIF-AD = European Medical Information Framework for Alzheimer's Disease; EPAD = European Prevention of Alzheimer's dementia consortium; JPND = Joint Programming Initiative Neurodegenerative Diseases 


\section{COLLABORATIONS AND FUTURE DIRECTIONS}

The EMIF-AD project facilitates the realization of large-scaled multicenter studies of existing $\mathrm{AD}$-related data. EMIF-AD infrastructures are currently being used by other projects such as the IMI project Real World Outcomes across the AD spectrum for better care (ROADMAP, https://roadmap-alzheimer.org), the project Alzheimer's biomarkers in daily practice (ABIDE), ${ }^{14} \mathrm{EPAD}$ and DPUK. In the future, we intent to connect with initiatives similar to EMIF-AD and provide an integrated portfolio of data sharing facilities worldwide. 


\section{REFERENCES}

1. Wilson RS, Segawa E, Boyle PA, Anagnos SE, Hizel LP, Bennett DA. The natural history of cognitive decline in Alzheimer's disease. Psychol Aging. 2012;27(4):1008-1017.

2. Barker WW, Luis CA, Kashuba A, et al. Relative frequencies of Alzheimer disease, Lewy body, vascular and frontotemporal dementia, and hippocampal sclerosis in the State of Florida Brain Bank. Alzheimer disease and associated disorders. 2002;16(4):203-212.

3. World Health Organization. World health statistics. Geneva, Switserland 2015.

4. Association As. 2017 Alzheimer's disease facts and figures. Alzheimer's \& Dementia. 2017;13(4):325-373.

5. Roberto G, Leal I, Sattar N, et al. Identifying Cases of Type 2 Diabetes in Heterogeneous Data Sources: Strategy from the EMIF Project. PloS one. 2016;11(8):e0160648.

6. Bastiao Silva LA, Dias C, van der Lei J, Oliveira JL. Architecture to Summarize Patient-Level Data Across Borders and Countries. Stud Health Technol Inform. 2015;216:687-690.

7. Vos SJ, Verhey F, Frolich L, et al. Prevalence and prognosis of Alzheimer's disease at the mild cognitive impairment stage. Brain : a journal of neurology. 2015;138(Pt 5):1327-1338.

8. Jansen WJ, Ossenkoppele R, Knol DL, et al. Prevalence of cerebral amyloid pathology in persons without dementia: a meta-analysis. Jama. 2015;313(19):1924-1938.

9. Bos I, Vos, S.J.B., Vandenberghe, R., Scheltens, P., Engelborghs, S., Frisoni, G., Molinuevo, J., Wallin, A., Lléo, A., Popp, J., Martinez-Lage, P., Baird, A., Dobson, R., Legido-Quigley, C., Bertram, L., Sleegers, K., Kate ten, M., Barkhof, F., Zetterberg, H., Lovestone, S., Streffer, J., Visser, P. . The EMIF-AD Multimodal Biomarker Discovery Study: Design, methods and cohort characteristics. Submitted.

10. Jansen WJ, Ossenkoppele R, Tijms BM, et al. Association of Cerebral Amyloid-beta Aggregation With Cognitive Functioning in Persons Without Dementia. JAMA Psychiatry. 2017.

11. Bos I, Vos SJ, Frölich L, et al. The frequency and influence of dementia risk factors in prodromal Alzheimer's disease. Neurobiology of aging. 2017.

12. Vermunt L, Veal, C.D, Meulen ter L., Chrysostomou, C., Flier van der, W., Frisoni, G.B., Guessous, I., Kivipelto, M., Marizzoni, M., Martinez-Lage, P., Molinuevo, JL., Porteus, D., Ritchie, K., Scheltens, P., Ousset, PJ., Ritchie, C.W., Luscan, G., Brookes, A.J., Visser, PJ. European Prevention of Alzheimer's Dementia (EPAD) Registry: recruitment and pre-screening approach for a longitudinal cohort and prevention trials. In press.

13. Toga AW, Neu SC, Bhatt P, Crawford KL, Ashish N. The Global Alzheimer's Association Interactive Network. Alzheimer's \& dementia : the journal of the Alzheimer's Association. 2015.

14. de Wilde A, van Maurik IS, Kunneman M, et al. Alzheimer's biomarkers in daily practice (ABIDE) project: Rationale and design. Alzheimers Dement (Amst). 2017;6:143-151. 


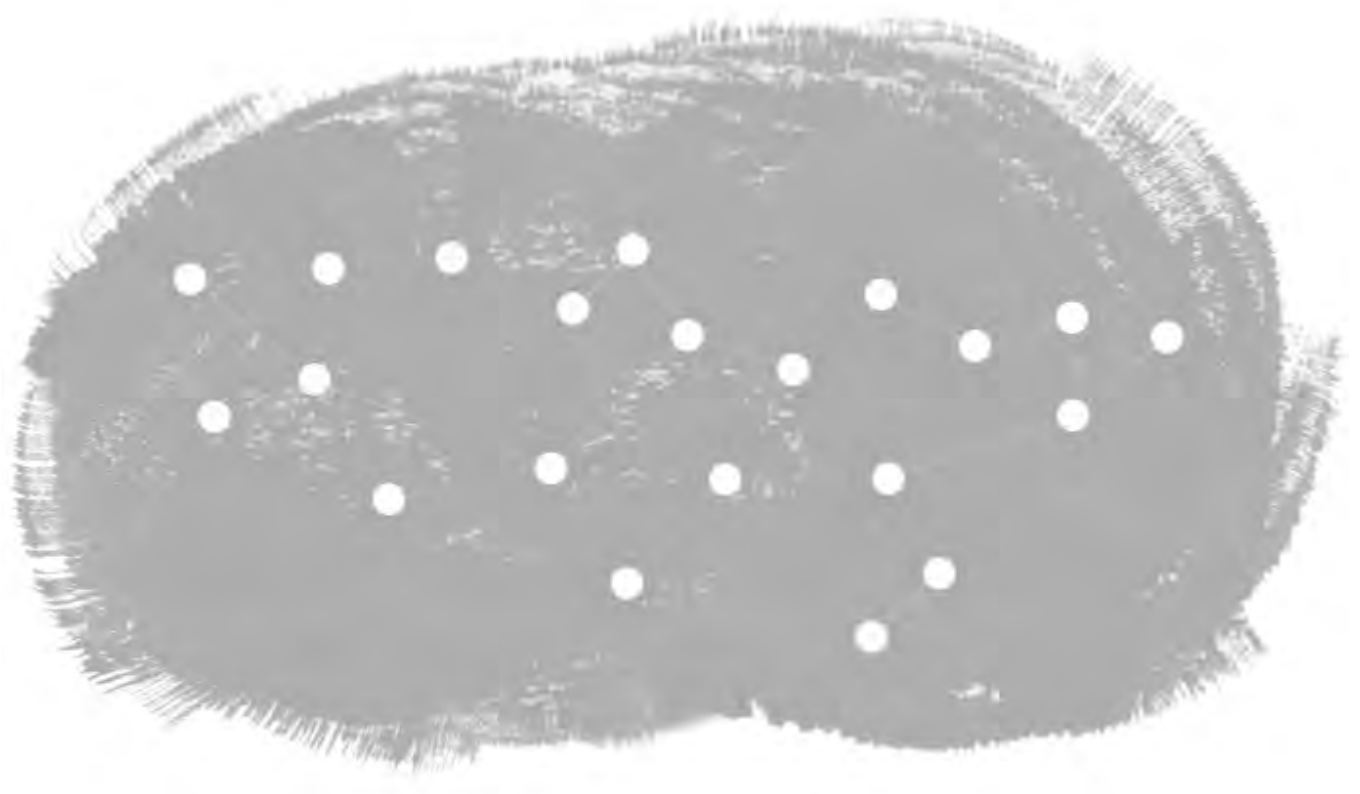




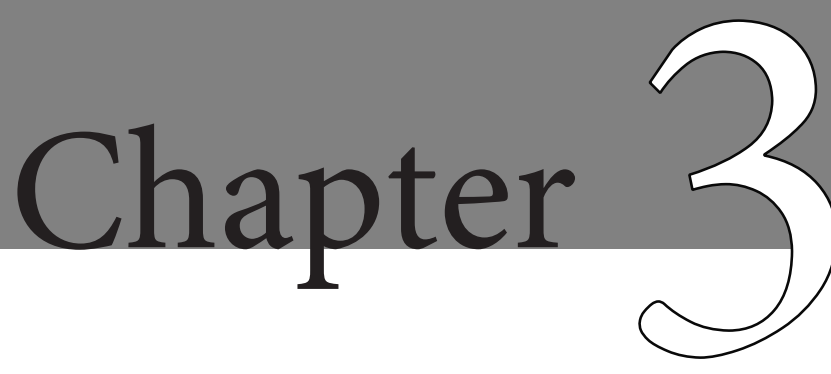

\section{The EMIF-AD Multimodal Biomarker Discovery Study: Design, methods and cohort characteristics}

Alzheimer's Research and Therapy 2018; 10:64

Isabelle Bos, Stephanie Vos, Rik Vandenberghe, Philip Scheltens, Sebastiaan Engelborghs, Giovanni Frisoni, José Luis Molinuevo, Anders Wallin, Alberto Lleó, Julius Popp, Pablo Martinez-Lage, Alison Baird, Richard Dobson, Cristina Legido-Quigley, Kristel Sleegers, Christine Van Broeckhoven, Lars Bertram, Mara ten Kate, Frederik Barkhof, Henrik Zetterberg, Simon Lovestone, Johannes Streffer and Pieter Jelle Visser 


\section{Chapter 3}

\section{ABSTRACT}

Background: There is an urgent need for novel, noninvasive biomarkers to diagnose Alzheimer's disease $(\mathrm{AD})$ in the pre-dementia stages and to predict rate of decline. Therefore, we set-up the European Medical Information Framework for Alzheimer's Disease Multimodal Biomarker Discovery (EMIF-AD MBD) study. In this report, we describe the design of the study, the methods used and the characteristics of the participants.

Methods: Participants were selected from existing multicenter and single center European studies. Inclusion criteria were having normal cognition (NC) or a diagnosis of mild cognitive impairment (MCI) or AD-type dementia at baseline, age above 50, known amyloid-beta $(A \beta)$ status, availability of cognitive test results and at least two of the following materials: plasma, DNA, magnetic resonance imaging (MRI) or cerebrospinal fluid (CSF). In plasma, targeted and untargeted metabolomic and proteomic analyses were performed and in CSF targeted and untargeted proteomics were performed. Genome-wide SNP genotyping, next generation sequencing and methylation profiling were conducted in DNA. Visual rating and volumetric measures were assessed on MRI. Baseline characteristics were analyzed using ANOVA or Chi-Square, rate of decline by linear mixed modeling.

Results: We included 1221 individuals (NC n=492, MCI n=527, AD-type dementia $n=202$ ) with a mean age of 67.9 (SD 8.3). The percentage $A \beta+$ was $26 \%$ in the NC, $58 \%$ in the MCI, and $87 \%$ in the AD-type dementia groups. Plasma samples were available for 1189 (97\%), DNA samples for 929 (76\%), MRI scans for 862 (71\%) and CSF samples for 767 (63\%) subjects. For 759 (62\%) individuals, clinical follow-up data were available. In each diagnostic group, the $A P O E \varepsilon 4$ allele was more frequent amongst $A \beta+$ individuals $(\mathrm{p}<0.001)$. Only in MCI, there was a difference in baseline MMSE score between the A $\beta$ groups $(\mathrm{p}<0.001)$. The $\mathrm{A} \beta+$ had a faster rate of decline on the MMSE during follow-up in the NC $(\mathrm{p}<0.001)$ and MCI $(\mathrm{p}<0.001)$ groups.

Conclusions: The characteristics of this large cohort of elderly subjects at various cognitive stages, confirm the central roles of $\mathrm{A} \beta$ and $A P O E \varepsilon 4$ in $\mathrm{AD}$ pathogenesis. The results of the multimodal analyses will provide new insights into underlying mechanisms and facilitate the discovery of new diagnostic and prognostic AD biomarkers. 


\section{INTRODUCTION}

Over the last decade great progress has been made in diagnosing Alzheimer's disease (AD) at an early disease stage, including before the onset of dementia. ${ }^{1,2}$ The biomarkers amyloid-beta (A $\beta$ ) and tau in cerebrospinal fluid (CSF) or amyloid and tau load via positron emission tomography (PET) have become indispensable in the $\mathrm{AD}$ research field, especially as part of clinical trials for disease modification and secondary prevention..$^{3-6}$ Nonetheless, a better understanding of the underlying pathophysiological disease mechanisms as well as the discovery of diagnostic and prognostic markers that are inexpensive and minimally invasive to obtain, would enhance the development of therapeutic interventions.

Currently, CSF and PET biomarkers are commonly used for the early diagnosis and prognosis of $\mathrm{AD} .^{7-9}$ However, PET imaging is fairly expensive, not universally available and both the procedures for obtaining CSF and PET are relatively invasive. Given this, complementing these highly specific biomarker modalities with markers in more readily accessible bio-fluids, would mark an important step forward. Consequently, many initiatives have been undertaken to discover and validate blood-based biomarkers for AD pathology, ${ }^{10,11}$ but so far results have been limited, due to small sample sizes, single modality analyses or other methodological issues. ${ }^{12}$ One critical issue so far has been the design (comparing individuals with $\mathrm{AD}$-type dementia with controls), which made the studies unsuitable for discovery of markers for the preclinical disease phase. To seek markers for the preclinical phase, a more sensitive and gradual approach has been proposed, described as the 'endophenotype approach' where discovery is predicted on a measure of pathology. ${ }^{13}$ Therefore, we designed the current study to enhance blood-based biomarker discovery by performing a series of omics techniques (e.g. proteomics, metabolomics, genomics), in a large cohort across the AD clinical disease spectrum, using an endophenotype approach.

This study was performed as a part of the European Medical Information Framework for Alzheimer's disease (EMIF-AD; http://www.emif.eu/). Funded through the Innovative Medicines Initiative (IMI), the EMIF project was established to facilitate the process of reusing and combining existing healthcare data with a focus on two therapeutic areas in the first instance: metabolic diseases and AD. One of the main aims of the EMIF-AD project is to accelerate the discovery of novel diagnostic and prognostic biomarkers for $\mathrm{AD}$ and to unravel the underlying pathophysiological mechanisms, using existing data and existing samples, that would otherwise be inaccessible to research beyond the project teams responsible for the collection. In this report, we will describe the set-up of the EMIF-AD Multimodal Biomarker Discovery (EMIF-AD MBD) study, the methods, as well as the characteristics of the included subjects. The results of the single and multimodal analyses will be described in future publications. 


\section{METHODS}

\section{General Outline}

In the EMIF-AD MBD study we used clinical data, samples, and scans that had already been collected as part of other cohort studies. We aimed to include a total of 1000 subjects across the clinical AD spectrum: 400 subjects with normal cognition (NC), 400 subjects with Mild Cognitive Impairment (MCI) and 200 subjects with mild AD-type dementia. To create a balanced design in terms of progression and to enable endophenotype designed biomarker studies, we intended to include $50 \% \mathrm{~A} \beta$ positive $(\mathrm{A} \beta+)$ and $50 \% \mathrm{~A} \beta$ negative $(\mathrm{A} \beta-)$ of the groups with NC and MCI. To conduct multimodal analyses, we initially aimed to include subjects who had material from MRI, plasma, DNA and CSF. Later, we adjusted this to subjects with material available in at least two of the modalities listed above.

\section{Selection of cohorts}

We used the EMIF-AD Catalogue (https://emif-catalogue.eu), established as part of the objective of EMIF which seeks to enable the finding, assessment and reutilization of preexisting data. The EMIF-AD Catalogue contains meta-data about European AD cohorts, enabling the selection of studies that included subjects who, in this instance, met the following inclusion criteria: 1) availability of data on A $\beta$ status, measured in CSF or by amyloid-positron emission tomography (PET); 2) age above 50 years at baseline; 3 ) and availability of MR scans, plasma and DNA samples. We identified 16 suitable cohorts. Two cohorts declined due to other research interests. Three cohorts were interested to collaborate, but unable because of legal and/or ethical restrictions, or unavailability of sufficient sample volumes. The 11 selected cohorts included three multicenter studies: EDAR $(n=204),{ }^{14}$ PharmaCog $(n=147)^{15}$ and DESCRIPA $(n=29)^{16}$ and eight single centers: Antwerp $(\mathrm{n}=149),{ }^{17}$ Amsterdam $(\mathrm{n}=172),{ }^{18}$ Barcelona Sant Pau $(\mathrm{n}=45),{ }^{19}$ Barcelona IDIBAPS $(n=120),{ }^{20}$ Leuven $(n=180),{ }^{21}$ San Sebastian GAP $(n=40),{ }^{22}$ Gothenburg $(n=95){ }^{23}$ and Lausanne $(n=40) .{ }^{24}$ Of these 11 cohorts, DESCRIPA, EDAR, PharmaCog, Amsterdam, Antwerp and Gothenburg were linked to partners in EMIF-AD, while the other 5 cohorts participated as affiliated data providers (ADP). All cohorts (e.g. partners and ADP) signed a material transfer agreement. The ADP also agreed to the EMIF project agreement. The local medical ethical committee in each center approved the study. Subjects had already provided written informed consent at time of inclusion in the cohort for use of data, samples and scans. Figure 1 shows a timeline of the different the events in establishing this cohort, from the search in the EMIF catalogue to the wet-lab analyses. 


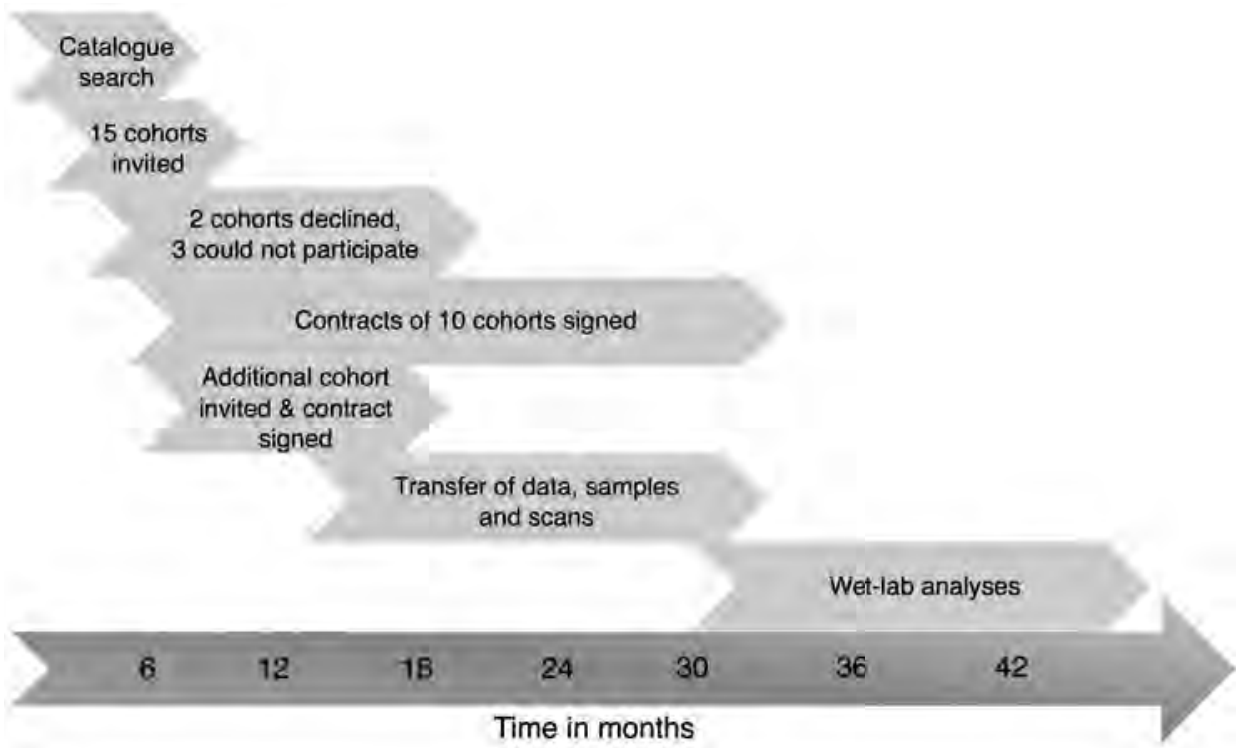

Figure 1. Timeline of events related to the EMIF-AD MBD study.

This diagram shows an estimation of timeline of different events related to the EMIF-AD MBD study.

\section{Baseline diagnoses}

In all cohorts, the definition for NC was a normal performance on neuropsychological assessment (within 1.5 SD of the average for age, gender and education). Five cohorts also used a score of 0 on the clinical dementia rating (CDR), ${ }^{25}$ and a single cohort used a cutoff $<3$ on the Global Deterioration Scale, ${ }^{26}$ to determine NC. Diagnosis of MCI was made according to the criteria of Petersen et al. ${ }^{27}$ in nine cohorts. Two cohorts used the Winblad criteria $^{28}$ to diagnose MCI. All cohorts used the National Institute of Neurological and Communicative Disorders and Stroke - Alzheimer's Disease and Related Disorders Association criteria (NINCDS-ADRDA) criteria $^{29}$ to diagnose AD-type dementia. Supplemental Table 1 lists the diagnostic criteria used per center.

\section{Clinical data}

All cohorts were asked to contribute available data on demographics, clinical information, neuropsychological testing and A $\beta$ status, as listed in Table 1. Medication use and comorbidities were classified into a number of categories, for which we created dichotomous variables (Table 1). Cognitive data was collected in all cohorts. The cognitive tests used varied across centers. Only the Mini Mental State Examination (MMSE) was administered in all centers and was available in nearly all subjects $(n=1216)$. 
Chapter 3

Table 1. Clinical dataset

\begin{tabular}{ll}
\hline Demographics & \\
\hline Age \\
Gender \\
Years of education \\
\hline Clinical information & \\
\hline
\end{tabular}

Diagnosis

Medication use

- Cardiovascular medication

- Dementia medication

- Hormonal medication

- Psychopharmaceuticals

- Other medication

Co-morbidities

- Cardiovascular disorders

- Cardiovascular risk factors

- Cerebrovascular disorders

- Endocrine disorders

- Neurological disorders

- Other cardiac disorders

- Psychiatric disorders

- Somatic disorders

Family history of dementia

- $\quad$ First degree relatives

- $\quad$ Second degree relatives

Functional impairment rating

\begin{tabular}{ll}
\hline Neuropsychological tests & \\
\hline & Memory, preferred test: AVLT \\
& Language, preferred test: Animal fluency \\
& Attention, preferred test: Trail Making Test A \\
& Executive Functioning, preferred test: Trail Making Test B \\
& Visuoconstruction, preferred test: Rey complex figure copy \\
\hline A $\beta$ measure & \\
\hline & CSF A $\beta_{42}$ value and local cut-off point \\
& Amyloid PET SUV and local cut-off point
\end{tabular}

${ }^{*}$ At least one A $\beta$ measure. Abbreviations: AVLT = Auditory Verbal Learning Test, CSF = Cerebrospinal Fluid, $\mathrm{A} \beta=$ amyloid-beta, $\mathrm{PET}=$ Positron emission tomography, SUV $=$ standardized uptake value .

We requested at least one test from the following cognitive domains: memory, language, attention, executive functioning and visuo-construction. ${ }^{16}$ For each cognitive domain, we selected a primary test (Table 1). If the preferred tests were not available, we selected an 
alternative priority test from the same cognitive domain. Supplemental Table 2 provides an overview of the different tests used for each cognitive domain. For each test, we requested the raw scores and if available, $\mathrm{z}$-scores calculated based on local normative data. If local normative data was unavailable, we calculated z-scores based on published normative data from healthy controls for that test. Per cognitive domain we combined $z$ scores which we used as a continuous variable, and we used a cut-off of $z$-score $<-1.5$ to define abnormality. Clinical data was harmonized, pooled and stored onto an online data platform using tranSMART, ${ }^{30}$ now enriched for dementia research purposes through the EMIF-AD project.

\section{Plasma analyses}

Initially, the minimum requested amount of plasma was 0.7 milliliters ( $\mathrm{ml}$ ). If available, another $0.7 \mathrm{ml}$ was requested to conduct additional analyses in a subgroup of subjects. In some cases only $0.5 \mathrm{ml}$ was available. Prior to the analyses samples were checked visually on consistency and volume. Results of analyses were also quality checked by inspecting patterns of outliers, and excluding consistent outliers across analyses. Analyses conducted on these samples include: 1) targeted analyses of plasma proteins previously identified ${ }^{31}$ and confirmed in at least one replication study, a panel of complement proteins nominated because of increasing evidence from genomics of the role of innate immunity in $\mathrm{AD}$ and analysis of plasma neurofilament light (NFL) chain; 2) Untargeted proteomic analyses using aptamer capture approaches; 3) Untargeted and Targeted metabolic analyses using a 883-metabolite panel for the targeted assay.

\section{Genetic analyses}

A total amount of 2.6 microgram DNA or $1 \mathrm{ml}$ whole blood, from which DNA was to be extracted, was requested for the genetic analyses. After performing routine quality checks on extracted DNA (e.g. agarose gel electrophoresis, determination of A260/280 and A260/230 ratios, PicoGreen quantification), we performed three types of assessments on each sample passing quality control: genome-wide SNP genotyping (Global Screening Array, Illumina, Inc.), genome-wide DNA methylation profiling (Infinium MethylationEPIC BeadChip, Illumina, Inc.) and whole exome sequencing.

\section{CSF analyses}

The requested amount of CSF to conduct all planned analyses was $0.4 \mathrm{ml}$ and this was used for untargeted proteomic and peptidomic analyses, and a number of targeted analyses measuring concentrations of: $A \beta_{38}, A \beta_{40}, A \beta_{42}, A \beta_{42 / 40}$, YKL-40, NFL and neurogranin $(\mathrm{Ng})$. Prior to the analyses, CSF samples were checked visually on volume and absence of blood contamination. 


\section{MRI analyses}

MRI scans were assembled centrally, quality checked and visually assessed by a single rater. T1-weigthed and when available FLAIR and/or T2*/SWI images were used for qualitative visual rating, including medial temporal lobe atrophy, ${ }^{32}$ global cortical atrophy, ${ }^{33}$ white matter hyperintensities ${ }^{34}$ and microbleeds (defined as small $(<10 \mathrm{~mm})$ round foci of hypointense signal in brain parenchyma). 3D T1 scans were uploaded to the Neurgrid platform (https://neugrid4you.eu) ${ }^{35}$, for storage and automated quantitative analyses. Volumetric analysis included assessment of hippocampal and whole brain volume and cortical thickness.

\section{Amyloid classification}

$\mathrm{A} \beta$ status was defined by the CSF $\mathrm{A} \beta_{42 / 40}$ of the central analyses, using a cut-off of $<0.061$ to determine abnormality ( $n=770)$. The cut-off for the $A \beta_{42 / 40}$ ratio was determined based on mixture model analyses comparing the $\mathrm{CN}$ and $\mathrm{AD}$ groups in this dataset. When no CSF was contributed for central analyses, the local CSF $A \beta_{42}$ value $(n=271)$ or the standardized uptake value ratio (SUVR) on an amyloid-PET scan $(n=180)$ with local cut-off values to determine abnormality, were used (Supplemental Table 3).

\section{Statistical analyses}

Baseline characteristics were compared between groups using ANOVA for continuous variables and Chi-square for categorical variables. General linear mixed models, with random intercepts and slopes for each subject were used to examine the influence of $A \beta$ status on MMSE performance and decline over time, adjusted for age, gender and years of education. Missing values for APOE genotype $(n=12)$ and years of education $(n=105)$ were imputed using regression within study with at least two significant predictors (i.e. age, gender, MMSE etc.). Statistical analyses were performed using R Statistical Software (version 3.3.3) and SPSS (version 24), with significance defined as $\mathrm{p}<0.05$.

\section{RESULTS}

We initially sought to identify 1000 individuals with data available in all modalities. However, because not all studies could contribute data for all modalities, we included more participants to meet the aimed number of individuals for each modality. In total, 1221 subjects were included in the study, with a mean age of 67.9 (SD 8.3) years. Six hundred and sixty-seven (54\%) were female and the average education level was 11.7 (SD 4.1) years. At baseline, 492 (40\%) had NC, 527 (43\%) had a MCI diagnosis and 202 (17\%) had a clinical diagnosis of AD-type dementia. For 758 (62\%) individuals there was follow-up 
data available (e.g. at least a clinical diagnosis or MMSE at follow-up): 217 (44\%) NC, 398 (76\%) MCI and 143 (71\%) demented subjects. The average follow-up time for all 758 individuals was 2.3 (SD 1.2) years. Per diagnostic groups the average clinical follow-up time was: CN 2.4 (SD 0.9), MCI 2.2 (SD 1.3) and AD 2.2 (SD 1.4) years.

Table 2: Baseline characteristics by clinical diagnosis and $\mathrm{A} \beta$ status

\begin{tabular}{|c|c|c|c|c|c|c|c|}
\hline \multirow[b]{2}{*}{ Characteristics } & \multirow[b]{2}{*}{$\mathrm{n}$ total } & \multicolumn{2}{|c|}{ Normal cognition } & \multicolumn{2}{|c|}{ MCI } & \multicolumn{2}{|c|}{$\mathrm{AD}$ dementia } \\
\hline & & $\begin{array}{l}A \beta- \\
n=365\end{array}$ & $\begin{array}{l}\mathrm{A} \beta+ \\
\mathrm{n}=127\end{array}$ & $\begin{array}{l}A \beta- \\
n=220\end{array}$ & $\begin{array}{l}A \beta+ \\
n=307\end{array}$ & $\begin{array}{l}A \beta- \\
n=27\end{array}$ & $\begin{array}{l}\mathrm{A} \beta+ \\
\mathrm{n}=175\end{array}$ \\
\hline Age, years & 1221 & $64.4(7.6)$ & $66.9(7.9)^{\star \star}$ & $68.3(8.2)$ & $70.7(7.4)^{\star * *}$ & $73.0(8.4)$ & $69.9(8.8)$ \\
\hline Female, $\mathrm{n}$ & 1221 & $203(56)$ & $66(52)$ & $108(49)$ & $172(56)$ & $12(44)$ & $96(55)$ \\
\hline Education, years & 1221 & $13.5(3.7)$ & $12.7(4.0)^{\star}$ & $10.6(3.8)$ & $10.8(3.7)$ & $8.5(4.4)$ & $10.6(3.8)^{* *}$ \\
\hline APOE- $\varepsilon 4$ carrier, $n$ & 1221 & $122(33)$ & $76(60)^{\star * *}$ & $46(21)$ & $200(65)^{\star * * *}$ & $7(26)$ & $114(65)^{* * *}$ \\
\hline $\begin{array}{l}\text { Mean follow-up time, } \\
\text { years }\end{array}$ & 758 & $2.3(0.8)$ & $2.5(1.1)$ & $2.2(1.3)$ & $2.2(1.3)$ & $1.7(0.9)$ & $2.2(1.4)$ \\
\hline MMSE score & 1215 & $28.9(1.1)$ & $28.8(1.2)$ & $27.0(2.3)$ & $25.9(2.7)^{\star * *}$ & $21.5(5.4)$ & $21.7(4.6)$ \\
\hline $\begin{array}{l}\text { Memory delayed z- } \\
\text { score }\end{array}$ & 1049 & $0.1(1.1)$ & $0.0(1.2)$ & $-0.9(1.3)$ & $-1.4(1.4)^{\star * *}$ & $-2.2(1.2)$ & $-2.4(1.1)$ \\
\hline Language $\mathrm{z}$-score & 1181 & $-0.2(1.0)$ & $-0.1(1.0)$ & $-0.7(1.2)$ & $-1.0(2.0)$ & $-1.9(1.2)$ & $-2.3(2.4)$ \\
\hline Attention $\mathrm{z}$-score & 1128 & $0.3(1.1)$ & $0.2(0.9)$ & $-1.0(1.8)$ & $-1.0(1.8)$ & $-2.1(2.5)$ & $-2.1(2.0)$ \\
\hline $\begin{array}{l}\text { Executive functioning } \\
\text { z-score }\end{array}$ & 976 & $0.3(1.1)$ & $0.1(1.1)$ & $-0.9(1.9)$ & $-1.4(2.1)^{*}$ & $-1.2(2.5)$ & $-3.4(2.8)^{\star}$ \\
\hline $\begin{array}{l}\text { Visuoconstruction } \mathrm{z}- \\
\text { score }\end{array}$ & 664 & $0.2(1.4)$ & $0.2(0.8)$ & $-0.3(1.7)$ & $-0.4(1.8)$ & $-2.1(2.4)$ & $-1.3(2.0)$ \\
\hline
\end{tabular}

Results are mean (SD) or continuous variables or frequency (\%). Abbreviations: APOE = Apolipoprotein E, $\mathrm{MCI}=$ Mild Cognitive Impairment, MMSE $=$ Mini Mental State Examination. ${ }^{*} \mathrm{p}<0.05,{ }^{* *} \mathrm{p}<0.01,{ }^{* * *} \mathrm{p}<0.001$ in comparison to $\mathrm{A} \beta$ - group.

Table 2 shows the baseline characteristics of the sample by $\mathrm{A} \beta$ status and by baseline diagnosis. In the $\mathrm{NC}$ and $\mathrm{MCI}$ groups, the $\mathrm{A} \beta+$ subjects were older than the $\mathrm{A} \beta$ - subjects (NC: $\mathrm{p}=0.002 ; \mathrm{MCI}, \mathrm{p}<0.001)$. In all diagnostic groups, $\mathrm{A} \beta+$ subjects were more likely to be APOE- $\varepsilon 4$ carrier (all p-values $<0.001$ ). In the MCI subjects only, there was a difference on baseline MMSE score between the A $\beta$ groups ( $\mathrm{p}=0.001$ ). Regarding cognitive domains, we found differences in memory $(\mathrm{p}<0.001)$ and executive functioning $(\mathrm{p}=0.042) \mathrm{z}$-scores in individuals with MCI. In individuals with AD-type dementia we found that $A \beta+$ individuals performed worse on an executive functioning task $(\mathrm{p}=0.013)$.

Table 3 shows the number of subjects per modality by diagnostic category. Plasma samples were contributed for 1189 (97\%), DNA for 929 (76\%), MR scans for 862 (71\%) and CSF for 770 (63\%) subjects. There were $482(40 \%)$ subjects that contributed material in all modalities. Of this subsample, 89 (18\%) had NC, 318 (66\%) MCI and 75 (16\%) had a diagnosis of $\mathrm{AD}$-type dementia at baseline. 
Chapter 3

Table 3: Number of subjects from different cohorts for each modality by diagnosis

\begin{tabular}{|c|c|c|c|c|c|c|}
\hline Cohort & Diagnosis & Clinical data & Plasma & DNA & MRI & CSF \\
\hline \multirow[t]{3}{*}{ Amsterdam } & $\mathrm{NC}$ & 30 & 29 & 26 & 30 & 30 \\
\hline & MCI & 82 & 80 & 68 & 82 & 82 \\
\hline & $\mathrm{AD}$ dementia & 60 & 60 & 53 & 60 & 60 \\
\hline \multirow[t]{2}{*}{ Antwerp } & MCI & 103 & 100 & 101 & 50 & 103 \\
\hline & AD dementia & 46 & 47 & 46 & 0 & 46 \\
\hline \multirow[t]{2}{*}{ DESCRIPA } & $\mathrm{NC}$ & 12 & 12 & 8 & 5 & 12 \\
\hline & MCI & 17 & 17 & 12 & 9 & 17 \\
\hline \multirow[t]{3}{*}{ EDAR } & $\mathrm{NC}$ & 48 & 47 & 42 & 14 & 47 \\
\hline & MCI & 77 & 75 & 65 & 24 & 75 \\
\hline & AD dementia & 79 & 78 & 69 & 19 & 76 \\
\hline GAP & $\mathrm{NC}$ & 40 & 40 & 40 & 38 & 40 \\
\hline \multirow[t]{2}{*}{ Gothenburg } & $\mathrm{NC}$ & 49 & 48 & - & 48 & - \\
\hline & MCI & 46 & 44 & - & 46 & - \\
\hline \multirow[t]{3}{*}{ IDIBAPS } & NC & 76 & 77 & - & 40 & - \\
\hline & MCI & 27 & 27 & - & 14 & - \\
\hline & AD dementia & 17 & 16 & - & 14 & - \\
\hline \multirow[t]{2}{*}{ Lausanne } & $\mathrm{NC}$ & 12 & 12 & 12 & 12 & 12 \\
\hline & MCI & 28 & 28 & 28 & 27 & 28 \\
\hline Leuven & $\mathrm{NC}$ & 180 & 163 & 168 & 179 & - \\
\hline Pharmacog & MCI & 147 & 144 & 146 & 147 & 147 \\
\hline Sant Pau & $\mathrm{NC}$ & 45 & 45 & 45 & - & - \\
\hline \multirow[t]{4}{*}{ Total } & $\mathrm{NC}$ & 492 & 473 & 341 & 366 & 141 \\
\hline & MCI & 527 & 515 & 420 & 399 & 452 \\
\hline & AD dementia & 202 & 201 & 168 & 100 & 182 \\
\hline & Overall & 1221 & 1189 & 926 & 865 & 775 \\
\hline
\end{tabular}

Abbreviations: $\mathrm{CSF}=$ cerebrospinal fluid, $\mathrm{DNA}=$ Deoxyribonucleic acid, $\mathrm{MCI}=$ Mild Cognitive Impairment, MRI = magnetic resonance imaging, $\mathrm{NC}=$ Normal cognition.

Table 4: Effect of A $\beta$ on MMSE scores over time by diagnostic group

\begin{tabular}{llllll}
\hline Diagnosis & $\mathrm{n}$ & Baseline & $\mathrm{p}$-value & Slope & $\mathrm{p}$-value \\
\hline NC & 482 & $-0.35 \pm 0.20$ & 0.170 & $-0.60 \pm 0.13$ & $<0.001$ \\
MCI & 459 & $-1.56 \pm 0.24$ & $<0.001$ & $-0.60 \pm 0.14$ & $<0.001$ \\
AD dementia & 162 & $-0.05 \pm 1.05$ & 0.965 & $-0.21 \pm 0.64$ & 0.742 \\
\hline
\end{tabular}

Numbers are linear mixed model coefficients \pm standard error, relative to the $A \beta$-group, adjusted for age, gender and years of education. Abbreviations: $\mathrm{MCI}=$ Mild Cognitive Impairment, $\mathrm{NC}=$ Normal cognition. 
Table 4 and Figure 2 show the effect of A $\beta$ on MMSE scores over time for each diagnostic group, adjusted for demographics. At baseline, there is only a difference in MMSE for the MCI group $(\mathrm{p}<0.001)$. In the NC and MCI groups, the $\mathrm{A} \beta+$ individuals in the NC and MCI group decline at a faster rate than the $\mathrm{A} \beta$ - individuals (NC: $\mathrm{p}<0.001 ; \mathrm{MCI} p<0.001$ ). For the demented subjects, $A \beta$ did not influence the rate of decline (Table 4, Figure 2).

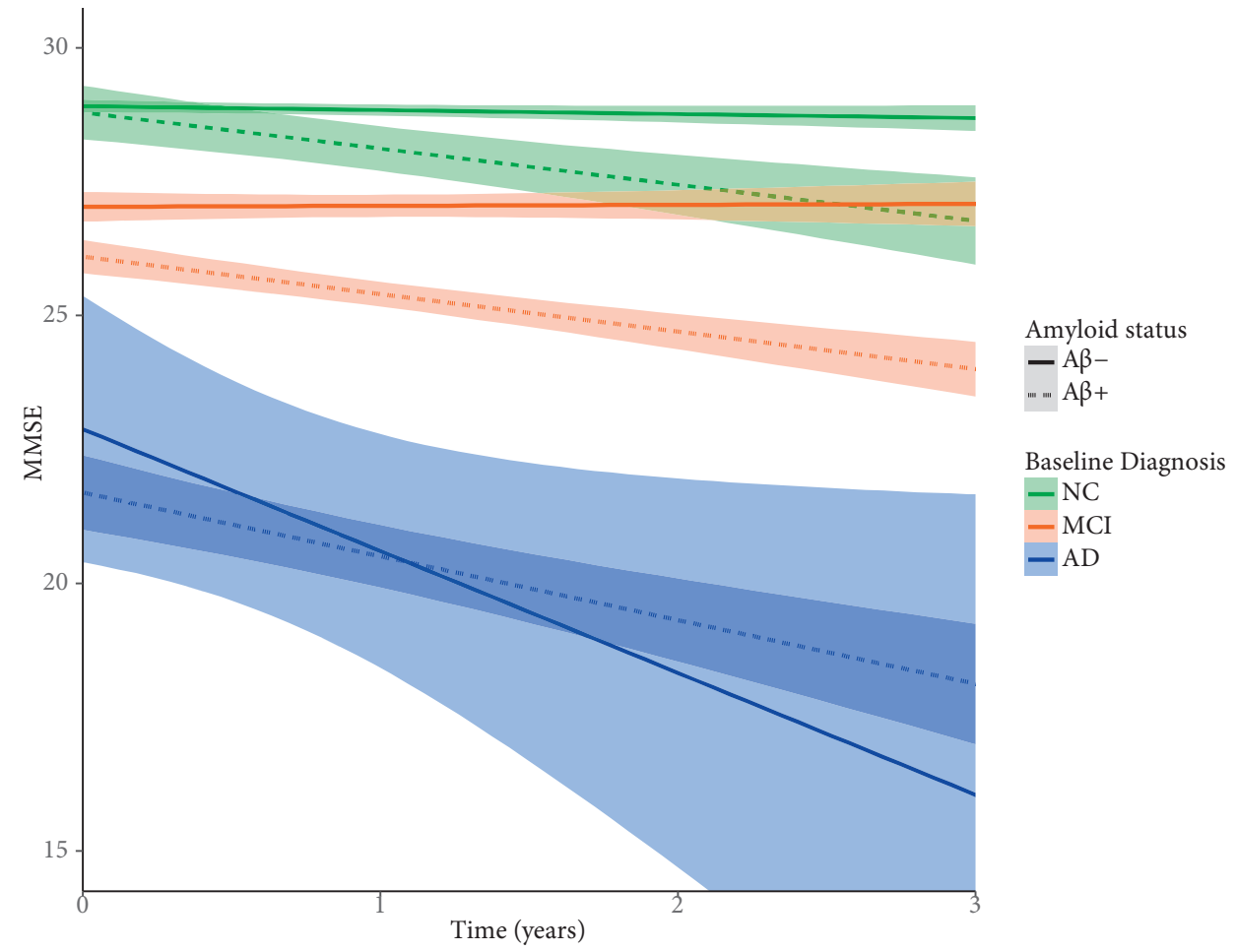

Figure 2. MMSE scores over time by A $\beta$ status and diagnosis.

The graph shows the mean scores and 95\% confidence intervals of the MMSE over time for the three diagnostic groups and by $A \beta$ status, adjusted for demographics. The solid lines represent the $A \beta$-groups and the dashed lines represent the $A \beta+$ groups.

\section{DISCUSSION}

The aim of the EMIF consortium is to enable the reutilization of pre-existing data including the finding and assessment of relevant datasets and facilitation of their interoperability and reuse. For the EMIF-AD component, a major use-case objective has been to use the processes and tools established in the consortium to generate a novel cross-cohort data and sample collection for the discovery and validation of biomarkers for use in clinical trials using a multimodal and endophenotype design. The first results presented in 
this report confirm the central roles of $\mathrm{A} \beta$ and $A P O E \varepsilon 4$ in the pathogenesis of $\mathrm{AD}$ which is consistent with findings from other large cohorts. ${ }^{36,37}$ The molecular studies are ongoing and will be reported in future publications.

$\mathrm{AD}$ is a complex and multifactorial disorder, which underscores the need for multimodal studies with sufficient statistical power. ${ }^{38}$ To date these large studies are scarce, especially those including subjects across the whole clinical AD spectrum. To our knowledge, the only other large-scale studies that collected plasma, DNA, CSF and imaging material from individuals in various cognitive stages, are the Alzheimer's Disease Neuroimaging Initiative $(\mathrm{ADNI})^{36}$ and the Australian Imaging, Biomarkers and Lifestyle study of ageing $(\mathrm{AIBL})^{37}$ studies. Since these datasets are so unique, findings from these studies are difficult to validate. The current study will not only be of great additional value due to its explorative nature and complementary lab analyses, but also because previous findings can be validated in a large size cohort with multimodal data. We collected a wide variety of clinical variables including neuropsychological tests, comorbidities, medication use and psychiatric questionnaires. All this clinical data is harmonized, pooled and stored on an online, secure data platform (tranSMART) and is available upon request to answer a wide range of $\mathrm{AD}$ related research questions.

Besides the major advantages, this study also has some limitations. Currently we do not have clinical follow-up data for all subjects, as some centers are still in the process of collecting this. However, this data may be added to the database in the future. Also, the data, samples and scans contributed to this study were collected at different centers and were not collected using the same protocol, which will lead to pre-analytical variability. To limit this variability, the samples were analyzed centrally and the clinical data was harmonized using standardized values and dichotomous variables.

In conclusion, the various complementary analyses conducted in plasma, DNA, CSF and on MR images in a large-sized cohort of individuals across the clinical AD spectrum provide a unique opportunity to discover novel diagnostic and prognostic markers and will also increase knowledge into the $\mathrm{AD}$ pathophysiology, which is required for the development of novel therapeutic interventions. 


\section{REFERENCES}

1. Jack CR, Knopman DS, Jagust WJ, et al. Tracking pathophysiological processes in Alzheimer's disease: an updated hypothetical model of dynamic biomarkers. The Lancet Neurology. 2013;12(2):207-216.

2. Bateman RJ, Xiong C, Benzinger TL, et al. Clinical and biomarker changes in dominantly inherited Alzheimer's disease. The New England journal of medicine. 2012;367(9):795-804.

3. Sperling R, Mormino E, Johnson K. The evolution of preclinical Alzheimer's disease: implications for prevention trials. Neuron. 2014;84(3):608-622.

4. Mattsson N, Carrillo MC, Dean RA, et al. Revolutionizing Alzheimer's disease and clinical trials through biomarkers. Alzheimers Dement (Amst). 2015;1(4):412-419.

5. Hampel H, Frank R, Broich K, et al. Biomarkers for Alzheimer's disease: academic, industry and regulatory perspectives. Nature reviews Drug discovery. 2010;9(7):560-574.

6. Vandenberghe R, Adamczuk K, Dupont P, Laere KV, Chetelat G. Amyloid PET in clinical practice: Its place in the multidimensional space of Alzheimer's disease. Neuroimage Clin. 2013;2:497-511.

7. Scheltens P, Blennow K, Breteler MM, et al. Alzheimer's disease. Lancet. 2016;388(10043):505-517.

8. Duits FH, Prins ND, Lemstra AW, et al. Diagnostic impact of CSF biomarkers for Alzheimer's disease in a tertiary memory clinic. Alzheimer's \& dementia : the journal of the Alzheimer's Association. 2015;11(5):523-532.

9. Rathore S, Habes M, Iftikhar MA, Shacklett A, Davatzikos C. A review on neuroimaging-based classification studies and associated feature extraction methods for Alzheimer's disease and its prodromal stages. NeuroImage. 2017;155:530-548.

10. Laske C. Blood-based biomarkers in Alzheimer disease: where are we now and where have we to go? JAMA Neurol. 2013;70(1):133.

11. Snyder HM, Carrillo MC, Grodstein F, et al. Developing novel blood-based biomarkers for Alzheimer's disease. Alzheimer's \& dementia : the journal of the Alzheimer's Association. 2014;10(1):109-114.

12. Galasko D, Golde TE. Biomarkers for Alzheimer's disease in plasma, serum and blood - conceptual and practical problems. Alzheimer's research \& therapy. 2013;5(2):10.

13. Baird AL, Westwood S, Lovestone S. Blood-Based Proteomic Biomarkers of Alzheimer's Disease Pathology. Front Neurol. 2015;6:236.

14. Reijs BLR, Ramakers I, Elias-Sonnenschein L, et al. Relation of Odor Identification with Alzheimer's Disease Markers in Cerebrospinal Fluid and Cognition. Journal of Alzheimer's disease : JAD. 2017;60(3):1025-1034.

15. Galluzzi S, Marizzoni M, Babiloni C, et al. Clinical and biomarker profiling of prodromal Alzheimer's disease in workpackage 5 of the Innovative Medicines Initiative PharmaCog project: a 'European ADNI study'. Journal of internal medicine. 2016;279(6):576-591.

16. Visser PJ, Verhey FR, Boada M, et al. Development of screening guidelines and clinical criteria for predementia Alzheimer's disease. The DESCRIPA Study. Neuroepidemiology. 2008;30(4):254-265.

17. Somers C, Struyfs H, Goossens J, et al. A Decade of Cerebrospinal Fluid Biomarkers for Alzheimer's Disease in Belgium. Journal of Alzheimer's disease : JAD. 2016;54(1):383-395.

18. van der Flier WM, Pijnenburg YA, Prins N, et al. Optimizing patient care and research: the Amsterdam Dementia Cohort. Journal of Alzheimer's disease : JAD. 2014;41(1):313-327.

19. Alcolea D, Carmona-Iragui M, Suarez-Calvet M, et al. Relationship between beta-Secretase, inflammation and core cerebrospinal fluid biomarkers for Alzheimer's disease. Journal of Alzheimer's disease : JAD. 2014;42(1):157-167.

20. Fortea J, Sala-Llonch R, Bartres-Faz D, et al. Increased cortical thickness and caudate volume precede atrophy in PSEN1 mutation carriers. Journal of Alzheimer's disease : JAD. 2010;22(3):909-922. 
21. Adamczuk K, Schaeverbeke J, Nelissen N, et al. Amyloid imaging in cognitively normal older adults: comparison between (18)F-flutemetamol and (11)C-Pittsburgh compound B. European journal of nuclear medicine and molecular imaging. 2016;43(1):142-151.

22. Estanga A, Ecay-Torres M, Ibanez A, et al. Beneficial effect of bilingualism on Alzheimer's disease CSF biomarkers and cognition. Neurobiology of aging. 2017;50:144-151.

23. Wallin A, Nordlund A, Jonsson M, et al. Alzheimer's disease--subcortical vascular disease spectrum in a hospital-based setting: Overview of results from the Gothenburg MCI and dementia studies. J Cereb Blood Flow Metab. 2016;36(1):95-113.

24. Tautvydaite D, Kukreja D, Antonietti JP, Henry H, von Gunten A, Popp J. Interaction between personality traits and cerebrospinal fluid biomarkers of Alzheimer's disease pathology modulates cognitive performance. Alzheimer's research \& therapy. 2017;9(1):6.

25. Morris JC. The Clinical Dementia Rating (CDR): current version and scoring rules. Neurology. 1993;43(11):2412-2414

26. Reisberg B, Ferris SH, de Leon MJ, Crook T. The Global Deterioration Scale for assessment of primary degenerative dementia. The American journal of psychiatry. 1982.

27. Petersen RC. Mild cognitive impairment as a diagnostic entity. Journal of internal medicine. 2004;256(3):183-194.

28. Winblad B, Palmer K, Kivipelto M, et al. Mild cognitive impairment--beyond controversies, towards a consensus: report of the International Working Group on Mild Cognitive Impairment. Journal of internal medicine. 2004;256(3):240-246.

29. McKhann G, Drachman D, Folstein M, Katzman R, Price D, Stadlan EM. Clinical diagnosis of Alzheimer's disease: report of the NINCDS-ADRDA Work Group under the auspices of Department of Health and Human Services Task Force on Alzheimer's Disease. Neurology. 1984;34(7):939-944.

30. Scheufele E, Aronzon D, Coopersmith R, et al. tranSMART: An Open Source Knowledge Management and High Content Data Analytics Platform. AMIA Jt Summits Transl Sci Proc. 2014;2014:96-101.

31. Hye A, Riddoch-Contreras J, Baird AL, et al. Plasma proteins predict conversion to dementia from prodromal disease. Alzheimer's \& dementia : the journal of the Alzheimer's Association. 2014;10(6):799807.e792.

32. Scheltens P, Leys D, Barkhof F, et al. Atrophy of medial temporal lobes on MRI in "probable" Alzheimer's disease and normal ageing: diagnostic value and neuropsychological correlates. Journal of neurology, neurosurgery, and psychiatry. 1992;55(10):967-972.

33. Pasquier F, Leys D, Weerts JG, Mounier-Vehier F, Barkhof F, Scheltens P. Inter- and intraobserver reproducibility of cerebral atrophy assessment on MRI scans with hemispheric infarcts. Eur Neurol. 1996;36(5):268-272.

34. Koedam EL, Lehmann M, van der Flier WM, et al. Visual assessment of posterior atrophy development of a MRI rating scale. European radiology. 2011;21(12):2618-2625.

35. Redolfi A, Bosco P, Manset D, Frisoni GB. Brain investigation and brain conceptualization. Funct Neurol. 2013;28(3):175-190.

36. Mueller SG, Weiner MW, Thal LJ, et al. The Alzheimer's disease neuroimaging initiative. Neuroimaging clinics of North America. 2005;15(4):869-877, xi-xii.

37. Ellis KA, Bush AI, Darby D, et al. The Australian Imaging, Biomarkers and Lifestyle (AIBL) study of aging: methodology and baseline characteristics of 1112 individuals recruited for a longitudinal study of Alzheimer's disease. International psychogeriatrics / IPA. 2009;21(4):672-687.

38. Perrin RJ, Fagan AM, Holtzman DM. Multimodal techniques for diagnosis and prognosis of Alzheimer's disease. Nature. 2009;461(7266):916-922. 


\section{SUPPLEMENTAL DATA}

Supplemental Table 1. Diagnostic criteria per cohort

\begin{tabular}{|c|c|c|c|c|c|}
\hline \multirow[b]{2}{*}{ Cohort } & \multirow[b]{2}{*}{ Country } & \multirow[b]{2}{*}{$\mathrm{n}$} & \multicolumn{3}{|c|}{ Diagnostic criteria } \\
\hline & & & $\mathrm{CN}$ & MCI & $\begin{array}{l}\text { AD-type } \\
\text { dementia }\end{array}$ \\
\hline Amsterdam & $\begin{array}{l}\text { The } \\
\text { Netherlands }\end{array}$ & 172 & $\begin{array}{l}>-1.5 \mathrm{SD} \text { on } \mathrm{NPE} \text { and } \mathrm{CDR}=0 \\
\text { or } 0.5\end{array}$ & Petersen criteria $^{1}$ & $\begin{array}{l}\text { NINCDS- } \\
\text { ADRA }^{3}\end{array}$ \\
\hline Antwerp & Belgium & 149 & $>-1.5 \mathrm{SD}$ on $\mathrm{NPE}$ & Petersen criteria ${ }^{1}$ & $\begin{array}{l}\text { NINCDS- } \\
\text { ADRA }^{3}\end{array}$ \\
\hline DESCRIPA & $\begin{array}{l}\text { European } \\
\text { multicenter }\end{array}$ & 29 & $>-1.5 \mathrm{SD}$ on NPE & Petersen criteria ${ }^{1}$ & $\begin{array}{l}\text { NINCDS- } \\
\text { ADRA }^{3}\end{array}$ \\
\hline EDAR & $\begin{array}{l}\text { European } \\
\text { multicenter }\end{array}$ & 204 & $>-1.5 \mathrm{SD}$ on $\mathrm{NPE}$ & Petersen criteria ${ }^{1}$ & $\begin{array}{l}\text { NINCDS- } \\
\text { ADRA }^{3}\end{array}$ \\
\hline GAP & Spain & 40 & $>-1.5 \mathrm{SD}$ on $\mathrm{NPE}$ and $\mathrm{CDR}=0$ & Petersen criteria $^{1}$ & $\begin{array}{l}\text { NINCDS- } \\
\text { ADRA }^{3}\end{array}$ \\
\hline Gothenburg & Sweden & 95 & $>-1.5 \mathrm{SD}$ on $\mathrm{NPE}$ & Winblad criteria $^{2}$ & $\begin{array}{l}\text { NINCDS- } \\
\text { ADRA }^{3}\end{array}$ \\
\hline IDIBAPS & Spain & 120 & $>-1.5 \mathrm{SD}$ on $\mathrm{NPE}$ and $\mathrm{CDR}=0$ & Petersen $_{\text {criteria }}{ }^{1}$ & $\begin{array}{l}\text { NINCDS- } \\
\text { ADRA }^{3}\end{array}$ \\
\hline Lausanne & Switzerland & 40 & $>-1.5 \mathrm{SD}$ on $\mathrm{NPE}$ and $\mathrm{CDR}=0$ & $\begin{array}{l}\text { Winblad criteria }{ }^{2} \text {, } \\
\mathrm{CDR}=0.5\end{array}$ & $\begin{array}{l}\text { NINCDS- } \\
\text { ADRA }^{3}\end{array}$ \\
\hline Leuven & Belgium & 180 & $\begin{array}{l}>-1.5 \mathrm{SD} \text { on NPE, } \mathrm{CDR}=0 \text { and } \\
\mathrm{MMSE}>27\end{array}$ & Petersen $_{\text {criteria }}{ }^{1}$ & $\begin{array}{l}\text { NINCDS- } \\
\text { ADRA }^{3}\end{array}$ \\
\hline Pharmacog & $\begin{array}{l}\text { European } \\
\text { multicenter }\end{array}$ & 147 & $>-1.5 \mathrm{SD}$ on $\mathrm{NPE}$ & $\begin{array}{l}\text { Petersen } \text { criteria }^{1} \text {, } \\
\mathrm{CDR}=0.5\end{array}$ & $\begin{array}{l}\text { NINCDS- } \\
\text { ADRA }^{3}\end{array}$ \\
\hline Sant Pau & Spain & 45 & $>-1.5 \mathrm{SD}$ on $\mathrm{NPE}$ and GDS $<3$ & $\begin{array}{l}\text { Petersen } \text { criteria }^{1}, \\
\text { GDS }=3\end{array}$ & $\begin{array}{l}\text { NINCDS- } \\
\text { ADRA }^{3}\end{array}$ \\
\hline
\end{tabular}

$\mathrm{AD}=$ Alzheimer's disease, $\mathrm{CDR}=$ Clinical Dementia Rating, $\mathrm{CN}=$ Cognitively normal, DSM-IV = Diagnostic and Statistical Manual of Mental Disorders, GDS = global deterioration scale, MCI = Mild Cognitive Impairment, MMSE = Mini Mental State Examinations, NINCDS-ADRA = National Institute of Neurological and Communicative Disorders and Stroke - Alzheimer's Disease and Related Disorders Association; NPE = Neuropsychological Examination; ${ }^{1}$ Petersen RC: Mild Cognitive Impairment as a diagnostic entity. Journal of internal medicine 2004; ${ }^{2}$ Winblad B, Palmer K, Kivipelto, M. et al. Mild cognitive impairment - beyond controversies, towards a consensus: report of the International Working Group on Mild Cognitive Impairment. Journal of internal medicine. 2004; ${ }^{3}$ McKhann G., Drachman, D., Folstein, M., et al. Clinical diagnosis of Alzheimer's disease: report of the NINCDS-ADRDA Work Group under auspices of Department of Health and Human Services Task Force on Alzheimer's Disease. Neurology, 1984 
Chapter 3

Supplemental Table 2. Number of subjects per test by cognitive domain at baseline

\begin{tabular}{|c|c|c|c|}
\hline Cognitive Domain & Tests & $\mathrm{n}$ & Normative data used \\
\hline Global cognition & Mini Mental State Examination & 1216 & \\
\hline \multirow[t]{9}{*}{ Memory } & At least one test & 1197 & \\
\hline & AVLT & 613 & ${ }^{1,2}$ and local norms \\
\hline & Free and Cued Selective Reminding Test & 175 & $3-5$ \\
\hline & $\begin{array}{l}\text { Word list of CERAD neuropsychological } \\
\text { battery }\end{array}$ & 217 & ${ }^{6,7}$ and local norms \\
\hline & $\begin{array}{l}\text { Wechsler Memory Scale, subscales or whole } \\
\text { scale }\end{array}$ & 63 & 8 \\
\hline & RBANS, memory index & 49 & 9 \\
\hline & MMSE, memory items & 29 & $\begin{array}{l}\text { Cut-off }(<3) \text { to define } \\
\text { abnormality }\end{array}$ \\
\hline & HDS, recent memory items & 29 & $\begin{array}{l}\text { Cut-off }(<5) \text { to define } \\
\text { abnormality }\end{array}$ \\
\hline & RI-48 Test & 22 & Local norms \\
\hline \multirow[t]{7}{*}{ Language } & At least one test & 1182 & \\
\hline & 1-minute verbal fluency animals & 796 & 10 \\
\hline & Category fluency sum of three categories & 147 & 11 \\
\hline & Boston Naming Test & 113 & 12,13 \\
\hline & $\begin{array}{l}\text { 1-minute verbal fluency words starting with } \\
\text { 'F', 'A', 'S' }\end{array}$ & 90 & Local norms \\
\hline & 2-minute verbal fluency animals & 45 & Local norms \\
\hline & RBANS, language index & 31 & 9 \\
\hline \multirow[t]{6}{*}{ Attention } & At least one test & 1156 & \\
\hline & Trail Making Test, part A & 1035 & ${ }^{7,14}$ and local norms \\
\hline & Stroop, part 1 & 183 & ${ }^{15}$ and local norms \\
\hline & RBANS, concentration index & 47 & 9 \\
\hline & $\begin{array}{l}\text { Wechsler Memory Scale, working } \\
\text { memory/concentration }\end{array}$ & 40 & 8 \\
\hline & HDS, concentration index & 30 & $\begin{array}{l}\text { Cut-off }(<5) \text { to define } \\
\text { abnormality }\end{array}$ \\
\hline \multirow[t]{3}{*}{ Executive functioning } & At least one test & 970 & \\
\hline & Trail Making Test, part B & 957 & local \\
\hline & Stroop, part 3 & 15 & ${ }^{15}$ and local norms \\
\hline \multirow[t]{5}{*}{ Visuoconstruction } & At least one test & 719 & \\
\hline & Copy of Rey complex figure & 431 & ${ }^{4,16}$ and local norms \\
\hline & Copy CERAD figures & 207 & 7 \\
\hline & RBANS, visuoconstruction & 49 & 9 \\
\hline & HDS, drawing item & 32 & $\begin{array}{l}\text { Cut-off }(<5) \text { to define } \\
\text { abnormality }\end{array}$ \\
\hline
\end{tabular}

RAVLT $=$ Rey Auditory Verbal Learning Test, CERAD = Consortium to Establish a Registry for AD, HDS = Hasegawa Dementia Scale, MMSE = Mini Mental State Examination, RBANS = Repeatable Battery for the Assessment of Neuropsychological Status 1.Van der Elst W, van Boxtel MP, van Breukelen GJ, Jolles J. Rey's verbal 
learning test: normative data for 1855 healthy participants aged 24-81 years and the influence of age, sex, education, and mode of presentation. Journal of the International Neuropsychological Society : JINS. 2005;11(3):290-302; 2.Ivnik RJ, Malec JF, Tangalos EG, Petersen RC, Kokmen E, Kurland LT. The AuditoryVerbal Learning Test (AVLT): norms for ages 55 years and older. Psychological Assessment: A Journal of Consulting and Clinical Psychology. 1990;2(3):304; 3.Ivnik RJ, Smith GE, Lucas JA, Tangalos EG, Kokmen E, Petersen RC. Free and cued selective reminding test: MOANS norms. Journal of Clinical and Experimental Neuropsychology. 1997;19(5):676-691; 4.Peña-Casanova J, Gramunt-Fombuena N, Quiñones-Úbeda S, et al. Spanish multicenter normative studies (NEURONORMAProject): norms for the Rey-Osterrieth complex figure (copy and memory), and free and cued selective reminding test. Archives of Clinical Neuropsychology. 2009;24(4):371-393; 5.Dion M, Potvin O, Belleville S, et al. Normative data for the Rappel libre/Rappel indicé à 16 items (16-item Free and Cued Recall) in the elderly Quebec-French population. The Clinical neuropsychologist. 2015;28(sup1):1-19; 6.Welsh KA, Butters N, Mohs RC, et al. The Consortium to Establish a Registry for Alzheimer's Disease (CERAD). Part V. A normative study of the neuropsychological battery. Neurology. 1994;44(4):609-614; 7.Aebi C. Validierung der neuropsychologischen Testbatterie CERAD-NP: eine Multi-Center Studie, University_of_Basel; 2002; 8. Wechsler D. Weschler Memory Scale-3rd Edition. The Psychological Corporation New York, NY; 1997; 9.Pearson. Repeatable Battery for the Assessment of Neuropsychological Status (RBANS) ${ }^{\mathrm{m}}$. 2008; 10.Van der Elst W, Van Boxtel MP, Van Breukelen GJ, Jolles J. Normative data for the Animal, Profession and Letter M Naming verbal fluency tests for Dutch speaking participants and the effects of age, education, and sex. Journal of the International Neuropsychological Society: JINS. 2006;12(1):80-89; 11. Novelli G, Papagno C, Capitani E, Laiacona M. Tre test clinici di ricerca e produzione lessicale. Taratura su sogetti normali. Archivio di psicologia, neurologia e psichiatria. 1986; 12.Marien P, Mampaey E, Vervaet A, Saerens J, De Deyn PP. Normative data for the Boston Naming Test in native Dutchspeaking Belgian elderly. Brain and language. 1998;65(3):447-467; 13. Peña-Casanova J, Quiñones-Úbeda S, Gramunt-Fombuena N, et al. Spanish Multicenter Normative Studies (NEURONORMA Project): norms for Boston naming test and token test. Archives of Clinical Neuropsychology. 2009;24(4):343-354; 14. Norms for Stroop Color Word test, Trail making test, and Story recall of Rivermead Behavioural Memory Test. 2003; 15.Van der Elst W, Van Boxtel MP, Van Breukelen GJ, Jolles J. The Stroop color-word test: influence of age, sex, and education; and normative data for a large sample across the adult age range. Assessment. 2006;13(1):62-79; 16.Caffarra P, Vezzadini G, Dieci F, Zonato F, Venneri A. Rey-Osterrieth complex figure: normative values in an Italian population sample. Neurological Sciences. 2002;22(6):443-447 


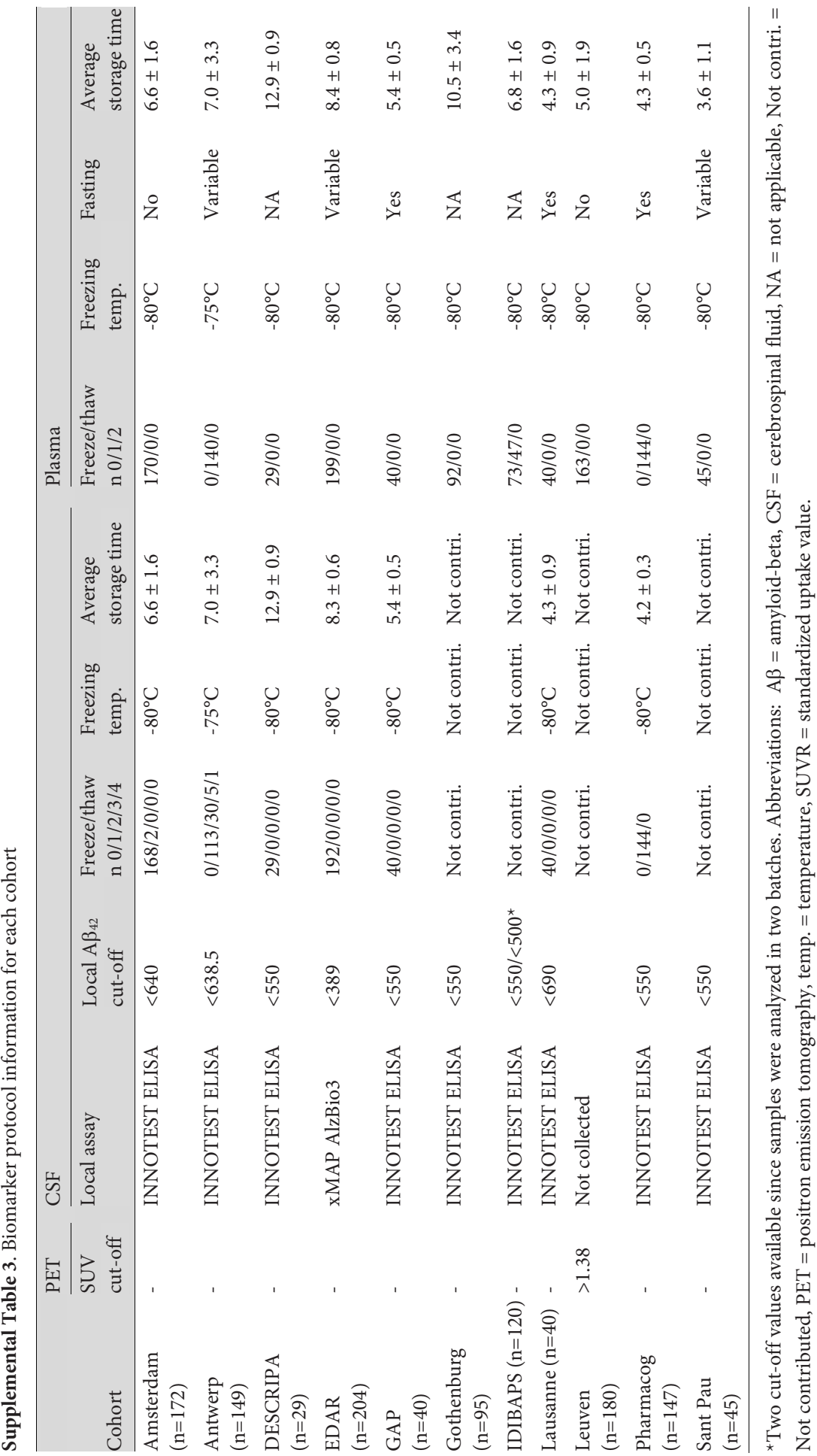






\section{Part}

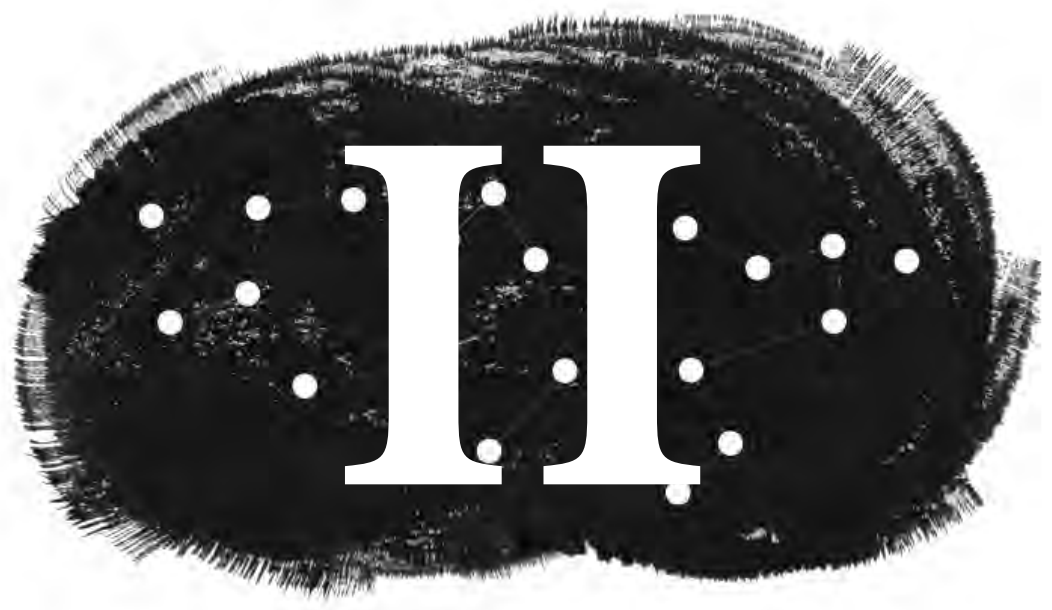

\section{Vascular contributions to Alzheimer's disease pathology}




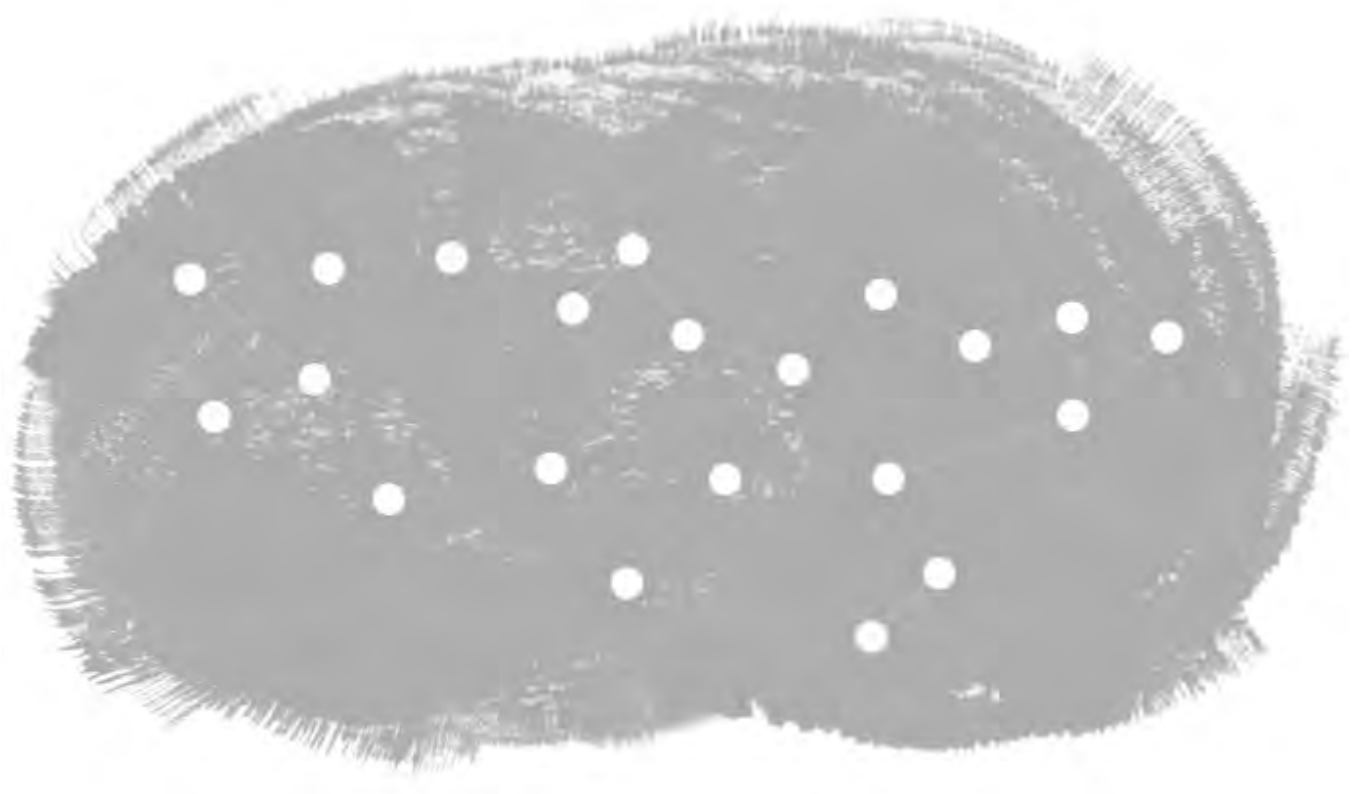




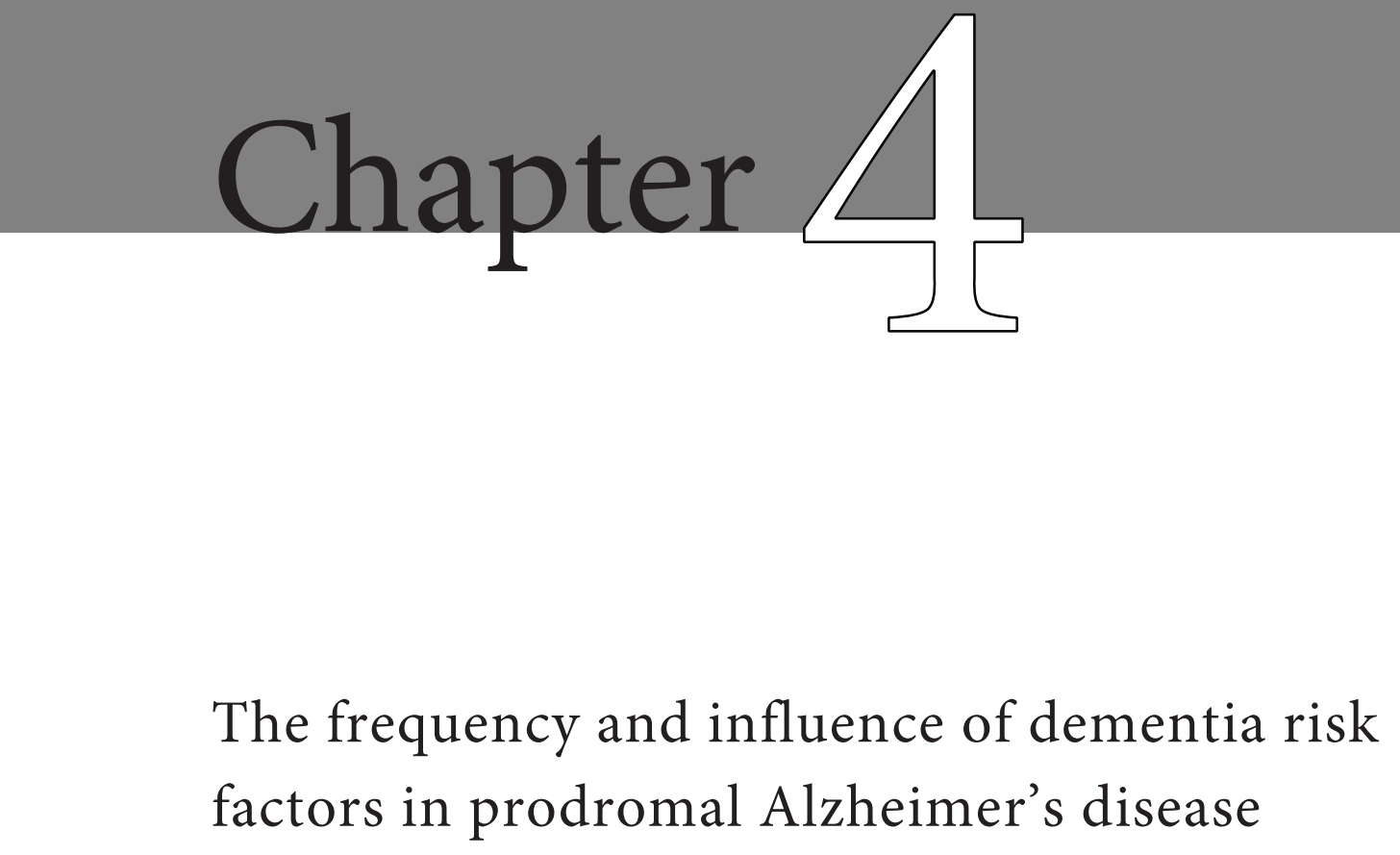

Neurobiology of Aging 2017; 56, 33-40

Isabelle Bos, Stephanie J. Vos, Lutz Frölich, Johannes Kornhuber, Jens Wiltfang, Wolfgang Maier, Oliver Peters, Eckhart Rüther, Sebastiaan Engelborghs, Ellis Niemantsverdriet, Ellen Elisa De Roeck, Magda Tsolaki, Yvonne Freund-Levi, Peter Johannsen, Rik Vandenberghe, Alberto Lleó, Daniel Alcolea, Giovanni B. Frisoni, Samantha Galluzzi, Flavio Nobili, Silvia Morbelli, Alexander Drzezga, Mira Didic, Bart N. van Berckel, Eric Salmon, Christine Bastin, Solene Dauby, Isabel Santana, Inês Baldeiras, Alexandre de Mendonça, Dina Silva, Anders Wallin, Arto Nordlund, Preciosa M. Coloma, Angelika Wientzek, Myriam Alexander, Gerald P. Novak, Mark Forrest Gordon, the Alzheimer's Disease Neuroimaging Initiative, Åsa K. Wallin, Harald Hampel, Hilkka Soininen, Sanna-Kaisa Herukka, Philip Scheltens, Frans Verhey and Pieter Jelle Visser. 


\begin{abstract}
We investigated whether dementia risk factors were associated with prodromal Alzheimer's disease (AD) according to the International Working Group-2 and National Institute of Aging-Alzheimer's Association criteria, and with cognitive decline. 1394 subjects from with Mild Cognitive Impairment (MCI) from 14 different studies, were classified according to the research criteria, based on cognitive performance and biomarkers. We compared the frequency of ten risk factors between the subgroups and used Coxregression to examine the effect of risk factors on cognitive decline. In individuals with a high-AD-likelihood, depression, obesity and hypercholesterolemia occurred less frequent, compared to those without a low-AD-likelihood. Alcohol increased the risk of cognitive decline, regardless of $\mathrm{AD}$-pathology. These results suggest that traditional risk factors for $\mathrm{MCI}$ and $\mathrm{AD}$, are not associated with $\mathrm{AD}$ pathology, in subjects with MCI. Besides alcohol, none of the risk factors increased the risk of cognitive decline in subjects with prodromal AD. Future studies should validate these findings and determine whether risk factors might be of influence at an earlier stage (i.e. preclinical) of AD.
\end{abstract}




\section{INTRODUCTION}

Various risk factors have been associated with an increased risk for Alzheimer's disease (AD). ${ }^{1,2}$ Recently, research criteria have been proposed to identify AD in subjects with mild cognitive impairment (MCI) by their biomarker status, referred to as prodromal AD by international working group- 2 (IWG-2) ${ }^{3}$ and $\mathrm{MCI}$ due to $\mathrm{AD}$ by the National Institute of Aging-Alzheimer Association (NIA-AA). ${ }^{4}$ It remains uncertain whether risk factors are associated with prodromal $\mathrm{AD} / \mathrm{MCI}$ due to $\mathrm{AD}$, and whether they influence the rate of cognitive decline. This information could improve early diagnosis and lead to new targets for secondary prevention strategies.

Among the best-validated risk factors for $\mathrm{AD}$ are atherosclerosis, depression, diabetes mellitus, hypercholesterolemia, hypertension, lacunar infarcts, stroke, obesity, smoking, and alcohol consumption. ${ }^{1,2,5}$ Diabetes mellitus, depression, hypertension, stroke and cardiovascular diseases have also been associated with an increased risk of progressing from cognitively normal to MCI. ${ }^{6,7}$ Moreover, an association with cognitive decline has been found in both cognitively normal and MCI subjects. ${ }^{8,9}$ Therefore, we hypothesize that risk factors will occur more frequently in individuals with prodromal $\mathrm{AD} / \mathrm{MCI}$ due to $\mathrm{AD}$. We also expect that risk factors will increase the risk of progression to dementia.

We aim to investigate the frequency of several risk factors in individuals with prodromal $\mathrm{AD} / \mathrm{MCI}$ due to $\mathrm{AD}$, classified according to the IWG-2 and NIA-AA criteria, relative to subjects who do not meet these criteria. Secondly, we aim to examine whether risk factors influence the rate of cognitive decline.

\section{METHODS}

\section{Subjects}

Subjects were recruited from five multicenter memory-clinic based studies: DESCRIPA, ${ }^{10}$ German Dementia Competence Network (DCN), ${ }^{11}$ EDAR (www.edarstudy.eu), the European Alzheimer's Disease Consortium (EADC)-PET study ${ }^{12}$ and American Alzheimer's Disease Neuroimaging Initiative (ADNI-1) study ${ }^{13}$; and nine centers of the EADC and/or European Medical Information Framework (EMIF)-AD: Amsterdam, ${ }^{14}$ Antwerp ${ }^{15}$ Barcelona, ${ }^{16}$ Brescia, ${ }^{17}$ Coimbra, ${ }^{18}$ Gothenburg, ${ }^{19}$ Kuopio, ${ }^{20}$ Liège,${ }^{21}$ and Lisbon. ${ }^{22}$ For subjects who participated in more than one study, we used data from the study with the longest follow-up.

Inclusion criteria consisted of baseline diagnosis of MCI according to the criteria of Petersen, ${ }^{23}$ and at least one of the following biomarkers available at baseline: amyloid-beta 
(A $\beta$ ) 1-42 and tau (total tau and/or phosphorylated tau) in CSF, hippocampal volume on magnetic resonance imaging (MRI) or cerebral glucose metabolism on [18F] FDG-PET of the brain. Moreover, baseline data had to be available on at least one of the selected risk factors, as well as information on educational level and at least one clinical follow-up assessment. Exclusion criteria were diagnosis of dementia at baseline.

\section{Clinical assessment}

The clinical assessment is described in detail by Vos et al. ${ }^{24}$ In short, clinical assessment was performed at each site according to local routine protocol. Cognitive impairment was defined as Z-score $<-1.5 \mathrm{SD}$ on at least one neuropsychological test, which could be a memory or non-memory test.

\section{Outcome at follow-up}

Cognitive decline was defined as progression to dementia according to the Diagnostic and Statistical Manual of Mental Disorders, ${ }^{25}$ or a decline on the Mini-Mental State Examination (MMSE) of at least 3 points at follow-up. We used a combination of these two measures, as for a subgroup $(\mathrm{n}=17)$ no clinical diagnosis at follow-up was available. For sub analyses, diagnosis of $\mathrm{AD}$-type dementia at follow-up was made according to the $\mathrm{Na}$ tional Institute of Neurological and Communicative Disorders and Stroke - Alzheimer's Disease and Related Disorders Association criteria (NINCDS-ADRDA). ${ }^{26}$

The medical ethics committee at each site approved the study. All subjects provided informed consent.

\section{Biomarker assessment}

Biomarker assessment was performed according to the routine protocol at each site and center-specific cut-offs were used to define abnormality, as described elsewhere. ${ }^{24}$ Examination of medial temporal lobe atrophy on MRI and cerebral glucose metabolism on FDG-PET were performed through visual assessment.

\section{Subject classification}

Subjects were classified as having prodromal AD according to the IWG-2 criteria using CSF A $\beta 1-42$ and tau biomarkers (Table 1). The NIA-AA criteria distinguish between six groups that indicate the likelihood that $\mathrm{MCI}$ is due to $\mathrm{AD}$, based on combinations of amyloid and neuronal injury markers. We used CSF A $\beta 1-42$ as amyloid marker and CSF total tau, CSF phosphorylated tau, cerebral glucose metabolism on FDG-PET, hippocampal volume or medial temporal lobe atrophy on MRI as neuronal injury markers (Table 1). 


\section{Risk factors}

We assessed the following risk factors at baseline: atherosclerotic disease, depression, diabetes, hypercholesterolemia, hypertension, lacunar infarct, obesity, stroke, current smoking, and current alcohol use. Not all risk factors were available for each subject. Supplemental Table 1 provides an overview of the available risk factors for each center. The risk factor definitions are described by center in Supplemental Table 2. For all risk factors occurrence in medical history was used as a standard. For some risk factors, we used additional definitions based on rating scales, physical measurements or medication use, based on availability (Supplemental Table 2).

\section{Statistical analyses}

Baseline differences between the biomarker profile groups were analyzed using ANOVA for continuous variables and Chi-Square test for categorical variables. The relation of risk factors with prodromal $\mathrm{AD} / \mathrm{MCI}$ due to $\mathrm{AD}$ was tested with logistic regression (IWG-2 criteria) or multinomial regression (NIA-AA criteria). Cox proportional hazards models were used to test the effect of each risk factor on the rate of cognitive decline in the total sample, and for the IWG-2 and NIA-AA biomarker subgroups. All analyses were adjusted for age, sex, education and center. Statistical analyses were performed using SPSS version 22.0 with the significance level set at $\mathrm{p}<0.05$. We corrected for multiple comparisons, using the false discovery rate (FDR) adjustment ${ }^{27}$, taking into account the testing of ten risk factors. In tables, we reported uncorrected p-values and we indicated which associations were significant after correction for multiple comparisons in tables and the text.

\section{RESULTS}

\section{Subject characteristics}

We included 1394 individuals (mean age $=69.7$, SD 8.3; 51\% female). Seven hundred and fifty-eight subjects had data available on both amyloid and neuronal injury markers, while 636 subjects only had data on a neuronal injury marker (medial temporal lobe atrophy $\mathrm{n}=528$, FDG-PET $\mathrm{n}=108$ ). Five hundred and eighty individuals (42\%) showed cognitive decline after an average follow-up time of 2.3 (SD 1.2) years. Table 2 shows the characteristics of the subjects classified according to the IWG-2 and the NIA-AA criteria. 
Table 1. Classification of subjects according to IWG-2 and NIA-AA criteria

\begin{tabular}{lll}
\hline IWG-2 groups & $\begin{array}{l}\text { Amyloid marker: } \\
\text { CSF A } \beta \text { 1-42 }\end{array}$ & $\begin{array}{l}\text { Neuronal injury marker: } \\
\text { CSF t-tau or p-tau }\end{array}$ \\
\hline No prodromal AD & Abnormal & Normal \\
& Normal & Abnormal \\
& Normal & Normal \\
Prodromal AD & Abnormal & Abnormal \\
& Amyloid Marker & Neuronal injury markers \\
\hline NIA-AA groups & & Neuronal injury markers: \\
\hline Low-AD-likelihood & Amyloid marker: & CSF t-tau or p-tau / MTA on MRI / \\
High-AD-likelihood & CSF A $\beta$ 1-42 & FDG-PET \\
IAP & Normal & All normal \\
SNAP & Abnormal & At least one abnormal \\
Intermediate-AD-likelihood & Abnormal & All normal \\
Inconclusive/uninformative & Normal & At least one abnormal \\
\hline
\end{tabular}

$\mathrm{A} \beta=$ amyloid-beta, $\mathrm{AD}=$ Alzheimer's disease, $\mathrm{CSF}=$ cerebrospinal fluid, FDG-PET = fluorodeoxyglucose-positron emission tomography, IAP = Isolated Amyloid Pathology, IWG = International Working Group, MRI = magnetic resonance imaging, MTA = Medial Temporal Lobe Atrophy, NIA-AA = National Institute of AgingAlzheimer's Association, $\mathrm{p}$-tau $=$ phosphorylated tau, SNAP = non-Alzheimer Pathophysiology, $\mathrm{t}$-tau $=$ total tau.

\section{Analyses in subjects with both amyloid and neuronal injury markers}

Based on the IWG-2 criteria, 302 subjects (40\%) were classified as prodromal AD. Individuals with prodromal $\mathrm{AD}$ were older $(\mathrm{p}<0.001)$, showed a lower score on the MMSE at follow-up ( $\mathrm{p}<0.001)$ and were more likely to progress to AD-type dementia at follow-up $(\mathrm{p}<0.001)$ compared to subjects without prodromal AD. According to the NIA-AA criteria, 142 individuals (10\%) were classified in the low-AD-likelihood group, 356 (26\%) in the high-AD-likelihood group, 54 (4\%) in the Isolated Amyloid Pathology (IAP) group, and 206 (15\%) in the Suspected Non-AD Pathophysiology (SNAP) group. Subjects in the high-AD-likelihood group were older and most likely to progress to $\mathrm{AD}$-type dementia, compared to all other groups (Table 2).

\section{Frequency of risk factors}

Table 3 shows the frequency of $\mathrm{AD}$ risk factors for the NIA-AA groups with both amyloid and neuronal injury data. Compared to the low-AD-likelihood group, subjects in the high-AD-likelihood group had a lower frequency of depression ( $17 \%$ vs. $46 \%, \mathrm{p}=0.004$, FDR $\mathrm{p}=0.020$ ), obesity ( $8 \%$ vs. $21 \%, \mathrm{p}=0.004$, FDR $\mathrm{p}=0.020$ ) and hypercholesterolemia ( $27 \%$ vs. $43 \%, p=0.009$, FDR $p=0.030$ ). No differences were found between the groups for the other risk factors (Table 3 ). 


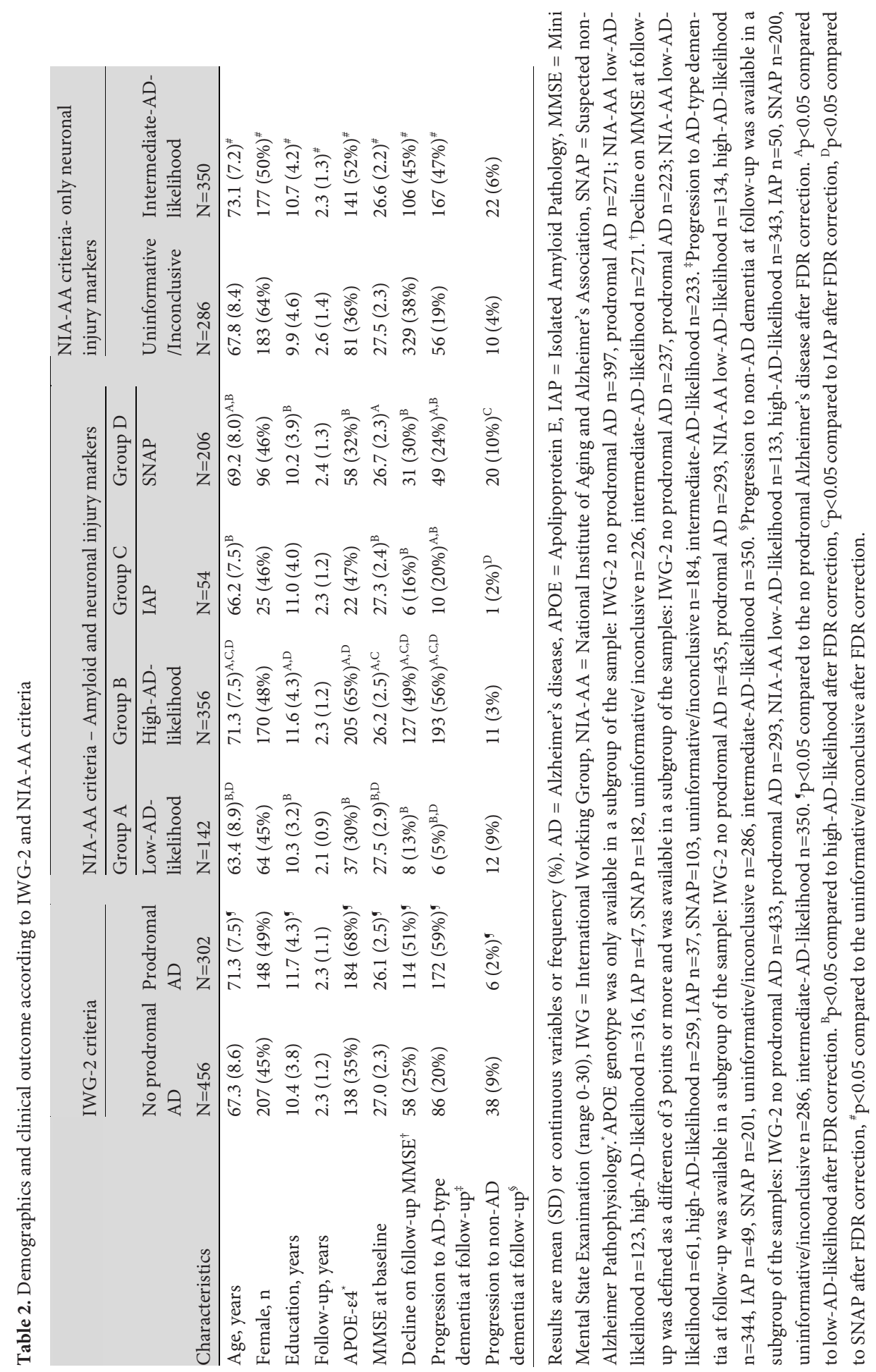


Supplemental Table 3 shows the frequency of risk factors for the groups according to the IWG- 2 criteria. Subjects with prodromal AD, had less depression ( $16 \%$ vs. $34 \%$, $p=0.009$, FDR $\mathrm{p}=0.045$ ) and obesity ( $8 \%$ vs. $17 \%, \mathrm{p}=0.007$, FDR $\mathrm{p}=0.045$ ) compared to the group without prodromal AD (Supplemental Table 3).

Table 3: Frequency of risk factors for NIA-AA groups

\begin{tabular}{llllll}
\hline & $\begin{array}{l}\text { Low-AD- } \\
\text { likelihood }\end{array}$ & $\begin{array}{l}\text { High-AD- } \\
\text { likelihood }\end{array}$ & IAP & SNAP & $\begin{array}{l}\text { p-value } \\
\text { Low vs. High }\end{array}$ \\
\cline { 2 - 5 } Risk factors & $\mathrm{N}=142$ & $\mathrm{~N}=356$ & $\mathrm{~N}=54$ & $\mathrm{~N}=206$ & \\
\hline Atherosclerotic disease $(\mathrm{n}=1002)$ & $4 \%$ & $10 \%$ & $5 \%$ & $9 \%$ & 0.277 \\
Depression (n=1129) & $46 \%$ & $17 \%^{*}$ & $27 \%$ & $29 \%$ & 0.004 \\
Diabetes $(\mathrm{n}=914)$ & $8 \%$ & $9 \%$ & $14 \%$ & $15 \%$ & 0.960 \\
Hypercholesterolemia $(\mathrm{n}=1001)$ & $43 \%$ & $27 \%^{*}$ & $38 \%$ & $38 \%$ & 0.009 \\
Hypertension $(\mathrm{n}=1346)$ & $50 \%$ & $47 \%$ & $54 \%$ & $47 \%$ & 0.038 \\
Lacunar infarct $(\mathrm{n}=497)$ & $29 \%$ & $23 \%$ & $18 \%$ & $30 \%$ & 0.133 \\
Stroke $(\mathrm{n}=1013)$ & $3 \%$ & $4 \%$ & $6 \%$ & $5 \%$ & 0.787 \\
Obesity $(\mathrm{n}=993)$ & $21 \%$ & $8 \% *$ & $8 \%$ & $18 \%$ & 0.004 \\
Smoking $(\mathrm{n}=1195)$ & $53 \%$ & $36 \%$ & $40 \%$ & $42 \%$ & 0.076 \\
Alcohol use $(\mathrm{n}=973)$ & $42 \%$ & $50 \%$ & $50 \%$ & $42 \%$ & 0.352 \\
\hline
\end{tabular}

$\mathrm{AD}=$ Alzheimer's disease, IAP = Isolated Amyloid pathology, SNAP = Suspected non-Alzheimer Pathophysiology. Comparisons were corrected for baseline age, gender, years of education and center. ${ }^{*} \mathrm{p}<0.05$ after FDR correction.

\section{Effect of risk factors on cognitive decline}

In the total group of subjects with both amyloid and neuronal injury markers, alcohol use was associated with a higher risk of cognitive decline $(\mathrm{HR}=1.5, \mathrm{p}=0.003$, FDR $\mathrm{p}=0.030$, Table 4). There were no significant interactions between risk factors and NIA-AA group classification, indicating that the effect of risk factors was similar for all groups. Using the IWG-2 classification, the effects of depression, hypercholesterolemia, and smoking were different between the two groups, but these differences were no longer statistical significant after adjusting for multiple testing (Table 4).

\section{Analyses in subjects with only neuronal injury markers}

Table 2 shows the characteristics of the 258 (21\%) subjects classified as uninformative/inconclusive and the 350 (25\%) included in the intermediate-AD-likelihood group according to the NIA-AA criteria. The subjects in the intermediate-AD-likelihood differed on all characteristics from the uninformative/inconclusive group. 


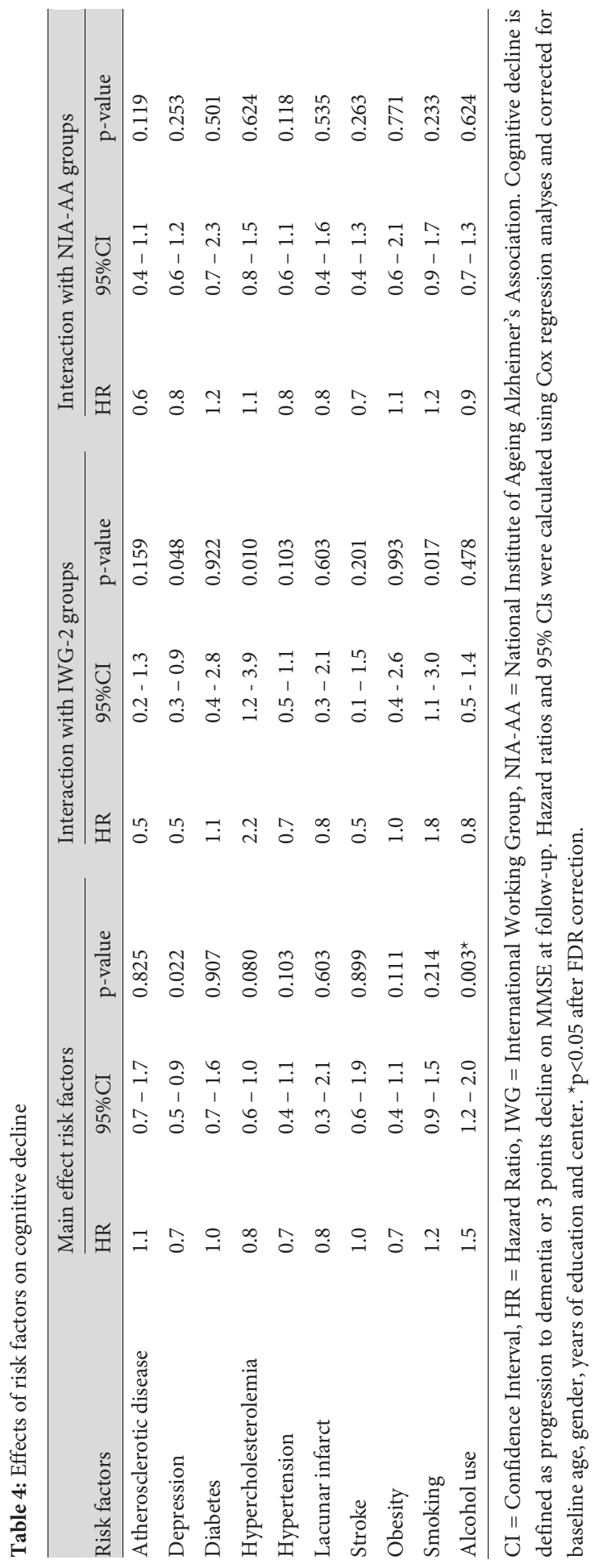


The frequency of risk factors for the subjects who had only neuronal injury markers available is described in Supplemental Table 4. In the intermediate-AD-likelihood-group lacunar infarcts occurred more frequently (40\%), compared to the uninformative/inconclusive group $(16 \%, \mathrm{p}<0.001)$. There were no differences for the other risk factors (Supplemental Table 4).

In the subjects with only neuronal injury markers available, none of the risk factors increased the risk of cognitive decline. Also, there was no difference between the two NIAAA groups in the rate of cognitive decline (Supplemental Table 5).

\section{Post-hoc analyses - progression to AD-type dementia}

When we repeated the analyses with only progression to AD-type dementia as an outcome in subjects with both amyloid and neuronal injury markers available $(n=725)$, alcohol use was no longer associated with an increased risk of progression ( $\mathrm{HR}=1.3,95 \% \mathrm{CI}$ : $0.9-1.8, \mathrm{p}=0.164)$.

Since the ADNI cohort excluded subjects with depressive symptoms (GDS $>6$ ), we repeated the analyses concerning depression without ADNI subjects. This did not influence the results.

\section{DISCUSSION}

We examined the frequency of vascular, psychiatric, and lifestyle risk factors in prodromal $\mathrm{AD} / \mathrm{MCI}$ due to $\mathrm{AD}$, and the influence of these factors on cognitive decline, in subjects with MCI. We found that, in our sample, risk factors were not associated with prodromal $\mathrm{AD} / \mathrm{MCI}$ due to $\mathrm{AD}$. Only alcohol increased the risk of cognitive decline, regardless of AD-pathology.

\section{Frequency of risk factors}

We found a lower frequency of depression and obesity and a trend for hypercholesterolemia, in the prodromal $\mathrm{AD}$ and high-AD-likelihood group, compared to the group without prodromal $\mathrm{AD}$ and the low-AD-likelihood group. The lower frequency of obesity in the prodromal $\mathrm{AD}$ and high-AD-likelihood groups, is in line with a previous study demonstrating that (involuntary) weight loss is associated with $\mathrm{AD}$, prior to onset of dementia. ${ }^{28}$ This suggests that being overweight might be a risk factor for dementia in midlife, whereas a reduction in weight could actually be indication for dementia, later in life. This inverse relationship between bodyweight and dementia, has also been previously described in a recent study. ${ }^{29}$ In general, the higher frequency of risk factors in the group 
without prodromal $\mathrm{AD}$ and in the low-AD-likelihood group suggests that, most likely, these subjects had cognitive impairment due to another cause than AD such as depression, vascular disorders or non-AD type dementia. ${ }^{30,31}$ This stresses the heterogeneity of MCI, and the importance of biomarkers to make this diagnosis more specific. Particularly in the light of predicting clinical progression and selecting individuals for clinical trials.

The low frequency of hypercholesterolemia in the high-AD-likelihood could be explained by the age of onset of the disease, as a meta-analysis on population-based studies showed that the negative effect of hypercholesterolemia on development of $\mathrm{AD}$, only applies to individuals who develop hypercholesterolemia at midlife, whereas a decreased risk for AD was found for individuals who develop hypercholesterolemia later in life. ${ }^{32}$

\section{Influence of risk factors on cognitive decline}

Alcohol consumption was associated with an increased risk of cognitive decline, independent of AD-pathology. Although this finding is in line with several previous studies ${ }^{5,33}$ that identified alcohol as a risk factor for cognitive decline, other studies have reported a protective or no relation to alcohol consumption with incident AD. ${ }^{34,35}$ These conflicting results could be explained by differences in study population (MCI versus cognitively normal), definitions of alcohol consumption (dichotomous versus categories based on the amount of alcohol use) and the type of alcohol. When conversion to AD-type dementia was used as outcome instead of conversion to dementia or a decline on the MMSE, we found that the effect of alcohol was no longer significant. This suggests that alcohol consumption mainly has an effect on progression to non-AD types of dementia or cognitive decline in general.

\section{IWG-2 vs. NIA-AA criteria}

The results on frequency of risk factors were comparable for the IWG-2 and NIA-AA criteria. Also, when comparing the effect of risk factors on cognitive decline we found similar outcomes when using the two sets of criteria. This shows that although the IWG2 criteria only classify neuronal injury based on tau in CSF and the NIA-AA criteria also include other neuronal injury markers, this did not influence the results. Although the outcomes were similar for the two sets of criteria, the NIA-AA criteria provided more insight into which specific biomarker profile was associated with a higher frequency of a certain risk factor, which could be useful to give a more refined diagnosis and prognosis of early $\mathrm{AD}$ and age-related comorbidities. 


\section{Comparison to epidemiological studies}

While population based studies typically find that depression and cardiovascular risk factors increase the risk of progression to dementia, ${ }^{1,5,36}$ we found that MCI subjects with a high likelihood for AD had a lower frequency of depression, obesity and hypercholesterolemia compared to subjects with a low-AD-likelihood. As we did not include cognitively normal subjects in our study, we cannot rule out the possibility that these risk factors would be more common in subjects with prodromal $\mathrm{AD}$, compared to cognitively normal subjects. To explore this possibility, we compared the frequencies of risk factors in the low and high-AD likelihood groups, to the frequency of these risk factors as reported in meta-analyses and population based cohort studies (Supplemental Table 6). The frequency of hypercholesterolemia and obesity in the general population was similar to that in the low-AD-likelihood group, but higher compared to the high-AD-likelihood group, showing that the frequency of these factors is probably decreased in the high-AD-likelihood group. The frequency of depression in the general population was similar to that in the high-AD-likelihood group, but lower than in low-AD-likelihood group. This is in line with our previous suggestion that subjects in the low-AD-likelihood group might have MCI due to depression. Clearly, there are methodological differences in the inclusion of subjects, definition and method of ascertainment of risk factors and age range between the current study and the population based studies. Studies that directly compare frequency of risk factors in prodromal AD to cognitively normal subjects are needed to further clarify this.

\section{Strengths and limitations of the study}

Strengths of the study are the large sample size, the broad spectrum of assessed risk factors and longitudinal data on clinical outcome. There are also limitations to this study that should be mentioned. Some of the biomarker subgroups were small and some risk factors were only available in a subgroup or had a low frequency, which limited statistical power. The data used in this study was contributed by different centers and data was not collected using the same protocol. This might have led to variability, although it does reflect current clinical practice. Furthermore, the use of indirect measures (e.g. medical history) of risk factors could have introduced heterogeneity in classification. We were unable to study the potential interactions between risk factors, as not all centers contributed data on all risk factors. We had only limited data available on medication use, which did not allow us to control for this. Also, we could not correct for the duration and the severity of a risk factor, as we had no information on this. Although the mean follow-up was 2.3 years, some individuals likely would have shown cognitive decline at a later stage. Since these findings are based on clinical research populations, they may not be generalizable to other settings. 


\section{Conclusion}

In summary, we found that dementia risk factors were not associated with prodromal $\mathrm{AD} / \mathrm{MCI}$ due to $\mathrm{AD}$ in subjects with $\mathrm{MCI}$, and only alcohol increased the risk of cognitive decline, regardless of underlying pathology. Although our findings should be validated in future studies, they could have implications for clinical practice, future scientific studies, as well as for selection of individuals for participation in clinical trials. Different risk factor profiles in subjects with MCI could be related to distinct etiologies of cognitive dysfunction, and therefore may have different prognostic values. Management of alcohol habits could possibly lessen or prevent further cognitive decline. Future studies should focus on the role of risk factors in even earlier stages of $\mathrm{AD}$ (e.g. preclinical $\mathrm{AD}$ ), examine longitudinal biomarkers values, and consider the duration and severity of risk factors. Also, cooccurrence of risk factors and possible synergistic effects on biomarkers should be a topic for future research as we were unable to study this in the current sample. 


\section{REFERENCES}

1. Breteler MM. Vascular risk factors for Alzheimer's disease: an epidemiologic perspective. Neurobiology of aging. 2000;21(2):153-160.

2. de Bruijn RF, Ikram MA. Cardiovascular risk factors and future risk of Alzheimer's disease. BMC Med. 2014;12:130.

3. Dubois B, Feldman HH, Jacova C, et al. Advancing research diagnostic criteria for Alzheimer's disease: the IWG-2 criteria. The Lancet Neurology. 2014;13(6):614-629.

4. Albert MS, DeKosky ST, Dickson D, et al. The diagnosis of mild cognitive impairment due to Alzheimer's disease: recommendations from the National Institute on Aging-Alzheimer's Association workgroups on diagnostic guidelines for Alzheimer's disease. Alzheimer's \& dementia : the journal of the Alzheimer's Association. 2011;7(3):270-279.

5. Deckers K, van Boxtel MP, Schiepers OJ, et al. Target risk factors for dementia prevention: a systematic review and Delphi consensus study on the evidence from observational studies. International journal of geriatric psychiatry. 2015;30(3):234-246.

6. Pankratz VS, Roberts RO, Mielke MM, et al. Predicting the risk of mild cognitive impairment in the Mayo Clinic Study of Aging. Neurology. 2015;84(14):1433-1442.

7. Roberts RO, Cha RH, Mielke MM, et al. Risk and protective factors for cognitive impairment in persons aged 85 years and older. Neurology. 2015;84(18):1854-1861.

8. Jefferson AL, Hohman TJ, Liu D, et al. Adverse vascular risk is related to cognitive decline in older adults. Journal of Alzheimer's disease : JAD. 2015;44(4):1361-1373.

9. Kaffashian S, Dugravot A, Elbaz A, et al. Predicting cognitive decline: a dementia risk score vs. the Framingham vascular risk scores. Neurology. 2013;80(14):1300-1306.

10. Visser PJ, Verhey FR, Boada M, et al. Development of screening guidelines and clinical criteria for predementia Alzheimer's disease. The DESCRIPA Study. Neuroepidemiology. 2008;30(4):254-265.

11. Kornhuber J, Schmidtke K, Frolich L, et al. Early and differential diagnosis of dementia and mild cognitive impairment: design and cohort baseline characteristics of the German Dementia Competence Network. Dementia and geriatric cognitive disorders. 2009;27(5):404-417.

12. Morbelli S, Drzezga A, Perneczky R, et al. Resting metabolic connectivity in prodromal Alzheimer's disease. A European Alzheimer Disease Consortium (EADC) project. Neurobiology of aging. 2012;33(11):2533-2550.

13. Mueller SG, Weiner MW, Thal LJ, et al. Ways toward an early diagnosis in Alzheimer's disease: the Alzheimer's Disease Neuroimaging Initiative (ADNI). Alzheimer's \& dementia : the journal of the Alzheimer's Association. 2005;1(1):55-66.

14. van der Flier WM, Pijnenburg YA, Prins N, et al. Optimizing patient care and research: the Amsterdam Dementia Cohort. Journal of Alzheimer's disease : JAD. 2014;41(1):313-327.

15. Somers C. SH, Goossens J., Niemantsverdriet E., Luyckx J., De Roeck N., De Roeck E., De Vil B., Cras P., Martin J.J., De Deyn PP., Bjerke M., Engelborghs S. A decade of cerebrospinal fluid biomarkers for Alzheimer's disease in Belgium. In press.

16. Alcolea D, Carmona-Iragui M, Suarez-Calvet M, et al. Relationship between beta-Secretase, inflammation and core cerebrospinal fluid biomarkers for Alzheimer's disease. Journal of Alzheimer's disease : JAD. 2014;42(1):157-167.

17. Frisoni GB, Prestia A, Zanetti O, et al. Markers of Alzheimer's disease in a population attending a memory clinic. Alzheimer's \& dementia : the journal of the Alzheimer's Association. 2009;5(4):307-317.

18. Baldeiras I, Santana I, Proenca MT, et al. Peripheral oxidative damage in mild cognitive impairment and mild Alzheimer's disease. Journal of Alzheimer's disease : JAD. 2008;15(1):117-128. 
19. Wallin A, Nordlund A, Jonsson M, et al. Alzheimer's disease--subcortical vascular disease spectrum in a hospital-based setting: Overview of results from the Gothenburg MCI and dementia studies. J Cereb Blood Flow Metab. 2016;36(1):95-113.

20. Seppala TT, Koivisto AM, Hartikainen P, Helisalmi S, Soininen H, Herukka SK. Longitudinal changes of CSF biomarkers in Alzheimer's disease. Journal of Alzheimer's disease : JAD. 2011;25(4):583-594.

21. Bastin C, Kerrouche N, Lekeu F, et al. Controlled memory processes in questionable Alzheimer's disease: A view from neuroimaging research. Journal of Alzheimer's Disease. 2010;20(2):547-560.

22. Maroco J, Silva D, Rodrigues A, Guerreiro M, Santana I, de Mendonca A. Data mining methods in the prediction of Dementia: A real-data comparison of the accuracy, sensitivity and specificity of linear discriminant analysis, logistic regression, neural networks, support vector machines, classification trees and random forests. BMC research notes. 2011;4:299.

23. Petersen RC. Mild cognitive impairment as a diagnostic entity. Journal of internal medicine. 2004;256(3):183-194.

24. Vos SJ, Verhey F, Frolich L, et al. Prevalence and prognosis of Alzheimer's disease at the mild cognitive impairment stage. Brain : a journal of neurology. 2015;138(Pt 5):1327-1338.

25. APA. Diagnostic and Statistical Manual of Mental Disorders: DSM-IV. Washington, DC: American Psychiatric Association; 1994.

26. McKhann G, Drachman D, Folstein M, Katzman R, Price D, Stadlan EM. Clinical diagnosis of Alzheimer's disease: report of the NINCDS-ADRDA Work Group under the auspices of Department of Health and Human Services Task Force on Alzheimer's Disease. Neurology. 1984;34(7):939-944.

27. Benjamini Y, Hochberg Y. Controlling the false discovery rate: a practical and powerful approach to multiple testing. Journal of the royal statistical society Series B (Methodological). 1995:289-300.

28. Johnson DK, Wilkins CH, Morris JC. Accelerated weight loss may precede diagnosis in Alzheimer disease. Archives of neurology. 2006;63(9):1312-1317.

29. Batty GD, Galobardes B, Starr JM, Jeffreys M, Davey Smith G, Russ TC. Examining if being overweight really confers protection against dementia: Sixty-four year follow-up of participants in the Glasgow University alumni cohort study. Journal of negative results in biomedicine. 2016;15(1):19.

30. DeCarli C. Mild cognitive impairment: prevalence, prognosis, aetiology, and treatment. The Lancet Neurology. 2003;2(1):15-21.

31. Gorelick PB, Scuteri A, Black SE, et al. Vascular contributions to cognitive impairment and dementia: a statement for healthcare professionals from the american heart association/american stroke association. Stroke; a journal of cerebral circulation. 2011;42(9):2672-2713.

32. Anstey KJ, Lipnicki DM, Low LF. Cholesterol as a risk factor for dementia and cognitive decline: a systematic review of prospective studies with meta-analysis. Am J Geriatr Psychiatry. 2008;16(5):343-354.

33. Jauhar S, Marshall EJ, Smith ID. Alcohol and cognitive impairment. Advances in psychiatric treatment. 2014;20(5):304-313.

34. Anstey KJ, Mack HA, Cherbuin N. Alcohol consumption as a risk factor for dementia and cognitive decline: meta-analysis of prospective studies. Am J Geriatr Psychiatry. 2009;17(7):542-555.

35. Ruitenberg A, van Swieten JC, Witteman JC, et al. Alcohol consumption and risk of dementia: the Rotterdam Study. Lancet. 2002;359(9303):281-286.

36. Helzner EP, Luchsinger JA, Scarmeas N, et al. Contribution of vascular risk factors to the progression in Alzheimer disease. Archives of neurology. 2009;66(3):343-348. 


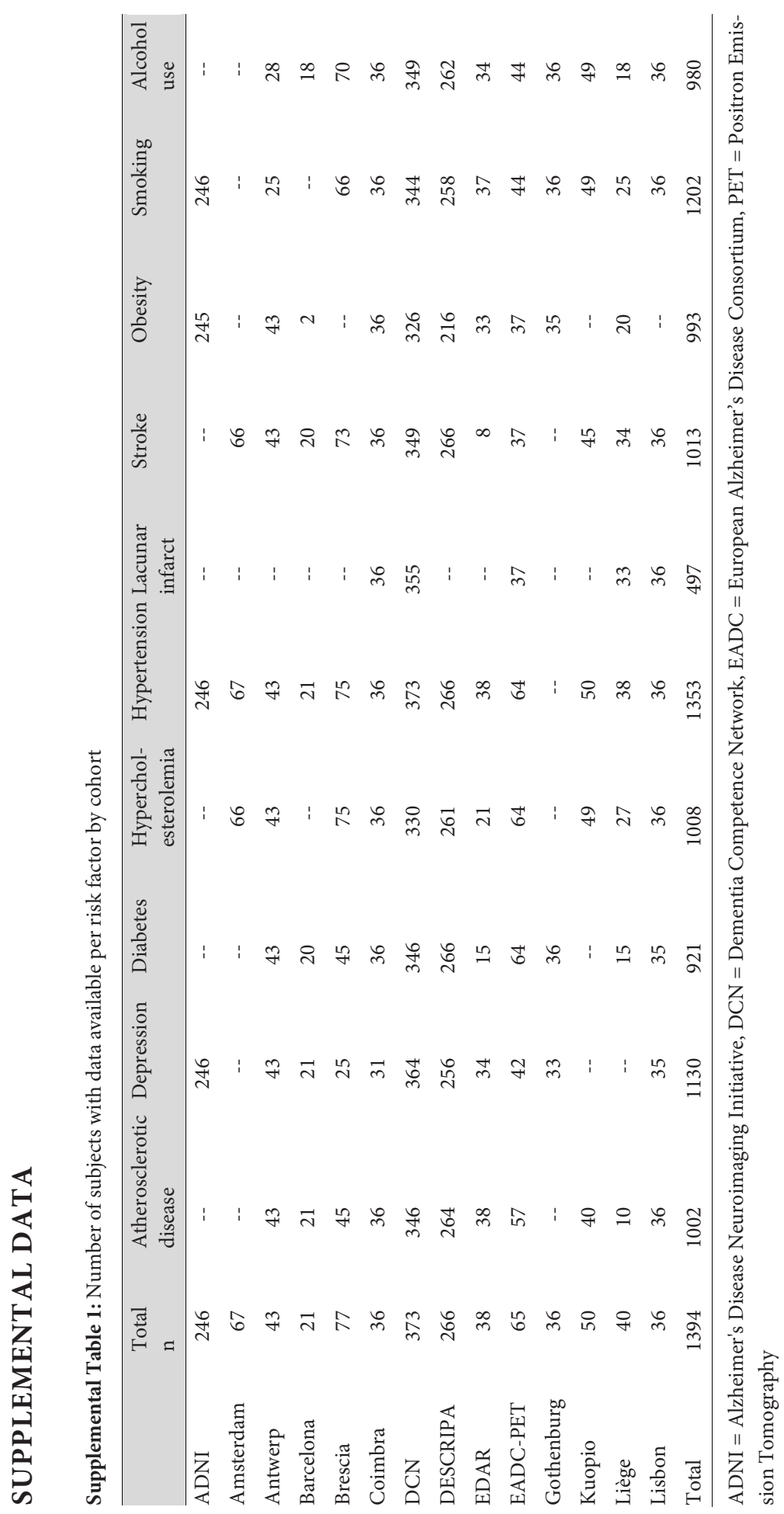




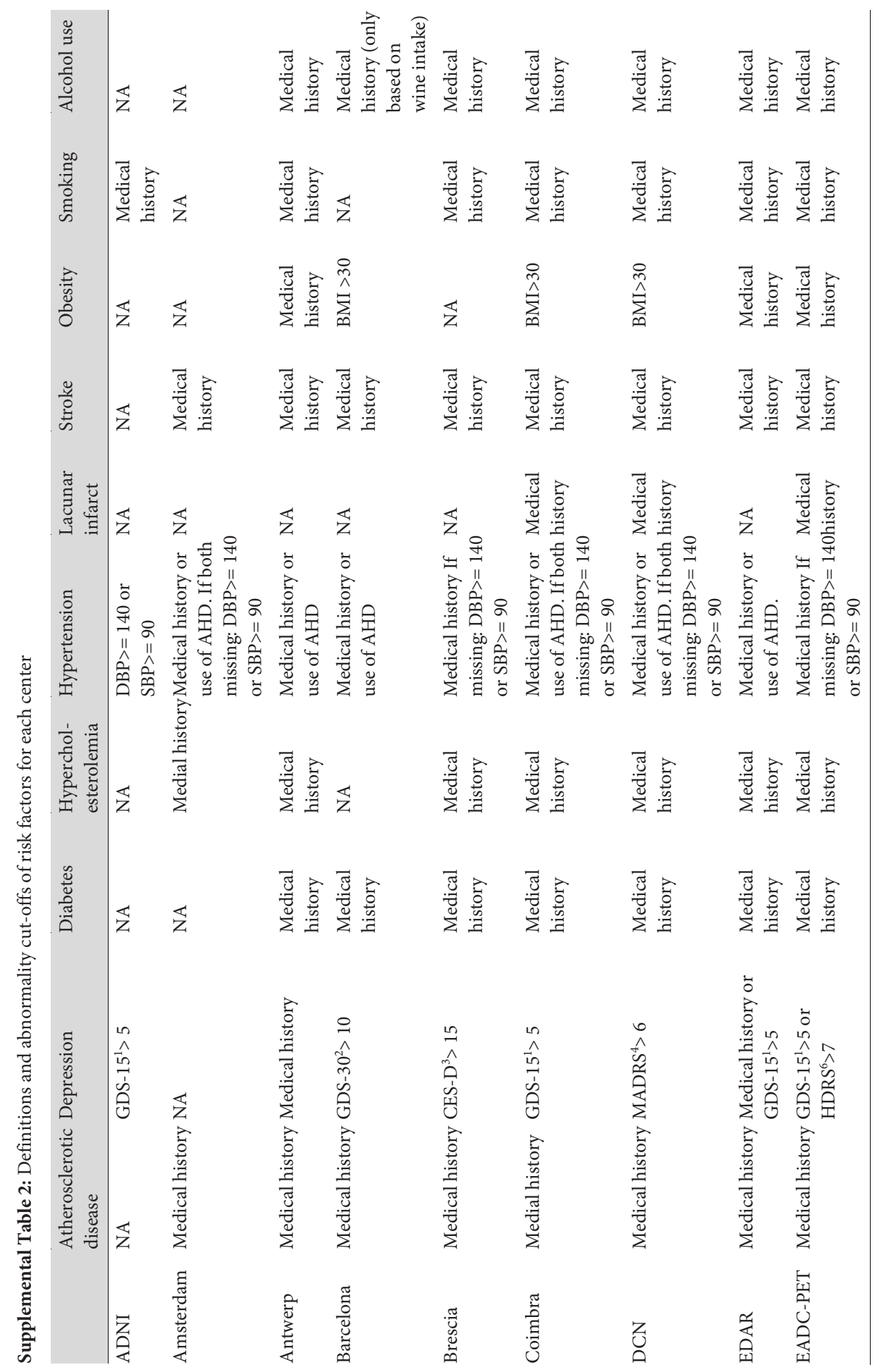




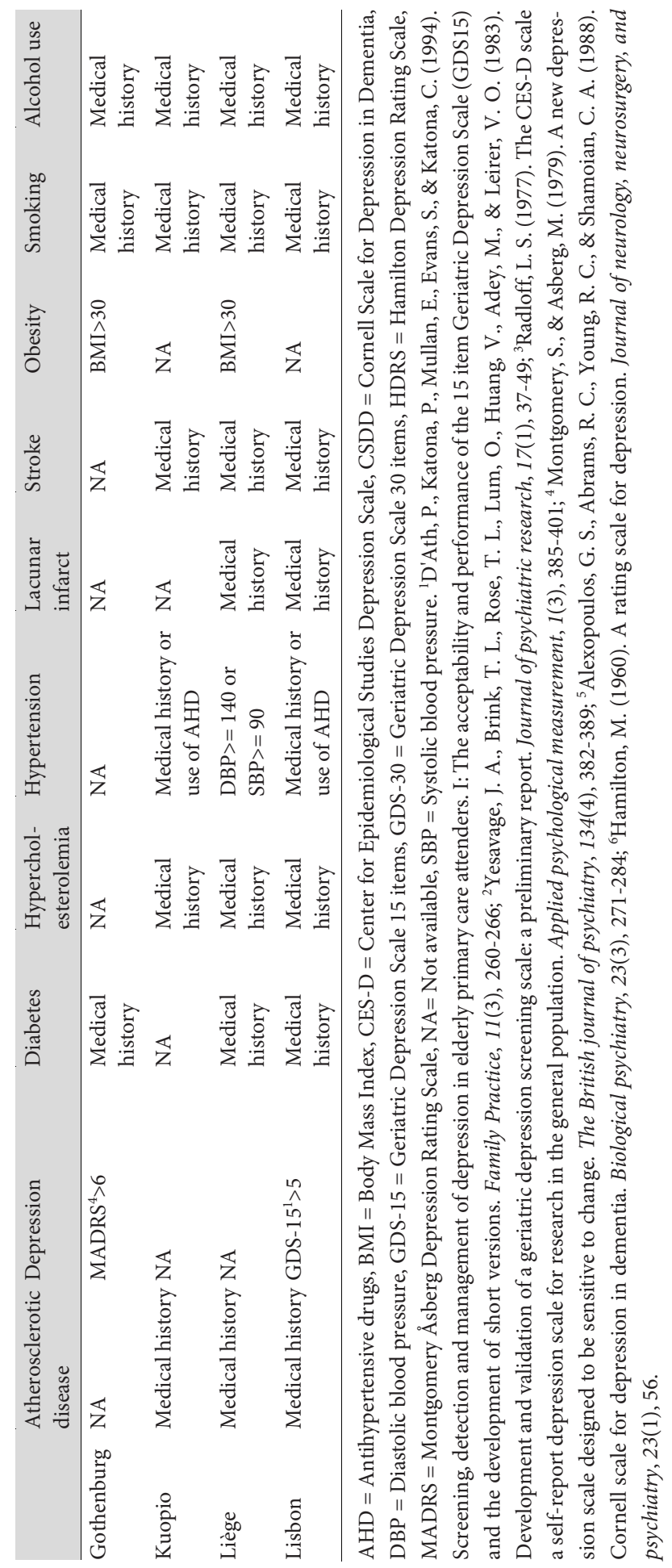


Supplemental Table 3: Frequency of risk factors for IWG-2 groups

\begin{tabular}{llll}
\hline & $\begin{array}{l}\text { No prodromal AD } \\
\text { Risk factors }\end{array}$ & $\begin{array}{l}\text { Prodromal AD } \\
\mathrm{n}=459\end{array}$ & p-value \\
\hline Atherosclerotic disease $(\mathrm{n}=1002)$ & $7 \%$ & $10 \%$ & 0.495 \\
Depression $(\mathrm{n}=1129)$ & $34 \%$ & $16 \%^{*}$ & 0.009 \\
Diabetes $(\mathrm{n}=914)$ & $12 \%$ & $7 \%$ & 0.101 \\
Hypercholesterolemia $(\mathrm{n}=1001)$ & $38 \%$ & $28 \%$ & 0.035 \\
Hypertension $(\mathrm{n}=1346)$ & $49 \%$ & $46 \%$ & 0.116 \\
Lacunar infarct $(\mathrm{n}=497)$ & $28 \%$ & $23 \%$ & 0.372 \\
Stroke $(\mathrm{n}=1013)$ & $4 \%$ & $4 \%$ & 0.804 \\
Obesity $(\mathrm{n}=993)$ & $17 \%$ & $8 \% *$ & 0.007 \\
Smoking $(\mathrm{n}=1195)$ & $44 \%$ & $37 \%$ & 0.258 \\
Alcohol use $(\mathrm{n}=973)$ & $44 \%$ & $50 \%$ & 0.404 \\
\hline
\end{tabular}

$\mathrm{AD}=$ Alzheimer's disease. Comparisons were corrected for baseline age, gender, years of education and center. ${ }^{*} \mathrm{p}<0.05$ after FDR correction.

Supplemental Table 4: Frequency of risk factors for NIA-AA criteria intermediate-AD-likelihood and uninformative/inconclusive group

\begin{tabular}{lll}
\hline & $\begin{array}{l}\text { Uninformative/ } \\
\text { Inconclusive }\end{array}$ & Intermediate-AD-likelihood \\
\cline { 2 - 3 } Risk factors & $\mathrm{N}=286$ & $\mathrm{~N}=350$ \\
\hline Atherosclerotic disease $(\mathrm{n}=1002)$ & $9 \%$ & $14 \%$ \\
Depression $(\mathrm{n}=1129)$ & $35 \%$ & $24 \%$ \\
Diabetes $(\mathrm{n}=914)$ & $14 \%$ & $12 \%$ \\
Hypercholesterolemia $(\mathrm{n}=1001)$ & $51 \%$ & $43 \%$ \\
Hypertension $(\mathrm{n}=1346)$ & $48 \%$ & $50 \%$ \\
Lacunar infarct $(\mathrm{n}=497)$ & $16 \%$ & $40 \% *$ \\
Stroke $(\mathrm{n}=1013)$ & $4 \%$ & $4 \%$ \\
Obesity $(\mathrm{n}=993)$ & $13 \%$ & $12 \%$ \\
Smoking $(\mathrm{n}=1195)$ & $32 \%$ & $36 \%$ \\
Alcohol use $(\mathrm{n}=973)$ & $40 \%$ & $46 \%$ \\
\hline
\end{tabular}

All comparisons are corrected for baseline age, gender, years of education and center. ${ }^{*} \mathrm{p}<0.05$ compared to the uninformative/inconclusive group. 
Supplemental Table 5: Effects of risk factors on cognitive decline in intermediate-AD-likelihood and uninformative/inconclusive groups

\begin{tabular}{|c|c|c|c|c|c|c|}
\hline \multirow[b]{2}{*}{ Risk factors } & \multicolumn{3}{|c|}{ Main effects risk factors } & \multicolumn{3}{|c|}{ Interaction with two NIA-AA groups } \\
\hline & HR & $95 \% \mathrm{CI}$ & p-value & HR & $95 \% \mathrm{CI}$ & p-value \\
\hline Atherosclerotic disease & 1.0 & $0.6-1.6$ & 0.989 & 1.1 & $0.4-2.9$ & 0.854 \\
\hline Depression & 0.7 & $0.5-0.9$ & 0.135 & 1.3 & $0.6-2.7$ & 0.785 \\
\hline Diabetes & 0.9 & $0.6-1.4$ & 0.704 & 0.8 & $0.3-2.1$ & 0.816 \\
\hline Hypercholesterolemia & 0.8 & $0.6-1.0$ & 0.135 & 1.3 & $0.7-2.3$ & 0.785 \\
\hline Hypertension & 0.8 & $0.6-1.0$ & 0.135 & 0.9 & $0.5-1.4$ & 0.816 \\
\hline Lacunar infarct & 1.6 & $1.0-2.4$ & 0.135 & 0.6 & $0.2-1.6$ & 0.785 \\
\hline Stroke & 1.0 & $0.5-2.0$ & 0.989 & 0.2 & $0.1-0.9$ & 0.310 \\
\hline Obesity & 0.7 & $0.5-1.2$ & 0.418 & 1.1 & $0.4-3.1$ & 0.854 \\
\hline Smoking & 1.1 & $0.8-1.4$ & 0.704 & 0.7 & $0.4-1.3$ & 0.785 \\
\hline Alcohol use & 0.9 & $0.7-1.2$ & 0.704 & 1.8 & $1.0-3.5$ & 0.310 \\
\hline
\end{tabular}

$\mathrm{CI}=$ Confidence Interval, HR = Hazard Ratio, NIA-AA = National Institute of Ageing Alzheimer's Association. Cognitive decline is defined as progression to dementia or 3 points decline on MMSE at follow-up. Hazard ratios and 95\% CIs were calculated using Cox regression analyses and corrected for baseline age, gender, years of education and center.

Supplemental Table 6: Frequency of risk factors in IWG-2 groups and in the general population

\begin{tabular}{|c|c|c|c|c|c|}
\hline Risk factors & $\begin{array}{l}\text { Low-AD- } \\
\text { likelihood }\end{array}$ & $\begin{array}{l}\text { High-AD- } \\
\text { likelihood }\end{array}$ & $\begin{array}{l}\text { General } \\
\text { population }\end{array}$ & $\begin{array}{l}\text { Age } \\
\text { group }\end{array}$ & Reference \\
\hline Atherosclerotic disease $(n=1002)$ & $4 \%$ & $10 \%$ & $28 \%$ & $70-79$ & Vos et al., $2017^{1}$ \\
\hline Depression $(\mathrm{n}=1129)$ & $46 \%$ & $17 \%^{*}$ & $17 \%$ & $\geq 75$ & Luppa et al., $2012^{2}$ \\
\hline Diabetes $(\mathrm{n}=914)$ & $8 \%$ & $9 \%$ & $11 \%$ & $70-79$ & Vos et al., $2017^{1}$ \\
\hline Hypercholesterolemia $(n=1001)$ & $43 \%$ & $27 \% *$ & $41 \%$ & $\geq 65$ & Félix-Redondo et al., $2013^{3}$ \\
\hline Hypertension $(n=1346)$ & $50 \%$ & $47 \%$ & $75 \%$ & $70-79$ & Vos et al., $2017^{1}$ \\
\hline Lacunar infarct $(\mathrm{n}=497)$ & $29 \%$ & $23 \%$ & $23 \%$ & $\geq 65$ & Longstreth et al., $1998^{4}$ \\
\hline Stroke $(n=1013)$ & $3 \%$ & $4 \%$ & $12 \%$ & $\geq 65$ & Di Carlo et al., $2000^{5}$ \\
\hline Obesity (n=993) & $21 \%$ & $8 \% *$ & $17 \%$ & $\geq 50$ & Andreyeva et al., $2007^{6}$ \\
\hline Smoking $(\mathrm{n}=1195)$ & $53 \%$ & $36 \%$ & $29 \%$ & $70-79$ & Vos et al., $2017^{1}$ \\
\hline Alcohol use $(n=973)$ & $42 \%$ & $50 \%$ & $63 \%$ & $70-79$ & Vos et al., $2017^{1}$ \\
\hline
\end{tabular}

${ }^{*} \mathrm{p}<0.05$ compared to the low-AD-likelihood group. ${ }^{1}$ Vos, S.J.B., van Boxtel, M.P.J., Schiepers, O.J.G., et al. (2017) Modifiable risk factors for prevention of dementia in midlife, late life and the oldest-old: the LIBRA Index. Journal of Alzheimer's disease, 58(2), 537-547. ${ }^{2}$ Luppa, M., Sikorski, C., Luck, et al. (2012). Age- and gender-specific prevalence of depression in latest-life - Systematic review and meta-analysis. Journal of Affective Disorders, 136, 212-221. ${ }^{3}$ Félix-Redondo, F.J., Grau, M., Fernández-Bergés, D. (2013). Cholesterol and Cardiovascular Disease in the Elderly. Facts and Gaps. Aging and Disease, 4(3), 154-169. ${ }^{4}$ Longstreth, W.T.jr., Bernick, C., Manolio, T.A., et al. (1998). Lacunar infarcts defined by magnetic resonance imaging of 3660 elderly people: the Cardiovascular Health Study. Archives of Neurology, 55(9), 1217-1225. ${ }^{5}$ Di Carlo, A., Launer, L.J., Breteler, M.M., et al. (2000). Frequency of stroke in Europe: A collaborative study of population-based cohorts. ILSA Working Group and the Neurologic Diseases in the Elderly Research Group. Italian Longitudinal Study on Aging. Neurology, 54, S28-33. ${ }^{6}$ Andreyeva T, Michaud PC, van Soest A. Obesity and health in Europeans aged 50 years and older. Public health. 2007;121:497-509. 


\section{Supplemental Text 1: Methods ADNI Study.}

Data used in the preparation of this article were partially obtained from the Alzheimer's Disease Neuroimaging Initiative (ADNI) database (adni.loni.usc.edu). The ADNI was launched in 2003 by the National Institute on Aging (NIA), the National Institute of Biomedical Imaging and Bioengineering (NIBIB), the Food and Drug Administration (FDA), private pharmaceutical companies and non-profit organizations, as a $\$ 60$ million, 5-year public-private partnership. The primary goal of ADNI has been to test whether serial magnetic resonance imaging (MRI), positron emission tomography (PET), other biological markers, and clinical and neuropsychological assessment can be combined to measure the progression of mild cognitive impairment (MCI) and early Alzheimer's disease $(\mathrm{AD})$. Determination of sensitive and specific markers of very early $\mathrm{AD}$ progression is intended to aid researchers and clinicians to develop new treatments and monitor their effectiveness, as well as lessen the time and cost of clinical trials. The Principal Investigator of this initiative is Michael W. Weiner, MD, VA Medical Center and University of California-San Francisco. ADNI is the result of efforts of many co-investigators from a broad range of academic institutions and private corporations, and subjects have been recruited from over 50 sites across the U.S. and Canada. The initial goal of ADNI was to recruit 800 subjects but ADNI has been followed by ADNIGO and ADNI-2. To date these three protocols have recruited over 1500 adults, ages 55 to 90 , to participate in the research, consisting of cognitively normal older individuals, people with early or late $\mathrm{MCI}$, and people with early $\mathrm{AD}$. The follow up duration of each group is specified in the protocols for ADNI-1, ADNI-2 and ADNI-GO. Subjects originally recruited for ADNI-1 and ADNIGO had the option to be followed in ADNI-2. For up-to-date information, see www.adni-info.org. 


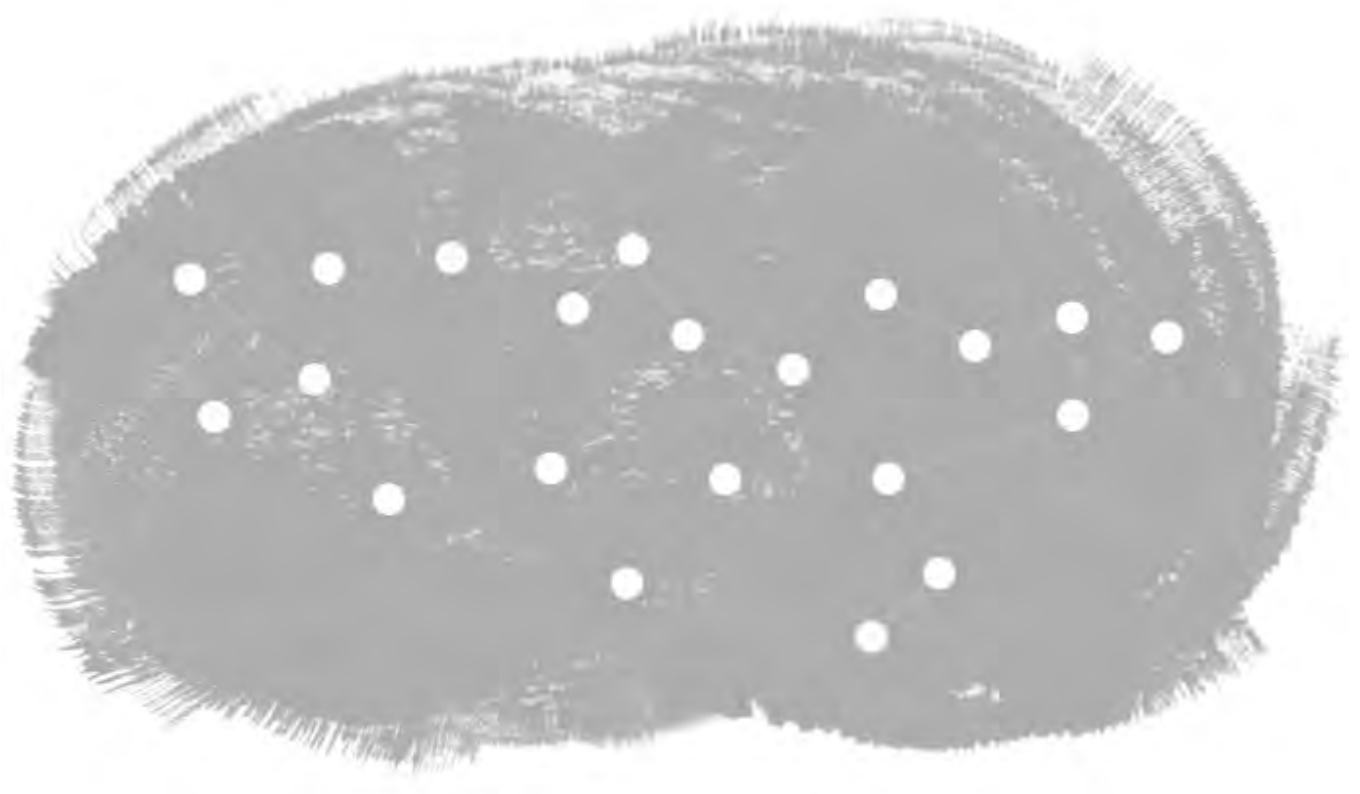




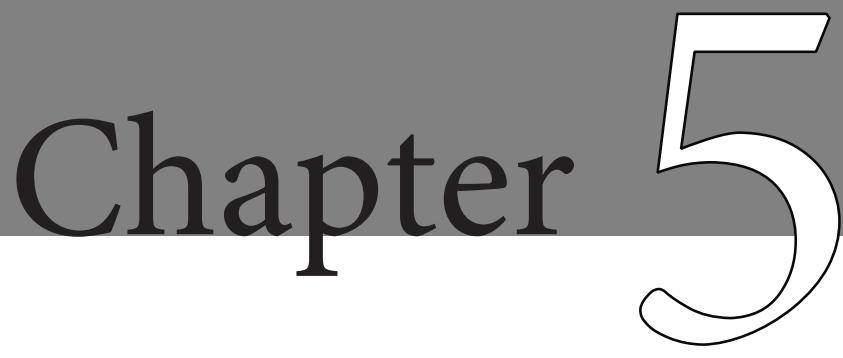

Vascular risk factors are associated with cerebrospinal fluid biomarker and cognitive changes in preclinical Alzheimer's disease

Submitted

Isabelle Bos, Stephanie J.B. Vos, Suzanne Schindler, Jason Hassenstab, Chengjie Xiong, Elizabeth Grant, Frans Verhey, John C. Morris, Pieter Jelle Visser and Anne M. Fagan 


\section{Chapter 5}

\section{ABSTRACT}

Background: Several lifestyle factors and co-morbid disorders have been associated with an increased risk for Alzheimer's disease (AD). Whether such conditions also increase the risk of $\mathrm{AD}$ pathology in the asymptomatic, "preclinical" stage remains uncertain. Aim of this study was to investigate whether lifestyle factors and co-morbid disorders are associated with longitudinal AD biomarker changes in cerebrospinal fluid (CSF) and with cognitive decline in cognitively normal individuals.

Methods: We selected 433 participants (mean age: 68.3, 53\% female) from the longitudinal studies at the Knight Alzheimer's Disease Research Center at Washington University in St. Louis. All were cognitively normal at baseline and had data available on cognition, $A P O E$ genotyping and CSF levels of amyloid- $\beta_{42}\left(A \beta_{42}\right)$, total tau ( $t$-tau) and phosphorylated $\operatorname{tau}_{181}$ ( $\mathrm{p}$-tau). Four biomarker groups were created based on baseline $\mathrm{A} \beta$ status (A $+/-)$ and tau status defined by either abnormal t-tau or p-tau $(\mathrm{T}+/-)$. We compared the frequency of 12 risk factors and the Framingham Risk score (FRS) among groups, and tested the influence of risk factors on change in biomarker concentrations and cognition over time.

Results: Obesity was less common in $\mathrm{T}+$ groups and cardiovascular disorders were more common in the $\mathrm{A}+\mathrm{T}+$ group, compared to the $\mathrm{A}-\mathrm{T}$ - group. In the total group, lower body mass index (BMI), hypertension and high FRS were associated with a faster increase in tau concentrations over time. For hypertension and the FRS this effect was only present in A+ individuals. Hypercholesterolemia was associated with a slower rate of cognitive decline in the total group and a higher FRS with a faster rate of decline in the $\mathrm{A}+\mathrm{T}+$ group.

Conclusions: Hypertension and a high FRS may enhance neurodegeneration in individuals who already have amyloid pathology. This suggests that treatment of these factors may be considered as secondary prevention in preclinical AD. 


\section{INTRODUCTION}

Several lifestyle factors and co-morbid disorders have been associated with an increased risk of cognitive decline and Alzheimer's disease (AD) dementia. ${ }^{1-4}$ However, little is known about the role of these conditions in $\mathrm{AD}$ prior to the onset of cognitive symptoms (i.e. preclinical $\mathrm{AD}$ ), as associated cross-sectional biomarker studies are inconclusive, ${ }^{5-7}$ and studies addressing this in relation to longitudinal biomarker changes are limited., ${ }^{8,9}$ Examining associations between $\mathrm{AD}$ risk factors, longitudinal AD biomarkers and cognitive decline in cognitively normal older individuals will increase understanding of underlying disease mechanisms and may also help to identify potential targets for primary and secondary $\mathrm{AD}$ prevention strategies.

Despite the relatively small number of studies and the inconsistency in findings, several vascular risk factors appear to be associated with $\mathrm{AD}$ biomarkers and cognition in the pre-dementia stages. ${ }^{5-7,10,11}$ The influence of these vascular factors seems strongest before the onset of clinical symptoms (i.e. in the preclinical stage) ${ }^{6,12}$ and may result in faster cognitive decline when occurring simultaneously. ${ }^{9,10}$ However, it remains uncertain which factors have the strongest influence on AD pathology, and whether they affect both amyloid deposition and neurodegeneration. In addition, more clarity regarding the influence of individual risk factors on cognitive decline in preclinical AD may improve prognostic accuracy. Hence, studying a broad spectrum of $\mathrm{AD}$ risk factors in relation to changes in $\mathrm{AD}$ biomarkers and cognition over time in cognitively normal individuals, could provide novel insights about early disease mechanisms and cognitive trajectories.

Our first aim was to investigate the influence of 12 established risk factors on AD pathology and longitudinal development of AD pathology over time in a large cohort of cognitively normal individuals. As a secondary aim, we examined whether these risk factors influenced the relationship between $\mathrm{AD}$ biomarkers and cognitive decline.

\section{METHODS}

\section{Participants}

Participants were selected from the longitudinal studies of memory and aging at the Knight Alzheimer's Disease Research Center (ADRC) at Washington University School of Medicine in St. Louis. Details about study design and protocols have been published previously. ${ }^{13}$ Participants in the current study $(n=433)$ were selected based on the following criteria: 1) Clinical Dementia Rating $(\mathrm{CDR})^{14}$ of 0 at baseline, indicating normal cognition; 2) CSF collection within one year of clinical/cognitive assessment; and 3) completed at least one annual clinical follow-up assessment. For the current study, the clinical 
visit closest to the first CSF collection was considered baseline. The study was approved by the Human Research Protection Office and Washington University, and all participants provided written informed consent.

\section{Risk factors}

The following risk factors were assessed: hypertension, hypercholesterolemia, diabetes mellitus, vitamin B12 deficiency, thyroid disease, depression, current smoking, alcohol abuse, transient ischemic attack (TIA), stroke, obesity and cardiovascular disorders. Risk factors were based on self or proxy-reported information during baseline assessment. Using a structured questionnaire, risk factors were coded as absent, recent/active, or remote/inactive. For the current study, both the recent/active and the remote/inactive categories were coded as 1 , and compared to the absent category (coded as 0 ). Definitions of all risk factors are listed in Supplemental Table 1. All risk factors were defined as present when reported as medical history or based on available additional information (e.g. use of medication, physical measures, etc.). In addition to the assessment of individual risk factors, a composite Framingham risk score (FRS) was calculated based on plasma total and high-density lipoprotein (HDL) cholesterol, systolic blood pressure, smoking status and medical history of diabetes, as described in detail elsewhere. ${ }^{15}$

\section{Cognitive measures}

Cognitive assessment was performed annually and was conducted by trained psychometricians who were blinded to the participant's CDR or diagnosis. The neuropsychological tests that were assessed for this study were the Free and Cued Selective Reminding Test free immediate recall portion (FCSRT-free) ${ }^{16}$; the Trail making Test parts A \& B (trails A and $B)^{17}$; the Animal Naming task ${ }^{18}$ and the Digit Symbol task from the Wechsler Adult Intelligence Scale-R. ${ }^{19}$ A cognitive composite average $\mathrm{z}$-score was created from all available cognitive measures using the baseline mean and standard deviation of all participants included in this study. ${ }^{20}$ The Mini-Mental State Examination (MMSE) ${ }^{21}$ was completed as part of the clinical assessment.

\section{CSF collection and analyses}

CSF samples (20-30 mL) were collected for all 433 individuals at baseline, and for 184 individuals also at one $(n=107)$, two $(n=54)$ or three $(n=23)$ follow-up visits. All samples were collected at $8 \mathrm{AM}$ by gravity drip lumbar puncture (LP), following overnight fasting. The CSF was gently inverted to disrupt potential gradient effects, shortly centrifuged at low speed to pellet any cellular debris, and aliquoted $(0.5 \mathrm{~mL})$ into polypropylene tubes prior to freezing at $-80^{\circ} \mathrm{C}$, as described in detail elsewhere. ${ }^{22}$ Amyloid- $\beta$ 1-42 $\left(\mathrm{A} \beta_{42}\right)$, total 
tau ( $\mathrm{t}$-tau) and phosphorylated tau (p-tau) were measured with Elecsys immunoassays on the automated cobas e 601 analyzer. ${ }^{23}$

\section{APOE genotyping}

The Knight ADRC Genetics Core performed DNA extraction and APOE genotyping ${ }^{24}$ from non-fasted blood collected at the time of clinical assessment. APOE genotype was dichotomized as APOE $\varepsilon 4$ carrier or non-carrier.

\section{Biomarker classification}

For cross-sectional analyses assessing biomarker groups, frequency of risk factors and cognitive performance and decline, we classified participants into four groups based on combinations of baseline amyloid and tau status. Amyloid positivity $(\mathrm{A}+)$ was defined as baseline CSF $A \beta_{42}<1089 \mathrm{pg} / \mathrm{ml}$. This cut-off was determined by comparing CSF results to results of in vivo mean cortical amyloid load via positron emission tomography (PET) using ${ }^{11} \mathrm{C}$-Pittsburgh Compound B (PIB) in a separate but overlapping cohort (total $\mathrm{n}=200) .{ }^{23}$ Participants were classified as tau positive $(\mathrm{T}+)$ based on either abnormal CSF t-tau ( $>255 \mathrm{pg} / \mathrm{ml}$ ) or p-tau ( $>23 \mathrm{pg} / \mathrm{ml}$ ) at baseline, based on a cut-off (defined by Youden index) that best discriminated the reference group $(\mathrm{CDR}=0$, amyloid PET negative, $\mathrm{n}=216)$ and symptomatic $\mathrm{AD}(\mathrm{CDR}>0$, amyloid PET positive, $\mathrm{n}=52)$ in a separate but overlapping cohort (unpublished data).

For longitudinal analyses assessing within-person change in CSF biomarker values over time, we used continuous values of $\mathrm{A} \beta_{42}$, t-tau and $\mathrm{p}$-tau. For sub analyses, we stratified continuous biomarker values by baseline amyloid status.

\section{Statistical analyses}

Demographics were compared among the four biomarker groups using t-tests for continuous variables and Chi-square for categorical variables. Frequency of risk factors were compared among groups using logistic regression, adjusted for age, gender, years of education and $A P O E \& 4$ status. When the overall difference among the four groups reached significance, we conducted post-hoc testing to examine which groups differed. General linear mixed models (GLMM), with random intercepts and slopes, were used to analyze the influence of risk factor status on continuous concentrations of CSF A $\beta_{42}$, t-tau and ptau over time. For these models, the intercept was estimated based on the total sample $(n=433)$, while the slopes were estimated based on a subgroup of subjects for which longitudinal CSF markers were available $(n=184)$. For sub analyses, we also tested the inter- 
actions with baseline amyloid status. Prior to comparisons by risk factor status, $A \beta_{42}$ values were log-transformed to approximate a normal distribution, but untransformed values were used for visualization.

GLMM was also used to examine the influence of risk factors on cognitive performance and decline (outcome measures: MMSE, CDR sum of boxes and the previously mentioned cognitive composite score). For the models assessing cognition, the main effect of baseline risk factors on cognitive performance (baseline) and decline (slope) was assessed first. Thereafter, the interaction between risk factor status and baseline AD biomarker group was added to the model. In the longitudinal analyses (change in cognition and change in biomarker values), we only assessed risk factors that had an overall frequency of $>10 \%$ in the assessed group in order to have sufficient statistical power to estimate change over time. All models were adjusted for age, years of education, gender and APOE $\varepsilon 4$ positivity. The FRS was dichotomized in low $(<12.95)$ and high $(\geq 12.95)$ scores using a median split. Due to the exploratory nature of the study, we did not correct for multiple comparisons. Statistical analyses were performed using R Statistical Software (version 3.3.3) and SPSS (version 24), with significance defined as $\mathrm{p}<0.05$.

\section{RESULTS}

We included 433 individuals with an average age of 68.3 (SD 8.5) years at the time of baseline assessment. Two hundred and twenty-nine (53\%) were female and 149 (34\%) had at least one $A P O E \varepsilon 4$ allele. The average clinical follow-up time was 5.2 (SD 2.7) years, and the average biomarker follow-up time was 2.1 (SD 2.8) years. Baseline sample characteristics and frequency of risk factors by biomarker groups are shown in Table 1. One hundred and eighty-seven participants (43\%) were classified as A-T-, 72 (16\%) as A-T+, $116(27 \%)$ as $\mathrm{A}+\mathrm{T}$ - and $58(13 \%)$ as $\mathrm{A}+\mathrm{T}+\mathrm{T}+$ individuals were older compared to $\mathrm{T}$ individuals $(\mathrm{p}<0.001)$, while $\mathrm{A}+$ individuals were more likely to carry an APOE $\varepsilon 4$ allele than A- subjects $(\mathrm{p}<0.001)$. There were no differences in years of education $(\mathrm{p}=0.321)$ or in length of clinical follow-up ( $\mathrm{p}=0.071)$ among the biomarker groups (Table 1).

\section{Frequency of risk factors in baseline biomarker groups}

Overall, the most common risk factors were hypercholesterolemia (65\%), hypertension (58\%) and depression (36\%) (Table 1). Only 34 (8\%) individuals had none of the assessed risk factors, 87 (20\%) had a single risk factor and $312(72 \%)$ had more than one risk factor. The frequency of obesity was lower in the $\mathrm{T}+$ groups compared to the $\mathrm{T}$ - groups $(\mathrm{p}<0.001)$. The frequency of cardiovascular disorders was higher in the $\mathrm{A}+\mathrm{T}+$ group, relative to the biomarker-negative group $(p=0.020)$. There were no differences among the 
groups on the other individual risk factors, average number of co-occurring risk factors or the FRS (Table 1).

Table 1: Demographics and risk factors by biomarker groups

\begin{tabular}{|c|c|c|c|c|c|c|}
\hline Characteristics & $\begin{array}{l}\text { All } \\
\mathrm{n}=433\end{array}$ & $\begin{array}{l}\text { A-T- } \\
n=187\end{array}$ & $\begin{array}{l}\mathrm{A}-\mathrm{T}+ \\
\mathrm{n}=72\end{array}$ & $\begin{array}{l}A+T- \\
n=116\end{array}$ & $\begin{array}{l}\mathrm{A}+\mathrm{T}+ \\
\mathrm{n}=58\end{array}$ & $\begin{array}{l}\text { Overall } \\
\text { p-value }\end{array}$ \\
\hline Age (years) & $68.3 \pm 8.5$ & $66.1 \pm 8.7^{\mathrm{B}, \mathrm{D}}$ & $70.4 \pm 8.7^{\mathrm{A}, \mathrm{C}}$ & $68.0 \pm 8.7^{\mathrm{B}, \mathrm{D}}$ & $73.3 \pm 7.4^{\mathrm{A}, \mathrm{C}}$ & $<0.001$ \\
\hline Gender ( $\%$ female) & 53 & $57^{\mathrm{C}}$ & $67^{\mathrm{C}, \mathrm{D}}$ & $40^{\mathrm{A}, \mathrm{B}}$ & $48^{\mathrm{B}}$ & 0.001 \\
\hline APOE $\varepsilon 4$ carrier $(\%)$ & 34 & $21^{\mathrm{C}, \mathrm{D}}$ & $21^{\mathrm{C}, \mathrm{D}}$ & $49^{\mathrm{A}, \mathrm{B}}$ & $64^{\mathrm{A}, \mathrm{B}}$ & $<0.001$ \\
\hline Education (years) & $16.1 \pm 2.7$ & $16.2 \pm 2.6$ & $15.8 \pm 2.5$ & $16.3 \pm 2.9$ & $15.6 \pm 3.1$ & 0.321 \\
\hline $\begin{array}{l}\text { Biomarker follow-up (No. of } \\
\text { visits) }\end{array}$ & $1.7 \pm 0.9$ & $1.8 \pm 1.0^{\mathrm{B}, \mathrm{C}}$ & $1.5 \pm 0.9^{\mathrm{A}}$ & $1.5 \pm 0.8^{\mathrm{A}}$ & $1.6 \pm 0.8$ & 0.016 \\
\hline $\begin{array}{l}\text { Biomarker follow-up time } \\
\text { (years) }\end{array}$ & $2.1 \pm 2.8$ & $2.6 \pm 3.0$ & $1.9 \pm 2.9$ & $1.8 \pm 2.5$ & $1.7 \pm 2.5$ & 0.064 \\
\hline $\begin{array}{l}\text { Cognitive follow-up (No. of } \\
\text { visits) }\end{array}$ & $4.8 \pm 2.5$ & $4.8 \pm 2.7$ & $5.5 \pm 2.3$ & $4.4 \pm 2.3$ & $4.8 \pm 2.6$ & 0.054 \\
\hline Cognitive follow-up time (years & $5.2 \pm 2.7$ & $5.5 \pm 2.9$ & $5.5 \pm 2.2$ & $4.7 \pm 2.6$ & $4.9 \pm 2.7$ & 0.071 \\
\hline Hypertension (\%) & 58 & 49 & 64 & 64 & 67 & 0.058 \\
\hline Hypercholesterolemia (\%) & 65 & 63 & 76 & 61 & 62 & 0.206 \\
\hline Diabetes Mellitus (\%) & 9 & 8 & 10 & 12 & 2 & 0.752 \\
\hline Vitamin B12 deficiency (\%) & 3 & 1 & 3 & 5 & 2 & 0.581 \\
\hline Thyroid disease (\%) & 14 & 13 & 16 & 15 & 16 & 0.311 \\
\hline Depression (\%) & 36 & 39 & 41 & 32 & 29 & 0.613 \\
\hline Current smoking (\%) & 7 & 7 & 7 & 7 & 5 & 0.887 \\
\hline Alcohol abuse (\%) & 5 & 2 & 7 & 11 & 2 & 0.060 \\
\hline TIA (\%) & 2 & 2 & 4 & 1 & 5 & 0.871 \\
\hline Stroke (\%) & 1 & 1 & 1 & 1 & 2 & 0.809 \\
\hline Obesity (\%) & 26 & $32^{\mathrm{B}, \mathrm{D}}$ & $17^{\mathrm{A}, \mathrm{C}}$ & $32^{\mathrm{B}, \mathrm{D}}$ & $9^{\mathrm{A}, \mathrm{C}}$ & 0.048 \\
\hline Cardiovascular disorders (\%) & 13 & $10^{\mathrm{D}}$ & 15 & 11 & $26^{\mathrm{A}}$ & 0.010 \\
\hline Number of co-occurring factors & $2.4 \pm 1.4$ & $2.2 \pm 1.3$ & $2.5 \pm 1.5$ & $2.5 \pm 1.6$ & $2.2 \pm 1.3$ & 0.203 \\
\hline Framingham risk score & $16.3 \pm 11.4$ & $14.0 \pm 9.6$ & $17.2 \pm 10.6$ & $18.0 \pm 13.3$ & $18.7 \pm 12.0$ & 0.292 \\
\hline
\end{tabular}

Results are mean \pm SD for continues variables or frequency (\%). Abbreviations: $\mathrm{A}=$ amyloid, $A P O E=$ Apolipoprotein $\mathrm{E}, \mathrm{T}=\mathrm{tau}, \mathrm{TIA}=$ Transient Ischemic Attack. Comparisons between groups on frequency of risk factors were adjusted for age, gender, years of education and $A P O E \varepsilon 4+$. When overall difference between groups was significant, post-hoc testing between groups was conducted. ${ }^{\mathrm{A}} \mathrm{p}<0.05$ compared to $\mathrm{A}-\mathrm{T}-,{ }^{\mathrm{B}} \mathrm{p}<0.05$ compared to $\mathrm{A}-\mathrm{T}+,{ }^{\mathrm{C}} \mathrm{p}<0.05$ compared to $\mathrm{A}+\mathrm{T}-,{ }^{\mathrm{D}} \mathrm{p}<0.05$ compared to $\mathrm{A}+\mathrm{T}+$.

\section{Influence of risk factors on longitudinal changes in biomarker values}

Table 2 shows the effects of hypertension, hypercholesterolemia, depression, obesity, cardiovascular disorders and the FRS on $\mathrm{A} \beta_{42}$, t-tau and p-tau values at baseline and over time. Risk factors associated with significant changes in biomarker concentrations are illustrated in Figure 1. Only obesity was associated with lower t-tau $(\mathrm{p}<0.001)$ and $\mathrm{p}$-tau 
$(\mathrm{p}<0.001)$ values at baseline. There were no associations between risk factors and $A \beta_{42}$ levels at baseline. Longitudinal analyses in the entire group showed that $A \beta_{42}$ concentrations decreased significantly over time $(\beta=-50.0, p<0.001)$, while $t$-tau and $p$-tau values only increased significantly over time in individuals who were $\mathrm{A}+$ at baseline (in $\mathrm{A}+\mathrm{t}$ $\operatorname{tau} \beta=4.7, p=0.002 ; \mathrm{p}$-tau $\beta=0.6, \mathrm{p}<0.001)$. We found that none of the factors were associated with a change in $A \beta_{42}$ levels over time (Table 2). However, when stratifying by baseline amyloid status, hypertension tended to be associated with a faster rate of decrease in $A \beta_{42}$ levels only in $A$ - individuals $(\beta=-0.21, p=0.089)$ (Figure 1). Furthermore, hypertension was associated with a faster increase in $\mathrm{t}$-tau $(\beta=5.1, \mathrm{p}=0.013)$ and $\mathrm{p}$-tau $(\beta=0.5$, $\mathrm{p}=0.035$ ) levels over time. This association was influenced by baseline amyloid status, as this effect of hypertension was only present in A+ individuals (3-way interaction hypertension ${ }^{\star}$ baseline amyloid status ${ }^{\star}$ time on $\mathrm{t}$-tau $\mathrm{p}<0.001$ and $\mathrm{p}$-tau $\left.\mathrm{p}=0.006\right)$ (Figure 1 ). The absence of obesity was associated with a faster increase in $p$-tau levels over time $(\beta=0.01$, $\mathrm{p}=0.031$ ), regardless of baseline amyloid status (Figure 1). Similar to hypertension, a higher FRS was associated with a faster increase in levels of $\mathrm{t}$-tau $(\mathrm{p}=0.034)$ and $\mathrm{p}$-tau $(\mathrm{p}=0.041)$. This association was again influenced by baseline amyloid status as a faster increase rate of t-tau levels was only observed in A+ individuals (3-way interaction FRS ${ }^{\star}$ baseline amyloid status ${ }^{\star}$ time $\mathrm{p}=0.036$ ), with a similar trend for $\mathrm{p}$-tau (3-way interaction $\mathrm{FRS}^{\star}$ baseline amyloid status ${ }^{\star}$ time $\mathrm{p}=0.068$ ). Hypercholesterolemia, depression and cardiovascular disorders were not associated with longitudinal change in $A \beta_{42, t}$-tau or p-tau.

\section{Influence of risk factors on cognitive performance and decline}

We next assessed the influence of hypertension, hypercholesterolemia, thyroid disease, depression, obesity, cardiovascular disorders and the FRS on cognitive performance and decline in the entire group and as a function of baseline biomarker status. Hypertension $(p=0.041)$ and a higher FRS $(p=0.001)$ were associated with lower baseline MMSE scores in the $\mathrm{A}+\mathrm{T}+$ group, while hypercholesterolemia $(\mathrm{p}=0.039)$ and depression $(\mathrm{p}=0.021)$ were associated with higher baseline MMSE scores in the A+T+ group (Table 3, Figure 2). None of the other assessed risk factors influenced baseline MMSE scores. Longitudinal analyses showed that hypercholesterolemia was associated with a slower rate of decline ( $\beta=0.07, p=0.033$ ) of MMSE scores in the total group, while a higher FRS was associated with a faster rate of decline in the $\mathrm{A}+\mathrm{T}+$ group $(\beta=-0.24, \mathrm{p}=0.026)$. The influence of cardiovascular disorders on MMSE performance over time differed among the biomarker groups as it was associated with an increase in cognitive performance in the A+T-group, but a decrease in the other three groups (3-way interaction cardiovascular disorders ${ }^{\star} \mathrm{AT}$ groups ${ }^{\star}$ time $\left.\mathrm{p}<0.001\right)$ (Table 3, Figure 2). 


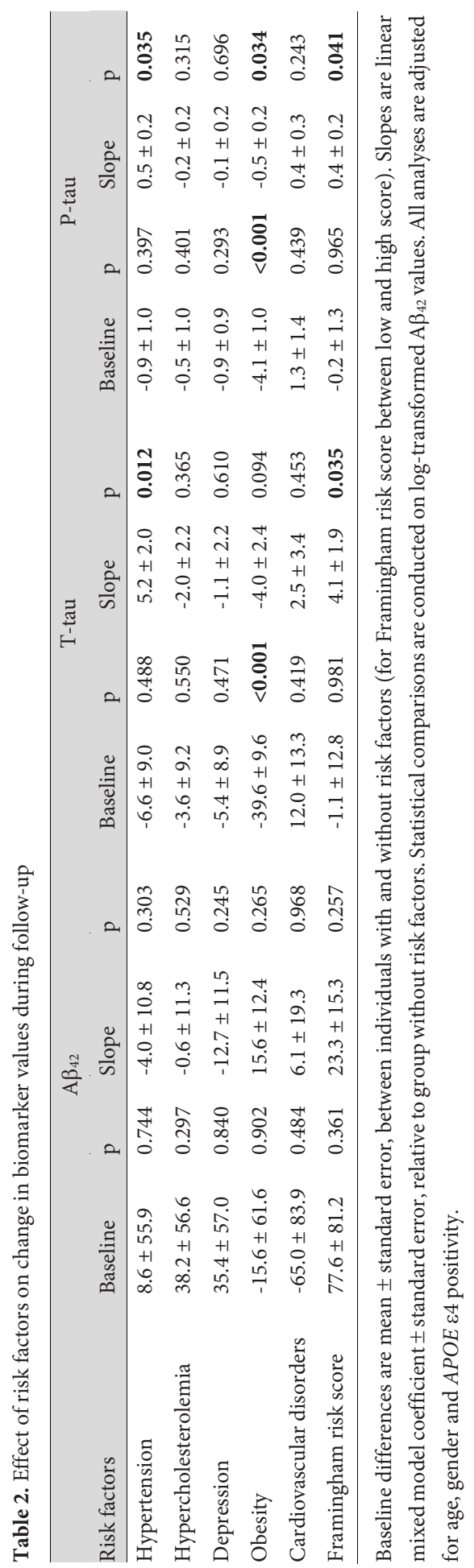


A. Hypertension
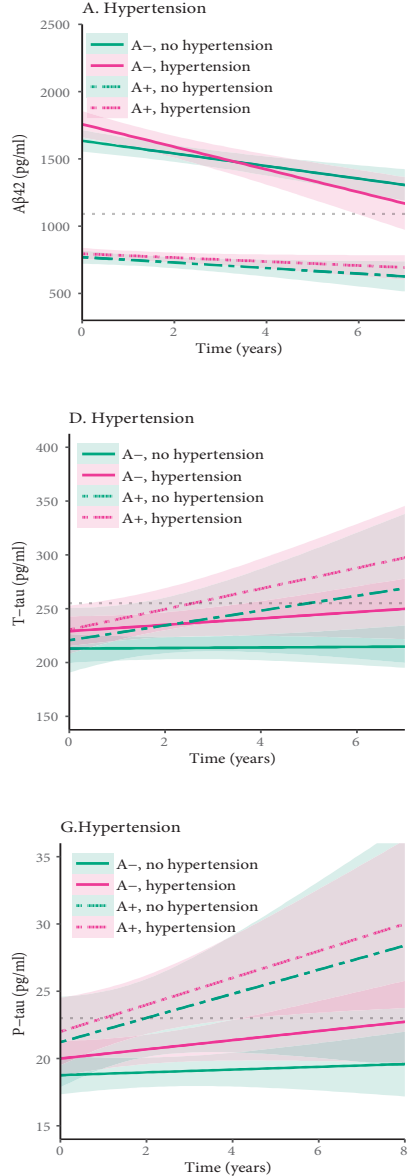

$\mathrm{A} \beta 42$
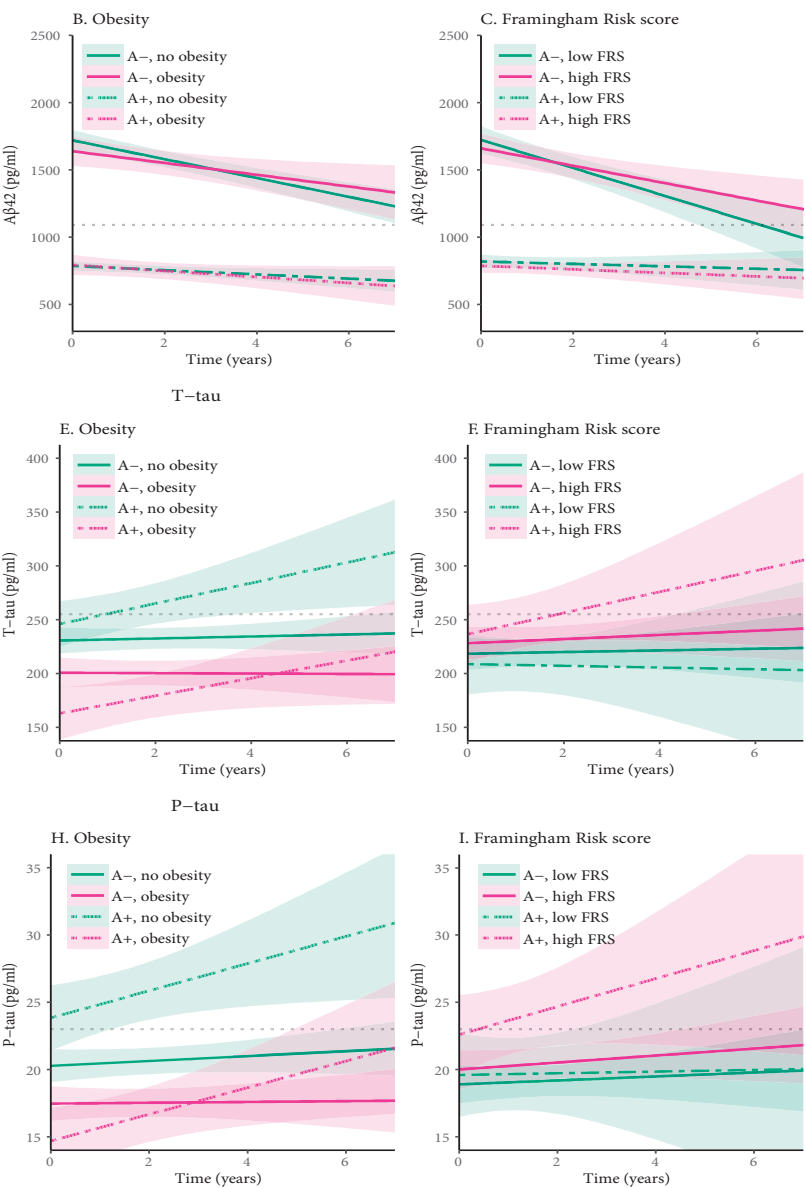

Figure 1. Longitudinal change in CSF biomarker values over time by risk factor and A $\beta$ status

The panels represent the mean scores and 95\% confidence intervals of biomarker values during follow-up by baseline amyloid status and risk factor status. The upper panels show results for $A \beta_{42}$, the middle panels for $t-$ tau, and the lower panels for p-tau. The panels on the left show the effects of hypertension, the middle panels show the effect of obesity and the panels on the right show the effect of the Framingham Risk score.

No associations with baseline cognitive performance or decline were found for the other risk factors. Results were similar when CDR sum of boxes was used as cognitive outcome measure. When using the cognitive composite score as the outcome measure, baseline results were also generally similar to the results found with MMSE except that we now also found that obesity was associated with lower baseline cognitive composite scores in the A-T-group (baseline difference: $-0.21, \mathrm{p}=0.025$ ). Longitudinally, we found no significant associations between risk factors and decline on the cognitive composite score (data not shown). When repeating all analyses after adjusting for the number of co-occurring risk factors in order to adjust for individual differences in overall vascular load, results were similar. 


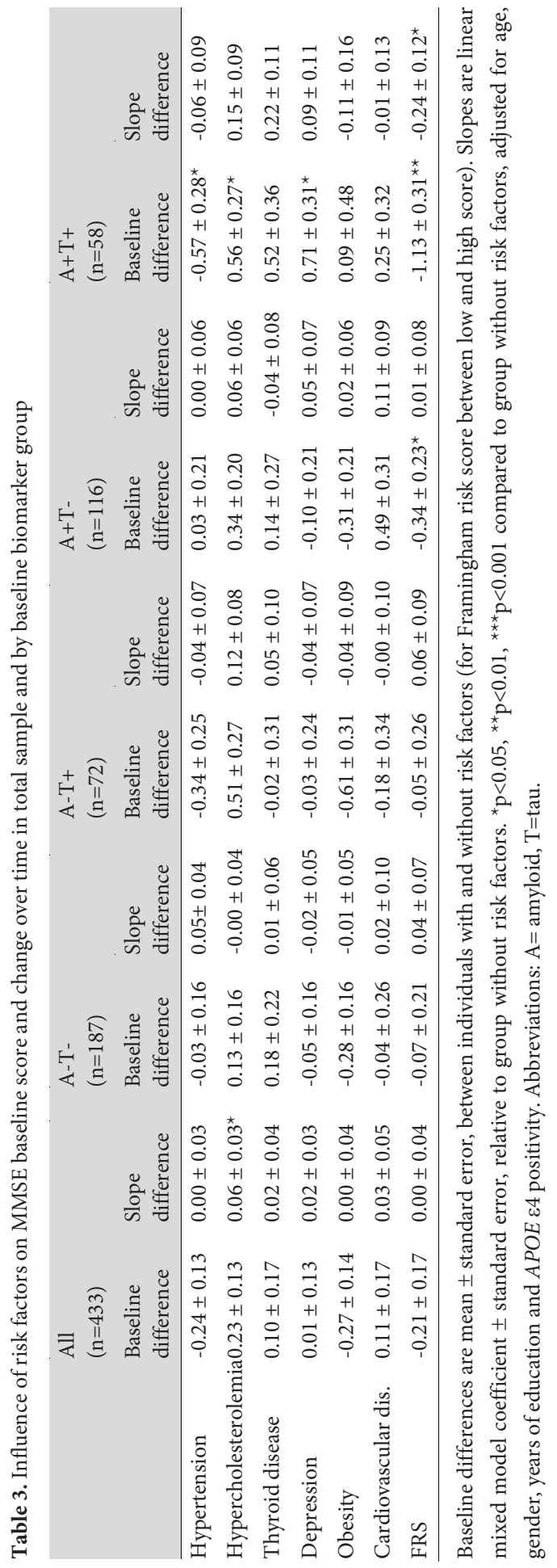




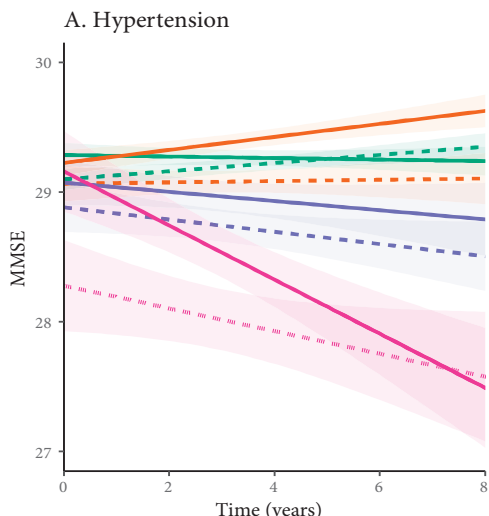

C. Depression

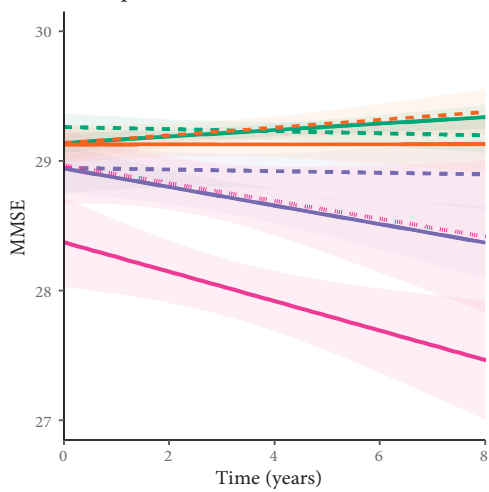

B. Hypercholesterolemia

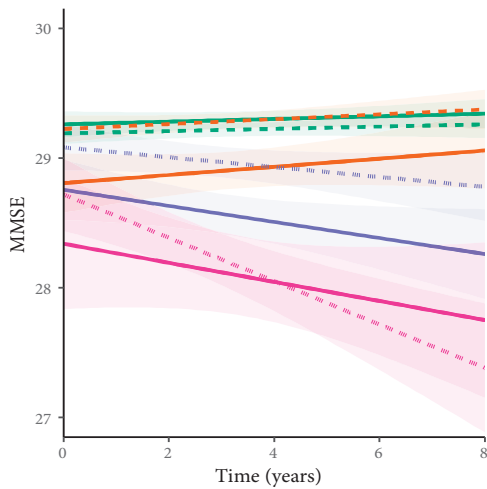

D. Framingham risk score

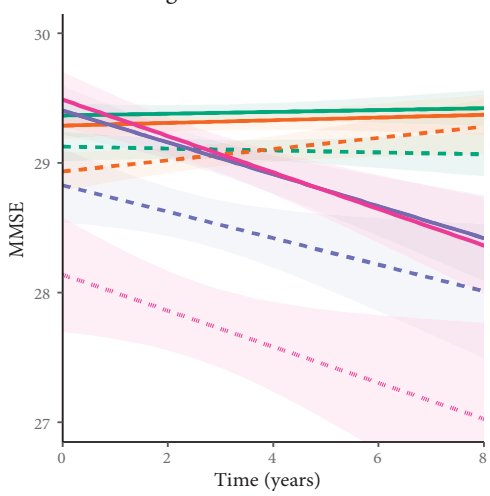

Biomarker groups

- A-T-

$-\mathrm{A}-\mathrm{T}+$

$-\mathrm{A}+\mathrm{T}-$

$-\mathrm{A}+\mathrm{T}+$

Risk factor

- Absent/Low

n't" "'? Present/High

Figure 2. Cognitive decline by risk factors status and biomarker groups

The panels represent the mean scores and 95\% confidence intervals of cognitive decline (MMSE scores) for the four biomarker groups and by presence of the risk factors. Panel A shows the effect of hypertension. Panel B shows the effect of hypercholesterolemia. Panel C shows the effect of depression. Panel D shows the effect of the Framingham risk score.

\section{DISCUSSION}

In a large cohort of cognitively normal older individuals we investigated associations of latelife risk factors with AD biomarkers profiles, longitudinal CSF biomarker changes, and cognition. Our main findings were: 1) Normal-to-low BMI was associated with abnormal t-tau and p-tau at baseline and a faster increase rate in p-tau levels over time; 2) cardiovascular disorders occurred more frequently in individuals with abnormal amyloid and tau (preclinical $A D$ ) at baseline; 2) hypertension and a higher FRS were associated with a faster increase in both p-tau and t-tau levels over time in individuals who already have amyloid pathology; and 4) hypertension and a higher FRS were inversely associated with cognitive performance, while hypercholesterolemia and depression were positively associated with cognition. 
Considering baseline biomarker profiles, we found that a lower frequency of obesity was associated with tau abnormality, suggesting that a decrease in BMI could be indicative of underlying $\mathrm{AD}$ pathology in late life. ${ }^{5,12,25-27}$ Although all individuals included in the current study were cognitively normal at baseline, the lower frequency of obesity in individuals with abnormal tau biomarkers could be due to a reduced food intake caused by subtle problems in memory. However, it is also possible that weight loss is induced by underlying metabolic or inflammatory changes associated with early AD. ${ }^{28,29}$ Furthermore, we found that cardiovascular disorders, like carotid artery stenosis and congestive heart failure, occurred more frequently in individuals with preclinical AD. This is in line with studies showing that $\mathrm{AD}$ pathology and vascular disorders, and concomitant cerebral vascular pathology, often co-exist in late-onset $\mathrm{AD} .^{30,31}$

To our knowledge, this study is among the first to investigate the influence of risk factors on longitudinal change in CSF biomarker values in cognitively normal individuals. Moreover, as a single lot of assays for each analyte $\left(A \beta_{42}\right.$, t-tau and $p$-tau) was used on a fully automated system, potential variability due to analyzing procedures is minimized in the current data set. ${ }^{23}$ The longitudinal biomarker analyses showed that both hypertension and a higher FRS were associated with a faster increase in $\mathrm{p}$-tau and t-tau concentrations over time, and this effect was driven by the individuals who already had amyloid pathology. As blood pressure is a major contributor in the FRS, ${ }^{15}$ both associations can essentially be attributed to hypertension. These findings are in line with previous animal and neuropathological studies ${ }^{32,33}$ and partly overlap with a smaller clinical study showing that a change in blood pressure was associated with an increase in p-tau concentrations over time in older individuals with hypertension. ${ }^{8}$ Normal-to-low BMI (i.e. no obesity) was associated with a faster increase in p-tau levels over time, supporting our hypothesis that weight loss might be indicative of early stage (preclinical) AD. ${ }^{28,29}$ In general we found no associations between $A \beta_{42}$ levels and vascular risk factors, suggesting separate pathophysiological amyloid and vascular pathways that both enhance neurodegeneration. ${ }^{34,35}$ However, the trend we observed regarding the influence of hypertension on change in $A \beta_{42}$ levels in amyloid-negative individuals could indicate that hypertension only influences $\mathrm{A} \beta_{42}$ levels very early in the disease process.

Hypertension and a high FRS were not only associated with changes in t-tau and p-tau values over time but also with an increased rate of cognitive decline all in individuals who already had amyloid pathology. This suggests that tau-related pathology is an important contributor to cognitive impairment, ${ }^{36,37}$ and could be a potential mediator in the relationship between amyloid, vascular factors and cognitive decline. ${ }^{35}$ Hypercholesterolemia and depression were both associated with higher cognitive scores at baseline in the A+T+ group, and hypercholesterolemia was also associated with a slower decrease in cognitive 
performance over time. Although these findings seem counterintuitive, they are consistent with results from our previous study in individuals with mild cognitive impairment (MCI), ${ }^{12}$ as well as with other studies suggesting that a decline in total cholesterol levels may be associated with early stage dementia. ${ }^{38,39}$ While the pathophysiological relation between $\mathrm{AD}$ and cholesterol remains uncertain, synaptic failure could be involved as this has been related an imbalance of cholesterol homeostasis, as well as to early AD and cognitive decline. ${ }^{40-42}$ Regarding depression, severity and trajectory of the depressive symptoms could temporarily impact cognitive performance in preclinical AD. ${ }^{43}$ The fact that most of our significant associations regarding cognition concern only the $\mathrm{A}+\mathrm{T}+$ group, is consistent with the hypothesized temporal ordering of pathological and clinical events regarding AD. ${ }^{44,45}$ In addition, we found that the MMSE and CDR were more sensitive in detecting cognitive decline compared to a cognitive composite score consisting of five cognitive measures, including a memory task. This observation may reflect the fact that the MMSE and CDR, in contrast to the cognitive composite score, also measure functional status including orientation in time and place, measures which have been found to be sensitive in detecting preclinical $\mathrm{AD}$ and cognitive decline. ${ }^{46,47}$

Our study has several limitations that should be mentioned. First, data on risk factors and medication use were based on self or proxy-reported information which could have led to under or over reporting of risk factors. Second, we were unable to assess the influence of all risk factors on change in CSF biomarkers and cognition over time as the overall frequency was too low for several factors. This low overall frequency of risk factors may be due to baseline exclusion of individuals with a medical or psychiatric illness (e.g. renal failure requiring dialysis, use of insulin, or depression requiring electroconvulsive therapy) that could interfere with longitudinal follow-up or adversely impact cognition. Third, we only investigated relationships with CSF biomarkers, and imaging markers (i.e. amyloid PET or tau PET) could have led to different results. Lastly, as this study included individuals who were willing to participate in biomarker studies, the frequencies of vascular risk and of AD biomarkers found in this sample are not directly comparable to those in the general population. The major strengths of our study include the relatively long clinical follow-up, the diverse spectrum of assessed risk factors and the unique data on longitudinal CSF measurements, analyzed using an automated system, in a large cohort of healthy volunteers.

In conclusion, we found that hypertension and a higher vascular load enhanced neurodegeneration in cognitively normal individuals who already had amyloid pathology. In addition, a normal-to-low BMI may be related to early AD given its association with increased tau levels. These data support the view that it is important to initiate treatment and management of hypertension early in life, while other factors, such as BMI, should be monitored from midlife onwards to detect early changes possibly related to AD. 


\section{REFERENCES}

1. de Bruijn RF, Ikram MA. Cardiovascular risk factors and future risk of Alzheimer's disease. BMC Med. 2014;12:130.

2. Gelber RP, Launer LJ, White LR. The Honolulu-Asia Aging Study: epidemiologic and neuropathologic research on cognitive impairment. Curr Alzheimer Res. 2012;9(6):664-672.

3. Mielke MM, Rosenberg PB, Tschanz J, et al. Vascular factors predict rate of progression in Alzheimer disease. Neurology. 2007;69(19):1850-1858.

4. Whitmer RA, Sidney S, Selby J, Johnston SC, Yaffe K. Midlife cardiovascular risk factors and risk of dementia in late life. Neurology. 2005;64(2):277-281.

5. Gottesman RF, Schneider AL, Zhou Y, et al. Association Between Midlife Vascular Risk Factors and Estimated Brain Amyloid Deposition. Jama. 2017;317(14):1443-1450.

6. Vemuri P, Knopman DS, Lesnick TG, et al. Evaluation of Amyloid Protective Factors and Alzheimer Disease Neurodegeneration Protective Factors in Elderly Individuals. JAMA Neurol. 2017.

7. Vemuri P, Lesnick TG, Przybelski SA, et al. Age, Vascular Health, and Alzheimer's disease Biomarkers in an Elderly Sample. Annals of neurology. 2017.

8. Glodzik L, Rusinek H, Pirraglia E, et al. Blood pressure decrease correlates with tau pathology and memory decline in hypertensive elderly. Neurobiology of aging. 2014;35(1):64-71.

9. Lo RY, Jagust WJ, Alzheimer's Disease Neuroimaging I. Vascular burden and Alzheimer disease pathologic progression. Neurology. 2012;79(13):1349-1355.

10. Enache D, Solomon A, Cavallin L, et al. CAIDE Dementia Risk Score and biomarkers of neurodegeneration in memory clinic patients without dementia. Neurobiology of aging. 2016;42:124-131.

11. Kemppainen N, Johansson J, Teuho J, et al. Brain amyloid load and its associations with cognition and vascular risk factors in FINGER Study. Neurology. 2018;90(3):e206-e213.

12. Bos I, Vos SJ, Frölich L, et al. The frequency and influence of dementia risk factors in prodromal Alzheimer's disease. Neurobiology of aging. 2017.

13. Coats M, Morris JC. Antecedent biomarkers of Alzheimer's disease: the adult children study. Journal of geriatric psychiatry and neurology. 2005;18(4):242-244.

14. Morris JC. The Clinical Dementia Rating (CDR): current version and scoring rules. Neurology. 1993;43(11):2412-2414

15. D'Agostino RB, Sr., Vasan RS, Pencina MJ, et al. General cardiovascular risk profile for use in primary care: the Framingham Heart Study. Circulation. 2008;117(6):743-753.

16. Grober E, Buschke H, Crystal H, Bang S, Dresner R. Screening for dementia by memory testing. Neurology. 1988;38(6):900-903.

17. Reitan R. Trail-Making Test. Arizona: Reitan Neuropsychology Laboratory 1979.

18. Goodglass H, Kaplan, E. The assessment of aphasia and related disorders. . Philadelphia: Lea \& Febiger; 1972.

19. Wechsler D. Manual: Wechsler Adult Intelligence Scale - Revised. New York: Psychological Corporation; 1981.

20. Johnson DK, Storandt M, Morris JC, Galvin JE. Longitudinal study of the transition from healthy aging to Alzheimer disease. Archives of neurology. 2009;66(10):1254-1259.

21. Folstein MF, Folstein SE, McHugh PR. "Mini-mental state". A practical method for grading the cognitive state of patients for the clinician. Journal of psychiatric research. 1975;12(3):189-198.

22. Fagan AM, Mintun MA, Mach RH, et al. Inverse relation between in vivo amyloid imaging load and cerebrospinal fluid Abeta42 in humans. Annals of neurology. 2006;59(3):512-519.

23. Schindler SE, Gray JD, Gordon BA, et al. Cerebrospinal fluid biomarkers measured by Elecsys assays compared to amyloid imaging. Alzheimer's \& dementia : the journal of the Alzheimer's Association. 2018. 
24. Pastor P, Roe CM, Villegas A, et al. Apolipoprotein Eepsilon4 modifies Alzheimer's disease onset in an E280A PS1 kindred. Annals of neurology. 2003;54(2):163-169.

25. Muller S, Preische O, Sohrabi HR, et al. Decreased body mass index in the preclinical stage of autosomal dominant Alzheimer's disease. Scientific reports. 2017;7(1):1225.

26. Besser LM, Alosco ML, Ramirez Gomez L, et al. Late-Life Vascular Risk Factors and Alzheimer Disease Neuropathology in Individuals with Normal Cognition. Journal of neuropathology and experimental neurology. 2016;75(10):955-962.

27. Vidoni ED, Townley RA, Honea RA, Burns JM. Alzheimer disease biomarkers are associated with body mass index. Neurology. 2011;77(21):1913-1920.

28. Cai H, Cong WN, Ji S, Rothman S, Maudsley S, Martin B. Metabolic dysfunction in Alzheimer's disease and related neurodegenerative disorders. Curr Alzheimer Res. 2012;9(1):5-17.

29. Mahieux F, Couderc R, Fenelon G, Maachi M. [Relationships between weight loss and circulating cytokines in patients with Alzheimer's disease]. Psychologie \& neuropsychiatrie du vieillissement. 2006;4(4):281-286.

30. Attems J, Jellinger KA. The overlap between vascular disease and Alzheimer's disease--lessons from pathology. BMC Med. 2014;12:206.

31. Schneider JA, Aggarwal NT, Barnes L, Boyle P, Bennett DA. The neuropathology of older persons with and without dementia from community versus clinic cohorts. Journal of Alzheimer's disease : JAD. 2009;18(3):691-701.

32. Petrovitch H, White LR, Izmirilian G, et al. Midlife blood pressure and neuritic plaques, neurofibrillary tangles, and brain weight at death: the HAAS. Honolulu-Asia aging Study. Neurobiology of aging. 2000;21(1):57-62.

33. Kruyer A, Soplop N, Strickland S, Norris EH. Chronic Hypertension Leads to Neurodegeneration in the TgSwDI Mouse Model of Alzheimer's Disease. Hypertension. 2015;66(1):175-182.

34. Vemuri P, Knopman DS. The role of cerebrovascular disease when there is concomitant Alzheimer disease. Biochimica et biophysica acta. 2016;1862(5):952-956.

35. Bos I, Verhey FR, Ramakers I, et al. Cerebrovascular and amyloid pathology in predementia stages: the relationship with neurodegeneration and cognitive decline. Alzheimer's research \& therapy. 2017;9(1):101.

36. Nelson PT, Alafuzoff I, Bigio EH, et al. Correlation of Alzheimer disease neuropathologic changes with cognitive status: a review of the literature. Journal of neuropathology and experimental neurology. 2012;71(5):362-381.

37. Bejanin A, Schonhaut DR, La Joie R, et al. Tau pathology and neurodegeneration contribute to cognitive impairment in Alzheimer's disease. Brain : a journal of neurology. 2017.

38. Stewart R, White LR, Xue QL, Launer LJ. Twenty-six-year change in total cholesterol levels and incident dementia: the Honolulu-Asia Aging Study. Archives of neurology. 2007;64(1):103-107.

39. Mielke MM, Zandi PP, Sjogren M, et al. High total cholesterol levels in late life associated with a reduced risk of dementia. Neurology. 2005;64(10):1689-1695.

40. Petrov AM, Kasimov MR, Zefirov AL. Cholesterol in the Pathogenesis of Alzheimer's, Parkinson's Diseases and Autism: Link to Synaptic Dysfunction. Acta Naturae. 2017;9(1):26-37.

41. Koudinov AR, Koudinova NV. Cholesterol, synaptic function and Alzheimer's disease. Pharmacopsychiatry. 2003;36 Suppl 2:S107-112.

42. Selkoe DJ. Alzheimer's disease is a synaptic failure. Science. 2002;298(5594):789-791.

43. Mirza SS, Wolters FJ, Swanson SA, et al. 10-year trajectories of depressive symptoms and risk of dementia: a population-based study. Lancet Psychiatry. 2016;3(7):628-635. 
44. Vos SJ, Xiong C, Visser PJ, et al. Preclinical Alzheimer's disease and its outcome: a longitudinal cohort study. The Lancet Neurology. 2013;12(10):957-965.

45. Jack CR, Knopman DS, Jagust WJ, et al. Tracking pathophysiological processes in Alzheimer's disease: an updated hypothetical model of dynamic biomarkers. The Lancet Neurology. 2013;12(2):207-216.

46. Allison SL, Fagan AM, Morris JC, Head D. Spatial Navigation in Preclinical Alzheimer's Disease. Journal of Alzheimer's disease : JAD. 2016;52(1):77-90.

47. Guerrero-Berroa E, Luo X, Schmeidler J, et al. The MMSE orientation for time domain is a strong predictor of subsequent cognitive decline in the elderly. International journal of geriatric psychiatry. 2009;24(12):1429-1437. 
Chapter 5

\section{SUPPLEMENTAL DATA}

Supplemental Table 1. Definition of risk factors

\begin{tabular}{ll}
\hline Risk factors & Definition \\
\hline Hypertension & Medical history, or use of antihypertensive treatment, or a systolic blood \\
& pressure $\geq 140 \mathrm{mmHg}$, or a diastolic blood pressure $\geq 90 \mathrm{~mm} H$ \\
Hypercholesterolemia & Medical history, or use of lipid- lowering treatment, or total cholesterol $\geq 200$ \\
& $\mathrm{mg} / \mathrm{dL}$ \\
Diabetes Mellitus & Medical history, or use of diabetes treatment \\
Vitamin B12 deficiency & Medical history \\
Thyroid disease & Medical history \\
Depression & Medical history, or use of anti-depression treatment, or a score $\geq 6$ on the \\
Current smoking & GDS-15 \\
Alcohol abuse & Smoked in the last 30 days \\
TIA & Medical history \\
Stroke & Medical history \\
Obesity & Medical history \\
Cardiovascular disorders & BMI $>30$ \\
Medical history of heart attack/cardiac arrest, atrial fibrillation, & angioplasty/endarterectomy/stent, cardiac bypass procedure, pacemaker or \\
congestive heart failure & Calculated based on serum total and HDL cholesterol, systolic blood \\
pressure, smoking status and medical history of diabetes.
\end{tabular}

Abbreviations: BMI = Body Mass Index, GDS = Geriatric Depression Scale, HDL = high-density lipoprotein, TIA $=$ Transient Ischemic Attack. ${ }^{1}$ D'Agostino et al. 2008. 



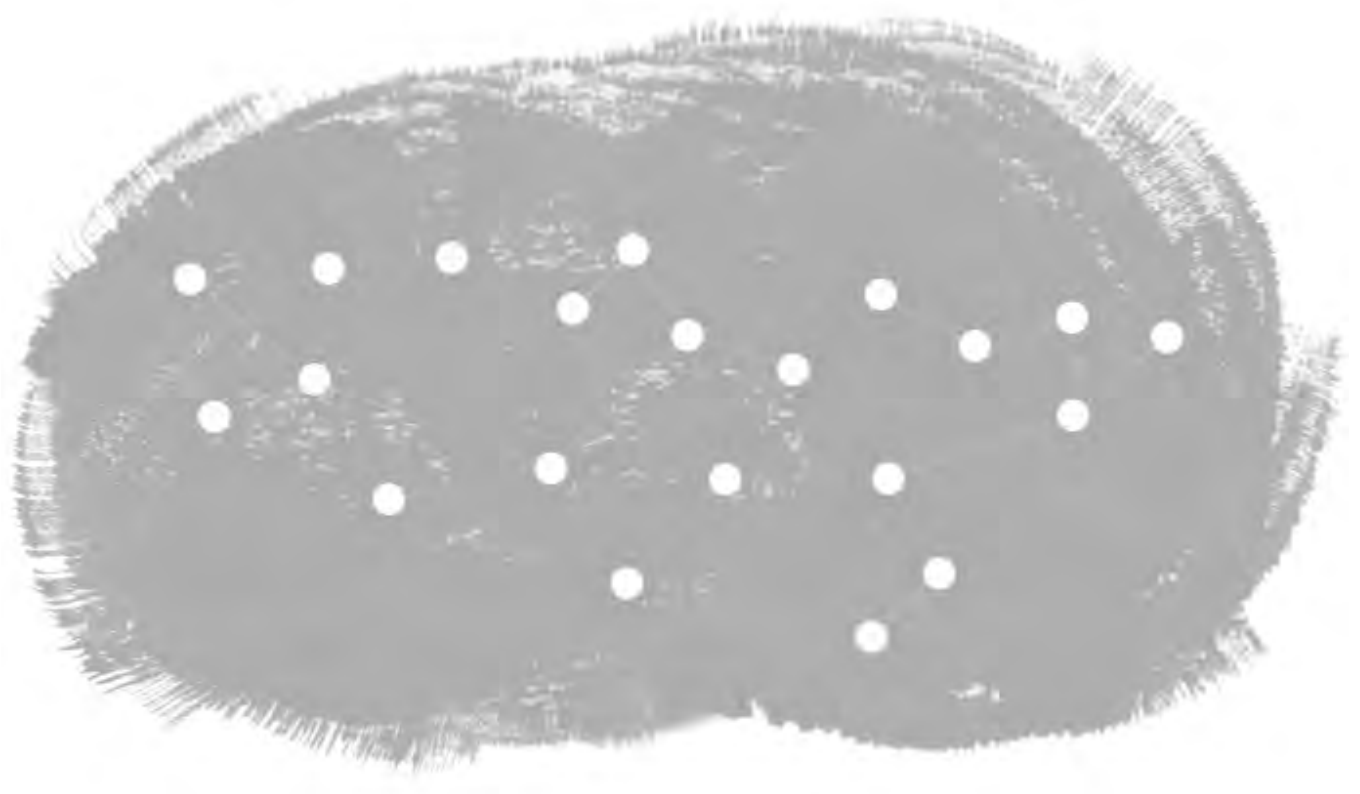




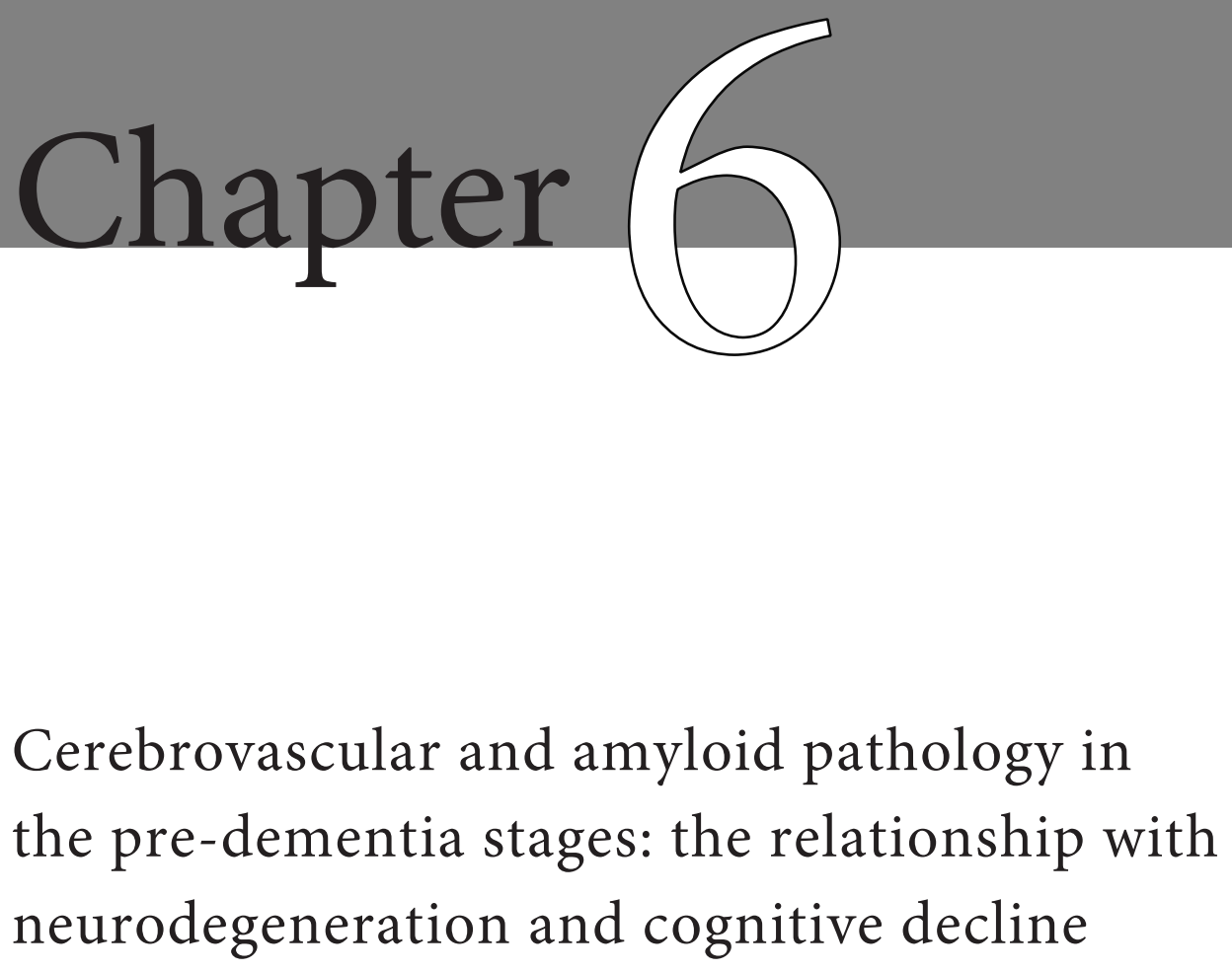

Alzheimer's Research and Therapy 2017; 9(1),101

Isabelle Bos, Frans Verhey, Inez Ramakers, Heidi I.L. Jacobs, Hilkka Soininen, Yvonne Freund-Levi, Harald Hampel, Magda Tsolaki, Åsa K. Wallin, Mark A. van Buchem, Ania Oleksik, Marcel M. Verbeek, Marcel Olde Rikkert, Wiesje M. van der Flier, Philip Scheltens, Pauline Aalten, Pieter Jelle Visser and Stephanie J.B. Vos 


\section{ABSTRACT}

Background: Cerebrovascular disease (CVD) and amyloid-beta (A $\beta)$ often co-exist, but their influence on neurodegeneration and cognition in pre-dementia stages remains unclear. We investigated the association between CVD and $A \beta$ on neurodegenerative markers and cognition in non-demented patients.

Methods: We included 271 memory-clinic patients with subjective or objective cognitive deficits but without dementia, from the BioBank Alzheimer Center Limburg cohort $(n=99)$ and the LeARN $(n=50)$ and DESCRIPA $(n=122)$ multicenter studies. CSF A $\beta 1$ 42 and white matter hyperintensities (WMH) on MRI, were used as measures of $A \beta$ and $\mathrm{CVD}$, respectively. Individuals were classified into four groups based on the presence or absence (+/-) of $\mathrm{A} \beta$ and WMH. We investigated differences in phosphorylated tau ( $\mathrm{p}$ tau), total tau ( $\mathrm{t}$-tau) and medial temporal lobe atrophy (MTA) between groups using general linear models. We examined cognitive decline and progression to dementia using linear mixed models and cox proportional hazard models. All analyses were adjusted for study and demographics.

Results: MTA and t-tau were elevated in the $\mathrm{A} \beta-\mathrm{WMH}+, \mathrm{A} \beta+\mathrm{WMH}-$ and $\mathrm{A} \beta+\mathrm{WMH}+$ groups. MTA was most severe in the $\mathrm{A} \beta+\mathrm{WMH}+$ group, compared to the groups with a single pathology. Both $\mathrm{WMH}$ and $\mathrm{A} \beta$ were associated with cognitive decline, but having both pathologies simultaneously was not associated with faster decline.

Conclusions: In the current study, we found an additive association of A $\beta$ and CVD pathology with baseline MTA, but not with cognitive decline. As our findings may have implications for diagnosis and prognosis of memory clinic patients and for future scientific research, they should be validated in a larger sample with longer follow-up. 


\section{INTRODUCTION}

Cerebrovascular disease (CVD) often co-exists with Alzheimer's disease (AD) and both conditions add to cognitive decline. ${ }^{1,2}$ The influence of co-existing CVD and AD pathology on neurodegeneration and cognitive decline in pre-dementia stages of $\mathrm{AD}$, however, remains uncertain. Understanding the role CVD pathology in early AD is key for understanding and preventing cognitive decline in $\mathrm{AD}$.

In demented subjects, co-existent $\mathrm{AD}$ and CVD pathology at autopsy is associated with more rapid cognitive decline, and often a more severe form of dementia than isolated $A D$ pathology. ${ }^{3,4} \mathrm{~A}$ combination of $\mathrm{AD}$ and $\mathrm{CVD}$ has also been associated with a lower burden of amyloid-beta $(\mathrm{A} \beta)$ pathology compared to isolated $\mathrm{AD},{ }^{5,6}$ suggesting that less $\mathrm{AD}$ pathology is needed for cognitive impairment in individuals who also have CVD. ${ }^{7,8}$ In cognitively normal subjects, it was shown that $\mathrm{A} \beta$ and CVD pathology are independent contributors to cognitive decline and both increase the risk of dementia. ${ }^{79}$ Studies on the contribution of $A \beta$ and CVD pathology on cognitive decline in individuals with subjective cognitive decline (SCD) and mild cognitive impairment (MCI) have shown conflicting results. ${ }^{10,11}$ Also, it is less well understood how each of these pathologies relate to different markers of neurodegeneration, since previous studies point in different directions or only focused on a single marker instead of investigating multiple neurodegenerative markers from various modalities (e.g. magnetic resonance imaging (MRI) and cerebrospinal fluid (CSF)). ${ }^{12-15}$ Clarity on the relationship between co-existing AD and CVD pathology, neurodegenerative markers and cognition, will improve diagnostic and prognostic accuracy of early AD.

The aim of this study is to investigate in patients with SCD and MCI whether there is an additive effect of CVD and $A \beta$ on neurodegeneration measured by total tau ( $t$-tau) and phosphorylated tau (p-tau) in CSF, and medial temporal lobe atrophy (MTA) on MRI, and on cognitive decline during follow-up.

\section{METHODS}

\section{Subjects}

Two hundred seventy-one subjects were selected from memory clinics of the single center BioBank Alzheimer Center Limburg (BBACL, $n=99)$ cohort and LeARN $(n=50)^{16}$ and DESCRIPA ( $n=122)^{17}$ multicenter studies. Inclusion criteria were: 1$)$ no diagnosis of dementia at baseline; and 2) baseline data available on MRI and CSF measures. When subjects participated in more than one study, we included the data from the study with the 
longest follow-up. The medical ethics committee at each site approved the study. All subjects provided informed consent.

\section{Clinical assessment}

Clinical assessment included neuropsychological assessment and an assessment of medical history. Information on medical history (e.g. hypertension, diabetes and obesity) was provided by patient and/or caregiver, or extracted from medical files. Neuropsychological assessment was performed according to local routine protocol of each site, including the Mini-Mental State Examination (MMSE) and at least one test in the cognitive domains of memory and executive functioning. The delayed recall of a wordlist test was used to examine memory. For the BBACL and LeARN studies the Auditory Verbal Learning Test (RAVLT) was used. ${ }^{18}$ For the DESCRIPA cohort, the RAVLT and CERAD wordlist were primary memory tests. DESCRIPA tests per center are described elsewhere. ${ }^{17}$ The Trail Making Test ${ }^{19}$ part $\mathrm{B}$ was used to examine executive functioning. Raw scores on each test were converted to $\mathrm{z}$-scores, using local normative data. Z-scores below $-5(n=7)$, were rounded to -5 to avoid a bias through outliers in the data.

Diagnosis of MCI at baseline was made according to the criteria of Petersen et al. ${ }^{20}$ Subjects with a $\mathrm{z}$-score below -1.5 on the immediate recall or delayed recall of a word list test were classified as having amnestic MCI. Subjects with a z-score below -1.5 on any of the non-memory tests, were classified as non-amnestic MCI. Diagnosis of AD-type dementia at follow-up was made according to Diagnostic and Statistical Manual of Mental Disorders, Fourth Edition ${ }^{21}$ and National Institute of Neurological and Communicative Disorders and Stroke - Alzheimer's Disease and Related Disorders Association criteria (NINCDS-ADRDA) criteria. ${ }^{22}$ Etiological diagnoses for other types of dementia were made according to standardized clinical criteria for vascular dementia, ${ }^{23}$ frontotemporal dementia, ${ }^{24}$ and Lewy body dementia. ${ }^{25}$

\section{CSF analyses}

CSF was collected by a lumbar puncture and thereafter centrifuged and stored at $-80^{\circ} \mathrm{C}$ in polypropylene tubes. CSF amyloid-beta $(A \beta) 1-42$, t-tau and p-tau were analyzed using Innotest sandwich ELISA (Innogenetics, Ghent, Belgium) in Gothenburg for the DESCRIPA cohort, ${ }^{17}$ in Amsterdam for the LeARN project ${ }^{26}$ and in Nijmegen for the BBACL study. ${ }^{27}$ To define abnormality of the CSF measures the following predefined cut-offs were used: $\mathrm{A} \beta 1-42 \leq 550 \mathrm{pg} / \mathrm{ml}, \mathrm{t}$-tau $>375 \mathrm{pg} / \mathrm{ml}$, and $\mathrm{p}-\mathrm{tau}_{181}>52 \mathrm{pg} / \mathrm{ml}^{28}$ 


\section{Genetic analyses}

The apolipoprotein E (APOE) genotype was determined in a subgroup of the sample $(n=165)$. Assessments were performed according to routine protocol at each site, as described elsewhere. ${ }^{17,29}$

\section{MRI analyses}

All subjects were scanned according to the routine MRI protocol at each site (Supplemental Text 1). Scanning was performed at a strength of $1.0(n=14), 1.5(n=108)$ or 3.0 $(\mathrm{n}=149)$ Tesla, and all scans included a 3-dimensional T1-weighted gradient echo sequence and a fast fluid-attenuated inversion recovery sequence. To determine medial temporal lobe atrophy (MTA), the visual rating Scheltens MTA scale ${ }^{30}$ was used. The score on the MTA scale ranges from 0-4 for each hemisphere. The summed score of both hemispheres was used, where an abnormal MTA was defined using a cut-off $\geq 2 .{ }^{31} \mathrm{WMH}$ were measured with the visually rated ARWMC scale (range $0-3)^{32}$ for the DESCRIPA cohort, and with the visual rated Fazekas scale (range $0-3)^{33}$ for the BBACL and LeARN cohorts. For the ARWMC, a cut-off score of $\geq 2$ in at least one of the measured brain areas was used to define WMH status. ${ }^{34,35}$ For the Fazekas scale, a cut-off score $\geq 2$ was also used to define WMH status. ${ }^{36}$

\section{Subject classification}

To classify individuals in subgroups we used $\mathrm{A} \beta$ as a measure of $\mathrm{AD}$ and $\mathrm{WMH}$ as a measure of CVD. Subjects were classified as amyloid positive (A $\beta+)$ when CSF A $\beta 1-42$ levels were abnormal. Subjects were classified as $\mathrm{WMH}$ positive $(\mathrm{WMH}+)$ when the $\mathrm{WMH}$ score was high. We created four groups based on combinations of $A \beta$ status and $W M H$ status: $\mathrm{A} \beta-\mathrm{WMH}-, \mathrm{A} \beta-\mathrm{WMH}+, \mathrm{A} \beta+\mathrm{WMH}-, \mathrm{A} \beta+\mathrm{WMH}+$.

\section{Statistical analyses}

We analyzed differences on clinical baseline and follow-up characteristics and neurodegeneration markers between groups using ANOVA for continuous variables, and chisquared tests for categorical variables. Prior to the continuous comparisons of biomarker values between groups (Table 1 and 2), A $\beta 1-42$, p-tau and t-tau values were log-transformed to approximate a normalize distribution required for statistical comparisons. The raw biomarker values are shown in the tables. Comparisons between $\mathrm{A} \beta / \mathrm{WMH}$ groups on neurodegenerative markers (Table 2) were all corrected for demographics, study and baseline diagnosis. The associations between $\mathrm{A} \beta / \mathrm{WMH}$ groups and change on the MMSE, memory and executive functioning were assessed by slope analyses with linear mixed models. The analyses included the baseline scores and all available follow-up scores 
(up to four years). All slope analyses were adjusted for study. When the interaction between $\mathrm{A} \beta / \mathrm{WMH}$ group, baseline diagnosis and time was significant, we added baseline diagnosis as a covariate to the model. The models adjusted for baseline diagnoses are reported in the tables and figures, the results stratified by diagnoses were reported in the text. For the MMSE we also adjusted for age, gender and years of education, as these scores are not standardized. We also tested the influence of APOE genotype in a subgroup of the sample for whom this was available. Models were fitted with random study specific intercept and subject specific slopes and a first-order autoregressive (AR-1) correlation structure. We choose this model because it provided the best -2 Log Likelihood ratio and the lowest number of parameters. Cox proportional hazards models were used to investigate the risk of progression to dementia for each group after adjusting for demographics, study and baseline diagnosis. Statistical analyses were conducted with SPSS version 24.0 (Chicago, IL, USA) with significance level set at $\mathrm{p}<0.05$. Due to the exploratory nature of the

study, we did not control for multiple comparisons. Post-hoc power calculations were conducted using SPSS and the 'simr' package of R statistical software (version 3.3.3).

\section{RESULTS}

\section{Cohort characteristics}

We included 271 individuals with a mean age of 65.6 (SD 9.0). One hundred sixty-five (61\%) were female and 153 (57\%) had a diagnosis of MCI at baseline, of which 99 (65\%) were classified as having amnestic MCI. For 233 individuals (86\%) follow-up data was available. The availability of follow-up data was not different amongst the $A \beta / \mathrm{WMH}$ groups ( $\mathrm{p}=0.396)$ or studies ( $\mathrm{p}=0.730$ ). After a mean follow-up time of 2.5 (SD 1.2) years, $46(17 \%)$ subjects progressed to dementia. The majority $(80 \%)$ of the individuals who progressed to dementia had a clinical diagnosis of AD-type dementia.

Table 1 shows baseline and follow-up characteristics of the four A $\beta / \mathrm{WMH}$ groups. The group without pathology was younger $(\mathrm{p}<0.001)$ and progressed less frequently to dementia compared to the other three groups $(\mathrm{p}<0.001)$. We found no difference in prevalence of several vascular risk factors between the four groups (hypertension: $\mathrm{p}=0.563$; obesity: $\mathrm{p}=0.486$; diabetes: $\mathrm{p}=0.106$ ). We found no difference in $\mathrm{A} \beta$ load between the group with only $\mathrm{A} \beta$ and the group with both $\mathrm{A} \beta$ and $\mathrm{WMH}(\mathrm{p}=0.502)$. Likewise, the proportion of $\mathrm{WMH}$, was not different between the two $\mathrm{WMH}$ positive groups $(\mathrm{A} \beta-\mathrm{WMH}+$ and $\mathrm{A} \beta+\mathrm{WMH}+, \mathrm{p}=0.175)$. 
Table 1: Comparisons of baseline and follow-up characteristics by $\mathrm{A} \beta$ and WMH status

\begin{tabular}{|c|c|c|c|c|}
\hline & $\mathrm{A} \beta-\mathrm{WMH}-$ & $\mathrm{A} \beta-\mathrm{WMH}+$ & $\mathrm{A} \beta+\mathrm{WMH}-$ & $\mathrm{A} \beta+\mathrm{WMH}+$ \\
\hline Baseline characteristics & $\mathrm{n}=140$ & $\mathrm{n}=39$ & $\mathrm{n}=63$ & $\mathrm{n}=29$ \\
\hline Age & $61.7(8.3)^{B, C, D}$ & $71.3(7.7)^{\mathrm{A}, \mathrm{C}}$ & $66.7(7.8)^{\mathrm{A}, \mathrm{B}, \mathrm{D}}$ & $74.1(5.0)^{\mathrm{A}, \mathrm{C}}$ \\
\hline Female, $\mathrm{n}$ & $94(67)^{\mathrm{C}}$ & $23(59)$ & $32(51)^{A}$ & $16(55)$ \\
\hline Education in years & $10.9(3.1)$ & $11.9(3.3)$ & $11.1(3.1)$ & $10.3(2.9)$ \\
\hline Hypertension, $\mathrm{n}^{*}$ & $43(34)$ & $9(25)$ & $15(25)$ & $9(32)$ \\
\hline Obesity, $\mathrm{n}^{*}$ & $15(14)$ & $3(11)$ & $4(8)$ & $4(21)$ \\
\hline Diabetes, $\mathrm{n}^{*}$ & $16(21)$ & $3(15)$ & $3(7)$ & $5(28)$ \\
\hline APOE- $\varepsilon 4$ carrier, $\mathrm{n}^{*}$ & $33(51)^{\mathrm{B}}$ & $5(24)^{\mathrm{A}, \mathrm{C}, \mathrm{D}}$ & $29(62)^{\mathrm{B}}$ & $10(56)^{\mathrm{B}}$ \\
\hline Diagnosis MCI, $\mathrm{n}$ & $70(50)^{\mathrm{D}}$ & $21(54)^{\mathrm{D}}$ & $40(64)$ & $22(76)^{A, B}$ \\
\hline - Amnestic MCI (\% within MCI) & $40(57)$ & $15(71)$ & $27(68)$ & $17(77)$ \\
\hline - Non-amnestic MCI (\% within MCI & $30(43)$ & $6(29)$ & $13(33)$ & $5(23)$ \\
\hline $\mathrm{CSF} A \beta 1-42, \mathrm{pg} / \mathrm{ml}$ & $973.6(312.0)^{\mathrm{C}, \mathrm{D}}$ & $885.0(242.0)^{\mathrm{C}, \mathrm{D}}$ & $404.3(102.6)^{\mathrm{A}, \mathrm{B}}$ & $419.3(97.2)^{\mathrm{A}, \mathrm{B}}$ \\
\hline White matter hyperintensities $^{\dagger}$ & $0.7(0.5)^{\mathrm{B}, \mathrm{D}}$ & $2.3(0.4)^{\mathrm{A}, \mathrm{C}}$ & $0.8(0.4)^{\mathrm{B}, \mathrm{D}}$ & $2.4(0.5)^{\mathrm{A}, \mathrm{C}}$ \\
\hline \multicolumn{5}{|l|}{ Follow-up characteristics } \\
\hline Follow-up time & $2.1(1.5)$ & $2.2(1.3)$ & $2.1(1.2)$ & $2.4(1.2)$ \\
\hline Time to progression to dementia & $1.3(0.5)^{\mathrm{B}}$ & $2.0(0.7)^{\mathrm{A}}$ & $1.7(0.7)$ & $2.1(1.2)$ \\
\hline Progression to dementia, $\mathrm{n}$ & $8(6)^{\mathrm{B}, \mathrm{C}, \mathrm{D}}$ & $9(23)^{\mathrm{A}}$ & $18(29)^{\mathrm{A}}$ & $11(38)^{\mathrm{A}}$ \\
\hline - AD-type dementia, $\mathrm{n}$ & $2(1)^{\mathrm{B}, \mathrm{C}, \mathrm{D}}$ & $7(18)^{\mathrm{A}}$ & $18(29)^{\mathrm{A}}$ & $10(35)^{\mathrm{A}}$ \\
\hline - Vascular dementia, $\mathrm{n}$ & $0(0)$ & $2(5)$ & $0(0)$ & $1(3)$ \\
\hline - Frontotemporal dementia, $\mathrm{n}$ & $4(3)$ & $0(0)$ & $0(0)$ & $0(0)$ \\
\hline - Lewy Body dementia, $\mathrm{n}$ & $1(1)$ & $0(0)$ & $0(0)$ & $0(0)$ \\
\hline - Dementia of unknown etiology, n & $1(1)$ & $0(0)$ & $0(0)$ & $0(0)$ \\
\hline
\end{tabular}

Results are mean (SD) for continuous variables or frequency (\%). Abbreviations: $A \beta=$ amyloid-beta, $A D=$ Alzheimer's disease, APOE = Apolipoprotein E, MCI= Mild Cognitive Impairment. Hypertension, obesity, diabetes and APOE $\varepsilon 4$ genotype were only available in a subgroup of the sample. ${ }^{\dagger} \mathrm{WMH}$ measured by the Fazekas scale, range $0-3 .{ }^{A} \mathrm{p}<0.05$ compared to $\mathrm{A} \beta-\mathrm{WMH}-,{ }^{\mathrm{B}} \mathrm{p}<0.05$ compared to $\mathrm{A} \beta-\mathrm{WMH}+,{ }^{\mathrm{C}} \mathrm{p}<0.05$ compared to $\mathrm{A} \beta+\mathrm{WMH}-{ }^{\mathrm{D}} \mathrm{p}<0.05$ compared to $\mathrm{A} \beta+\mathrm{WMH}+$.

\section{Neurodegeneration markers}

Table 2 shows the values and frequency of abnormal neurodegenerative markers for the $\mathrm{A} \beta / \mathrm{WMH}$ groups. Compared to the group without pathology, we found that MTA was more severe in the group with only $\mathrm{A} \beta(\mathrm{p}<0.001)$, only WMH $(\mathrm{p}<0.001)$, as well as in the mixed pathology group $(\mathrm{p}<0.001)$. The $\mathrm{A} \beta+\mathrm{WMH}+$ group had higher MTA scores, compared to the group with only WMH ( $\mathrm{p}=0.025)$ and the group with only $\mathrm{A} \beta(\mathrm{p}=0.002)$. Ttau was increased in all three groups with a form of pathology, compared to the group without pathology $(\mathrm{A} \beta-\mathrm{WMH}+\mathrm{p}<0.001 ; \mathrm{A} \beta+\mathrm{WMH}-\mathrm{p}<0.001 ; \mathrm{A} \beta+\mathrm{WMH}+\mathrm{p}=0.047)$, but this effect was influenced by baseline diagnosis $\left(\mathrm{A} \beta / \mathrm{WMH}\right.$ group ${ }^{\star}$ baseline diagnosis: $\mathrm{F}=3.20, \mathrm{p}=0.024$ ). When stratifying by diagnosis, the effect was only found in MCI subjects. There was no difference in t-tau levels between the $\mathrm{A} \beta-\mathrm{WMH}+\mathrm{A} \beta+\mathrm{WMH}-$ and the 
$\mathrm{A} \beta+\mathrm{WMH}+$ groups, regardless of baseline diagnosis. P-tau was only increased in the group with only $A \beta$ compared to $A \beta-W M H$ - group $(p<0.001)$, regardless of baseline diagnosis. The association between $\mathrm{p}$-tau and $\mathrm{A} \beta / \mathrm{WMH}$ group was influenced by APOEgenotype, as we found that the elevated $\mathrm{p}$-tau levels in the $\mathrm{A} \beta+\mathrm{WMH}$ - group where limited to APOE- $\varepsilon 4$ carriers $\left(\mathrm{A} \beta / \mathrm{WMH}\right.$ group ${ }^{\star} \mathrm{APOE}$ status: $\left.\mathrm{F}=3.72, \mathrm{p}=0.013\right)$.

Table 2: Values of neurodegenerative markers by A $\beta / \mathrm{WMH}$ groups

\begin{tabular}{cllll} 
& $\mathrm{A} \beta-\mathrm{WMH}-$ & $\mathrm{A} \beta-\mathrm{WMH}+$ & $\mathrm{A} \beta+\mathrm{WMH}-$ & $\mathrm{A} \beta+\mathrm{WMH}+$ \\
\cline { 2 - 5 } Neurodegeneration markers & $\mathrm{n}=140$ & $\mathrm{n}=39$ & $\mathrm{n}=63$ & $\mathrm{n}=29$ \\
\hline MTA score & $1.2(1.2)^{\mathrm{B}, \mathrm{C}, \mathrm{D}}$ & $2.6(1.6)^{\mathrm{A}, \mathrm{D}}$ & $2.1(1.6)^{\mathrm{A}, \mathrm{D}}$ & $3.4(1.8)^{\mathrm{A}, \mathrm{B}, \mathrm{C}}$ \\
MTA abnormal, n & $62(45)^{\mathrm{B}, \mathrm{C}, \mathrm{D}}$ & $32(82)^{\mathrm{A}}$ & $41(67)^{\mathrm{A}, \mathrm{D}}$ & $26(93)^{\mathrm{A}, \mathrm{C}}$ \\
P-tau, pg/ml & $54.5(27.7)^{\mathrm{C}}$ & $63.2(29.3)$ & $77.0(56.3)^{\mathrm{A}}$ & $65.2(38.2)$ \\
P-tau abnormal, n & $53(38)^{\mathrm{C}}$ & $22(58)$ & $45(71)^{\mathrm{A}}$ & $15(52)$ \\
T-tau, pg/ml & $314.7(202.0)^{\mathrm{B}, \mathrm{C}, \mathrm{D}}$ & $438.4(248.0)^{\mathrm{A}}$ & $499.3(413.8)^{\mathrm{A}}$ & $426.2(275.2)^{\mathrm{A}}$ \\
T-tau abnormal, n & $36(26)^{\mathrm{B}, \mathrm{C}, \mathrm{D}}$ & $20(53)^{\mathrm{A}}$ & $36(57)^{\mathrm{A}}$ & $14(48)^{\mathrm{A}}$ \\
\hline
\end{tabular}

Results are mean (SD) and number (\%). Abbreviations: $\mathrm{A} \beta=$ amyloid-beta, MTA = Medial Temporal Lobe Atrophy, $\mathrm{P}$-tau $=$ Phosphorylated tau, $\mathrm{T}$-tau $=$ Total Tau, $\mathrm{WMH}=$ White matter hyperintensities. ${ }^{\mathrm{A}} \mathrm{p}<0.05 \mathrm{com}-$ pared to $A \beta-W M H-,{ }^{B} \mathrm{p}<0.05$ compared to $A \beta-W M H+,{ }^{C} \mathrm{p}<0.05$ compared to $A \beta+W M H-,{ }^{D} p<0.05$ compared to $\mathrm{A} \beta+\mathrm{WMH}+$. All analyses were adjusted for study, baseline diagnosis and demographics.

\section{Baseline cognitive performance and cognitive decline}

In the total sample, MMSE scores did not differ between the A $\beta / W M H$ groups at baseline (Table 3, Figure 1). Baseline MMSE scores of the individuals with MCI were lower than in individuals with SCD, regardless of pathology $(\mathrm{p}<0.001)$. In subjects with $\mathrm{MCI}$, there was only a difference in baseline MMSE score between the A $\beta$-WMH- and the $\mathrm{A} \beta+\mathrm{WMH}$ - group $(\mathrm{p}=0.020)$. In subjects with SCD, there was no difference in baseline MMSE scores. In the total sample, the groups with one or both pathologies declined in MMSE score over time $(A \beta-W M H+p=0.014, A \beta+W M H-p=0.035$ and $A \beta+W M H+$ $\mathrm{p}=0.045)$, whereas scores remained stable in the $\mathrm{A} \beta-\mathrm{WMH}$ - group $(\mathrm{p}=0.793)$. The rate of decline was higher in the $\mathrm{A} \beta-\mathrm{WMH}+$ group compared to the $\mathrm{A} \beta-\mathrm{WMH}$ - group $(p=0.035)$. There were no differences in the rate of decline between the three groups with pathology. In subjects with $S C D$, the $A \beta / W M H$ groups showed no decline over time. In subjects with MCI, results were similar as results found in the total sample. Baseline delayed recall memory scores were lower in the three groups with pathology compared to the group without pathology $(A \beta-W M H+p=0.004 ; A \beta+W M H-p<0.001 ; A \beta+W M H+$ $\mathrm{p}=0.009$ ), which was not influenced by baseline diagnosis. None of the groups showed significant decline over time. TMT-B scores did not differ at baseline between the groups and did not change during follow-up (Table 3, Figure 1). APOE genotype did not influence any of the baseline nor longitudinal associations. 
Cerebrovascular and amyloid pathology in the pre-dementia stages

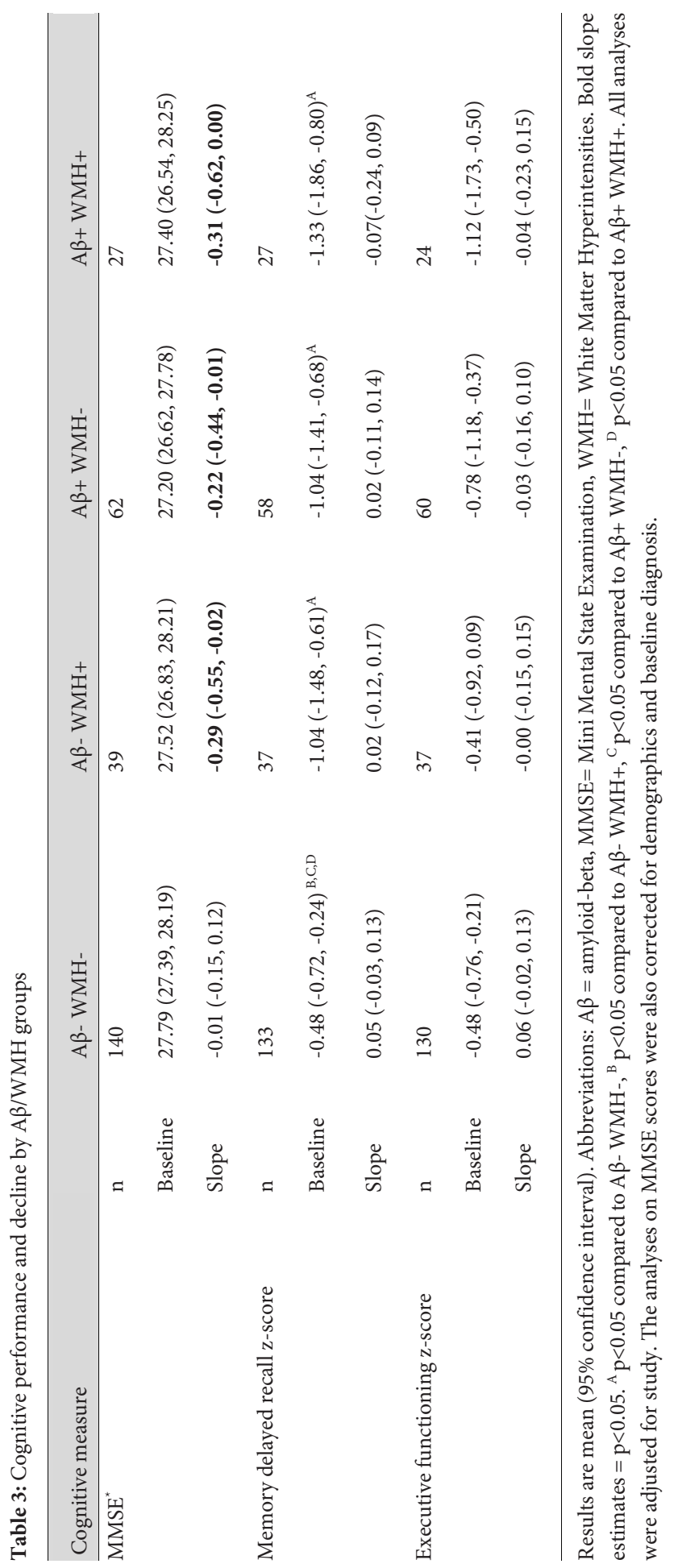




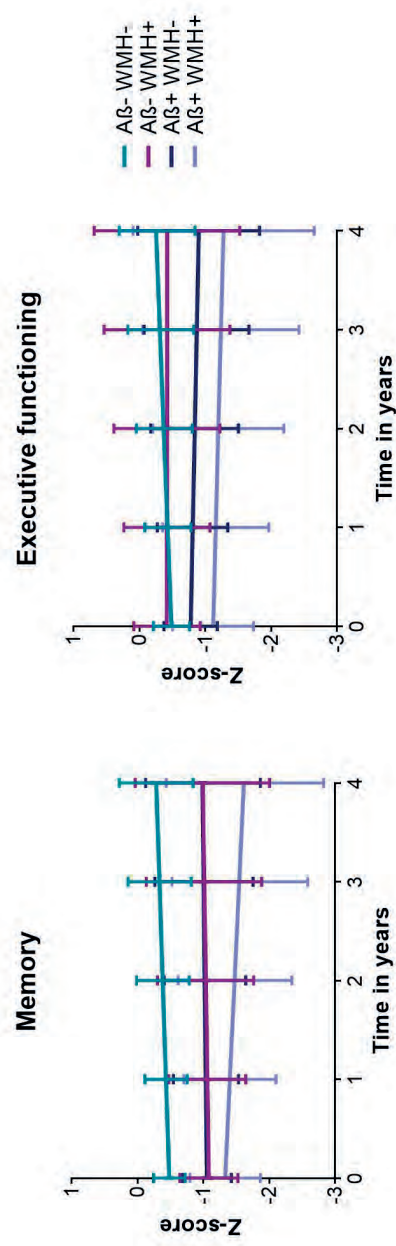

范

है हี

品完

元

:

品苛

就

$\sum^{1} \underbrace{\infty}_{0}$

in

要是

공

ก. $\Xi$

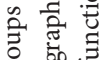

b

声 $气$

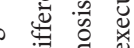

๑

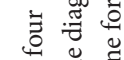

要严

范茟

पु चै

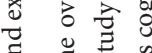

त $\exists$ की

घั0

घ

हี

पे

8 तै है

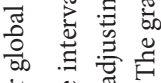

苍苛苛

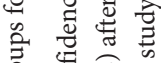

के हैं

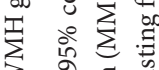

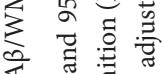

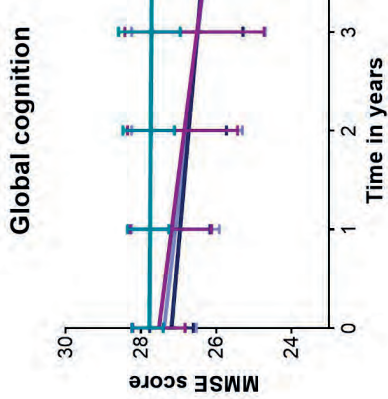

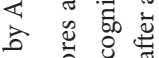

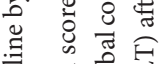

चี

घ

学岂

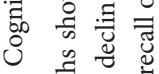

$\ddot{\nabla}$ 离.

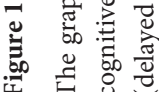




\section{Progression to dementia}

Table 4 and Figure 2 show the risk of progression to dementia for the A $\beta / \mathrm{WMH}$ groups. Compared to the group without pathology, the groups with a form of pathology have an increased risk of progressing to dementia $(\mathrm{A} \beta-\mathrm{WMH}+\mathrm{HR}: 3.25, \mathrm{p}=0.021, \mathrm{~A} \beta+\mathrm{WMH}-$ HR: 4.89, $\mathrm{p}<0.001, \mathrm{~A} \beta+\mathrm{WMH}+\mathrm{HR}: 3.00, \mathrm{p}=0.036)$, but this was influenced by baseline diagnosis $\left(\mathrm{A} \beta / \mathrm{WMH}\right.$ group ${ }^{*}$ baseline diagnosis: $\left.\mathrm{HR}=2.89 ; \mathrm{p}=0.007\right)$ as the effect was mainly attributable to MCI subjects (Figure 2). There was no difference in progression rates between the groups with isolated or co-existing $\mathrm{A} \beta / \mathrm{WMH}$ pathology, when analyzing the total sample or only MCI subjects. Results were similar when using progression to AD-type dementia as outcome.

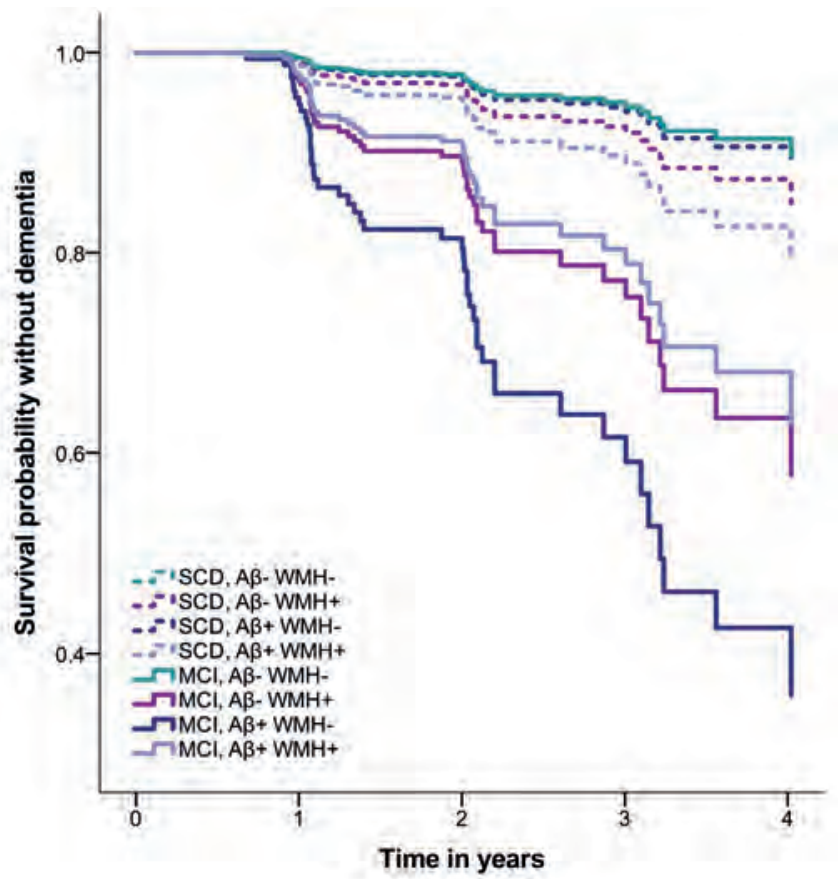

Figure 2. Risk of progression to dementia over time for $\mathrm{A} \beta / \mathrm{WMH}$ groups by baseline diagnosis.

The graph shows the probability of surviving without dementia during a four-year follow-up period for the four $\mathrm{A} \beta / \mathrm{WMH}$ groups after adjusting for demographics, study stratified by baseline diagnosis.

\section{Post-hoc analyses}

Since both $\mathrm{A} \beta$ and WMH were associated with MTA, we tested the interaction between the two pathologies on MTA. General linear model analyses showed no interaction between $\mathrm{A} \beta$ and $\mathrm{WMH}$ on MTA, using dichotomous variables created with cut-off points $(\mathrm{p}=0.770)$ or continuous variables $(\mathrm{p}=0.631)$. 
Table 4: Risk of progression to dementia for $\mathrm{A} \beta / \mathrm{WMH}$ groups

\begin{tabular}{|c|c|c|c|c|}
\hline \multirow{2}{*}{$\frac{\text { Groups }}{\mathrm{A} \beta-\mathrm{WMH}-}$} & \multirow{2}{*}{$\begin{array}{l}\text { Hazard ratio } \\
\text { Reference }\end{array}$} & \multirow{2}{*}{$\begin{array}{l}95 \% \mathrm{CI} \\
\text { Reference }\end{array}$} & \multicolumn{2}{|c|}{ P-value comparisons } \\
\hline & & & $\mathrm{A} \beta-\mathrm{WMH}+$ & 0.021 \\
\hline & & & $\mathrm{A} \beta+\mathrm{WMH}-$ & $<0.001$ \\
\hline & & & $\mathrm{A} \beta+\mathrm{WMH}+$ & 0.036 \\
\hline \multirow[t]{3}{*}{$\mathrm{A} \beta-\mathrm{WMH}+$} & 3.30 & $1.21-8.98$ & $\mathrm{~A} \beta-\mathrm{WMH}-$ & 0.021 \\
\hline & & & $\mathrm{A} \beta+\mathrm{WMH}-$ & 0.358 \\
\hline & & & $\mathrm{A} \beta+\mathrm{WMH}+$ & 0.868 \\
\hline \multirow[t]{3}{*}{$\mathrm{A} \beta+\mathrm{WMH}-$} & 4.84 & $2.03-11.51$ & $\mathrm{~A} \beta-\mathrm{WMH}-$ & $<0.001$ \\
\hline & & & $\mathrm{A} \beta-\mathrm{WMH}+$ & 0.358 \\
\hline & & & $\mathrm{A} \beta+\mathrm{WMH}+$ & 0.294 \\
\hline \multirow[t]{3}{*}{$\mathrm{A} \beta+\mathrm{WMH}+$} & 3.02 & $1.08-8.43$ & $\mathrm{~A} \beta-\mathrm{WMH}-$ & 0.036 \\
\hline & & & $\mathrm{A} \beta-\mathrm{WMH}+$ & 0.868 \\
\hline & & & $\mathrm{A} \beta+\mathrm{WMH}-$ & 0.294 \\
\hline
\end{tabular}

Abbreviations: $\mathrm{A} \beta=$ amyloid-beta, $\mathrm{CI}=$ Confidence Interval, $\mathrm{WMH}=$ White Matter Hyperintensities. Analyses are adjusted for demographics, study and baseline diagnosis.

We repeated the main analyses after exclusion of subjects with CSF A $\beta$ 1-42 values $10 \%$ around the cut-off. Results remained similar after this exclusion. Results were also comparable after exclusion of the four subjects in the $A \beta-W M H$ - group who progressed to frontotemporal dementia (FTD) at follow-up, and when repeating the analyses using A $\beta$ and tau for classification of AD profiles, instead of only $\mathrm{A} \beta$.

We conducted age-sensitivity analyses in which we age-matched the A $\beta / \mathrm{WMH}$ groups by selecting only individuals between 64 and 79 years of age. Most results were similar to the original results. Results that were different are shown in Supplemental Table 1. Associations that showed a similar direction but no longer reached significance because of a reduction in sample size (p-values between 0.05 and 0.09), were considered unchanged.

Observed power calculations were done for the main analyses. For the comparisons of neurodegenerative markers (Table 2), the observed power ranged from $0.71-0.99$. For the comparisons in cognitive performance and decline (Table 3), the observed baseline power ranged from $0.37-0.67$, and from $0.34-0.61$ for the slopes. Regarding the comparisons in progression to dementia (Table 4), the observed power was 0.66 .

\section{DISCUSSION}

We investigated the relation of $\mathrm{A} \beta$ and CVD pathology with markers for neurodegeneration and cognitive decline. We found that the neurodegeneration markers t-tau in CSF and MTA on MRI, were associated with both $A \beta$ and CVD, and that there was an additive 
association of the two pathologies on MTA. Decline of global cognition scores during follow-up was seen in both $\mathrm{A} \beta$ and $\mathrm{CVD}$, but there was no additive or synergistic effect.

\section{Medial temporal lobe atrophy}

The association between AD pathology and MTA has been well characterized in literature, as the first neuropathological changes underlying $\mathrm{AD}$ are thought to occur in the medial temporal lobe. ${ }^{37}$ The relationship between MTA and CVD however is still somewhat controversial. Some studies have found that CVD was associated with MTA, ${ }^{12,15,38}$ while other did not find this relationship. ${ }^{7,13}$ Our results support an association between CVD and MTA, although we measured only one aspect of CVD, i.e. WMH. Interestingly, we found that MTA was most severe in the group with mixed $A \beta / \mathrm{WMH}$ pathology, compared to the groups with a single form of pathology. In post-hoc analyses we found no interaction between $\mathrm{A} \beta$ and $\mathrm{WMH}$ on MTA and therefore we conclude that WMH and amyloid are independent determinants to MTA severity and when both present, their effects are additive.

Tau

Increased CSF t-tau was associated with both WMH and $\mathrm{A} \beta$ pathology, which is consistent with previous studies where $\mathrm{t}$-tau was considered a measure of neuronal damage. ${ }^{14,39}$ Our finding that also in patients with WMH and no A $\beta$ pathology t-tau levels are elevated, contradicts with a previous study that concluded that elevated $\mathrm{t}$-tau levels in patients with vascular damage could be the result of co-existing A $\beta$ pathology. ${ }^{40}$ That elevated t-tau levels in the groups with one or both pathologies were only found in subjects with MCI, is in line with previous work that strongly related tau to cognitive dysfunction. ${ }^{41} \mathrm{P}$-tau was only significantly increased in the $\mathrm{A} \beta+\mathrm{WMH}$ - group and only slightly increased in the $\mathrm{A} \beta+\mathrm{WMH}+$ group, supporting that this could be a specific biomarker for AD. ${ }^{39}$

\section{Amyloid-beta}

We found similar levels of $A \beta$ for the group with only amyloid pathology and the group with mixed $A \beta / W M H$ pathology. This was in contrast to our expectations based on literature, as we expected the group with mixed pathology to have a lower amyloid load (i.e. higher A $\beta$ 1-42 levels). ${ }^{5,6}$ Possibly the cognitive status of the investigated population (i.e. individuals with SCD or MCI vs. cognitively normal individuals) might play a role. In particular, in combination with the method of measuring amyloid load (by CSF or amyloid-PET), as a study comparing these two methods showed that disconcordance was de- 
pendent on disease stage. ${ }^{42}$ Further studies should determine the associations of both factors with amyloid load in mixed $\mathrm{AD} / \mathrm{CVD}$ patients. The suggestion of expanding the recently proposed "A/T/N" classification system with a vascular component would be valuable in addressing these and other research questions. ${ }^{43}$

\section{Cognitive performance and decline}

The decrease in performance over time in global cognition was similar for the three groups with pathology, indicating that $\mathrm{WMH}$ as well as $\mathrm{A} \beta$ pathology are drivers of cognitive decline. This in line with findings from a previous study in cognitively normal individuals that also found that both pathologies contribute to cognitive decline. ${ }^{9}$ However, in contrast to this previous study, we did not find any differences in cognitive trajectories between individuals with only $\mathrm{A} \beta$ pathology and those with mixed $\mathrm{A} \beta / \mathrm{WMH}$ pathology. This may relate to the fact that we included subjects with SCD and MCI instead of cognitively normal subjects or to the type of cognitive measures used. Also, risk of progression to dementia during follow-up did not differ between $\mathrm{WMH}$ and $\mathrm{A} \beta$, and having both pathologies simultaneously did not increase the risk any further.

\section{Strengths and limitations}

This study has several limitations. First, using WMH as a marker of CVD can be seen as a limitation as we did not take other forms of CVD as lacunar infarcts or cortical microbleeds into account. Although only using WMH reflects a method of defining vascular damage frequently used in clinical practice, ${ }^{44}$ this makes our findings less generalizable to CVD in general. Also, WMH are heterogeneous in their etiology and pathophysiology, and the underlying mechanisms causing WMH are not yet completely understood. ${ }^{45}$ However, in an aging population as studied in the current study, WMH are mostly considered a consequence of cerebral vascular damage. ${ }^{46,47}$ Second, our follow-up length ranged from one to four years, which might have been too short to detect differences in cognitive decline in non-demented individuals. Third, our sample was derived from different studies, which might have led to variability in the data, despite adjustment for study in all of the analyses. However, our multi-study design makes our findings more generalizable to other memory clinic settings. Fourth, a methodological consideration of this study was that our results were based on both subjects with SCD and MCI. Although we did examine the influence of the baseline diagnosis in all analyses and when needed adjusted for this and reported the differences, the smaller sample sizes when analyzing per diagnosis, could have influenced the results. The smaller sample sizes in general could reflect a lack of statistical power, and therefore our results should be interpreted with caution and validated in future studies. Although observed power calculations should be interpreted with caution, ${ }^{48}$ we would recommend future studies to make group sizes more 
balanced and to include a larger number of complete follow-up visits, in particular for outcome measures with smaller effect-sizes (e.g. executive functioning measures). The major strengths of this study were the longitudinal set-up, the reflection of clinical practice and the availability of different neurodegeneration markers to provide novel insights into the role of neurodegeneration in relation to $\mathrm{AD}$ and CVD.

\section{Conclusions}

The findings of the current study may have implications for the diagnosis and prognosis of memory clinic patients, but also for future scientific research. For clinicians, it is important to realize that MTA on MRI, and elevated t-tau values in CSF may reflect underlying $\mathrm{AD}$ as well as CVD pathology, and that the effects of $\mathrm{A} \beta$ and $\mathrm{WMH}$ on MTA could be additive. Based on the data from the current study we conclude that the short-term cognitive prognosis of SCD or MCI patients with mixed amyloid/WMH pathology may be similar to patients with solely amyloid or WMH pathology. Future research with longer follow-up and a larger sample size is needed to confirm these findings and determine whether this is also the case when focusing on long-term prognosis. 


\section{REFERENCES}

1. Schneider JA, Aggarwal NT, Barnes L, Boyle P, Bennett DA. The neuropathology of older persons with and without dementia from community versus clinic cohorts. Journal of Alzheimer's disease : JAD. 2009;18(3):691-701.

2. Attems J, Jellinger KA. The overlap between vascular disease and Alzheimer's disease--lessons from pathology. BMC Med. 2014;12:206.

3. Heyman A, Fillenbaum GG, Welsh-Bohmer KA, et al. Cerebral infarcts in patients with autopsy-proven Alzheimer's disease: CERAD, part XVIII. Consortium to Establish a Registry for Alzheimer's Disease. Neurology. 1998;51(1):159-162.

4. Zea-Sevilla MA, Fernandez-Blazquez MA, Calero M, Bermejo-Velasco P, Rabano A. Combined Alzheimer's disease and cerebrovascular staging explains advanced dementia cognition. Alzheimer's \& dementia: the journal of the Alzheimer's Association. 2015;11(11):1358-1366.

5. Jack CR, Jr., Wiste HJ, Vemuri P, et al. Brain beta-amyloid measures and magnetic resonance imaging atrophy both predict time-to-progression from mild cognitive impairment to Alzheimer's disease. Brain : a journal of neurology. 2010;133(11):3336-3348.

6. Jellinger KA. Understanding the pathology of vascular cognitive impairment. Journal of the neurological sciences. 2005;229-230:57-63.

7. Vemuri P, Knopman DS. The role of cerebrovascular disease when there is concomitant Alzheimer disease. Biochimica et biophysica acta. 2016;1862(5):952-956.

8. Zekry D, Duyckaerts C, Moulias R, et al. Degenerative and vascular lesions of the brain have synergistic effects in dementia of the elderly. Acta neuropathologica. 2002;103(5):481-487.

9. Vemuri P, Lesnick TG, Przybelski SA, et al. Vascular and amyloid pathologies are independent predictors of cognitive decline in normal elderly. Brain : a journal of neurology. 2015;138(Pt 3):761-771.

10. Nebes RD, Snitz BE, Cohen AD, et al. Cognitive aging in persons with minimal amyloid-beta and white matter hyperintensities. Neuropsychologia. 2013;51(11):2202-2209.

11. Ye BS, Seo SW, Kim GH, et al. Amyloid burden, cerebrovascular disease, brain atrophy, and cognition in cognitively impaired patients. Alzheimer's of dementia : the journal of the Alzheimer's Association. 2015;11(5):494-503.e493.

12. Bastos-Leite AJ, van der Flier WM, van Straaten EC, Staekenborg SS, Scheltens P, Barkhof F. The contribution of medial temporal lobe atrophy and vascular pathology to cognitive impairment in vascular dementia. Stroke; a journal of cerebral circulation. 2007;38(12):3182-3185.

13. Lo RY, Jagust WJ, Alzheimer's Disease Neuroimaging I. Vascular burden and Alzheimer disease pathologic progression. Neurology. 2012;79(13):1349-1355.

14. Hesse C, Rosengren L, Andreasen N, et al. Transient increase in total tau but not phospho-tau in human cerebrospinal fluid after acute stroke. Neuroscience letters. 2001;297(3):187-190.

15. Freeze WM, Jacobs HI, Gronenschild EH, et al. White Matter Hyperintensities Potentiate Hippocampal Volume Reduction in Non-Demented Older Individuals with Abnormal Amyloid-beta. Journal of Alzheimer's disease : JAD. 2016.

16. Handels RL, Aalten P, Wolfs CA, et al. Diagnostic and economic evaluation of new biomarkers for Alzheimer's disease: the research protocol of a prospective cohort study. BMC Neurol. 2012;12:72.

17. Visser PJ, Verhey FR, Boada M, et al. Development of screening guidelines and clinical criteria for predementia Alzheimer's disease. The DESCRIPA Study. Neuroepidemiology. 2008;30(4):254-265.

18. Lezak MD, Howieson DB, Loring DW. Neuropsychological Assessment. Oxford, UK: Oxford University Press 2004.

19. Reitan R. Trail-Making Test. Arizona: Reitan Neuropsychology Laboratory 1979.

20. Petersen RC. Mild cognitive impairment as a diagnostic entity. Journal of internal medicine. 2004;256(3):183-194. 
21. APA. Diagnostic and Statistical Manual of Mental Disorders: DSM-IV. Washington, DC: American Psychiatric Association; 1994.

22. McKhann G, Drachman D, Folstein M, Katzman R, Price D, Stadlan EM. Clinical diagnosis of Alzheimer's disease: report of the NINCDS-ADRDA Work Group under the auspices of Department of Health and Human Services Task Force on Alzheimer's Disease. Neurology. 1984;34(7):939-944.

23. Roman GC, Tatemichi TK, Erkinjuntti T, et al. Vascular dementia: diagnostic criteria for research studies. Report of the NINDS-AIREN International Workshop. Neurology. 1993;43(2):250-260.

24. Neary D, Snowden JS, Gustafson L, et al. Frontotemporal lobar degeneration: a consensus on clinical diagnostic criteria. Neurology. 1998;51(6):1546-1554.

25. McKeith IG, Galasko D, Kosaka K, et al. Consensus guidelines for the clinical and pathologic diagnosis of dementia with Lewy bodies (DLB): report of the consortium on DLB international workshop. Neurology. 1996;47(5):1113-1124.

26. Vos SJ, Visser PJ, Verhey F, et al. Variability of CSF Alzheimer's disease biomarkers: implications for clinical practice. PloS one. 2014;9(6):e100784.

27. de Jong D, Kremer BP, Olde Rikkert MG, Verbeek MM. Current state and future directions of neurochemical biomarkers for Alzheimer's disease. Clinical chemistry and laboratory medicine : CCLM / FESCC. 2007;45(11):1421-1434.

28. Mulder C, Verwey NA, van der Flier WM, et al. Amyloid-beta(1-42), total tau, and phosphorylated tau as cerebrospinal fluid biomarkers for the diagnosis of Alzheimer disease. Clinical chemistry. 2010;56(2):248253.

29. Aalten P, Ramakers IH, Biessels GJ, et al. The Dutch Parelsnoer Institute--Neurodegenerative diseases; methods, design and baseline results. BMC Neurol. 2014;14:254.

30. Scheltens P, Leys D, Barkhof F, et al. Atrophy of medial temporal lobes on MRI in "probable" Alzheimer's disease and normal ageing: diagnostic value and neuropsychological correlates. Journal of neurology, neurosurgery, and psychiatry. 1992;55(10):967-972.

31. Korf ES, Wahlund LO, Visser PJ, Scheltens P. Medial temporal lobe atrophy on MRI predicts dementia in patients with mild cognitive impairment. Neurology. 2004;63(1):94-100.

32. Wahlund LO, Barkhof F, Fazekas F, et al. A new rating scale for age-related white matter changes applicable to MRI and CT. Stroke; a journal of cerebral circulation. 2001;32(6):1318-1322.

33. Fazekas F, Chawluk JB, Alavi A, Hurtig HI, Zimmerman RA. MR signal abnormalities at $1.5 \mathrm{~T}$ in Alzheimer's dementia and normal aging. AJR American journal of roentgenology. 1987;149(2):351-356.

34. Wahlund LO, Westman E, van Westen D, et al. Imaging biomarkers of dementia: recommended visual rating scales with teaching cases. Insights Imaging. 2017;8(1):79-90.

35. Carotenuto A, Rea R, Colucci L, et al. Late and early onset dementia: what is the role of vascular factors? A retrospective study. Journal of the neurological sciences. 2012;322(1-2):170-175.

36. Fazekas F, Barkhof F, Wahlund LO, et al. CT and MRI rating of white matter lesions. Cerebrovasc Dis. 2002;13 Suppl 2:31-36.

37. Braak H, Braak E. Neuropathological stageing of Alzheimer-related changes. Acta neuropathologica. 1991;82(4):239-259.

38. Akinyemi RO, Firbank M, Ogbole GI, et al. Medial temporal lobe atrophy, white matter hyperintensities and cognitive impairment among Nigerian African stroke survivors. BMC research notes. 2015;8:625.

39. Blennow K, Hampel H. CSF markers for incipient Alzheimer's disease. The Lancet Neurology. 2003;2(10):605-613.

40. Kurz A, Riemenschneider M, Wallin A. Potential biological markers for cerebrovascular disease. International psychogeriatrics / IPA. 2003;15 Suppl 1:89-97.

41. Nelson PT, Alafuzoff I, Bigio EH, et al. Correlation of Alzheimer disease neuropathologic changes with cognitive status: a review of the literature. Journal of neuropathology and experimental neurology. 2012;71(5):362-381. 
42. Mattsson N, Insel PS, Donohue M, et al. Independent information from cerebrospinal fluid amyloid-beta and florbetapir imaging in Alzheimer's disease. Brain : a journal of neurology. 2015;138(Pt 3):772-783.

43. Jack CR, Jr., Bennett DA, Blennow K, et al. A/T/N: An unbiased descriptive classification scheme for Alzheimer disease biomarkers. Neurology. 2016;87(5):539-547.

44. Debette S, Markus HS. The clinical importance of white matter hyperintensities on brain magnetic resonance imaging: systematic review and meta-analysis. Bmj. 2010;341:c3666.

45. Gouw AA, Seewann A, van der Flier WM, et al. Heterogeneity of small vessel disease: a systematic review of MRI and histopathology correlations. Journal of neurology, neurosurgery, and psychiatry. 2011;82(2):126-135.

46. Prins ND, Scheltens P. White matter hyperintensities, cognitive impairment and dementia: an update. Nat Rev Neurol. 2015;11(3):157-165.

47. Wardlaw JM, Valdes Hernandez MC, Munoz-Maniega S. What are white matter hyperintensities made of? Relevance to vascular cognitive impairment. Journal of the American Heart Association. 2015;4(6):001140.

48. Hoenig JM, Heisey DM. The abuse of power: the pervasive fallacy of power calculations for data analysis. The American Statistician. 2001;55(1):19-24. 


\section{SUPPLEMENTAL DATA}

Supplemental Text 1. Scan parameters and MRI protocols used at each centre.

\section{DESCRIPA}

\section{CENTER HUDDINGE - STOCKHOLM}

Siemens Avanto 1.5 T, 21 slices, FOV $220 \mathrm{~mm}$, FOV phase 87.1, distance factor 30, phase R $>$ L, slice thickness $5.0 \mathrm{~mm}$, TE: $96 \mathrm{~ms}$, TR: $4000 \mathrm{~ms}$, flip angle $150^{\circ}$, number of averages 1 .

Siemens Symphony 1.5 T, 21 slices, FOV 220 mm, FOV phase 75.0, distance factor 30, phase R>L, slice thickness $5.0 \mathrm{~mm}$, TE: $99 \mathrm{~ms}$, TR: $4100 \mathrm{~ms}$, flip angle $150^{\circ}$, number of averages 2 .

\section{CENTER KUOPIO}

Siemens Vision 1.5 T, T1 3D-scan, MPRAGE OBL; COR>TRA, FOV 250, mat 256x256, 128 slices, TR $9.7 \mathrm{~ms}$, TE $4 \mathrm{~ms}$, Slice th $2.0 \mathrm{~mm}$, no slice gap, flip angle $12^{\circ}$

\section{CENTER MALMO}

Siemens Sonata 1.5 T, MPRAGE + lmpr-cor, 144 slices, FOV $250 \mathrm{~mm}$, phase R>L, TR 1970, TE 3.93, distance factor 50 , slice thickness $1.5 \mathrm{~mm}$, flip angle $15^{\circ}$.

\section{CENTER MUNICH}

Siemens Magnetom Vision; 1.5 T; MPRAGE; Slice thickness 1.05 mm, TR 11.4, TE 4.4, TI 300; FOV $256^{\star} 256$; Flip angle $8^{\circ}$; number of averages 1 .

\section{CENTER THESSALONIKI}

Siemens Expert Plus unit 1.0 T, 3D-MPR: 15 (TR) ,7 TE ,8 FLIP ANG., 250 (Slabth), 1,49Ef thick, 168 Partitions, 250 FOV,256x192 Matrix, 1 Aquis. ,ACQ TIME 10,21 min

\section{CENTER MAASTRICHT}

Philip NT, 1.5 T Gyroscan: T1-weighted images obtained in the coronal plane using a 3D-gradient fast field echo (FFE) sequence. $\mathrm{TR}=35 \mathrm{~ms}$, $\mathrm{TE}=7 \mathrm{~ms}, \mathrm{FA}=35, \mathrm{FOV}=240 \mathrm{~mm}$, slice thickness = $1.5 \mathrm{~mm}$, matrix size $=256 \times 256$, voxelsize $=0.94 \mathrm{~mm} \times 0.94 \mathrm{~mm} \times 1.5 \mathrm{~mm}$.

\section{CENTER MANNHEIM}

Siemens Medical solution Magnetom, Vision plus 1.5 Tesla: T1 MPR 30, TR=11.4 ms, TE=4.4 ms, flipangle $=15, F o V=256 \mathrm{~mm}$, format $8 / 8$, slices $=162$, no gap.

\section{CENTER VUMC}

Siemens Magnetom Impact Expert 1.0 T, 3D scan, 168 slices, FOV 250 mm, matrix $256 \times 256$; slice thickness $1.5 \mathrm{~mm}$, TE: $7 \mathrm{~ms}$, TR: $15 \mathrm{~ms}$, TI $300 \mathrm{~ms}$, flip angle $15^{\circ}$. 
Chapter 6

LeARN

\section{CENTER LEIDEN}

Philips Achieva, 3.0 T, 3D scan, 180 slices; matrix: 288 x 288; voxel size: 0.7778 x 0.7778 x 1.00; TE: $4.6 \mathrm{~ms}$; TR: $9.8 \mathrm{~ms}$; flip angle: $8^{\circ}$.

\section{CENTER NIJMEGEN}

Siemens TrioTim 3.0 T, 3D scan; 192 slices; matrix: 256 x 256; voxel size: 1.00 x 1.00 x 1.00; TE: 4.7 ms; TR: $2300 \mathrm{~ms}$; TI: $1100 \mathrm{~ms}$; flip angle: $90^{\circ}$.

\section{CENTER VUMC}

GE Signa HDxt 3.0 T; 3D-scan; 176 slices; matrix: 256 x 256; voxel size: 0.94 x 0.94 x 1.00; TE: 3.0 ms; TR: $7.8 \mathrm{~ms}$; TI: $450 \mathrm{~ms}$; flip angle: $90^{\circ}$

\section{CENTER MAASTRICHT + additional subjects from BBACL}

Philips Achieva 3.0 T; 3D scan; 180 slices; 240 x 240; voxel size: 1.00 x 1.00 x 1.00; TE: 3.7 ms; TR: $8.2 \mathrm{~ms}$; flip angle: $8^{\circ}$. 
Supplemental Table 1. Additional results in age-matched groups

\begin{tabular}{lllll} 
& $\mathrm{A} \beta-\mathrm{WMH}-$ & $\mathrm{A} \beta-\mathrm{WMH}+$ & $\mathrm{A} \beta+\mathrm{WMH}-$ & $\mathrm{A} \beta+\mathrm{WMH}+$ \\
\cline { 2 - 5 } & $\mathrm{n}=52$ & $\mathrm{n}=26$ & $\mathrm{n}=43$ & $\mathrm{n}=22$ \\
\hline $\begin{array}{l}\text { Age } \\
\text { Neurodegeneration markers }\end{array}$ & $70.4(4.3)$ & $70.5(3.8)$ & $72.8(3.9)$ \\
MTA score & $1.6(1.4)^{\mathrm{B}, \mathrm{D}}$ & $2.6(1.5)^{\mathrm{A}}$ & $2.2(1.7)$ & $2.9(1.6)^{\mathrm{A}}$ \\
$\begin{array}{l}\text { MTA abnormal, } \mathrm{n} \\
\text { Mognition }\end{array}$ & $31(62 \%)^{\mathrm{D}}$ & $21(81 \%)$ & $22(67 \%)^{\mathrm{D}}$ & $19(91 \%)^{\mathrm{A}, \mathrm{C}}$ \\
MMSE - Slope & $-0.10(-0.36,0.16)^{\mathrm{C}}$ & $-0.35(-0.73,0.03)$ & $-0.65(-1.03,-0.26)^{\mathrm{A}}$ & $-0.45(-0.87,-0.03)$ \\
Memory - Slope & $0.09(-0.02,0.20)^{\mathrm{D}}$ & $0.06(-0.10,0.23)$ & $-0.11(-0.29,0.06)$ & $-0.12(-0.29,0.06)^{\mathrm{A}}$ \\
\hline
\end{tabular}

Results are mean (SD or 95\% CI) or frequency (\%). Abbreviations: A $\beta=$ amyloid-beta, MMSE= Mini Mental State Examination, $\mathrm{WMH}=$ White Matter Hyperintensities. Bold slope estimates $=\mathrm{p}<0.05$. ${ }^{\mathrm{A}} \mathrm{p}<0.05$ compared to $\mathrm{A} \beta$ - WMH-, ${ }^{\mathrm{B}} \mathrm{p}<0.05$ compared to $\mathrm{A} \beta-\mathrm{WMH}+,{ }^{\mathrm{C}} \mathrm{p}<0.05$ compared to $\mathrm{A} \beta+\mathrm{WMH}-,{ }^{\mathrm{D}} \mathrm{p}<0.05$ compared to $\mathrm{A} \beta+\mathrm{WMH}+$. All analyses were adjusted for study. The analyses on MMSE scores were also corrected for demographics and baseline diagnosis. 



\section{Part}

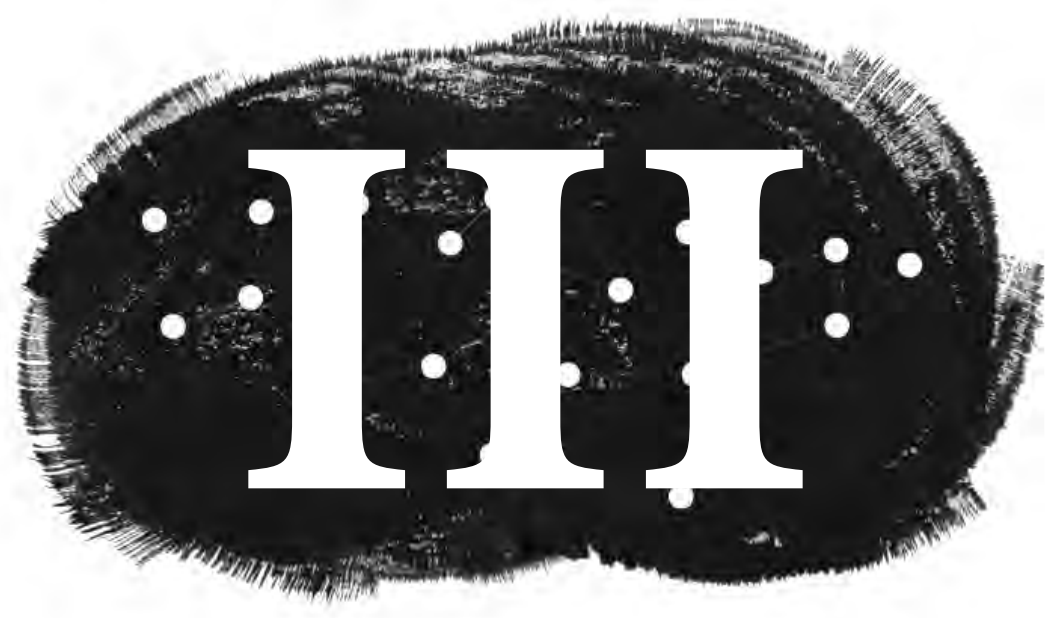

Established and novel cerebrospinal fluid biomarkers 


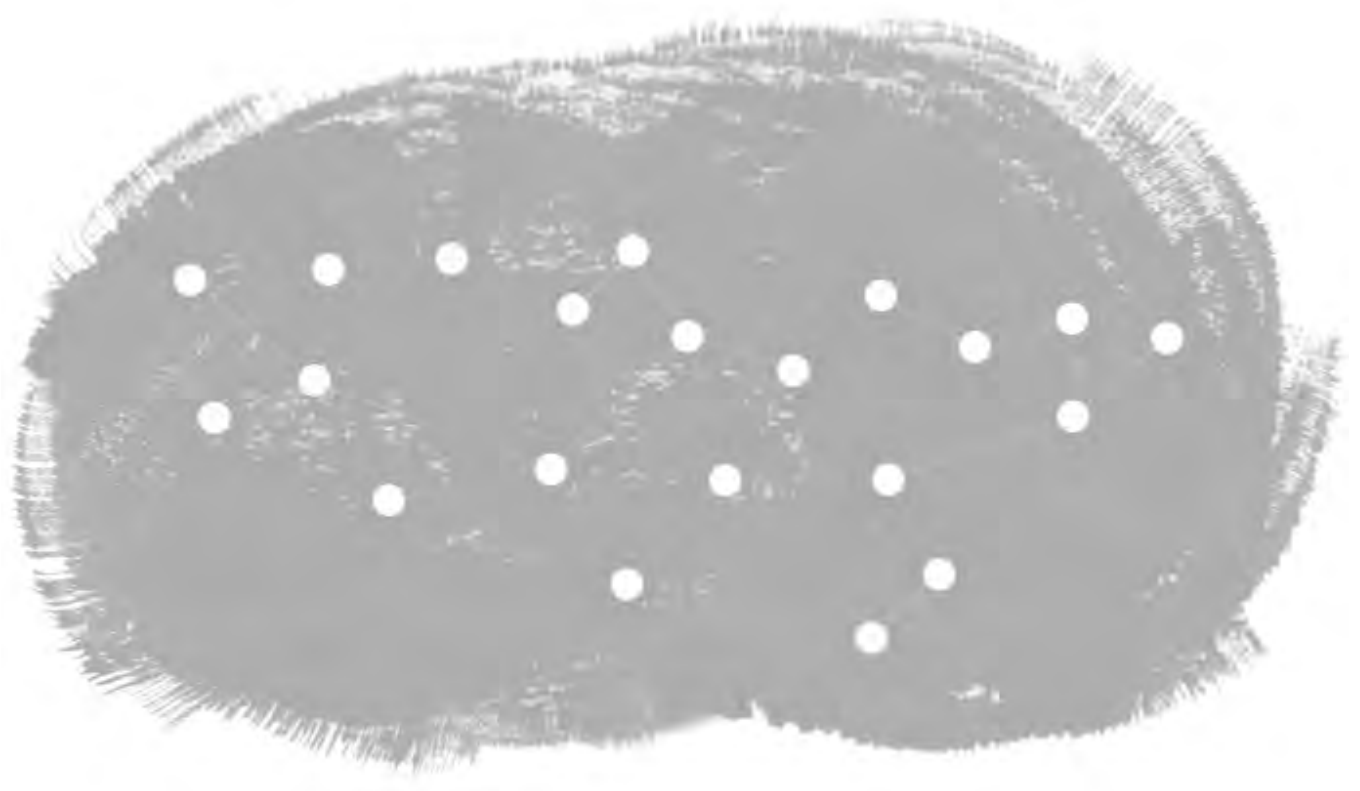




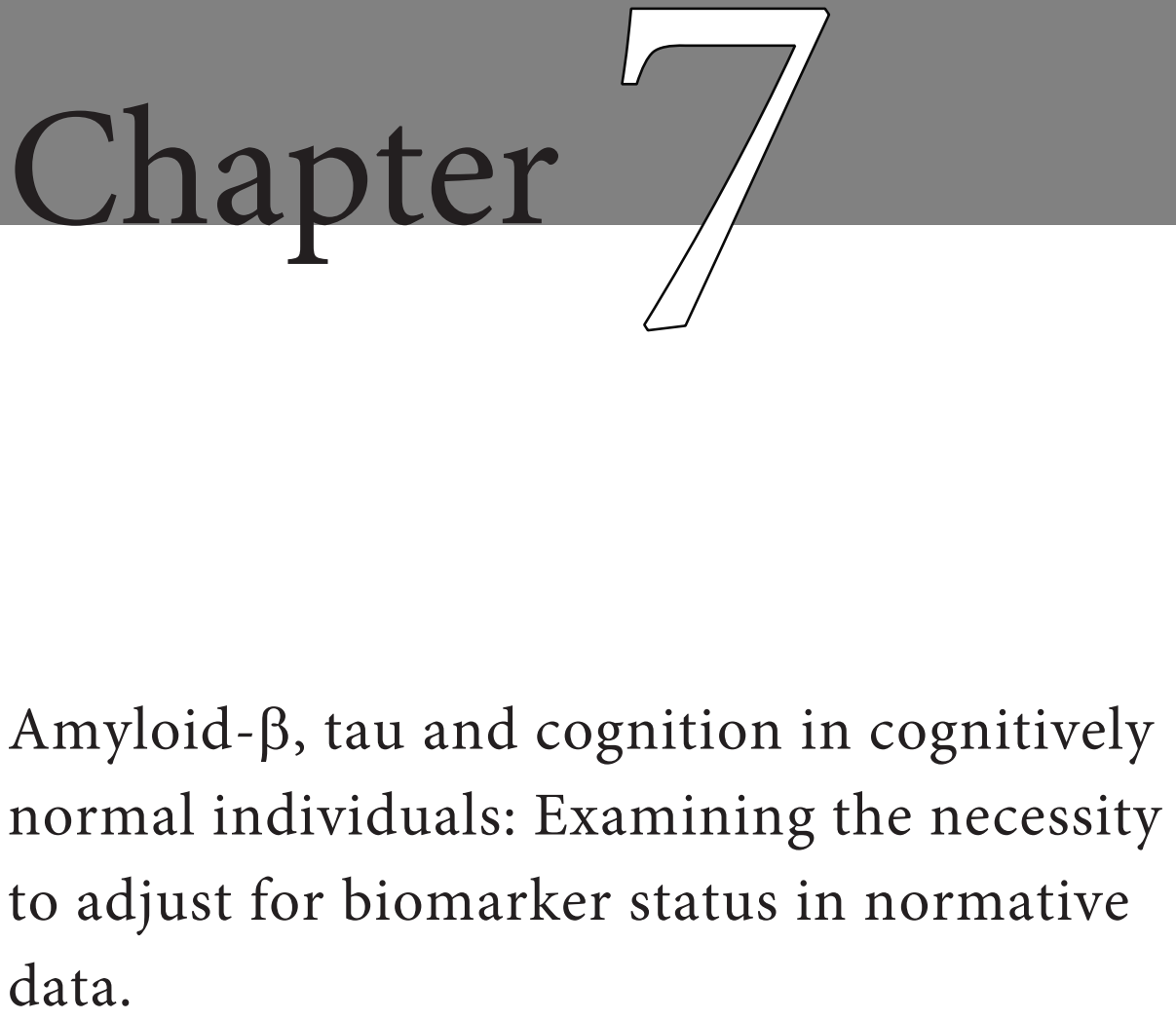

Frontiers in Aging Neuroscience, 2018, 10 (193)

Isabelle Bos, Stephanie J.B. Vos, Willemijn J. Jansen, Rik Vandenberghe, Silvy Gabel, Ainara Estanga, Mirian Ecay-Torres, Jori Tomassen, Anouk den Braber, Alberto Lleó, Isabel Sala, Anders Wallin, Petronella Kettunen, José Luis Molinuevo, Lorena Rami, Gaël Chetelat, Vincent de la Sayette, Magda Tsolaki, Yvonne Freund-Levi, Peter Johannsen, the Alzheimer's Disease Neuroimaging Initiative, Gerald Novak, Inez Ramakers, Frans Verhey and Pieter Jelle Visser 


\section{ABSTRACT}

Background: Norms for cognitive tests are usually based on the performance of cognitively normal individuals. However, some of these individuals may already have Alzheimer's disease $(\mathrm{AD})$ pathology, which could influence their cognitive test performance. We investigated whether amyloid- $\beta(A \beta)$ and tau affected cognition in cognitively normal individuals and whether norms based on biomarker negative individuals might improve early detection of dementia by cognitive tests.

Methods: We used data from 907 cognitively normal individuals from 7 cohorts of the European Medical Information Framework for AD (EMIF-AD) project and from the ADNI study. Inclusion criteria were age older than 40, no cognitive impairment, baseline data available on A $\beta$ measurement (in CSF or PET) and neuropsychological assessment. Linear mixed models were used to assess the influence of $A \beta$ and tau on the Rey Auditory Verbal Learning Test (RAVLT), Verbal Fluency and Trail Making Test (TMT) part A and B. For tests that were associated with AD pathology, we calculated norms in biomarker negative individuals. These novel norms were validated in the independent Maastricht ACL cohort $(n=1070)$.

Results: $A \beta$ pathology was associated with lower scores on the AVLT Immediate and Delayed recall, TMT part A and B, whereas we found no influence of tau. For these four tests, we calculated norms in individuals without $A \beta$ ( $A \beta$ - norms), which we validated in an independent cohort by comparing their predictive accuracy to published norms. For memory tests (AVLT Immediate and Delayed), the A $\beta$ - norms identified an additional group of individuals at risk of dementia. For non-memory tests, we found no difference. For all tests, the $\mathrm{A} \beta$ - norms were more sensitive in identifying individuals at risk of progression to dementia within 2 years.

Conclusion: Our results confirm the relationship between $A \beta$ and cognition in the preclinical stages of $\mathrm{AD}$. The use of $\mathrm{A} \beta$ - norms will improve prognostic sensitivity for memory tests when used in combination with published norms. This will be useful in clinical practice as well as for future research. 


\section{INTRODUCTION}

Neuropsychological examination is an essential element when diagnosing Alzheimer's disease $(\mathrm{AD})$. Normative data for neuropsychological tests enable interpretation of test performance and are typically based on cognitively normal individuals categorized by age, gender and years of education. However, pathological changes related to neurodegenerative diseases such as $\mathrm{AD}$, could already be present in aged cognitively normal individuals, ${ }^{1}$ and could lead to subtle changes in cognition. ${ }^{2-4}$ Still, norms for neuropsychological tests do not take AD biomarker status into account, which may lead to underdiagnosis of early stage $\mathrm{AD}$. The aim of the present study was to test whether norms based on individuals without AD pathology, would improve the sensitivity of cognitive neuropsychological tests to identify early AD.

The predominant hypothesis is that beta-amyloid $(\mathrm{A} \beta)$ is the first biomarker to become abnormal in $\mathrm{AD}$, followed by neuronal injury markers such as tau. ${ }^{5,6}$ To our knowledge, only one study has investigated the effect of these $\mathrm{AD}$ biomarkers on cognitive norms. ${ }^{7}$ In that study, it was found that individuals with normal levels of both $\mathrm{A} \beta$ and tau in cerebrospinal fluid (CSF) performed better on cognitive tests, but excluding these individuals with preclinical AD from normative datasets did not increase predictive accuracy of clinical decline in cognitively normal individuals. ${ }^{7}$ However, $A \beta$ and tau may independently impact cognitive performance in the preclinical stage in a test-specific manner. Also, it remains unknown whether biomarker negative norms would increase predictive accuracy of dementia in a memory clinic setting.

Hence in the present study, we first examined the independent effects of $A \beta$ and tau on five frequently used neuropsychological tests in a large sample of cognitively normal individuals. A $\beta$ was measured in CSF or on positron emission tomography (PET) and tau was measured in CSF. Subsequently, we calculated novel norms based on biomarker negative individuals for tests that were associated with $\mathrm{AD}$ biomarkers in the initial analyses. Lastly, we examined whether the use of these new norms could improve the identification of individuals at risk of progression to dementia among non-demented individuals from a memory clinic cohort.

\section{METHODS}

\section{Test dataset}

For the test dataset, 907 cognitively normal individuals were selected from eight cohorts of the European Medical Information Framework for Alzheimer's disease (EMIF-AD) 
project: Barcelona St. Pau, ${ }^{8,9}$ EDAR, ${ }^{10}$ Gipuzkoa Alzheimer Project (GAP), ${ }^{11}$ Gothenburg MCI study, ${ }^{12}$ IDIBAPS, ${ }^{13}$ IMAP+,${ }^{14}$ Leuven, ${ }^{15-17}$ EMIF preclinical-AD study, ${ }^{18}$ and from the American Alzheimer's Disease Neuroimaging Initiative (ADNI) study (Supplementary Text 1$).{ }^{19}$ Supplementary Table 1 shows an overview of the participating centers and the included number of subjects.

\section{Inclusion criteria}

Inclusion criteria were: 1) age above 40 years; 2) no cognitive impairment at baseline (Supplementary Table 1 provides an overview of definitions of $\mathrm{CN}$ by cohort); 3 ) availability of an A $\beta$ measurement in CSF or on amyloid positron emission tomography (PET); and 4) a baseline neuropsychological examination. From the EMIF preclinical-AD study we randomly selected one individual per monozygotic twin pair, to avoid duplication bias.

\section{Neuropsychological examination}

Neuropsychological examination was performed according to the routine protocol at each site, including the Mini-Mental State Examination $\left(\mathrm{MMSE}^{20}\right)$. For the current study, we selected the Auditory Verbal Learning Test (AVLT) immediate and delayed recall ${ }^{21}$ as a measure of immediate and delayed memory. The AVLT immediate recall score is the sum of 5 learning trials of a 15-word list, scores range from 0-75. In the AVLT delayed recall, the 15-word list has to be recalled after 20 minutes, so scores range from $0-15$. The Verbal Fluency Test (VFT) ${ }^{22}$ was used as a measure of verbal fluency, in which a person has to name as many animals as possible within one minute. The Trail Making Test (TMT) part A was used as measure of attention, in which a person has to connect numbers (1-25) in ascending order, as quickly as possible ${ }^{23}$. TMT part B was used as a measure of executive functioning in which a person should alternate between connecting numbers and letters in ascending order, as quickly as possible. For TMT part A and B time in seconds was used as an outcome.

\section{Biomarker analyses}

CSF biomarker assessments of A $\beta 1-42$, total tau ( $\mathrm{t}$-tau) and phosphorylated tau ( $\mathrm{p}$-tau) were performed according to the routine protocol in $n=569$ individuals. For the four cohorts that used a PET scan to measure amyloid ( $\mathrm{n}=338),\left[{ }^{18} \mathrm{~F}\right]$ flutemetamol or $\left[{ }^{18} \mathrm{~F}\right] \mathrm{AV} 45$ PET scans were visually rated locally or the standardized uptake value (SUV) was calculated. We used center-specific cut-offs to define abnormal biomarker values (Supplementary Table 1).

Amyloid positivity $(\mathrm{A} \beta+)$ was defined as an abnormal amyloid profile in CSF or on amyloid PET. Since the concordance between t-tau and p-tau was not similar across cohorts, tau positivity $(\mathrm{T}+)$ was defined as both abnormal t-tau and abnormal p-tau in CSF (Supplementary Table 1). 


\section{Validation dataset}

To test whether the new norms improved prediction of dementia in a clinical setting, we used an independent dataset from the Maastricht Alzheimer Centre Limburg (ACL) cohort ( $\mathrm{n}=1070$ ), including individuals from the ongoing, longitudinal study of patients referred to the Maastricht Memory Clinic. ${ }^{24}$ For the current study, we selected 1070 individuals based on the following inclusion criteria were: 1 ) age above 40 years; 2) no dementia diagnosis at baseline; 3) baseline data on at least one the following measures: AVLT immediate recall, AVLT delayed recall, VFT, TMT-A or TMT-B; and 4) minimally one clinical follow-up, at least 6 months after the baseline diagnosis.

In the validation dataset no biomarker information was used, only cognitive test performance and progression to dementia at follow-up. As the Maastricht ACL cohort is a memory clinic cohort, the validation dataset contained individuals with subjective or objective cognitive impairment at baseline.

\section{Clinical diagnosis}

We used a clinical diagnosis at follow-up as outcome measure that was made as part of standard clinical practice. Standard clinical practice includes: clinical interview, neuropsychological examination (raw scores and z-scores based on published norms) and magnetic resonance imaging (MRI). As CSF collection is not part of routine clinical practice this was usually not used when clinical diagnoses were made. Data on CSF biomarkers was available at time of diagnoses in a small subgroup $(n=104,10 \%)$. Z-scores based on biomarker negative normative data were not available at the time of clinical diagnosis. Diagnosis of dementia at follow-up was made according to Diagnostic and Statistical Manual of Mental Disorders, Fourth Edition. ${ }^{25}$ Clinical etiological diagnoses for subtypes of dementia were made according to standardized clinical criteria for AD-type dementia, ${ }^{26}$ vascular dementia, ${ }^{27}$ frontotemporal dementia, ${ }^{28}$ and Lewy body dementia. ${ }^{29}$ As etiological diagnoses were made without CSF biomarkers, we used a generic dementia diagnosis as main outcome measure.

\section{Cognitive data}

Cognitive data was available for the following neuropsychological tests: AVLT immediate and delayed recall, VFT, TMT-A and TMT-B. Standardized scores (z-scores) based on published norms were already available as they are used in standard current clinical practice. These published norms were adjusted for age, gender and years of education as described in previous publications: AVLT Immediate and Delayed recall, ${ }^{30} \mathrm{VFT},{ }^{31} \mathrm{TMT}$ part $\mathrm{A}$ and $\mathrm{B} .{ }^{32}$ In addition, we calculated novel $\mathrm{z}$-scores based on biomarker negative norms derived from the test dataset. 


\section{Statistical analyses}

\section{Analyses in test dataset}

As tau was only available in a subgroup of the test dataset, demographics were compared between $A \beta$ - and $A \beta+$ groups, using t-tests for continuous and Chi-square for categorical variables. General linear mixed models, with random intercept at study level, were used to assess the influence of biomarkers on neuropsychological test performance using the following method: for each cognitive outcome measure (AVLT Immediate, AVLT Delayed, VFT, TMT-A and TMT-B), we started with a standard model examining the influence of age, gender and years of education on test performance (model 1 ). Next, we added $\mathrm{A} \beta$ status (model $2=$ model $1+\mathrm{A} \beta$ ) and tau status (model $3=$ model $2+$ tau). In model 4 , we entered all variables from model 3 and tested all two-way interactions between variables using a forward selection method. All non-significant interactions were removed from the model, significant interactions are shown in Table 2. Based on the significant predictors for each neuropsychological test, we calculated adjusted standardized scores (z-scores) using a regression-based approach based on only the biomarker negative individuals (Table 3 ). Age and year of education were entered as continues variables, sex as a dichotomous variable (female $=0$, male $=1$ ). For the TMT-A and TMT-B the regression formulas were multiplied by -1 , such that a higher raw score indicated worse performance. We also performed sensitivity analyses regarding the method of defining A $\beta$ status (PET vs. CSF) by adding a dichotomous variable $(0=\mathrm{CSF}, 1=\mathrm{PET})$ to the statistical models.

\section{Analyses in validation dataset}

We classified the subjects in the validation dataset into three groups as illustrated in Figure 1: 1) individuals with a $\mathrm{z}$-score $\geq-1.5$ based on both published and $A \beta$ - norms: "Normal performance by both norms"; 2 ) individuals with a $\mathrm{z}$-score $\geq-1.5$ based on the published norms, but a $\mathrm{z}$-score $<-1.5$ based on the $\mathrm{A} \beta$ - norms: "Abnormal performance only by biomarker negative norms"; 3 ) individuals with a $z$-score $<-1.5$ based on both published and A $\beta$ - norms: "Abnormal by both norms". Cox proportional hazard models were used to calculate the relative risk of progression to dementia for the three groups, adjusted for age, gender and years of education, using all available follow-up measures. Besides long-term predicitive accuracy, as calculated with the cox proportional hazard models, also shortterm predictive accuracy is of added value in clinical practice. Therefore, we used a $2 \times 2$ contingency table to calculate odds ratios, sensitivity, specificity, positive predictive value (PPV) and negative predictive value (NPV) of progression to dementia within two years, given an abnormal performance according to the biomarker negative norms and the published norms. Statistical analyses were conducted using R Statistical Software (version 3.3.3) and SPSS version 24.0 (Chicago, IL, USA) with significance set at $\mathrm{p}<0.05$. 


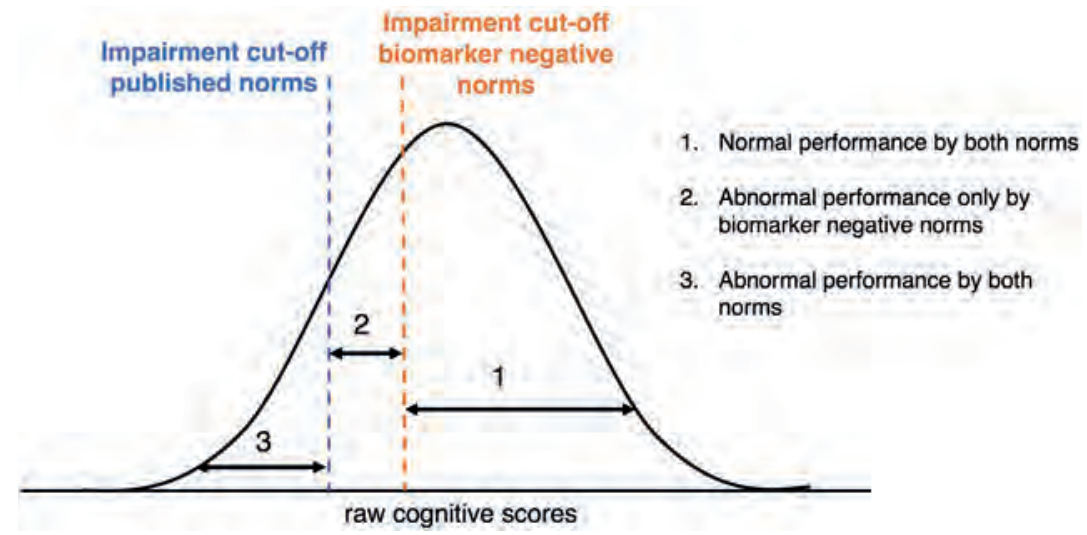

Figure 1. Schematic figure of classification according to published norms and $A \beta$ - norms

Graphical representation of distribution of raw cognitive scores and the different cut-points using published norms or $\mathrm{A} \beta$ - norms.

\section{RESULTS}

\section{Test dataset}

The test dataset consisted of 907 individuals with a mean age of 68.0 (SD 9.1) years and an average of 14.7 (SD 3.7) years of education. Four hundred and eighty-one (53\%) of these were female. In 334 (37\%) individuals, $A \beta$ was measured using PET. In the remaining 568 (63\%) individuals $\mathrm{A} \beta$ status was measured using CSF and these individuals also had data available on CSF tau status. Two hundred and twenty-nine (25\%) were A $\beta+$. Of the $n=569$ that had data on tau status available, $n=73(8 \%)$ were $T+$.

Table 1 shows the demographic and cognitive variables for $A \beta$ - and $A \beta+$ individuals. The $A \beta+$ group was significantly older $(\mathrm{p}<0.001)$, more likely to carry an APOE $\varepsilon 4$ allele $(\mathrm{p}<0.001)$ and had more often abnormal $\mathrm{t}$-tau and $\mathrm{p}$-tau levels $(\mathrm{p}<0.001)$, compared to the $A \beta$ - group. The groups did not differ in gender distribution ( $\mathrm{p}=0.490)$, years of education $(\mathrm{p}=0.907)$ or baseline MMSE score $(\mathrm{p}=0.801)$. Regarding cognition, the $\mathrm{A} \beta$ - group outperformed the $A \beta+$ group on the AVLT Immediate $(p=0.020)$, AVLT delayed $(\mathrm{p}<0.001)$, TMT-A $(\mathrm{p}=0.011)$ and TMT-B $(\mathrm{p}<0.001)$. There was no difference in performance on the VFT $(\mathrm{p}=0.178)$ (Table 1$)$. 
Table 1. Demographic and cognitive variables by $\mathrm{A} \beta$ status in test dataset

\begin{tabular}{|c|c|c|c|c|}
\hline Characteristics & $\begin{array}{l}\text { No. of subjects } \\
A \beta-/ A \beta+\end{array}$ & $A \beta-$ & $\mathrm{A} \beta+$ & p-value \\
\hline Age, years & $677 / 230$ & $66.7(14.7)$ & $71.8(8.6)$ & $<0.001$ \\
\hline Female, $\mathrm{n}$ & $677 / 230$ & $358(53 \%)$ & $128(56 \%)$ & 0.466 \\
\hline Education, years & $677 / 230$ & $14.7(3.7)$ & $14.7(3.6)$ & 0.907 \\
\hline APOE- $\varepsilon 4$ carrier, $n$ & $629 / 218$ & $174(28 \%)$ & $105(48 \%)$ & $<0.001$ \\
\hline MMSE, score & $677 / 230$ & $29.0(1.1)$ & $28.9(1.1)$ & 0.637 \\
\hline Abnormal CSF t-tau, $\mathrm{n}$ & $398 / 175$ & $42(11 \%)$ & $45(26 \%)$ & $<0.001$ \\
\hline Abnormal CSF p-tau, $n$ & $395 / 174$ & $100(25 \%)$ & $85(49 \%)$ & $<0.001$ \\
\hline AVLT Immediate, words recalled & $440 / 183$ & $45.5(10.1)$ & $43.5(9.4)$ & 0.020 \\
\hline AVLT Delayed, words recalled & $439 / 182$ & $8.8(3.4)$ & $7.7(3.6)$ & $<0.001$ \\
\hline VFT, words named & $526 / 190$ & $21.6(5.7)$ & $20.9(5.5)$ & 0.178 \\
\hline TMT-A, seconds & $674 / 230$ & $36.7(14.6)$ & $39.8(17.7)$ & 0.011 \\
\hline TMT-B, seconds & $670 / 228$ & $85.5(38.3)$ & $99.6(62.1)$ & $<0.001$ \\
\hline
\end{tabular}

Results are mean (SD) or frequency (\%). Abbreviations: $\mathrm{A} \beta=$ amyloid-beta, $\mathrm{APOE}=$ Apolipoprotein E, AVLT $=$ Auditory Verbal Learning Test, CSF = cerebrospinal fluid, MMSE = mini mental state examination, No. = number, $\mathrm{p}$-tau $=$ phosphorylated tau, TMT $=$ trail making test, $\mathrm{t}$-tau $=$ total tau, VFT $=$ verbal fluency test.

\section{Influence of $A \beta$ and tau on cognition}

Table 2 shows the outcomes of the linear mixed models which tested the influence of demographics and biomarkers on neuropsychological test performance. Age and education were associated with performance on all tests, and female gender was associated with a better performance on memory tests only (Table 2). A $\beta$ status was a significant predictor for the TMT part A and part B (all $\mathrm{p}<0.01$ ). When tau was added to the models (model 3 ), the associations between tau status and test performance did not reach significance for any of the neuropsychological tests. In model 4, with 2-way interactions, we found that for the AVLT Immediate, AVLT Delayed and TMT-B the effect of A $\beta$ depended on age, such that $A \beta$ status predicted performance only at higher ages (model 4; interaction $A \beta^{*}$ age: AVLT Immediate $p=0.048$; AVLT Delayed $p=0.025$; TMT-B $p=0.030$ ). The other 2 -way interactions were all found insignificant. Additionally, we tested the influence of tau pathology on cognition without adding $A \beta$ (model 3 without $A \beta$ ), and found no significant influence of tau on any of the cognitive tests (data not shown). The sensitivity analyses showed that the method of defining A $\beta$ status (CSF vs. PET) did not have an influence on the results. 


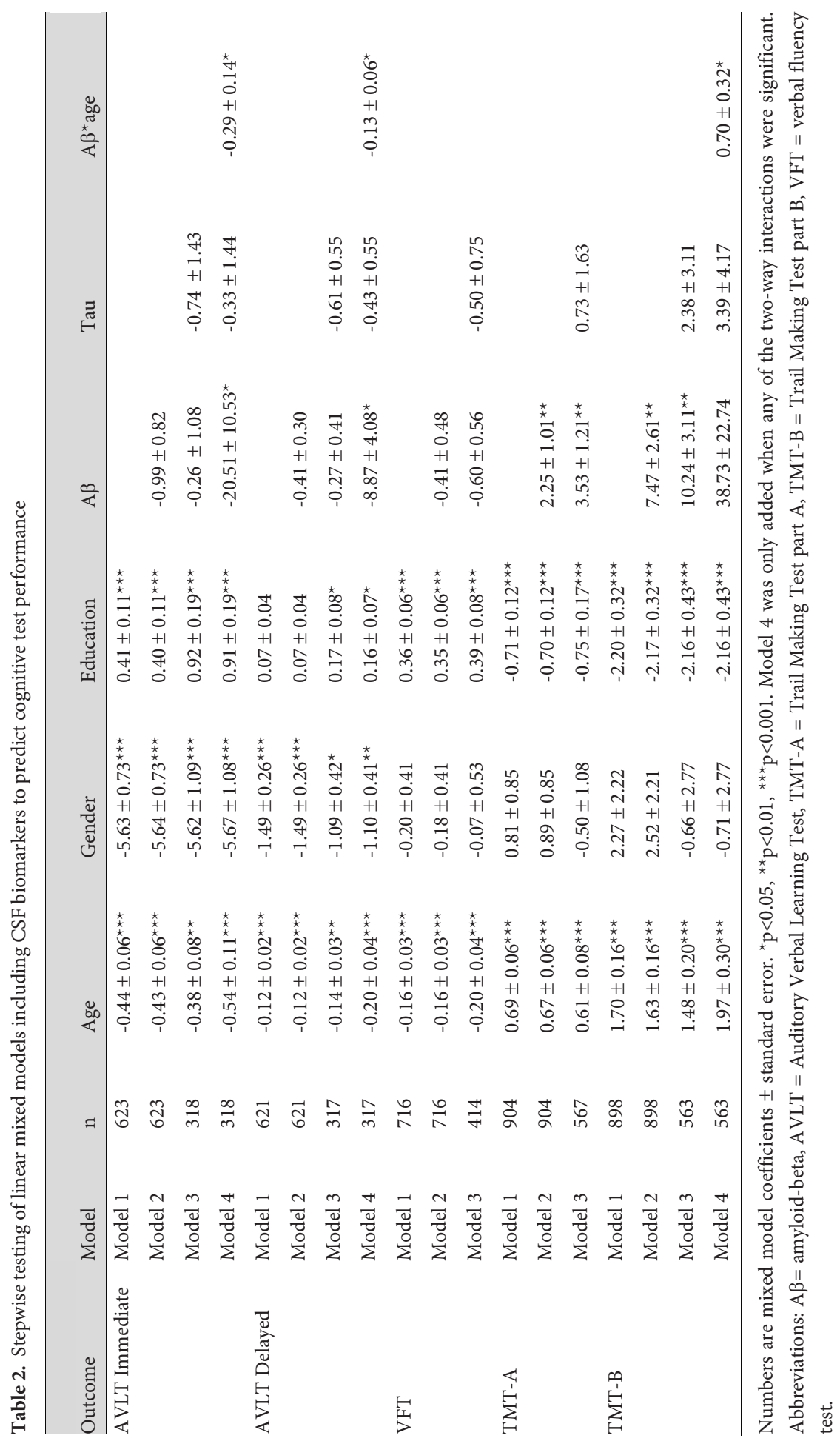




\section{A $\beta$ - norms}

Next, we calculated standardized normative scores (z-scores) for the AVLT Immediate and Delayed recall and the TMT part A and B based on A $\beta$ - individuals only. Table 3 shows the equations to calculate the $\mathrm{z}$-scores for the four cognitive measures, based on regression analyses.

Table 3: Z-scores equations based on $\mathrm{A} \beta$ - individuals

\begin{tabular}{|c|c|c|c|}
\hline $\begin{array}{l}\text { Neuropsychological } \\
\text { test }\end{array}$ & $\begin{array}{l}\text { Raw score } \\
\text { range }\end{array}$ & $\mathrm{n}$ & Z-score equations \\
\hline AVLT Immediate & $0-75$ & 440 & $\begin{array}{l}\text { Z-score }=\text { Raw score }-(68.396+-0.363 * A G E+ \\
-5.913 * \text { SEX }+0.358 * \text { EDUCATION }) / 3.048\end{array}$ \\
\hline AVLT Delayed & $0-15$ & 439 & $\begin{array}{l}\text { Z-score }=\text { Raw score }-\left(17.508+-0.117^{*} \text { AGE }+\right. \\
-1.586 * \text { SEX }+0.019 * \text { EDUCATION }) / 1.796\end{array}$ \\
\hline TMT-A & $15-100$ & 674 & $\begin{array}{l}\text { Z-score }=-1^{*}(\text { Raw score }-(20.020+0.433 * \text { AGE }+ \\
-0.839 * \text { EDUCATION }) / 3.587)\end{array}$ \\
\hline TMT-B & $20-200$ & 670 & $\begin{array}{l}\text { Z-score }=-1^{*}\left(\text { Raw score }-\left(37.609+1.251^{*} \text { AGE }+\right.\right. \\
\left.\left.-2.486^{*} \text { EDUCATION }\right) / 5.627\right)\end{array}$ \\
\hline
\end{tabular}

AVLT Immediate $=$ Auditory Verbal Learning Test sum of 5 learning trials; AVLT Delayed $=$ Auditory Verbal Learning Test delayed recall; TMT-A $=$ Trail Making Test part A; TMT-B $=$ Trail Making Test part B. Variables are coded as follows: Age (continuous variable); Sex: Female $=0$, Male $=1$; Education: years of education (continuous variable).

\section{Validation dataset}

To test the prognostic utility of the norms based on $A \beta$ - individuals, we used a validation cohort of 1070 memory clinic visitors who were free of dementia at baseline, but had subjective or objective cognitive deficits based on published normative data. Table 4 shows the characteristics of the validation cohort. The average age was 63.2 (SD 10.7) years and $41 \%$ were female (Table 4). Five hundred forty-seven (51\%) were cognitively impaired in at least one cognitive domain, according to published norms. The average follow-up was 5.2 (SD 3.8) years and at the last follow-up visit, 255 (24\%) had progressed to dementia (Table 4).

\section{Long-term prognostic utility of $A \beta$ - norms}

Table 5 shows the classifications (normal/abnormal) according to the $\mathrm{A} \beta$ - norms and the published norms for the AVLT Immediate, AVLT Delayed, TMT-A and TMT-B. Depending on the assessed test, $38-64 \%$ of the individuals had a normal performance according to both norms, $16-38 \%$ had an abnormal performance only according to the A $\beta$ norms and $17-31 \%$ had an abnormal performance according to both norms. Individuals who performed abnormal according to both norms on the AVLT Immediate, AVLT Delayed, TMT-A and TMT-B showed a faster progression rate to dementia, compared to 
individuals who performed normal according to the $\mathrm{A} \beta$ - norms $(\mathrm{p}<0.001)$, and those performing abnormal according to both norms $(\mathrm{p}<0.001)$ (Table 5; Figure 2). In addition, individuals who performed abnormal only according to the $A \beta$ - norms on the AVLT Immediate and AVLT delayed, progressed at a faster rate to dementia relative to individuals who performed normal according to both norms (AVLT Immediate $\mathrm{p}<0.001$; AVLT Delayed $\mathrm{p}=0.009$ ) (Table 5; Figure 2). We also calculated hazard ratios for an abnormal performance, relative to a normal performance, for published norms and A $\beta$ - norms (Supplemental Table 2).

When repeating the survival analyses with progression to clinical diagnosis of AD-type dementia as an outcome, results were similar (data not shown). Results were also comparable after exclusion of individuals for whom CSF biomarker data was available when the clinical diagnosis was made $(n=104)$. As we found in the analyses in the test cohort that age was related to the influence of $\mathrm{A} \beta$ on cognition, we also repeated the validation analyses in younger $(\leq 70)$ and older $(>70)$ individuals separately. Associations remained similar when stratifying by age, although associations were stronger in older individuals (data not shown).

Table 4: Characteristics of validation dataset

\begin{tabular}{ll}
\hline Characteristics & Total sample \\
\hline $\mathrm{n}$ & 1070 \\
Age, years & $63.2(10.7)$ \\
Female, $\mathrm{n}$ & $437(41 \%)$ \\
Education, years & $10.5(3.1)$ \\
Follow-up length, years & $5.2(3.8)$ \\
Progression to dementia, $\mathrm{n}$ & $251(24 \%)$ \\
- AD-type dementia, $\mathrm{n}$ & $170(16 \%)$ \\
- Vascular dementia, $\mathrm{n}$ & $21(2 \%)$ \\
- Frontotemporal dementia, $\mathrm{n}$ & $5(1 \%)$ \\
- Lewy Body or Parkinson dementia, $\mathrm{n}$ & $5(1 \%)$ \\
- Other dementia or unknown etiology, $\mathrm{n}$ & $50(5 \%)$ \\
Impairment in only one cognitive domain ${ }^{*}, \mathrm{n}$ & $328(31 \%)$ \\
Impairment in multiple cognitive domains ${ }^{*}, \mathrm{n}$ & $219(20 \%)$ \\
MMSE, score & $27.9(2.2)$ \\
AVLT Immediate, words recalled & $37.9(11.4)$ \\
AVLT Delayed, words recalled & $6.9(3.8)$ \\
VFT, words named & $19.9(6.3)$ \\
TMT-A, seconds & $50.0(19.0)$ \\
TMT-B, seconds & $91.1(44.8)$ \\
\hline
\end{tabular}

Results are mean (SD) or frequency (\%). ${ }^{\star}$ Based on $\mathrm{z}$-score $<-1.5$ derived from published normative data. Abbreviations: $\mathrm{AD}=$ Alzheimer's disease, $\mathrm{AVLT}=$ Auditory Verbal Learning Test, MMSE = Mini Mental State Examination, $\mathrm{TMT}=$ Trail Making Test, VFT $=$ Verbal Fluency Test. 


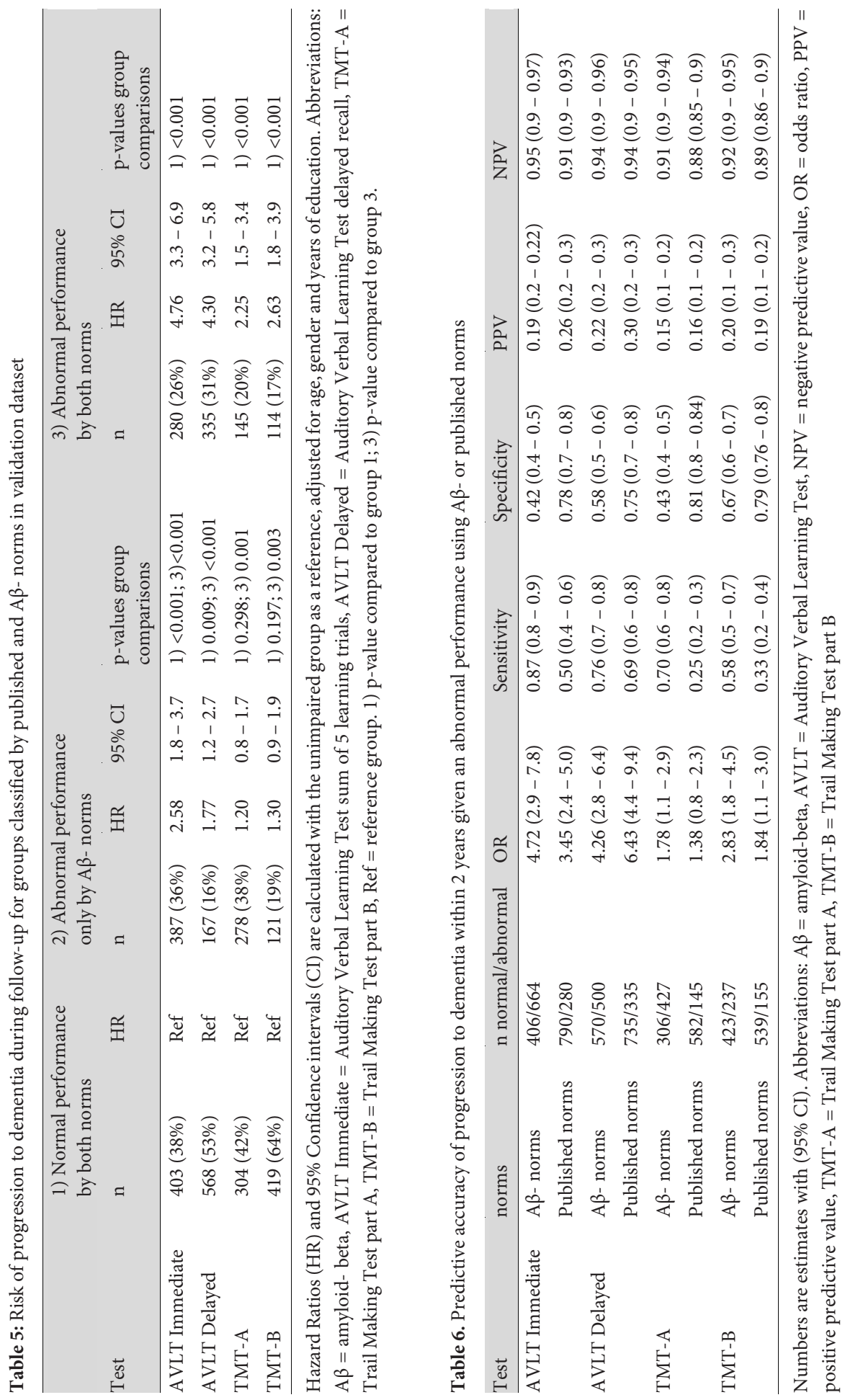



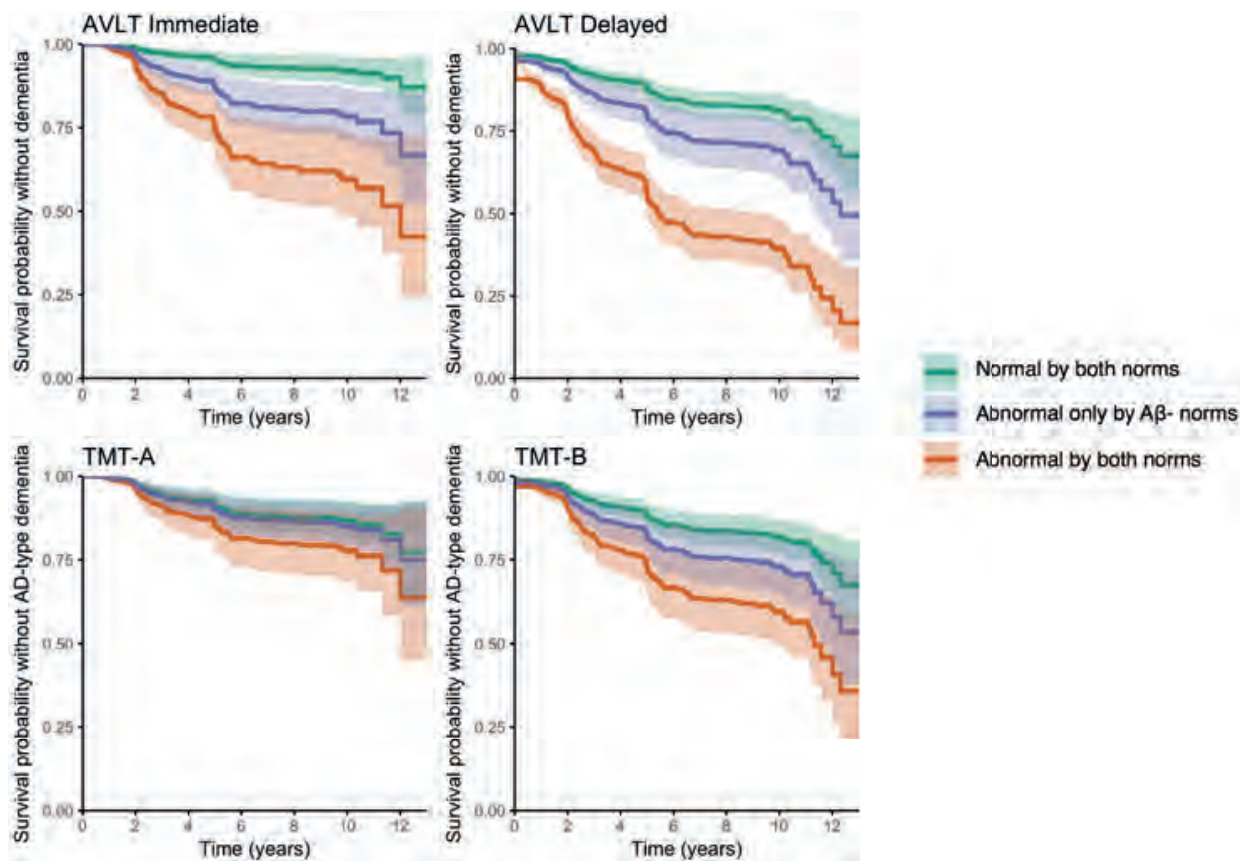

Abnormal by both norms

Figure 2. Progression to dementia for groups classified by current and $\mathrm{A} \beta$ - normative data in validation dataset

Survival probability without dementia for individuals who perform normal according to both norms (group 1), individuals who perform abnormal only according to $\mathrm{A} \beta$ - norms (group 2), individuals who perform abnormal according to both norms (group 3) on the AVLT Immediate (top left), AVLT Delayed (top right), TMT-A (bottom left) and TMT-B (bottom right). Survival curves are adjusted for age, gender and education.

\section{Short term predictive accuracy of $A \beta$ - and published norms}

Besides the long-term progression rates analysed with the survival analyses, we also investigated short-term progression rates which are of additive value in clinical practice. During two years of follow-up, 144 (14\%) of the individuals in the validation dataset progressed to dementia. Table 6 shows the short-term predictive accuracy measures of progression to dementia within two years since baseline visit, given an abnormal performance using the $\mathrm{A} \beta$ - norms or the published norms. When predicting risk of progression to dementia, odds were higher for A $\beta$ - norms for AVLT Immediate, TMT-A and TMT-B compared to published norms. For the AVLT Delayed, the odds of progression to dementia were higher for published norms compared to $A \beta$ - norms. The $A \beta$-norms had a higher sensitivity, but a lower specificity compared to the published norms for all cognitive tests (Table 6). We also calculated the predicative accuracy of being $A \beta+$ in the test dataset, results were comparable to the results with progression to dementia as an outcome (Supplemental Table 3). 


\section{DISCUSSION}

In a large cohort of cognitively normal individuals we tested the influence of $A \beta$ and tau biomarkers on cognitive test performance. We found that $A \beta$ influenced performance on all assessed cognitive tests, except for the VFT. The additional presence of tau did not further influence performance in any cognitive domain. For memory tests, we found that normative data based on only $\mathrm{A} \beta$ - individuals was more sensitive in indentifying individuals at risk of a faster progression to dementia. For non-memory tests, we found no difference between the $A \beta$ - norms and published norms.

\section{$A \beta$, tau and cognition}

Our study provides additional evidence that in cognitively normal individuals an abnormal $\mathrm{A} \beta$ biomarker negatively influences cognitive performance in the domains of memory, attention and executive functioning ${ }^{33-35}$. However, effects were small to moderate in particular in individuals below 70 years of age, which could explain why previous studies with smaller sample sizes and younger individuals reported no associations between $A \beta$ and cognition ${ }^{36}$. We found that tau did not influence cognitive test performance in addition to $A \beta$, nor in absence of $A \beta$. Moreover, we found that the influence of $A \beta$ on cognition was not influenced by tau status (i.e. A $\beta$-tau interaction). These findings are incongruent with previous findings in individuals with $\mathrm{MCI}$ as well as in cognitively normal individuals showing that tau and other neurodegenerative features, rather than $A \beta$, are associated with cognitive impairment and decline in various cognitive domains $s^{2,37-42}$. However, there are noteworthy differences between these previously mentioned studies and our study in determining tau status (CSF versus PET), cognitive outcome measure used (single test, composite score or computerized test) and design of the study (crosssectional cognitive performance versus longitudinal cognitive decline). Taken together, this may imply that the influence of abnormal CSF tau on baseline cognition performance on the tests that we investigated may be detectable only from the prodromal AD stage onwards (i.e. when cognitive impairments are already present). Future studies in different stages of the disease are needed to validate this notion.

\section{Prognostic utility of $A \beta$ - norms}

When comparing three groups classified by $\mathrm{A} \beta$ - norms and published norms in their progression rate to dementia, we showed that excluding $A \beta+$ individuals from normative data for memory tests increased the predictive accuracy for future progression to dementia. This seems to conflict with findings from a previous study ${ }^{7}$. However, in this previous study they investigated combined $A \beta$ - and tau- norms, instead of only $A \beta$-norms, and validated the norms in a preclinical cohort with progression to $\mathrm{CDR} \geq 0.5$ as an outcome, 
whereas we validated our norms in a large memory clinic cohort with progression to dementia as an outcome. For non-amnestic cognitive tests (i.e. TMT-A and TMT-B), we found that $A \beta$ pathology was associated with a worse performance, but the prognostic utility of the $A \beta$ - norms was comparable to published norms. This implies that although $\mathrm{A} \beta$ pathology influences performance in non-amnestic domains in the preclinical stage, only a severe non-amnestic cognitive impairment (i.e. impairment according to published norms) is predictive of progression to dementia.

\section{Sensitivity vs. specificity}

While we showed that individuals who performed normal by published but abnormal by A $\beta$-norms on memory tests (group 2) progressed faster to dementia than individuals who performed normal by $\mathrm{A} \beta$ - norms (group 1) during 12 years of follow-up, application of the $A \beta$ - norms only slightly improved short-term predictive accuracy (two years) compared to published norms (AVLT immediate, TMT A \& B). This could indicate that two years is too short as a follow-up or that the increase in sensitivity in detecting those at risk of progression to dementia by the $A \beta$ - norms, is accompanied by a decrease in specificity (i.e. individuals with abnormal scores according to $A \beta$ - norms who will not progress to dementia). It is however important to note that we only compared different normative data to each other in their predictive accuracy of dementia and did not compare the predictive value of the cognitive tests. Hence, it cannot be deduced from our results which neuropsychological test is the best predictor of $\mathrm{AD}$. Based on the comparisons between the published and $A \beta$ - norms, we recommend to use the $A \beta$ - norms in combination with published norms by creating three groups of memory classification instead of two, as illustrated in Figure 1, since this will improve prognostic accuracy for individual patients. For example, a 70 -year old male with 16 years of education who recalled between 29 and 38 words on the AVLT Immediate would be classified in group 2 (normal by published, abnormal by $\mathrm{A} \beta$ - norms), implying that he is at intermediate risk of progression to dementia. Should he have recalled less than 29 words he would have been classified in group 3 (abnormal by published norms) with the highest risk of progression, and in case he recalled more than 38 words he would have been classified in group 1 (normal by A $\beta$ norms) with the lowest risk of progression.

\section{Age}

Age was a consistent predictor of cognitive performance in all cognitive domains even after accounting for present $\mathrm{AD}$ pathology, confirming earlier findings ${ }^{7,43}$. Interestingly, older age augmented the effect of $A \beta$ on memory and executive functioning, which is in line with previous studies ${ }^{4,44}$. It is possible that at higher ages amyloid pathology is more 
extensive and thereby has a larger effect on cognition. Moreover, in older individuals amyloid pathology more often co-exists with other pathologies and together these pathologies may have an additive or synergistic effect on cognition, possibily through processes like inflammation ${ }^{45,46}$. Inflammation has been associated with many concomitant pathologies, but also with $\mathrm{A} \beta$ itself, and has shown to affects cognition, in particular at higher age $^{47,48}$.

\section{Strengths and limitations}

Strengths of the current study are the large sample sizes of both the test and validation datasets, assessment of commonly used neuropsychological tests and a long follow-up time in the validation cohort. Nevertheless, the results of the current study must be understood in the context of several limitations. In the test dataset, we included subjects from different centers, which might have led to variability in the data. In addition, it is likely that there was some form of selection bias in the test dataset, making it less comparable to other normative dataset which are usually a reflection of the general population. However, the test data set did not contain individuals with cognitive complaints or individuals from clinical settings. We used only five neuropsychological tests as outcome measure, using additional test might have yielded different results. Our test sample was relatively young, which makes the $A \beta$ - norms less reliable to assess cognition at higher ages. We used different methods for defining A $\beta$ pathology (CSF and PET) and we only had data on tau available in a subgroup (e.g. the group with CSF). However, sensitivity analyses showed no influence of the method assessing $A \beta$ and on the associations between $\mathrm{A} \beta+$ and cognition and these remained similar when testing them in subgroups with CSF (tau) data or PET data available (data not shown). Although the biomarker collection (CSF and PET) was performed according to local routine collection protocols, local cutoff points were used to define $A \beta$ status which is a frequently used method to pool biomarker data across studies ${ }^{49-51}$. Lastly, we only studied tau in CSF as a marker for neurodegeneration. Future studies should determine whether other neurodegenerative markers, like hippocampal atrophy on MRI or tau PET, might provide different results.

\section{Conclusion}

Using the $A \beta$ - norms has implications when assessing memory in clinical practice as well as in research settings. A disadvantage of using norms that do not take amyloid status into account (i.e. published norms) is that there is a substantial part of individuals that would be classified as having normal memory, while they may have underlying AD. Consequently it seems best to classify memory performance into three categories by combining $\mathrm{A} \beta$ - norms with published norms (Figure 1), rather than just classifying individuals as 
normal or abnormal by a single set of norms. The use of three groups for memory performance classification will improve diagnostic and prognostic accuracy for individual patients. Future studies should determine the diagnostic and prognostic values of using $A \beta$ norms for other cognitive tests. 


\section{REFERENCES}

1. Dubois B, Hampel H, Feldman HH, et al. Preclinical Alzheimer's disease: Definition, natural history, and diagnostic criteria. Alzheimer's \& dementia : the journal of the Alzheimer's Association. 2016;12(3):292-323.

2. Mormino EC, Betensky RA, Hedden T, et al. Synergistic effect of beta-amyloid and neurodegeneration on cognitive decline in clinically normal individuals. JAMA Neurol. 2014;71(11):1379-1385.

3. Vos SJ, Xiong C, Visser PJ, et al. Preclinical Alzheimer's disease and its outcome: a longitudinal cohort study. The Lancet Neurology. 2013;12(10):957-965.

4. Jansen WJ, Ossenkoppele R, Tijms BM, et al. Association of Cerebral Amyloid-beta Aggregation With Cognitive Functioning in Persons Without Dementia. JAMA Psychiatry. 2017.

5. Bateman RJ, Xiong C, Benzinger TL, et al. Clinical and biomarker changes in dominantly inherited Alzheimer's disease. The New England journal of medicine. 2012;367(9):795-804.

6. Jack CR, Knopman DS, Jagust WJ, et al. Tracking pathophysiological processes in Alzheimer's disease: an updated hypothetical model of dynamic biomarkers. The Lancet Neurology. 2013;12(2):207-216.

7. Hassenstab J, Chasse R, Grabow P, et al. Certified normal: Alzheimer's disease biomarkers and normative estimates of cognitive functioning. Neurobiology of aging. 2016;43:23-33.

8. Alcolea D, Carmona-Iragui M, Suarez-Calvet M, et al. Relationship between beta-Secretase, inflammation and core cerebrospinal fluid biomarkers for Alzheimer's disease. Journal of Alzheimer's disease : JAD. 2014;42(1):157-167.

9. Sala I, Illan-Gala I, Alcolea D, et al. Diagnostic and Prognostic Value of the Combination of Two Measures of Verbal Memory in Mild Cognitive Impairment due to Alzheimer's Disease. Journal of Alzheimer's disease : JAD. 2017;58(3):909-918.

10. Reijs BLR, Ramakers I, Elias-Sonnenschein L, et al. Relation of Odor Identification with Alzheimer's Disease Markers in Cerebrospinal Fluid and Cognition. Journal of Alzheimer's disease : JAD. 2017;60(3):1025-1034.

11. Estanga A, Ecay-Torres M, Ibanez A, et al. Beneficial effect of bilingualism on Alzheimer's disease CSF biomarkers and cognition. Neurobiology of aging. 2017;50:144-151.

12. Wallin A, Nordlund A, Jonsson M, et al. Alzheimer's disease--subcortical vascular disease spectrum in a hospital-based setting: Overview of results from the Gothenburg MCI and dementia studies. J Cereb Blood Flow Metab. 2016;36(1):95-113.

13. Fortea J, Sala-Llonch R, Bartres-Faz D, et al. Increased cortical thickness and caudate volume precede atrophy in PSEN1 mutation carriers. Journal of Alzheimer's disease : JAD. 2010;22(3):909-922.

14. La Joie R, Landeau B, Perrotin A, et al. Intrinsic connectivity identifies the hippocampus as a main crossroad between Alzheimer's and semantic dementia-targeted networks. Neuron. 2014;81(6):1417-1428.

15. Adamczuk K, De Weer AS, Nelissen N, et al. Functional Changes in the Language Network in Response to Increased Amyloid beta Deposition in Cognitively Intact Older Adults. Cereb Cortex. 2016;26(1):358-373.

16. Adamczuk K, Schaeverbeke J, Nelissen N, et al. Amyloid imaging in cognitively normal older adults: comparison between (18)F-flutemetamol and (11)C-Pittsburgh compound B. European journal of nuclear medicine and molecular imaging. 2016;43(1):142-151.

17. Adamczuk K, Schaeverbeke J, Vanderstichele HM, et al. Diagnostic value of cerebrospinal fluid Abeta ratios in preclinical Alzheimer's disease. Alzheimer's research \& therapy. 2015;7(1):75.

18. Demuru M, Gouw AA, Hillebrand A, et al. Functional and effective whole brain connectivity using magnetoencephalography to identify monozygotic twin pairs. Scientific reports. 2017;7(1):9685.

19. Mueller SG, Weiner MW, Thal LJ, et al. Ways toward an early diagnosis in Alzheimer's disease: the Alzheimer's Disease Neuroimaging Initiative (ADNI). Alzheimer's \& dementia : the journal of the Alzheimer's Association. 2005;1(1):55-66.

20. Folstein MF, Folstein SE, McHugh PR. "Mini-mental state". A practical method for grading the cognitive state of patients for the clinician. Journal of psychiatric research. 1975;12(3):189-198. 
21. Rey A. L'examen clinique en psychologie [the psychological examination]. Paris: Presses Universitaires de Frances; 1958.

22. Lezak MD, Howieson DB, Loring DW. Neuropsychological Assessment. Oxford, UK: Oxford University Press 2004.

23. Reitan R. Trail-Making Test. Arizona: Reitan Neuropsychology Laboratory 1979.

24. Visser PJ, Kester A, Jolles J, Verhey F. Ten-year risk of dementia in subjects with mild cognitive impairment. Neurology. 2006;67(7):1201-1207.

25. APA. Diagnostic and Statistical Manual of Mental Disorders: DSM-IV. Washington, DC: American Psychiatric Association; 1994.

26. McKhann G, Drachman D, Folstein M, Katzman R, Price D, Stadlan EM. Clinical diagnosis of Alzheimer's disease: report of the NINCDS-ADRDA Work Group under the auspices of Department of Health and Human Services Task Force on Alzheimer's Disease. Neurology. 1984;34(7):939-944.

27. Roman GC, Tatemichi TK, Erkinjuntti T, et al. Vascular dementia: diagnostic criteria for research studies. Report of the NINDS-AIREN International Workshop. Neurology. 1993;43(2):250-260.

28. Neary D, Snowden JS, Gustafson L, et al. Frontotemporal lobar degeneration: a consensus on clinical diagnostic criteria. Neurology. 1998;51(6):1546-1554.

29. McKeith IG, Galasko D, Kosaka K, et al. Consensus guidelines for the clinical and pathologic diagnosis of dementia with Lewy bodies (DLB): report of the consortium on DLB international workshop. Neurology. 1996;47(5):1113-1124.

30. Van der Elst W, van Boxtel MP, van Breukelen GJ, Jolles J. Rey's verbal learning test: normative data for 1855 healthy participants aged 24-81 years and the influence of age, sex, education, and mode of presentation. Journal of the International Neuropsychological Society: JINS. 2005;11(3):290-302.

31. Van der Elst W, Van Boxtel MP, Van Breukelen GJ, Jolles J. Normative data for the Animal, Profession and Letter M Naming verbal fluency tests for Dutch speaking participants and the effects of age, education, and sex. Journal of the International Neuropsychological Society: JINS. 2006;12(1):80-89.

32. Norms for Stroop Color Word test, Trail making test, and Story recall of Rivermead Behavioural Memory Test. 2003.

33. Baker JE, Lim YY, Pietrzak RH, et al. Cognitive impairment and decline in cognitively normal older adults with high amyloid- $\beta$ : A meta-analysis. Alzheimer's \& Dementia: Diagnosis, Assessment \& Disease Monitoring. 2017.

34. Mielke MM, Machulda MM, Hagen CE, et al. Influence of amyloid and APOE on cognitive performance in a late middle-aged cohort. Alzheimer's \& dementia : the journal of the Alzheimer's Association. 2016;12(3):281-291.

35. Petersen RC, Wiste HJ, Weigand SD, et al. Association of elevated amyloid levels with cognition and biomarkers in cognitively normal people from the community. JAMA neurology. 2016;73(1).

36. Hedden T, Oh H, Younger AP, Patel TA. Meta-analysis of amyloid-cognition relations in cognitively normal older adults. Neurology. 2013;80(14):1341-1348.

37. Nelson PT, Alafuzoff I, Bigio EH, et al. Correlation of Alzheimer disease neuropathologic changes with cognitive status: a review of the literature. Journal of neuropathology and experimental neurology. 2012;71(5):362-381.

38. Degerman Gunnarsson M, Ingelsson M, Blennow K, Basun H, Lannfelt L, Kilander L. High tau levels in cerebrospinal fluid predict nursing home placement and rapid progression in Alzheimer's disease. Alzheimer's research \& therapy. 2016;8(1):22.

39. Cerami C, Dodich A, Iannaccone S, et al. A biomarker study in long-lasting amnestic mild cognitive impairment. Alzheimer's research \& therapy. 2018;10(1):42. 
40. Brier MR, Gordon B, Friedrichsen K, et al. Tau and Abeta imaging, CSF measures, and cognition in Alzheimer's disease. Sci Transl Med. 2016;8(338):338ra366.

41. Dumurgier J, Hanseeuw BJ, Hatling FB, et al. Alzheimer's Disease Biomarkers and Future Decline in Cognitive Normal Older Adults. Journal of Alzheimer's disease : JAD. 2017;60(4):1451-1459.

42. Pettigrew C, Soldan A, Moghekar A, et al. Relationship between cerebrospinal fluid biomarkers of Alzheimer's disease and cognition in cognitively normal older adults. Neuropsychologia. 2015;78:63-72.

43. Oh H, Madison C, Haight TJ, Markley C, Jagust WJ. Effects of age and beta-amyloid on cognitive changes in normal elderly people. Neurobiology of aging. 2012;33(12):2746-2755.

44. Lim YY, Laws SM, Villemagne VL, et al. Abeta-related memory decline in APOE epsilon4 noncarriers: Implications for Alzheimer disease. Neurology. 2016;86(17):1635-1642.

45. Vemuri P, Knopman DS. The role of cerebrovascular disease when there is concomitant Alzheimer disease. Biochimica et biophysica acta. 2016;1862(5):952-956.

46. Franceschi C, Campisi J. Chronic inflammation (inflammaging) and its potential contribution to ageassociated diseases. The journals of gerontology Series A, Biological sciences and medical sciences. 2014;69 Suppl 1:S4-9.

47. Simen AA, Bordner KA, Martin MP, Moy LA, Barry LC. Cognitive dysfunction with aging and the role of inflammation. Ther Adv Chronic Dis. 2011;2(3):175-195.

48. Giunta B, Fernandez F, Nikolic WV, et al. Inflammaging as a prodrome to Alzheimer's disease. J Neuroinflammation. 2008;5:51.

49. Jansen WJ, Ossenkoppele R, Knol DL, et al. Prevalence of cerebral amyloid pathology in persons without dementia: a meta-analysis. Jama. 2015;313(19):1924-1938.

50. Bos I, Vos SJ, Frölich L, et al. The frequency and influence of dementia risk factors in prodromal Alzheimer's disease. Neurobiology of aging. 2017.

51. Vos SJ, Verhey F, Frolich L, et al. Prevalence and prognosis of Alzheimer's disease at the mild cognitive impairment stage. Brain: a journal of neurology. 2015;138(Pt 5):1327-1338. 
Amyloid- $\beta$, tau and cognition in cognitively normal individuals

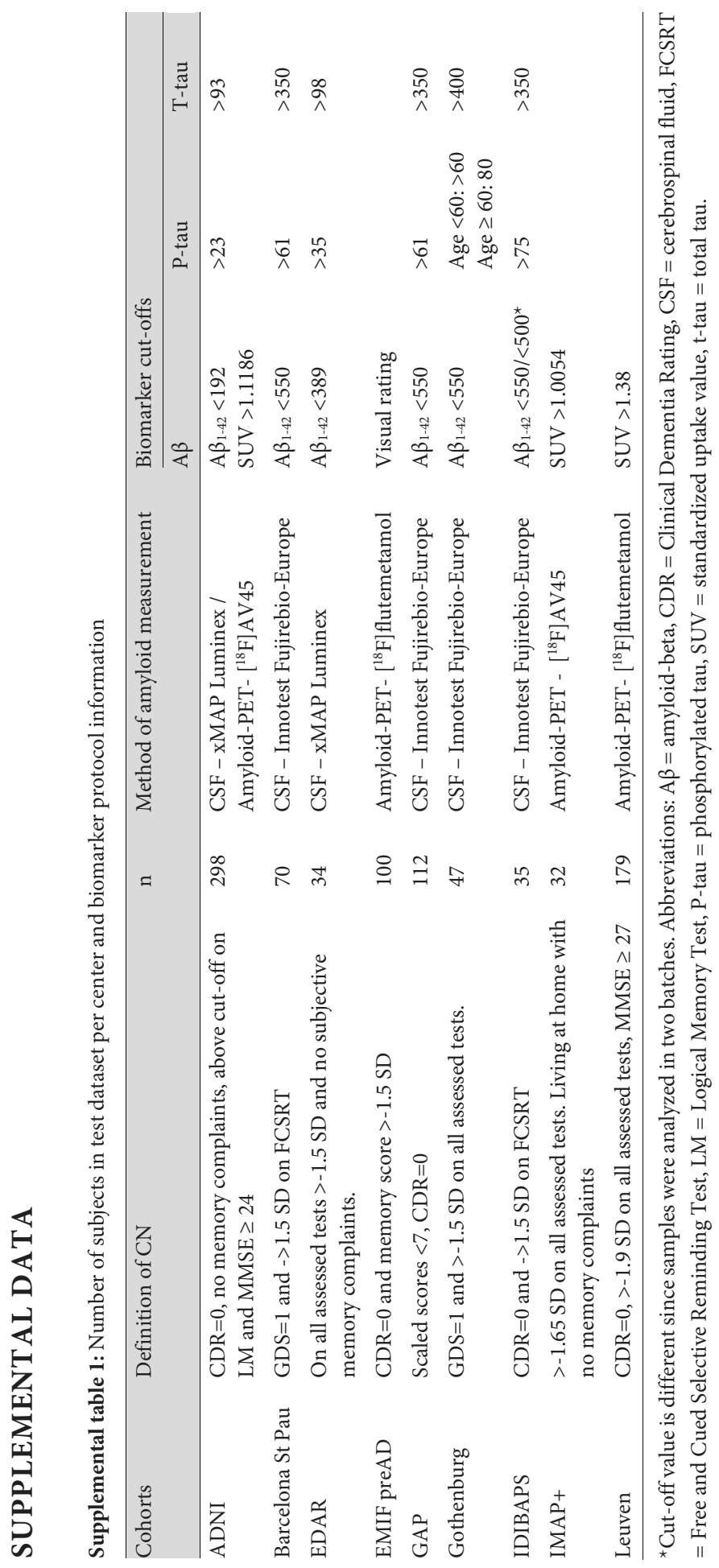


Supplemental Table 2: Risk of progression to dementia for abnormal performance based on published and A $\beta$ norms

\begin{tabular}{|c|c|c|c|c|c|c|}
\hline \multirow[b]{2}{*}{ Test } & \multicolumn{3}{|c|}{$\begin{array}{l}\text { Abnormal performance by } \\
\text { Published norms }\end{array}$} & \multicolumn{3}{|c|}{$\begin{array}{l}\text { Abnormal performance by } \\
A \beta \text { - norms }\end{array}$} \\
\hline & HR & $95 \% \mathrm{CI}$ & $\mathrm{p}$-value & HR & $95 \%$ CI & $\mathrm{p}$-value \\
\hline AVLT Immediate & 2.66 & $2.1-3.4$ & $<0.001$ & 3.38 & $2.4-4.7$ & $<0.001$ \\
\hline AVLT Delayed & 3.73 & $2.9-4.8$ & $<0.001$ & 3.43 & $2.6-4.6$ & $<0.001$ \\
\hline TMT-A & 2.03 & $1.4-2.9$ & $<0.001$ & 1.43 & $1.0-2.0$ & 0.028 \\
\hline TMT-B & 1.68 & $1.2-2.3$ & 0.002 & 1.65 & $1.2-2.3$ & 0.003 \\
\hline
\end{tabular}

$\mathrm{HR}$ are calculated with normal performing group, according to used norms, as a reference. Abbreviations: $\mathrm{A} \beta$ $=$ amyloid-beta, AVLT $=$ Auditory Verbal Learning Test, $\mathrm{CI}=$ confidence interval, $\mathrm{HR}=$ hazard ratio, $\mathrm{TMT}=$ Trail Making Test.

Supplemental Table 3: Predictive accuracy of being A $\beta+$ given an abnormal performance using $A \beta$ - or published norms

\begin{tabular}{lllllll}
\hline Test & Norms & OR & Sens & Spec & PPV & NPV \\
\hline AVLT & A $\beta$ - norms & $1.30(0.9-1.9)$ & $0.38(0.3-0.5)$ & $0.68(0.6-0.7)$ & $0.33(0.3-0.4)$ & $0.73(0.7-0.8)$ \\
Immediate & Pub. norms & $0.9(0.4-2.3)$ & $0.04(.01-.07)$ & $0.96(0.9-0.98)$ & $0.28(0.1-0.5)$ & $0.71(0.7-0.74)$ \\
AVLT & A $\beta$ - norms & $1.45(0.96-2.2)$ & $0.81(0.8-0.84)$ & $0.26(0.2-0.3)$ & $0.36(0.3-0.4)$ & $0.72(0.7-0.8)$ \\
Delayed & Pub. norms & $1.96(1.2-3.1)$ & $0.21(0.1-0.3)$ & $0.88(0.85-0.9)$ & $0.42(0.3-0.5)$ & $0.73(0.7-0.8)$ \\
TMT-A & A $\beta$ - norms & $1.07(0.8-1.5)$ & $0.28(0.2-0.3)$ & $0.73(0.7-0.8)$ & $0.26(0.2-0.3)$ & $0.75(0.7-0.8)$ \\
& Pub. norms & $0.98(0.5-2.1)$ & $0.04(.01-0.1)$ & $0.96(0.9-0.97)$ & $0.25(0.1-0.4)$ & $0.75(0.7-0.8)$ \\
TMT-B & A $\beta$ - norms & $1.05(0.8-1.4)$ & $0.32(0.3-0.4)$ & $0.69(0.6-0.7)$ & $0.26(0.2-0.3)$ & $0.75(0.7-0.8)$ \\
& Pub. norms & $0.99(0.6-1.6)$ & $0.10(0.1-0.14)$ & $0.90(0.9-0.92)$ & $0.25(0.2-0.3)$ & $0.75(0.7-0.8)$ \\
\hline
\end{tabular}

Numbers are estimates with $(95 \% \mathrm{CI})$. Abbreviations: $\mathrm{A} \beta=$ amyloid-beta, AVLT $=$ Auditory Verbal Learning Test, $\mathrm{NPV}=$ negative predictive value, $\mathrm{OR}=$ odds ratio, $\mathrm{PPV}=$ positive predictive value, $\mathrm{Pub} .=$ Published, TMT-A $=$ Trail Making Test part A, TMT-B = Trail Making Test part B 


\section{Supplemental Text 1: Methods ADNI Study}

Data used in the preparation of this article were partially obtained from the Alzheimer's Disease Neuroimaging Initiative (ADNI) database (adni.loni.usc.edu). The ADNI was launched in 2003 by the National Institute on Aging (NIA), the National Institute of Biomedical Imaging and Bioengineering (NIBIB), the Food and Drug Administration (FDA), private pharmaceutical companies and non-profit organizations, as a $\$ 60$ million, 5-year public-private partnership. The primary goal of ADNI has been to test whether serial magnetic resonance imaging (MRI), positron emission tomography (PET), other biological markers, and clinical and neuropsychological assessment can be combined to measure the progression of mild cognitive impairment (MCI) and early Alzheimer's disease(AD). Determination of sensitive and specific markers of very early $\mathrm{AD}$ progression is intended to aid researchers and clinicians to develop new treatments and monitor their effectiveness, as well as lessen the time and cost of clinical trials. The Principal Investigator of this initiative is Michael W. Weiner, MD, VA Medical Center and University of California-San Francisco. ADNI is the result of efforts of many co-investigators from a broad range of academic institutions and private corporations, and subjects have been recruited from over 50 sites across the U.S. and Canada. The initial goal of ADNI was to recruit 800 subjects but ADNI has been followed by ADNI-GO and ADNI-2. To date these three protocols have recruited over 1500 adults, ages 55 to 90 , to participate in the research, consisting of cognitively normal older individuals, people with early or late MCI, and people with early AD. The follow up duration of each group is specified in the protocols for ADNI-1, ADNI-2 and ADNI-GO. Subjects originally recruited for ADNI-1 and ADNI-GO had the option to be followed in ADNI-2. For up-to-date information, see www.adni-info.org 


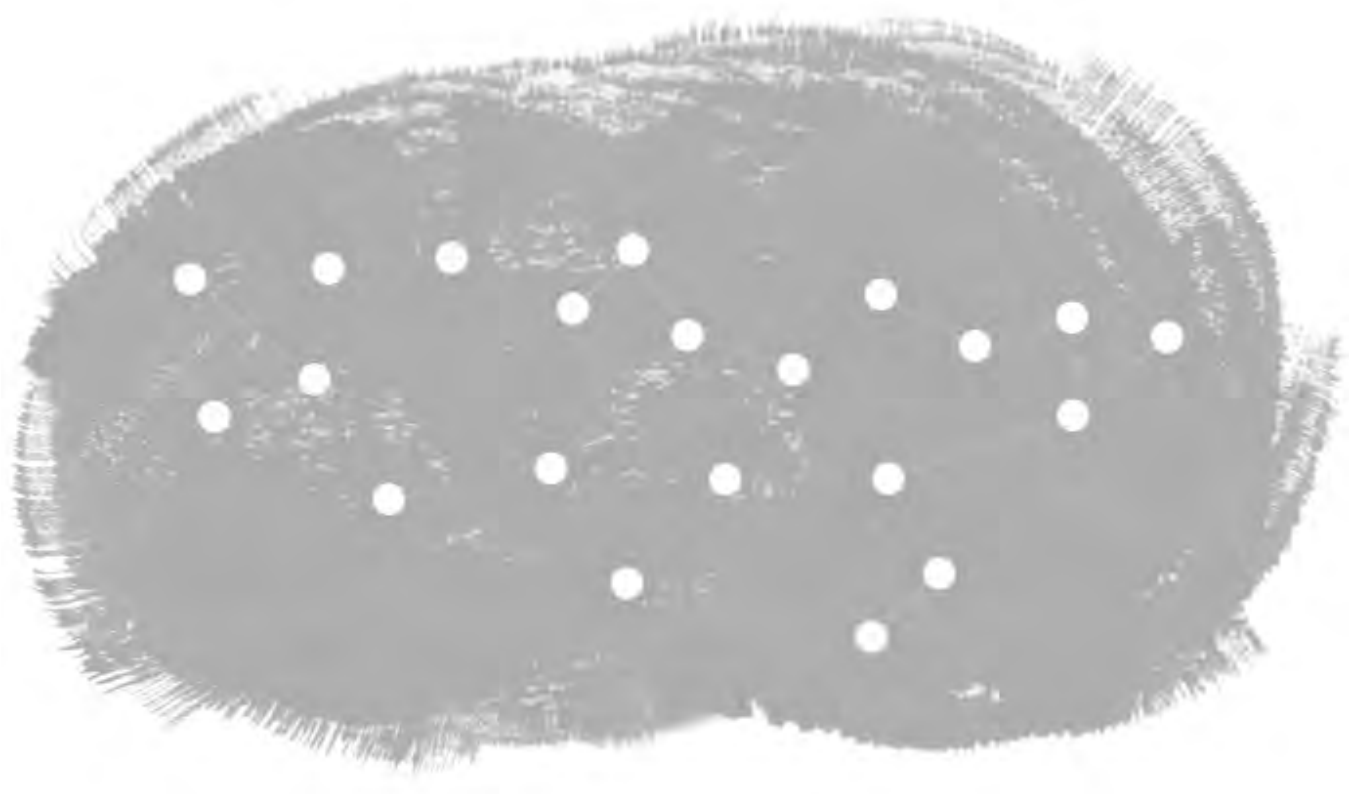




\begin{abstract}
Background: Amyloid-beta $(A \beta)$ deposition is an early event in Alzheimer's disease (AD) and apolipoprotein $\mathrm{E}(A P O E) \varepsilon 4$ carriership is the strongest genetic $\mathrm{AD}$ risk factor. We investigated the relationship between $\mathrm{A} \beta$ status, $A P O E \varepsilon 4$ carriership and cognition, with cerebrospinal fluid (CSF) markers of synaptic dysfunction (Neurogranin, Ng), axonal degeneration (Neurofilament-light, NFL) and astroglial activation (YKL-40), across the clinical AD spectrum.
\end{abstract}

Methods: We included 770 individuals (140 cognitively normal (CN), 450 mild cognitive impairment (MCI), 180 with AD-type dementia) from the EMIF-AD Multimodal Biomarker Discovery study for whom CSF analyses were conducted centrally. The $A \beta_{42 / 40}$ ratio was used to determine $A \beta$ status $(\mathrm{A} \beta-/+)$. We compared demographics and biomarker values by $A \beta$ status and diagnosis. Correlations between CSF biomarkers were assessed by Spearman's correlations. LMM was used to compare NFL, Ng and YKL-40 concentrations by APOE $\varepsilon 4$ status, and to test the influence of these markers on cognitive decline, all stratified by $\mathrm{A} \beta$ status.

Results: Compared to $\mathrm{A} \beta$ - individuals, $\mathrm{Ng}$ and t-tau levels were elevated in $\mathrm{A} \beta+$ individuals across the AD spectrum, while NFL and YKL-40 levels were elevated in $A \beta+$ in the pre-dementia stages but in the dementia stage they were elevated regardless of $A \beta$ status. $\mathrm{A} \beta$ - APOE \&4 carriers with MCI or AD-type dementia, had lower levels of NFL and Ng than non-carriers. High NFL was associated with lower baseline MMSE scores and a faster rate of decline in the total group, regardless of $\mathrm{A} \beta$ status. High $\mathrm{Ng}$ was also associated with a faster rate of cognitive decline, regardless of $A \beta$ status, only in the dementia stage. High YKL-40 was associated with lower baseline MMSE scores and a faster rate of decline only in $\mathrm{A} \beta$ - individuals with MCI or AD-type dementia.

Conclusion: Axonal degeneration, synaptic dysfunction and neuroinflammation are involved in $\mathrm{AD}$ pathophysiology already in the preclinical stage. All three mechanisms are also associated with cognitive decline, but this is dependent on $\mathrm{A} \beta$ status and disease stage. 


\section{INTRODUCTION}

Biomarkers have become increasingly important for the early diagnosis of Alzheimer's disease $(\mathrm{AD}),{ }^{1,2}$ and are contributing to an improved understanding of the temporal pattern of $\mathrm{AD}$ pathophysiology. It has been shown that amyloid-beta $(\mathrm{A} \beta)$ deposition is one of the earliest detectable events in $\mathrm{AD}$ pathogenesis, ${ }^{3,4}$ and that genetic risk for $\mathrm{AD}$ can be assessed by determining apolipoprotein $\mathrm{E}(A P O E) \varepsilon 4$ genotype, which is the strongest known genetic risk factor for AD. ${ }^{5}$ However, other pathophysiological mechanisms underlying $\mathrm{AD}$ and the inter-individual variation in cognitive trajectories, are less well understood. By relating A $\beta$, APOE genotype and cognition to cerebrospinal fluid (CSF) biomarkers for $\mathrm{AD}$-related processes including axonal degeneration, synaptic dysfunction and astroglial activation in individuals across the clinical AD spectrum, we will likely learn more about the temporal ordering of these pathological mechanisms. This may translate into improved diagnostic and prognostic algorithms, which, in turn, should help to develop and evaluate more targeted disease-modifying treatments.

Besides $A \beta$, a number of proteins in CSF have been found to be associated with $A D$ and are therefore considered potential biomarkers for the disease. High concentrations of neurofilament-light (NFL) in CSF have been associated with axonal degeneration to, predominantly, subcortical brain areas ${ }^{6,7}$ and YKL-40 (also known as chitinase 3-like protein 1) concentrations in CSF were found to reflect astrocytic activation, an inflammatory response to neurodegenerative processes. ${ }^{8}$ Although both axonal degeneration and astrocytic activation have also been related to other neurodegenerative diseases, ${ }^{8,9}$ it is important to increase understanding of their role in the development and course of AD. Neurogranin $(\mathrm{Ng})$ has been identified as a candidate AD marker reflecting synaptic degeneration and cognitive decline in the early stages of AD in CSF. ${ }^{10,11}$ While NFL, YKL40 and $\mathrm{Ng}$ have evolved over the last years as promising $\mathrm{AD}$ biomarkers and have been strongly associated with neuronal injury markers, ${ }^{11-13}$ data regarding their relation to $A \beta$, $A P O E$ and cognition have been inconsistent or inconclusive. ${ }^{10,12,14-16}$

Hence, to unravel how NFL, Ng and YKL-40 relate to AD pathology, genetic risk and disease severity, we aim to investigate their relationships with $\mathrm{A} \beta, A P O E \varepsilon 4$ carriership and cognition, in a large cohort consisting of individuals across the $\mathrm{AD}$ spectrum. To compare the relations regarding NFL, Ng and YKL-40 to those of an established neurodegenerative $\mathrm{AD}$ marker, we also examine the associations of total tau ( $\mathrm{T}$-tau) with $\mathrm{A} \beta$, $A P O E$ genotype and cognition. 


\section{METHODS}

\section{Subjects}

Seven hundred seventy individuals were selected from the EMIF-AD Multimodal Biomarker Discovery (EMIF-AD MBD) study; a cross-cohort study consisting of collated data and samples from 11 European cohorts aiming to discover novel diagnostic and prognostic markers for AD using multiple biomarker modalities. ${ }^{17}$ The EMIF-AD MBD includes a total of 1221 individuals across the cognitive spectrum: normal cognition (NC), Mild Cognitive Impairment (MCI) and AD-type dementia. Individuals were selected from prospective cohort studies based on the availability of plasma, DNA and CSF samples and MRI scans. Exclusion criteria for the EMIF-AD MBD study were the presence of neurological, psychiatric or somatic disorders that could cause cognitive impairment. ${ }^{17}$

For the current study, we selected all participants from whom CSF samples were available for central analyses $(n=770)$. Participants were included from three multicenter studies: DESCRIPA ( $\mathrm{n}=29),{ }^{18} \operatorname{EDAR}(\mathrm{n}=197){ }^{19}$ and PharmaCog $(\mathrm{n}=146),{ }^{20}$ and four single center studies: Amsterdam $(n=170),{ }^{21}$ Antwerp $(n=148),{ }^{22}$ San Sebastian GAP $(n=40)^{23}$ and Lausanne $(n=40){ }^{24}$

\section{Clinical diagnosis and assessment}

Normal cognition (NC) was defined as normal performance on neuropsychological assessment (within 1.5 SD of the average for age, gender and education). MCI was defined as having cognitive complaints and performance below $1.5 \mathrm{SD}$ of the average on at least one neuropsychological test. ${ }^{25} \mathrm{AD}$-type dementia was defined based on a clinical diagnosis, using the National Institute of Neurological and Communicative Disorders and Stroke - Alzheimer's Disease and Related Disorders Association (NINCDS-ADRDA) criteria. $^{26}$

The clinical assessment is described in detail in a previous publication. ${ }^{17}$ In short, clinical data were collected using local routine protocol at each site and thereafter harmonized and stored onto the EMIF-AD online data platform for pooled analyses. We used the Mini Mental State Examination (MMSE) ${ }^{27}$ as our main cognitive outcome measure, which was available in $99 \%$ of the subjects at baseline $(n=768)$ and in $68 \%(n=525)$ at follow-up (mean 2.3 years). In general, baseline clinical assessment and CSF collection were conducted within a one year window. For a subgroup, the length of this time window was unknown $(n=21)$ or longer than one year $(n=2)$.

\section{CSF analyses}

Central CSF analyses were conducted at Gothenburg University, Sweden. NFL concentration was measured using a commercial ELISA (NF-light ${ }^{\circledR}$ ELISA, Uman Diagnostics, 
Umeå, Sweden ${ }^{6}$. Ng was measured using an in-house immunoassay for $\mathrm{Ng} .{ }^{10} \mathrm{YKL}-40$ was determined by a human chitinase-3 quantikine ELISA kit (R\&D systems, Inc, Minneapolis, $M N^{28}$ ). $A \beta_{38}, A \beta_{40}$, and $A \beta_{42}$ were measured using the V-PLEX Plus $A \beta$ Peptide Panel 1 (6E10) Kit from Meso Scale Discovery (MSD, Rockville, MD). All analyses were performed according to the manufacturer's instructions by board-certified laboratory technicians who were blinded to clinical information. All measurement were performed on one occasion using one batch of reagents, except for $n=8$ samples from the EDAR cohort that were analysed beforehand in the same laboratory, but in a different batch. For phosphorylated tau ( $\mathrm{p}$-tau) and total tau ( $\mathrm{t}$-tau), we used available measures from the local cohorts ( $\mathrm{p}$-tau $\mathrm{n}=630 ; \mathrm{t}$-tau $\mathrm{n}=621$ ) derived in clinical laboratory practice using INNOTEST ELISAs (Fujirebio, Ghent, Belgium).

\section{Genetic analyses}

For the entire EMIF-AD BMD cohort $A P O E$ genotyping data from the local genetic analyses was available for $\mathrm{n}=1121$ (91\%) individuals. For central genetic analyses, 742 DNA and 187 whole blood samples were transferred to Lübeck University, Germany. From the blood samples, DNA was extracted using QIAamp ${ }^{\circledR}$ DNA Blood Mini Kit (QIAGEN GmbH, Hilden, Germany) resulting in 929 DNA samples, of which 926 passed quality control. All samples were subjected to genome-wide SNP genotyping using the Infinium Global Screening Array (GSA) with Shared Custom Content (Illumina Inc.). From these genome-wide data, APOE genotypes were determined either directly (rs7412) or by imputation (rs429358) in all 926 samples. For 80 samples for which no local APOE genotype was available, and for 45 mismatches between locally and GSA derived genotypes (4.8\%), APOE genotype was determined using TaqMan assays (ThermoFisher Scientific, Foster City, CA) on a QuantStudio12K-Flex system in 384-well format. While TaqMan re-genotyping confirmed 23 GSA genotypes and 21 local genotypes, it failed for one sample for which we thus retained the local genotype. For the current study, we classified individuals as APOE $\varepsilon 4$ carriers $(\varepsilon 4+)$ or noncarriers ( $\varepsilon 4-)$ according to their genotype status at rs429358 $(\mathrm{C}$-allele $=\varepsilon 4)$.

\section{Biomarker classifications}

$\mathrm{A} \beta$ status was defined by the CSF $\mathrm{A} \beta_{42 / 40}$, using a cut-off of $<0.063$ to determine abnormality. This cut-off was defined using mixture model analyses in all $\mathrm{AD}$ dementia and $\mathrm{CN}$ individuals of current dataset, ${ }^{29,30}$ which provided a clear binomial distribution (Supplemental Figure 1). Only for the analyses regarding the influence of NFL, Ng and YKL40 on cognition, a median split was used to divide the sample into low and high biomarker values (Cut-off values: NFL: 869 pg/ml; Ng: 103 pg/ml; YKL-40: 163 ng/ml). T-tau status (normal vs. abnormal) was determined using cohort-specific, local cut-off points (Supplemental Table 1). 


\section{Statistical analyses}

Baseline characteristics were compared by $\mathrm{A} \beta$ status and diagnostic group using Chisquare tests for categorical variables and general linear mixed models with study as a random effect for continuous variables. We also tested whether the influence of A $\beta$ on NFL, $\mathrm{Ng}$ and YKL-40 was different across diagnostic groups and age, by examining the diagnostic group by $A \beta$, and age by $A \beta$ interactions. Prior to the comparisons, $A \beta_{42}, N F L, N g$, YKL-40, p-tau and t-tau values were log-transformed to approximate a normal distribution. Spearman's correlations were used to assess the correlations between biomarker values in the total sample and per diagnostic group. General linear mixed models, with random intercepts and slopes by study were used to examine the influence of $A \beta$ status and low/high biomarker levels on MMSE performance and decline over time, adjusted for age, gender, years of education and baseline diagnosis. Missing values for APOE \&4 status $(n=12)$ and years of education $(n=105)$ were imputed using regression analyses within study, based on significant predictors (i.e. age, gender, MMSE, cognitive scores) for these variables. All analyses were repeated after exclusion of individuals with a long interval or missing data on the interval between clinical assessment and CSF collection $(n=23)$. Statistical analyses were performed using R Statistical Software (version 3.3.3) and SPSS (version 24). We used two-sided $\mathrm{p}<0.05$ to define statistical significance. Due to the exploratory nature of the study we did not adjust for multiple comparisons.

\section{RESULTS}

We assessed 770 individuals who were on average 69.3 (SD 8.3) years old and had an average of 10.9 (SD 3.9) years of education. Three hundred ninety-nine (52\%) were female. Clinical follow-up data was available for 557 (73\%) individuals, with an average follow-up length of 2.3 (SD 1.3) years. At baseline 140 (18\%) individuals were considered cognitively normal (CN), 450 (58\%) were diagnosed as having mild cognitive impairment (MCI) and 180 (23\%) as having AD-type dementia.

\section{Demographics and biomarker values}

Table 1 shows the baseline characteristics and biomarker values per diagnostic group, stratified by $A \beta$ status. As expected, in the whole sample, $A \beta+$ individuals were older $(p<0.001)$, more frequently APOE- $\varepsilon 4$ carrier $(\mathrm{p}<0.001)$ and had lower MMSE scores $(\mathrm{p}<0.001)$ compared to $A \beta$ - individuals. When stratified by baseline diagnosis, we found that $A \beta+$ individuals were older compared to the $A \beta$ - individuals in the $\mathrm{CN}(\mathrm{p}<0.001)$ and $\mathrm{MCI}$ $(\mathrm{p}=0.002)$ groups, but not in the AD-type dementia group. Only in MCI we found a dif- 
ference in MMSE score between groups by $A \beta$ status $(p<0.001)$. $A \beta_{38}$ and $A \beta_{40}$ concentrations were lower in the $A \beta+$ group with $A D$-type dementia compared to the $A \beta+C N$ group $\left(A \beta_{38}: p=0.025 ; A \beta_{40}: p=0.036\right)$ and the $A \beta-\left(A \beta_{38}: p=0.007 ; A \beta_{40}: p=0.008\right)$ and $A \beta_{+}\left(A \beta_{38}:\right.$ $\left.\mathrm{p}<0.001 ; \mathrm{A} \beta_{40}: \mathrm{p}=0.001\right) \mathrm{MCI}$ groups. $\mathrm{P}$-tau values were elevated in $\mathrm{A} \beta+$ individuals across all diagnostic groups (all $\mathrm{p}<0.001)$, and increased with disease severity $(\mathrm{p}<0.001)$.

\section{NFL, Ng, YKL-40 and T-tau by A $\beta$ status and baseline diagnosis}

Comparisons by A $\beta$ status and baseline diagnoses of NFL, Ng, YKL-40 and T-tau concentrations are shown in Table 1 and Figure 1. When comparing by $A \beta$ status, NFL and YKL-40 values were differentially increased in $A \beta+C N(N F L: p=0.020, Y K L-40: p=0.001$ ) and MCI individuals (NFL: $\mathrm{p}=0.008$, YKL-40: $\mathrm{p}=0.001$ ), while in the dementia stage NFL and YKL-40 levels were elevated regardless of $A \beta$ status. T-tau and Ng values were stably increased in $A \beta+$ individuals across the cognitive spectrum (T-tau all $p<0.001 ; \mathrm{Ng}$ : $\mathrm{CN}$ : $p=0.005$; MCI: $p<0.001 ; A D p=0.045)$. For NFL we found that the influence of A $\beta$ on NFL was different across diagnoses (interaction $A \beta^{\star}$ diagnosis $\mathrm{p}=0.027$ ). NFL concentrations increased in $\mathrm{A} \beta$ - individuals with advancing clinical stage, while they were stable in the $\mathrm{A} \beta+\mathrm{CN}$ and $\mathrm{MCI}$ groups but increased further in the $\mathrm{A} \beta+\mathrm{AD}$-type dementia group (Figure 1). The influence of $A \beta$ on YKL-40 levels was similar as for NFL (interaction $A \beta^{\star}$ diagnosis $\mathrm{p}=0.001$ ), as the YKL-40 levels in the $A \beta$ - individuals increased with more advanced diagnosis, but remained stable in the $\mathrm{A} \beta+$ individuals (Table 1, Figure 1). For $\mathrm{Ng}$ and $\mathrm{T}$-tau we found that influence of $\mathrm{A} \beta$ was similar across diagnoses (Interaction $\mathrm{A} \beta^{\star} \mathrm{di}$ agnosis T-tau: $\mathrm{p}=0.771 ; \mathrm{Ng}: \mathrm{p}=0.580)$. $\mathrm{A} \beta+$ did have a stronger effect on $\mathrm{Ng}$ and $\mathrm{T}$-tau concentrations in younger individuals than in older individuals (interaction $\mathrm{A} \beta^{\star}$ age $\mathrm{Ng}$ : $\mathrm{p}=0.006$; T-tau: $\mathrm{p}<0.001$ ), while there was no age effect for NFL and YKL-40.

\section{APOE $\varepsilon 4$ carriership}

In $\mathrm{A} \beta$ + individuals, no effect was found of $A P O E \varepsilon 4$ carriership on NFL, Ng and YKL-40 levels, regardless of clinical diagnosis (Table 2). In A $\beta$ - individuals, APOE $\varepsilon 4$ carriership was associated with lower levels of NFL in the total group ( $\mathrm{p}=0.023)$ and in individuals with MCI ( $\mathrm{p}=0.024)$, as well as with lower Ng levels in the MCI ( $\mathrm{p}=0.035)$ and AD-type dementia groups $(\mathrm{p}=0.019)$, but with higher $\mathrm{Ng}$ levels in the total group $(\mathrm{p}=0.008$; Table 2). We found no influence of $A P O E \varepsilon 4$ carriership on YKL-40 and T-tau levels when comparing within $\mathrm{A} \beta$ status, stratified by diagnosis. However, compared to the $\mathrm{CN} A \beta-A P O E$ $\varepsilon 4$ non-carriers, T-tau and YKL-40 levels were elevated in $A \beta+$ individuals regardless of clinical diagnosis (Table 2). 


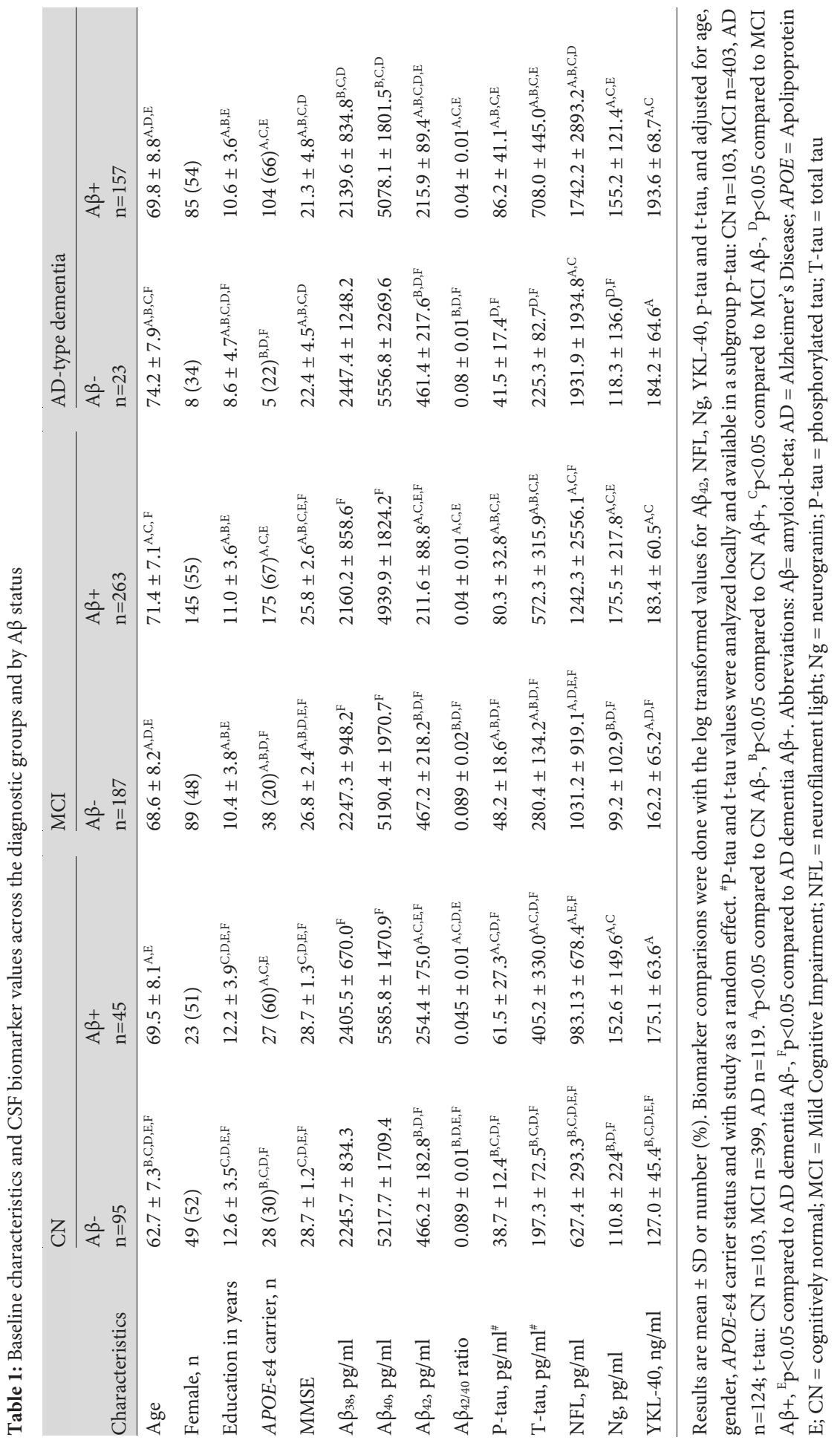




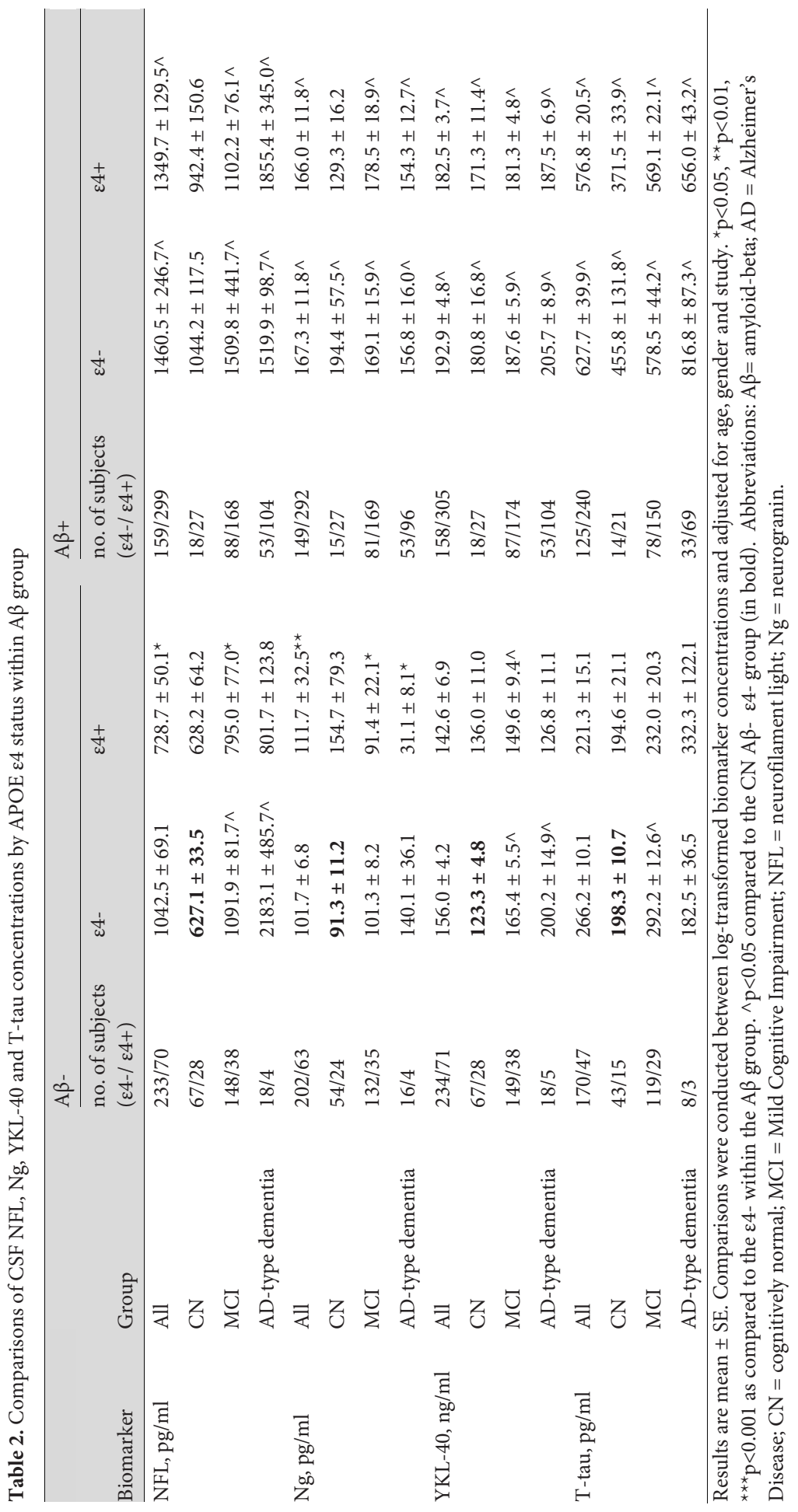


A. Neurofilament light

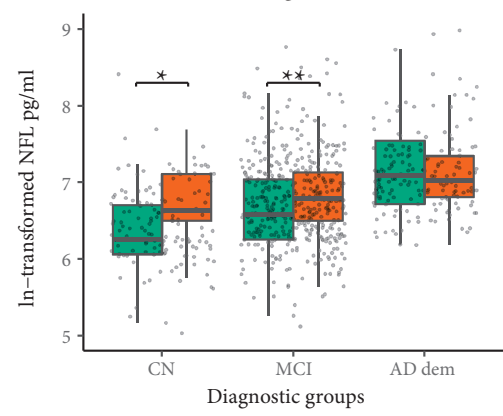

C. YKL-40

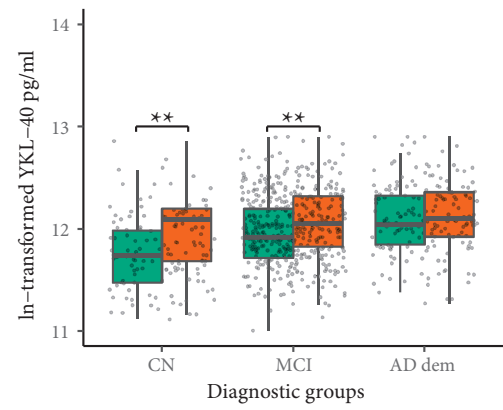

B. Neurogranin

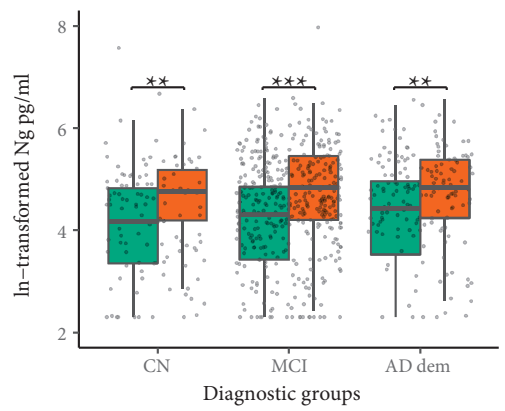

D. T-tau

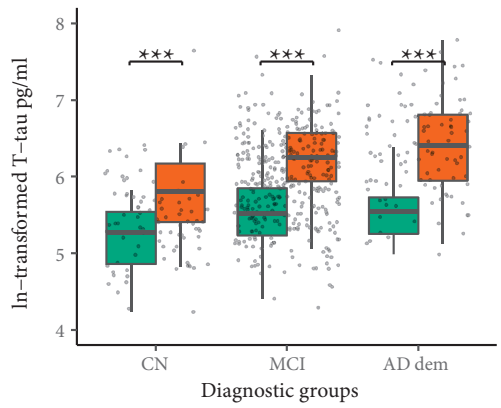

$\mathrm{A} \beta$ status

王 $\mathrm{A} \beta-$ 它 $\mathrm{A} \beta+$

Figure 1. CSF NFL, Ng, YKL-40 and T-tau levels by diagnostic groups and A $\beta$ status

Boxplots (displaying first quartile, median and third quartile) and scatterplots of CSF neurogranin (Ng), neurofilament (NFL) and YKL-40 by diagnostic groups and by $A \beta$ status $\left(A \beta-\right.$ : green; $A \beta+$ : orange). ${ }^{*} p<0.05,{ }^{* *} \mathrm{p}<0.01$, ${ }^{* * *} \mathrm{p}<0.001$ comparisons by $\mathrm{A} \beta$ status within diagnostic group. Figure A shows log transformed NFL concentrations, Figure B shows log transformed Ng concentrations and Figure C shows log transformed YKL-40 concentrations. Figure D shows log transformed T-tau concentrations.

\section{Correlations}

$A \beta_{38}, A \beta_{40}$ and $A \beta_{42}$ were highly positively correlated to each other across all diagnostic groups (Figure 2). In the total sample, a more abnormal $\mathrm{A} \beta_{42 / 40}$ ratio was correlated with higher NFL, Ng and YKL-40 levels in all groups and within each diagnostic group, with a few exceptions (Figure 2). In the $\mathrm{CN}$ group, the $\mathrm{A} \beta_{42 / 40}$ ratio was not correlated with $\mathrm{Ng}$ levels and NFL was not correlated to the $\mathrm{A} \beta_{42 / 40}$ ratio in the AD-type dementia group. Ptau and t-tau were highly correlated, and were both associated with all three emerging biomarkers, in the total group and within each diagnostic group (Figure 2). 

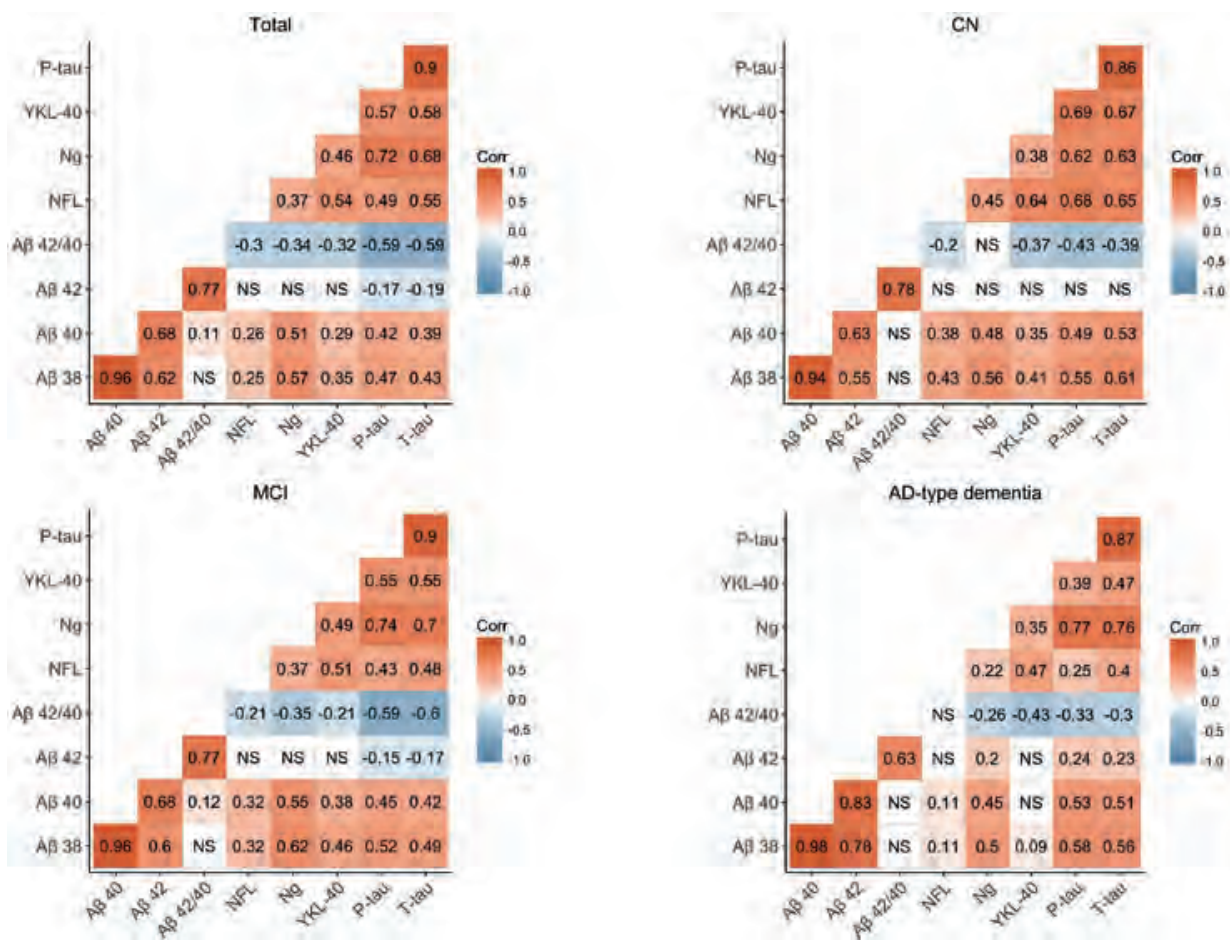

Figure 2. Correlation matrices of CSF biomarkers in the total group and by diagnosis

Correlations between CSF $A \beta_{38}, A \beta_{40}, A \beta_{42}, A \beta_{42 / 40}$, NFL, Ng and YKL-40 levels. Direction of correlation is visualized by color (negative: blue; positive: red) and correlation coefficients are shown for each association. Nonsignificant correlations are visualized with "NS". The top left diagram shows correlations in the total sample, top right in the $\mathrm{CN}$ group, bottom left in the MCI group and bottom right in the AD-type dementia group.

\section{Cognition}

Cross-sectional analysis with MMSE scores as outcome, showed that in the A $\beta+$ individuals high NFL, Ng and T-tau levels were associated with lower MMSE scores in the total group (NFL: $\mathrm{p}<0.001$; Ng: $\mathrm{p}=0.045$; T-tau: $\mathrm{p}<0.001$ ) (Table 3, Figure 3). For NFL, baseline MMSE scores were lower also in the MCI $(\mathrm{p}=0.044)$ and AD-type dementia groups $(\mathrm{p}=0.013)$ and for T-tau in the MCI group $(\mathrm{p}=0.040)$ (Table 3). In A $\beta$ - individuals, high NFL levels were associated with lower MMSE scores in the total group ( $p=0.036)$, and high $\mathrm{T}$-tau levels with lower scores in the AD-type dementia group $(\mathrm{p}=0.031)$. In addition, high Ng levels were associated with higher MMSE scores in the AD-type dementia group $(\mathrm{p}=0.001)$.

Longitudinal analyses with change in MMSE scores as outcome, showed that in A $\beta+$ individuals, high NFL and T-tau levels were associated with an increased rate of decline in the total sample (T-tau: $\mathrm{p}=0.001$; NFL: $\mathrm{p}<0.001$ ). In addition, high NFL and Ng levels were 
also associated with increased rate of decline in the AD-type dementia group (NFL: $\mathrm{p}=0.017$; Ng: $\mathrm{p}=0.001)$. In A $\beta$ - individuals, high NFL, YKL-40 and T-tau levels were associated with an increased rate of decline in the total group (NFL: $\mathrm{p}<0.001$; YKL-40: $\mathrm{p}=0.001$; T-tau: $\mathrm{p}<0.001$ ), as well as in the MCI (NFL: $\mathrm{p}<0.001$; YKL-40: $\mathrm{p}<0.001$; T-tau: $\mathrm{p}<0.001$ ) and AD-type dementia (NFL: $\mathrm{p}=0.027$; YKL-40: $\mathrm{p}=0.017$; T-tau: $\mathrm{p}=0.045$ ) groups (Figure 3). In $\mathrm{A} \beta$ - individuals, high $\mathrm{Ng}$ levels were associated with a decreased rate of decline in the MCI group ( $\mathrm{p}=0.034)$, but with an increased rate of decline in the ADtype dementia group $(\mathrm{p}=0.001)$ (Table 3$)$.
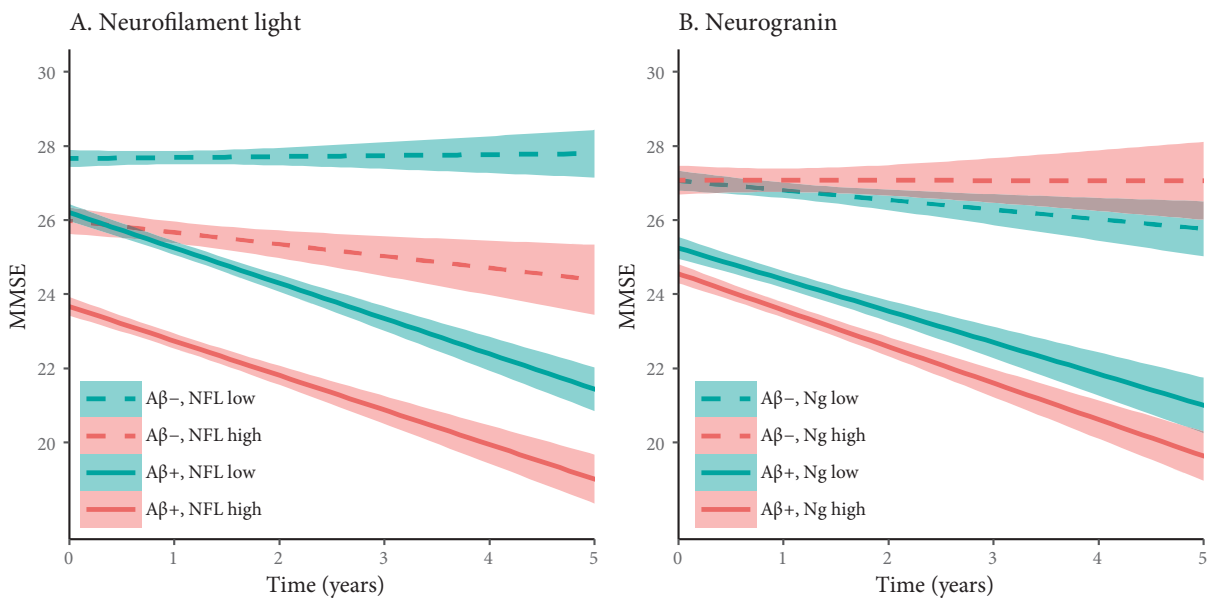

C. YKL -40

D. Total tau
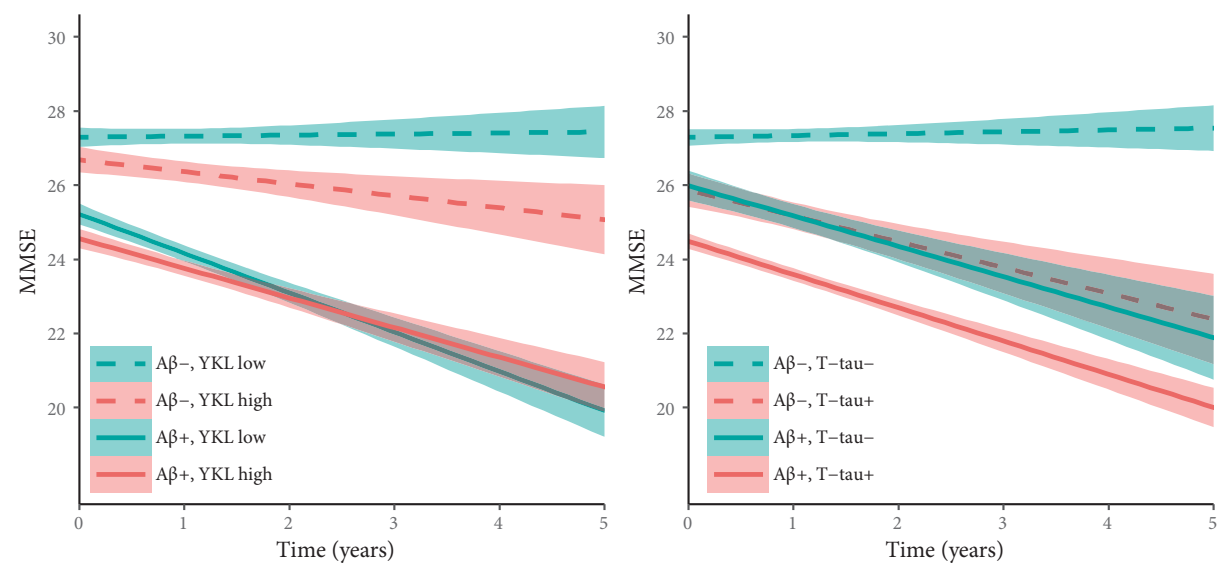

Figure 3. Influence of CSF NFL, Ng, YKL-40 and T-tau on cognition in the total group.

The graphs show mean scores and 95\% confidence intervals of cognitive performance over time for high (red) and low (blue) CSF biomarker levels and by A $\beta$ status (dashed lines: $A \beta$-; solid lines: $A \beta+$ ). Figure A shows the influence of NFL levels. Figure B shows the influence of Ng levels. Figure C shows the influence of YKL-40 levels. Figure D shows the influence of T-tau levels. 
Biomarkers of neurodegeneration, synaptic integrity and astroglial activation

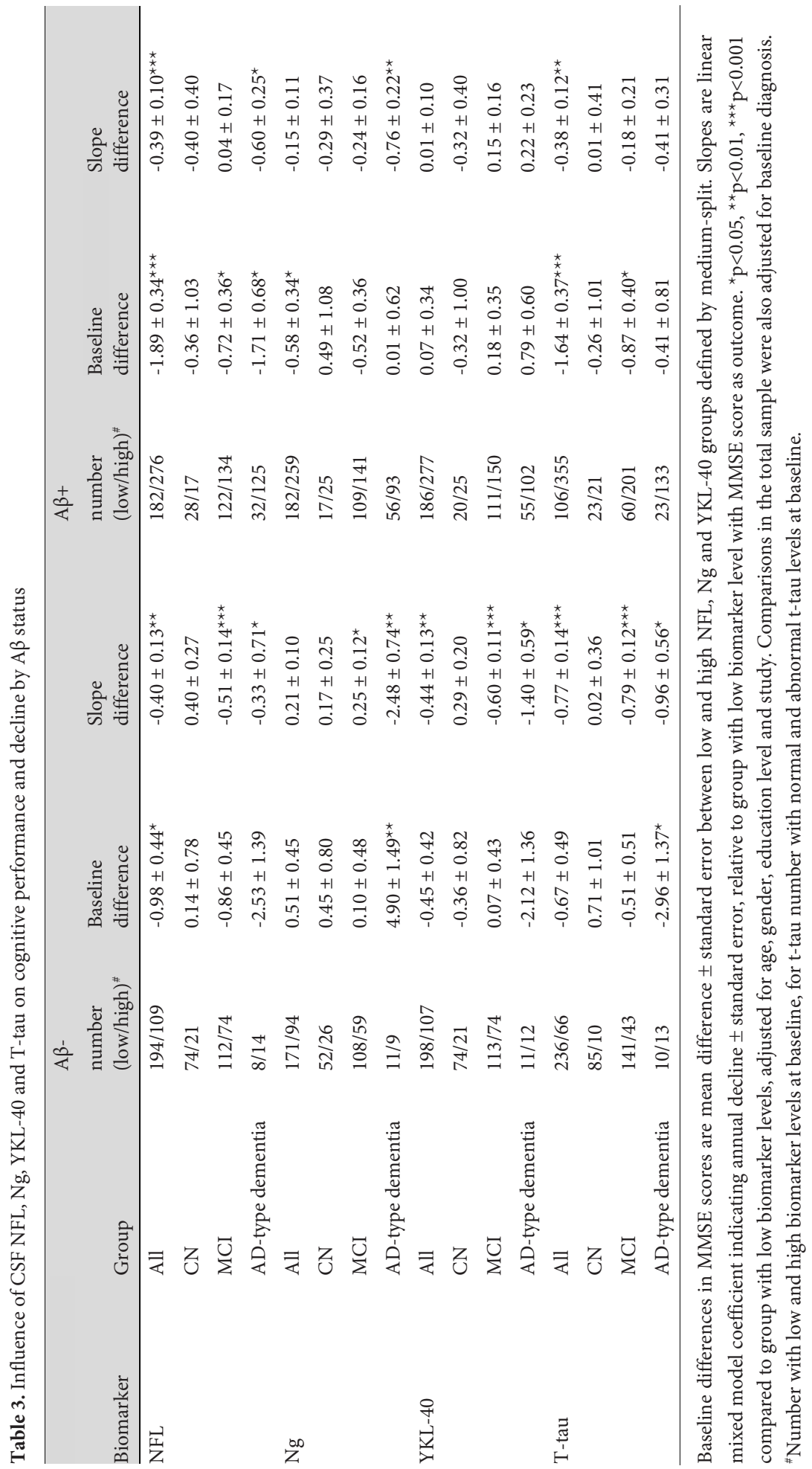


When repeating all analyses without the individuals for whom the interval between CSF collection and cognition was longer than one year or unknown $(n=23)$, results remained similar. Exclusion of one outlier regarding $\mathrm{Ng}$ concentrations, also yielded similar results.

\section{DISCUSSION}

We investigated the relations between $\mathrm{A} \beta$ status, $A P O E \varepsilon 4$ carriership and cognition, with CSF concentrations of NFL, Ng, YKL-40 and T-tau, in a large cohort of individuals across the clinical AD spectrum. The main findings were: (1) CSF NFL, Ng, YKL-40 and T-tau levels were all associated with $A \beta$, and increased with disease severity; (2) A $\beta$ - APOE $\varepsilon 4$ carriers with MCI or AD-type dementia had lower concentrations of NFL and Ng compared to non-carriers; (3) High NFL and T-tau levels were associated with a lower cognitive performance and a faster rate of decline regardless of $A \beta$ status, but predominantly in individuals who were already cognitively impaired; (4) High $\mathrm{Ng}$ levels were associated with lower baseline global cognition scores in $A \beta+$ individuals in the whole sample and high YKL-40 levels were associated with worse global cognitive performance and a faster rate of cognitive decline only in A $\beta$ - individuals with MCI or AD-type dementia.

We found that NFL, Ng, YKL-40 and T-tau concentrations were all associated with A $\beta+$, especially in the preclinical stage. In $A \beta+$ individuals, NFL levels were higher in the dementia stage compared to the MCI stage, whereas Ng and YKL-40 levels stayed relatively stable over time. Yet in A $\beta$ - individuals, we found an increase of both NFL and YKL-40 levels in MCI individuals compared to $\mathrm{CN}$ individuals, while Ng levels in A $\beta$ - individuals remained low with increasing disease severity. T-tau levels increased with disease severity regardless of $A \beta$ status, albeit the rate of increase was faster in $A \beta+$ individuals. These findings confirm that synaptic dysfunction - as measured by $\mathrm{Ng}$ - plays an important role in $\mathrm{AD}$ pathophysiology in all clinical stages. ${ }^{31,32}$ In addition, our data verifies that axonal degeneration and neuroinflammation - as respectively measured by NFL and YKL-40 are less specific to $\mathrm{AD},{ }^{8,9}$ but their temporal pattern across the clinical stages is $\mathrm{AD}$ specific: in AD, NFL and YKL-40 levels are already increased in the preclinical stage, while in non- $\mathrm{AD}$ (i.e. in $\mathrm{A} \beta$ - individuals) concentrations merely start to increase from the MCI stage onwards. Our findings regarding T-tau levels, confirm the role of altered neuronal tau metabolism in $\mathrm{AD},{ }^{33,34}$ and support the notion this process also occur in other conditions (i.e. in $A \beta$ - individuals), although to a lesser extent. ${ }^{35}$ Together these results provide novel insights into the temporal pattern of AD pathophysiology, which should be validated by longitudinal biomarker studies. 
The APOE \&4 allele is the strongest genetic risk factor for $\mathrm{AD}$, but how this allele affects $\mathrm{AD}$ is still unclear. ${ }^{36} \mathrm{We}$ found that in $\mathrm{A} \beta+$ individuals, NFL, Ng, YKL-40 and T-tau levels did not differ between $A P O E \varepsilon 4$ carriers and non-carriers in all clinical stages, suggesting that these markers reflect a generic reaction to amyloid aggregation regardless of $A P O E$ genotype. In A $\beta$ - individuals, APOE $\varepsilon 4$ non-carriers with MCI or AD-type dementia had higher NFL and Ng levels compared to carriers, and in case of NFL even comparable levels to $A \beta+A P O E \& 4$ carriers and non-carriers from the same clinical group. This suggests that the $\mathrm{A} \beta-A P O E \varepsilon 4$ non-carriers with $\mathrm{MCI}$ or $\mathrm{AD}$-type dementia might have other pathologies not related to $\mathrm{A} \beta$ and $A P O E \varepsilon 4$ carriership that are causing cognitive impairment and axonal degeneration, and to a lesser extend also synaptic dysfunction, causing an AD-like clinical presentation. Regarding T-tau and YKL-40 levels, we found similar concentrations in APOE \&4 carriers and non-carriers, which is in line with previous studies, ${ }^{37-39}$ but in contrast with a previous study in which a modest association of APOE $\varepsilon 4$ carriership on YKL-40 levels was found in individuals with MCI due to AD. ${ }^{40}$ Besides the inconsistency with the latter study, possibly due to heterogeneity in sample sizes or biomarker classifications, our results confirm that YKL-40 concentrations are independent of $A P O E \varepsilon 4$ carriership.

Higher levels of NFL and T-tau were both associated with a lower cognitive performance at baseline and increased rate of decline regardless of A $\beta$ status. As both NFL and T-tau are markers of axonal degeneration, ${ }^{12,41}$ these findings imply that axonal loss is an important driver of cognitive decline in both $\mathrm{AD}$ and other neurodegenerative processes. ${ }^{9,42}$ Concerning $\mathrm{Ng}$, we found that only in individuals with AD-type dementia, higher concentrations were also associated with a faster rate of decline, regardless of $A \beta$. This is congruent with previous studies suggesting that synaptic loss might be strongly associated with cognition, irrespective of amyloid plaque pathology. ${ }^{42-44}$ However, synaptic dysfunction was also found to be associated with cognitive decline in preclinical $\mathrm{AD},{ }^{11}$ a finding we could not confirm with our analyses, possibly due to a lower sensitivity of the cognitive outcome measure we used (i.e. MMSE). Posthoc, we explored this notion by repeating the analyses in a subgroup ( $\mathrm{n}=615)$ with memory performance as an outcome, and found that high $\mathrm{Ng}$ levels tended to be associated with a faster decline in memory performance in CN $A \beta+(\beta=-0.24, p=0.052)$, but not in CN A $\beta$ - individuals $(\beta=-0.10, p=0.462$; data not shown). The negative impact of high YKL-40 levels on cognition seems to only relate to $\mathrm{A} \beta$ - individuals. This implies that, in individuals who do not have amyloid pathology and clinically present with MCI or AD-type dementia, astroglial activation may represent a major underlying mechanism and may have a profound effect on cognitive decline. This makes YKL-40 an important prognostic marker for individuals with MCI but without evidence of $A \beta$ pathology, for instance those with Suspected Non-Alzheimer's Disease 
Pathophysiology (SNAP); a biomarker-based subtype of individuals with AD-like neurodegeneration but without evidence of amyloid pathology. ${ }^{45}$

Strengths of this study are the large sample size of individuals across the AD spectrum, the central analyses of various CSF biomarkers and the availability of clinical follow-up data. Nonetheless, there are also some limitations that should be mentioned. First, the data was collected at different centers using routine local protocols. However, the CSF samples were analyzed centrally for most outcome measures - $A \beta_{38}, A \beta_{40}, A \beta_{42}, N F L, N g$ and YKL-40 - and clinical data was harmonized using validated methods like standardization and dichotomization. Second, our AD-type dementia group contained A $\beta$ - individuals; a consequence of only using a clinical diagnosis to classify this group, instead of a biomarker-based diagnosis. Although this makes our demented group more heterogeneous, it does reflect current clinical practice. Third, our clinical follow-up may have been too short to get an accurate view of cognitive trajectories over time. And lastly, we chose the MMSE to assess cognition as this was available in nearly all individuals, but it might not be sensitive enough to detect subtle cognitive decline and decline in specific cognitive domains. Future studies with longer follow-up and other cognitive measures should therefore validate our results regarding cognitive decline.

In conclusion, we found that NFL, Ng and YKL-40 were associated with A $\beta$ pathology, showing that axonal degeneration, synaptic dysfunction and neuroinflammation are all to some extent involved in AD pathophysiology. Furthermore, we found that NFL is a generic prognostic marker which is elevated early in $\mathrm{AD}$, and has a profound influence on baseline cognition and decline over time. $\mathrm{Ng}$ is a useful $\mathrm{AD}$ marker as it is closely related to $A \beta$ and tau in all cognitive stages and is associated with cognition. Moreover, $\mathrm{Ng}$ may be a useful maker to detect the effectiveness of synaptic therapy, a potential disease modifying therapy for $\mathrm{AD} .{ }^{46,47} \mathrm{YKL}-40$ has a profound influence on cognitive decline in absence of $A \beta$, and thereby may be of value to increase the accuracy of the prognosis of individuals with SNAP. These findings improve prognostic accuracy and increase our knowledge of the mechanisms underlying AD. 


\section{REFERENCES}

1. Zetterberg H. Applying fluid biomarkers to Alzheimer's disease. Am J Physiol Cell Physiol. 2017;313(1):C3C10.

2. Albert MS, DeKosky ST, Dickson D, et al. The diagnosis of mild cognitive impairment due to Alzheimer's disease: recommendations from the National Institute on Aging-Alzheimer's Association workgroups on diagnostic guidelines for Alzheimer's disease. Alzheimer's \& dementia : the journal of the Alzheimer's Association. 2011;7(3):270-279.

3. Blennow K, Mattsson N, Scholl M, Hansson O, Zetterberg H. Amyloid biomarkers in Alzheimer's disease. Trends Pharmacol Sci. 2015;36(5):297-309.

4. Lewczuk P, Matzen A, Blennow K, et al. Cerebrospinal Fluid Abeta42/40 Corresponds Better than Abeta42 to Amyloid PET in Alzheimer's Disease. Journal of Alzheimer's disease : JAD. 2017;55(2):813-822.

5. Tsai M, Tangalos EG, Petersen RC, et al. Apolipoprotein E: risk factor for Alzheimer disease. American journal of human genetics. 1994;54(4):643.

6. Zetterberg H, Skillback T, Mattsson N, et al. Association of Cerebrospinal Fluid Neurofilament Light Concentration With Alzheimer Disease Progression. JAMA Neurol. 2016;73(1):60-67.

7. Bruno D, Pomara N, Nierenberg J, et al. Levels of cerebrospinal fluid neurofilament light protein in healthy elderly vary as a function of TOMM40 variants. Experimental gerontology. 2012;47(5):347-352.

8. Antonell A, Mansilla A, Rami L, et al. Cerebrospinal fluid level of YKL-40 protein in preclinical and prodromal Alzheimer's disease. Journal of Alzheimer's disease : JAD. 2014;42(3):901-908.

9. Skillback T, Farahmand B, Bartlett JW, et al. CSF neurofilament light differs in neurodegenerative diseases and predicts severity and survival. Neurology. 2014;83(21):1945-1953.

10. Portelius E, Zetterberg H, Skillback T, et al. Cerebrospinal fluid neurogranin: relation to cognition and neurodegeneration in Alzheimer's disease. Brain : a journal of neurology. 2015;138(Pt 11):3373-3385.

11. Kester MI, Teunissen CE, Crimmins DL, et al. Neurogranin as a Cerebrospinal Fluid Biomarker for Synaptic Loss in Symptomatic Alzheimer Disease. JAMA Neurol. 2015;72(11):1275-1280.

12. Zetterberg H, Skillback T, Mattsson N, et al. Association of Cerebrospinal Fluid Neurofilament Light Concentration With Alzheimer Disease Progression. JAMA Neurol. 2016;73(1):60-67.

13. Sala-Llonch R, Idland AV, Borza T, et al. Inflammation, Amyloid, and Atrophy in The Aging Brain: Relationships with Longitudinal Changes in Cognition. Journal of Alzheimer's disease : JAD. 2017;58(3):829-840.

14. Janelidze S, Hertze J, Zetterberg H, et al. Cerebrospinal fluid neurogranin and YKL-40 as biomarkers of Alzheimer's disease. Ann Clin Transl Neurol. 2016;3(1):12-20.

15. Lista S, Toschi N, Baldacci F, et al. Diagnostic accuracy of CSF neurofilament light chain protein in the biomarker-guided classification system for Alzheimer's disease. Neurochem Int. 2017;108:355-360.

16. Thorsell A, Bjerke M, Gobom J, et al. Neurogranin in cerebrospinal fluid as a marker of synaptic degeneration in Alzheimer's disease. Brain research. 2010;1362:13-22.

17. Bos I, Vos, S.J.B., Vandenberghe, R., Scheltens, P., Engelborghs, S., Frisoni, G., Molinuevo, J., Wallin, A., Lléo, A., Popp, J., Martinez-Lage, P., Baird, A., Dobson, R., Legido-Quigley, C., Bertram, L., Sleegers, K., Kate ten, M., Barkhof, F., Zetterberg, H., Lovestone, S., Streffer, J., Visser, P. . The EMIF-AD Multimodal Biomarker Discovery Study: Design, methods and cohort characteristics. Submitted.

18. Visser PJ, Verhey FR, Boada M, et al. Development of screening guidelines and clinical criteria for predementia Alzheimer's disease. The DESCRIPA Study. Neuroepidemiology. 2008;30(4):254-265.

19. Reijs BLR, Ramakers I, Elias-Sonnenschein L, et al. Relation of Odor Identification with Alzheimer's Disease Markers in Cerebrospinal Fluid and Cognition. Journal of Alzheimer's disease : JAD. 2017;60(3):1025-1034. 
20. Galluzzi S, Marizzoni M, Babiloni C, et al. Clinical and biomarker profiling of prodromal Alzheimer's disease in workpackage 5 of the Innovative Medicines Initiative PharmaCog project: a 'European ADNI study'. Journal of internal medicine. 2016;279(6):576-591.

21. van der Flier WM, Pijnenburg YA, Prins N, et al. Optimizing patient care and research: the Amsterdam Dementia Cohort. Journal of Alzheimer's disease : JAD. 2014;41(1):313-327.

22. Somers C, Struyfs H, Goossens J, et al. A Decade of Cerebrospinal Fluid Biomarkers for Alzheimer's Disease in Belgium. Journal of Alzheimer's disease : JAD. 2016;54(1):383-395.

23. Estanga A, Ecay-Torres M, Ibanez A, et al. Beneficial effect of bilingualism on Alzheimer's disease CSF biomarkers and cognition. Neurobiology of aging. 2017;50:144-151.

24. Tautvydaite D, Kukreja D, Antonietti JP, Henry H, von Gunten A, Popp J. Interaction between personality traits and cerebrospinal fluid biomarkers of Alzheimer's disease pathology modulates cognitive performance. Alzheimer's research \& therapy. 2017;9(1):6.

25. Petersen RC. Mild cognitive impairment as a diagnostic entity. Journal of internal medicine. 2004;256(3):183-194.

26. McKhann G, Drachman D, Folstein M, Katzman R, Price D, Stadlan EM. Clinical diagnosis of Alzheimer's disease: report of the NINCDS-ADRDA Work Group under the auspices of Department of Health and Human Services Task Force on Alzheimer's Disease. Neurology. 1984;34(7):939-944.

27. Folstein MF, Folstein SE, McHugh PR. "Mini-mental state". A practical method for grading the cognitive state of patients for the clinician. Journal of psychiatric research. 1975;12(3):189-198.

28. Olsson B, Hertze J, Lautner R, et al. Microglial markers are elevated in the prodromal phase of Alzheimer's disease and vascular dementia. Journal of Alzheimer's disease : JAD. 2013;33(1):45-53.

29. Benaglia T, Chauveau D, Hunter D, Young D. mixtools: An R package for analyzing finite mixture models. Journal of Statistical Software. 2009;32(6):1-29.

30. Palmqvist $\mathrm{S}$, Zetterberg $\mathrm{H}$, Blennow $\mathrm{K}$, et al. Accuracy of brain amyloid detection in clinical practice using cerebrospinal fluid $\beta$-amyloid 42: a cross-validation study against amyloid positron emission tomography. JAMA neurology. 2014;71(10):1282-1289.

31. Selkoe DJ. Alzheimer's disease is a synaptic failure. Science. 2002;298(5594):789-791.

32. Musardo S, Marcello E. Synaptic dysfunction in Alzheimer's disease: From the role of amyloid beta-peptide to the alpha-secretase ADAM10. European journal of pharmacology. 2017;817:30-37.

33. Hampel H, Blennow K, Shaw LM, Hoessler YC, Zetterberg H, Trojanowski JQ. Total and phosphorylated tau protein as biological markers of Alzheimer's disease. Experimental gerontology. 2010;45(1):30-40.

34. Selkoe DJ. The molecular pathology of Alzheimer's disease. Neuron. 1991;6(4):487-498.

35. Iqbal K, Alonso Adel C, Chen S, et al. Tau pathology in Alzheimer disease and other tauopathies. Biochimica et biophysica acta. 2005;1739(2-3):198-210.

36. Liu CC, Liu CC, Kanekiyo T, Xu H, Bu G. Apolipoprotein E and Alzheimer disease: risk, mechanisms and therapy. Nat Rev Neurol. 2013;9(2):106-118.

37. Craig-Schapiro R, Perrin RJ, Roe CM, et al. YKL-40: a novel prognostic fluid biomarker for preclinical Alzheimer's disease. Biological psychiatry. 2010;68(10):903-912.

38. Sutphen CL, Jasielec MS, Shah AR, et al. Longitudinal Cerebrospinal Fluid Biomarker Changes in Preclinical Alzheimer Disease During Middle Age. JAMA Neurol. 2015;72(9):1029-1042.

39. Morris JC, Roe CM, Xiong C, et al. APOE predicts amyloid-beta but not tau Alzheimer pathology in cognitively normal aging. Annals of neurology. 2010;67(1):122-131.

40. Gispert JD, Monte GC, Suarez-Calvet M, et al. The APOE epsilon4 genotype modulates CSF YKL-40 levels and their structural brain correlates in the continuum of Alzheimer's disease but not those of sTREM2. Alzheimers Dement (Amst). 2017;6:50-59.

41. Blennow K, Wallin A, Agren H, Spenger C, Siegfried J, Vanmechelen E. Tau protein in cerebrospinal fluid: a biochemical marker for axonal degeneration in Alzheimer disease? Molecular and chemical neuropathology. 1995;26(3):231-245. 
42. Kvartsberg H, Duits FH, Ingelsson M, et al. Cerebrospinal fluid levels of the synaptic protein neurogranin correlates with cognitive decline in prodromal Alzheimer's disease. Alzheimer's \& dementia : the journal of the Alzheimer's Association. 2015;11(10):1180-1190.

43. Masliah E, Mallory M, Alford M, et al. Altered expression of synaptic proteins occurs early during progression of Alzheimer's disease. Neurology. 2001;56(1):127-129.

44. Mattsson N, Insel PS, Palmqvist S, et al. Cerebrospinal fluid tau, neurogranin, and neurofilament light in Alzheimer's disease. EMBO Mol Med. 2016;8(10):1184-1196.

45. Jack CR, Jr., Knopman DS, Chetelat G, et al. Suspected non-Alzheimer disease pathophysiology--concept and controversy. Nat Rev Neurol. 2016;12(2):117-124.

46. Nistico R, Pignatelli M, Piccinin S, Mercuri NB, Collingridge G. Targeting synaptic dysfunction in Alzheimer's disease therapy. Mol Neurobiol. 2012;46(3):572-587.

47. Teich AF, Nicholls RE, Puzzo D, et al. Synaptic therapy in Alzheimer's disease: a CREB-centric approach. Neurotherapeutics. 2015;12(1):29-41. 


\section{SUPPLEMENTAL DATA}

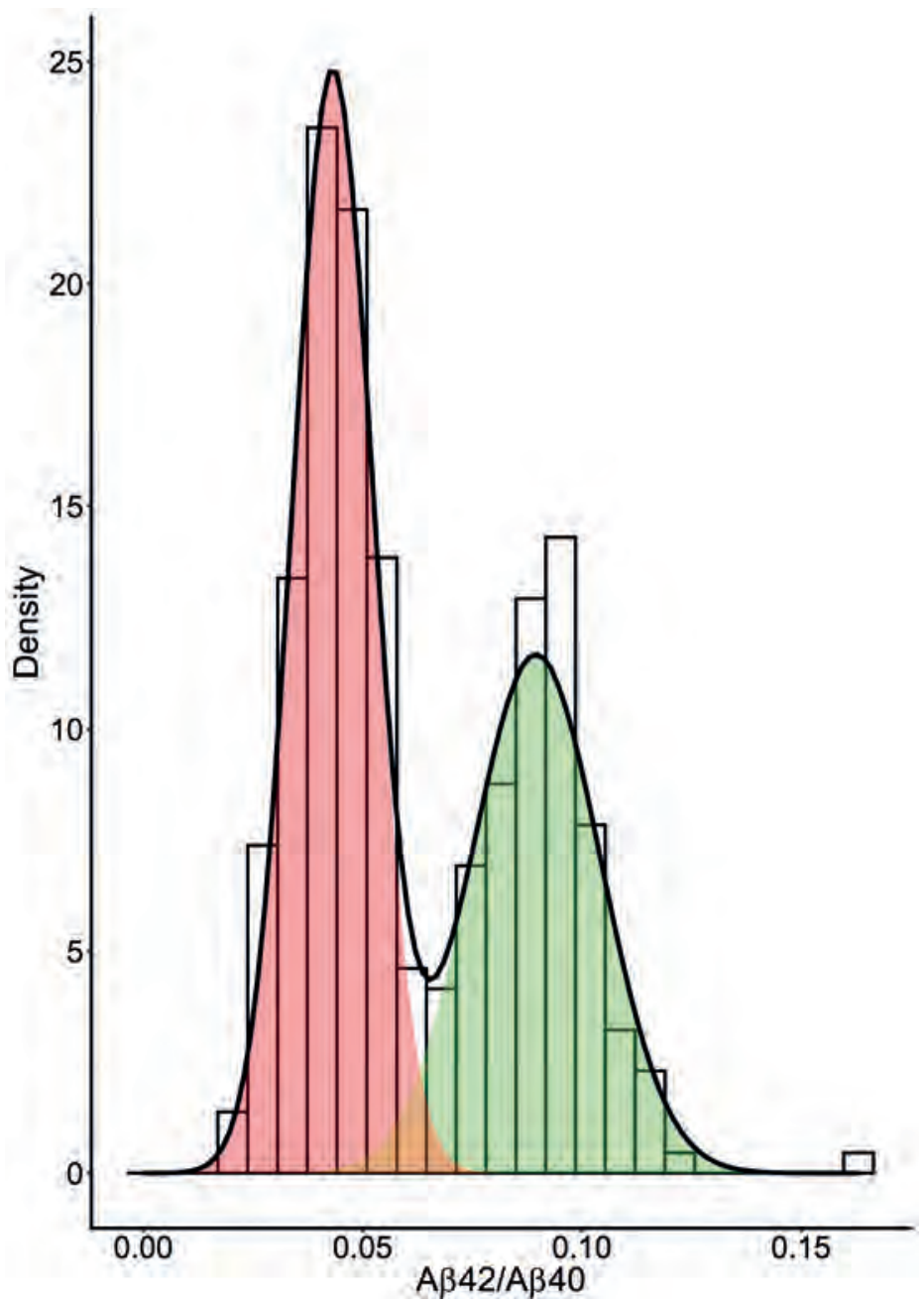

Supplemental Figure 1. Density plot of $A \beta_{42 / 40}$ ratio

Distribution of $A \beta_{42 / 40}$ values based on mixture model analyses. Dashed lines represents the cut-point $(0.063)$ in binominal distribution. 



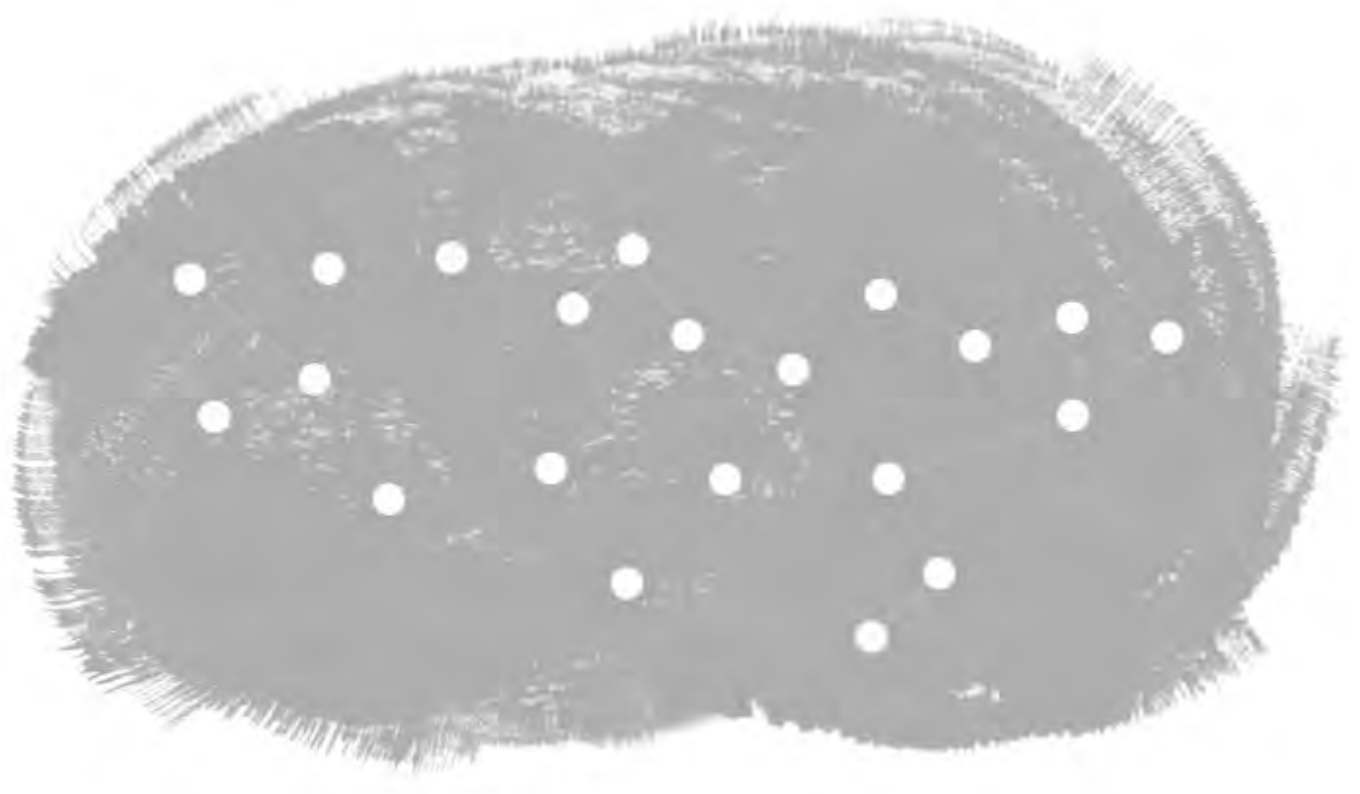




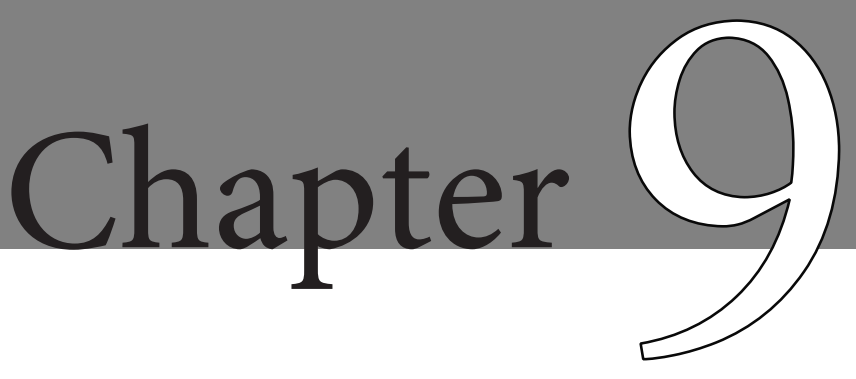

Increased cerebrospinal fluid amyloid- $\beta 38$ and 40 concentrations in individuals with mild cognitive impairment and Suspected Non-Alzheimer's Disease Pathophysiology

Submitted

Stephanie J. B. Vos, Isabelle Bos, Frans Verhey, Sebastiaan Engelborghs, Alberto Lleó, Peter Johannsen, Yvonne Freund-Levi, Lutz Froelich, Rik Vandenberghe, Giovanni Frisoni, Olivier Blin, Jill Richardson, Régis Bordet, Philip Scheltens, Inez Ramakers, Julius Popp, Magda Tsolaki, Pablo Martinez-Lage, Maria Bjerke, Charlotte Teunissen, Gwendoline Peyratout, Mikel Tainta, Valerija Dobricic, Lars Bertram, Simon Lovestone, Johannes Streffer, Kaj Blennow, Ulf Andreasson, Henrik Zetterberg, Pieter Jelle Visser 


\section{ABSTRACT}

Background: Individuals with Alzheimer's disease (AD)-like neuronal injury but without evidence of amyloid pathology are referred to as having Suspected Non-Alzheimer's Disease Pathophysiology (SNAP). To date it is unclear what the biology is underlying this biomarker profile in individuals with mild cognitive impairment (MCI).

Methods: We included 496 individuals with MCI and 88 normal cognition (CN). Based on amyloid status (A+/-), defined by abnormal CSF amyloid- $\beta(\mathrm{A} \beta) 1-42$, and tau status $(\mathrm{T}+/-)$ status, defined by either abnormal total tau ( $\mathrm{t}$-tau) or phosphorylated tau ( $\mathrm{p}$-tau), individuals with MCI were classified in four groups: A-T-, A+T-A-T+ (SNAP), A+T+. $\mathrm{CN} A-\mathrm{T}$ - individuals served as a reference group. We compared CSF measures of A $\beta 1$ 38, A $\beta 1-40, A \beta 1-42$, t-tau, p-tau, neurogranin (Ng, synaptic marker), neurofilament-light (NFL, axonal degeneration marker), and YKL-40 (astrocytes activation marker) between groups and performed unbiased cluster analysis based on CSF markers in MCI-SNAP to define SNAP subgroups.

Results: 584 individuals were included (mean age 68.6 (SD 8.4) years, 54\% female). MCISNAP had $~ 1.5$ times higher CSF A $\beta 38$ and A $\beta 40$ levels compared to all other groups and higher NFL, Ng, and YKL-40 levels compared to all other groups, except MCI A+T+. Cluster analyses identified three MCI-SNAP subgroups. Cluster A (20\%) differed from the other MCI-SNAP groups by high $\mathrm{A} \beta 42$ levels, a high $\mathrm{A} \beta 42 / \mathrm{A} \beta 40$ ratio, and less APOE 44 carriers. Cluster B (65\%) had lower A $\beta 38, A \beta 40, N F L, N g$, YKL-40, t-tau and ptau levels than other MCI-SNAP groups, but higher levels than CN A-T-. The A $\beta 42 / A \beta 40$ ratio was lower than cluster A, but higher than cluster C. Cluster C (14\%) had a lower $\mathrm{A} \beta 42 / \mathrm{A} \beta 40$ ratio and more APOE\&4 carriers than other MCI-SNAP groups, similar to $\mathrm{MCI} \mathrm{A}+\mathrm{T}+$.

Conclusions: Compared to controls, MCI-SNAP is characterized by increased CSF levels of $\mathrm{A} \beta 38, \mathrm{~A} \beta 40$ and biomarkers reflecting axonal loss, synaptic dysfunction, and astrocyte activation. MCI-SNAP is etiologically heterogeneous and consists of several subgroups, including a small subgroup with a low $\mathrm{A} \beta 42 / \mathrm{A} \beta 40$ ratio, suggestive of $\mathrm{AD}$. Our results are the first step towards a positive definition of non-amyloid pathology in MCI-SNAP. 


\section{INTRODUCTION}

Individuals with a biomarker profile reflecting Alzheimer's disease (AD)-like neuronal injury but without evidence of amyloid pathology are referred to as having Suspected Non-Alzheimer's Disease Pathophysiology (SNAP). ${ }^{1}$ Previously, we found that a relatively large group of individuals with mild cognitive impairment (MCI) have SNAP (29\%) and that a substantial number of these individuals still progress to AD-type dementia in the short-term (25\%). ${ }^{2}$ To date it is unknown whether SNAP is an atypical form of AD, non-AD neurodegeneration, early $\mathrm{AD}$, or consists of multiple subgroups with different pathologies.

SNAP can be defined in cerebrospinal fluid (CSF) by normal amyloid-beta (A $\beta) 42$ levels and abnormal levels of total tau ( $\mathrm{t}$-tau) or phosphorylated tau ( $\mathrm{p}$-tau) or on imaging by normal A $\beta$ positron emission tomography (PET) scan and abnormal tau PET scan, fluodeoxyglucose (FDG) PET scan, or hippocampal atrophy. Studies on MCI-SNAP have shown a lower frequency of apolipoprotein $(A P O E) \varepsilon 4$ alleles ${ }^{2-5}$ and some indicate a male predominance $^{3-5}$ but not all. ${ }^{2}$ One possible explanation for the phenomenon of MCISNAP is that it reflects some individuals having particularly high pre-onset A $\beta 42$ levels, in which case a decrease as seen in AD would not be apparent. Since A $\beta 40$ and A $\beta 38$ levels more closely reflect total $A \beta$ production and do not decrease as a consequence of $A D$ pathology, ${ }^{6}$ relating $\mathrm{A} \beta 42$ to these levels may be a better measure to detect an $\mathrm{AD}$-related decrease in $A \beta 42$ in individuals with high premorbid levels. In addition, CSF markers for axonal degeneration (neurofilament-light, NFL), ${ }^{7}$ synaptic dysfunction (neurogranin, $\mathrm{Ng}$ ), ${ }^{8}$ and astrocyte activation (YKL-40) ${ }^{9}$ may further help to characterize MCI-SNAP.

In the current study we aimed to investigate the underlying pathophysiology of MCISNAP using CSF targeted proteomics in a uniquely large multicenter cohort. We measured different CSF A $\beta$ isoforms ( $A \beta 38, A \beta 40, A \beta 42)$, NFL, $N g$, and YKL-40 in individuals with MCI-SNAP and compared these to MCI individuals with other biomarker profiles as well as to cognitively normal $(\mathrm{CN})$ individuals. In addition, we investigated whether MCI-SNAP subgroups can be identified based on CSF protein profiles.

\section{METHODS}

\section{Participants}

The European Medical Information Framework (IMI-EMIF; http://www.emif.eu/) is a public-private pre-competitive platform designed to enable the visibility and facilitate the reuse of pre-existing data for research. Using this platform we identified and obtained 
samples from multiple research cohorts across Europe for the EMIF-AD Multimodal Biomarker Discovery study ${ }^{10}$ and MCI-SNAP study. We recruited individuals from five European single center studies. These included the Amsterdam dementia cohort, ${ }^{11}$ Antwerp memory clinic cohort, ${ }^{12}$ Gipuzkoa Alzheimer Project (GAP) study from San Sebastian, ${ }^{13}$ Lausanne memory clinic cohort, ${ }^{14}$ and Maastricht memory clinic cohort; ${ }^{15}$ and from three European multicenter studies: DESCRIPA, ${ }^{16}$ EDAR, ${ }^{17}$ and Pharmacog ${ }^{18}$. Inclusion criteria were $\mathrm{CN}$ individuals or individuals with diagnosis of $\mathrm{MCI},{ }^{19}$ with baseline data of CSF ttau and p-tau levels, and availability of CSF samples for central analyses. Based on CSF A $\beta 42$ and tau markers we selected 5 groups as described below. All individuals underwent a cognitive assessment including the mini-mental state examination (MMSE) and routine neuropsychological testing.

\section{APOE genotyping}

APOE genotyping was performed in 90\% $(\mathrm{n}=527)$ of the individuals. For 476 individuals, $A P O E$ genotype was available from central genetic analyses which were conducted in 345 DNA and 132 blood samples, from which DNA was extracted using QIAamp DNA Blood Mini Kit (QIAGEN GmbH, Hilden, Germany). All samples were subjected to genome-wide SNP genotyping using the Infinium Global Screening Array (GSA) with Shared Custom Content (Illumina Inc.). From these genome-wide data, APOE genotypes were determined either directly (rs7412) or by imputation (rs429358). For samples for which no local $A P O E$ genotype was available or for mismatches between locally and GSA derived genotypes (4.8\%), APOE genotype was determined using TaqMan assays (ThermoFisher Scientific, Foster City, CA) on a QuantStudio-12K-Flex system in 384-well format. For 51 samples for which no DNA or whole blood was contributed for central analyses, the local genotypes were used. For the current study, we classified 527 individuals as $A P O E \varepsilon 4$ carriers $(\varepsilon 4+)$ or non-carriers ( $\varepsilon 4-)$ according to their genotype status at rs429358 (C-allele $=\varepsilon 4)$.

\section{CSF analyses}

CSF was collected by a lumbar puncture and thereafter centrifuged and stored at $-80^{\circ} \mathrm{C}$ in polypropylene tubes. T-tau and p-tau were measured locally according to clinical laboratory practice derived using INNOTEST ELISAs (Fujirebio, Ghent, Belgium). For a subset of the EDAR study, these CSF measures were analyzed using Alzbio3 xMAP Luminex $(n=106)$. For all 548 individuals, CSF samples were shipped on dry ice to the Neurochemistry Lab of University of Gothenburg in Mölndal, Sweden, where central analysis of $\mathrm{A} \beta 38, \mathrm{~A} \beta 40, \mathrm{~A} \beta 42, \mathrm{NFL}, \mathrm{Ng}$, and YKL-40 levels was performed. $\mathrm{A} \beta 38, \mathrm{~A} \beta 40$, and $\mathrm{A} \beta 42$ were measured using the V-PLEX Plus A $\beta$ Peptide Panel 1 (6E10) Kit from Meso Scale Discovery (MSD, Rockville, MD). NFL concentration was measured using a commercial 
ELISA (NF-light ${ }^{\circledast}$ ELISA, UmanDiagnostics, Umeå, Sweden). ${ }^{20} \mathrm{Ng}$ was measured using an in-house immunoassay for Ng. ${ }^{21}$ YKL-40 was determined by a human chitinase-3 quantikine ELISA kit (R\&D systems, Inc, Minneapolis, MN). ${ }^{22}$ All analyses were performed according to the manufacturer's instructions in one occasion and using one batch of reagents (except for 12 EDAR cases for which CSF was already analysed in Gothenburg) by board-certified laboratory technicians who were blinded to clinical information. The value $10 \mathrm{pg} / \mathrm{mL}$ was used for $\mathrm{Ng}$ values below the detection limit. For YKL-40 values above the highest detectable value, the highest measurable value was used. NFL values were missing for 8 cases and $\mathrm{Ng}$ values for 7 cases due to low sample volume.

\section{Participant classifications}

Individuals were classified in biomarker groups based on the presence of amyloid $(\mathrm{A}+)$ and tau $(\mathrm{T}+)$ pathophysiology as CN A-T-, MCI A-T-, MCI A+T-, MCI A-T+ (SNAP) and MCI A+T+. A+ was defined as CSF A 342 levels below $328 \mathrm{pg} / \mathrm{ml}$, a cutoff based on mixture modeling in all $\mathrm{AD}$ dementia and $\mathrm{CN}$ individuals of the current cohort distribution (data not shown). T+ was defined as either abnormal CSF t-tau or abnormal CSF ptau, based on study specific cut-offs (Supplementary Table 1). In secondary analyses, A+ was defined as CSF $A \beta 42 / A \beta 40$ ratio lower than 0.063 , a cut-off based on mixture modeling in the current cohort, as described above.

\section{Statistical analyses}

We compared demographics and CSF measures between $\mathrm{CN}$ and MCI biomarker groups using Chi-square tests for categorical variables and general linear mixed models (GLMM) with study as a random effect for continuous variables with Bonferroni correction for multiple comparisons and adjusted for age and sex. All CSF results were log-transformed in order to normalize the distribution, but the untransformed values are presented in the tables. We performed K-means clustering analyses to identify biomarker-based subgroups in MCI-SNAP based on all available CSF analytes. Five cases were excluded from these analyses because of unavailability of some of the CSF measures. Chi-square tests for categorical variables and GLMM with study as a random effect for continuous variables with Bonferroni correction for multiple comparisons and adjustment for age and sex were used to compare CSF measures between cluster groups and other biomarker groups. Spearman's rho correlations were performed to investigate the relationship between CSF measures in MCI and MCI-SNAP. Statistical packages SPSS (version 22.0) and R (version 3.3.3) were used with two-sided statistical significance set at $\mathrm{P}<0.05$. 


\section{RESULTS}

\section{Sample characteristics}

We included 584 individuals with a mean age of 68.6 (SD 8.4) years, of which 317 (54\%) were female and 87 had MCI-SNAP. Sample characteristics per biomarker and clinical group are presented in Table 1. Individuals with MCI-SNAP were older than the CN and MCI biomarker negative groups (A-T-), had a lower MMSE score than the CN A-Tgroup but a higher score than the MCI A+T+ group, and were less often APOE $\varepsilon 4$ carrier than the $\mathrm{A}+\mathrm{T}+$ group.

\section{CSF biomarker levels in MCI-SNAP}

MCI-SNAP had 1.5 times higher CSF A $\beta 38$ and A $\beta 40$ levels compared to all other groups (Table 1, Figure 1). As expected based on the classifications used, A $\beta 42$ levels were higher compared to A+T- and A+T+groups, and similar to A-T-groups. The A $\beta 42 / A \beta 40$ ratio in the MCI-SNAP group was also higher compared to $\mathrm{A}+\mathrm{T}$ - and $\mathrm{A}+\mathrm{T}+$ groups, but lower compared to A-T- groups. MCI-SNAP showed, by definition, higher t-tau and $\mathrm{p}$ tau levels compared to A-T- and A+T-groups, and similar levels compared to the A+T+ group. Individuals with MCI-SNAP had higher NFL, Ng and YKL-40 levels compared to $\mathrm{A}-\mathrm{T}$ - and A+T-groups, and similar levels as the A+T+group (Table 1, Figure 1).

Within the MCI-SNAP group 32 individuals (37\%) had an abnormal A $\beta 42 / A \beta 40$ ratio. Individuals with MCI-SNAP and an abnormal A $\beta 42 / \mathrm{A} \beta 40$ ratio were more often $A P O E$ $\varepsilon 4$ carriers, had lower $A \beta 42$ levels, and higher $\mathrm{Ng}$, t-tau and $\mathrm{p}$-tau levels compared to individuals with MCI-SNAP and a normal A $\beta 42 / \mathrm{A} \beta 40$ ratio. These findings and the relation to CSF values in the other groups are described in Supplemental Table 2.

\section{CSF biomarker subgroups in MCI-SNAP}

The cluster analyses identified three MCI-SNAP subgroups: Cluster A ( $n=17,20 \%$ of MCI-SNAP group), Cluster B ( $\mathrm{n}=55,65 \%$ of MCI-SNAP group), and Cluster C ( $\mathrm{n}=12$, $14 \%$ of MCI-SNAP group). Characteristics of these three MCI-SNAP subgroups in relation to the other groups are presented in Tables 2 and 3, and Figure 2. In summary, MCISNAP Cluster A differed from the other MCI-SNAP groups by high A $\beta 42$ levels, a high $\mathrm{A} \beta 42 / \mathrm{A} \beta 40$ ratio, and low frequency of $A P O E \varepsilon 4$ carriership. Cluster $\mathrm{B}$ was characterized by lower levels of A $\beta 38, A \beta 40, N F L, N g, Y K L-40$, t-tau and p-tau compared to the other MCI-SNAP subgroups, although levels were higher than in the CN A-T-group. The $\mathrm{A} \beta 42 / \mathrm{A} \beta 40$ ratio in cluster $\mathrm{B}$ was lower compared to cluster $\mathrm{A}$, but higher compared to cluster $\mathrm{C}$. The frequency of APOE $\varepsilon 4$ carriers in cluster B was lower compared to MCISNAP cluster C, although it was higher than in the CN A-T-group. Cluster C had a lower 
$\mathrm{A} \beta 42 / \mathrm{A} \beta 40$ ratio and higher frequency of $A P O E \varepsilon 4$ carriers (73\% vs. $19-42 \%)$ than the other MCI-SNAP subgroups, similar to the A+T+group.

Table 1: Sample characteristics and CSF values by biomarker group

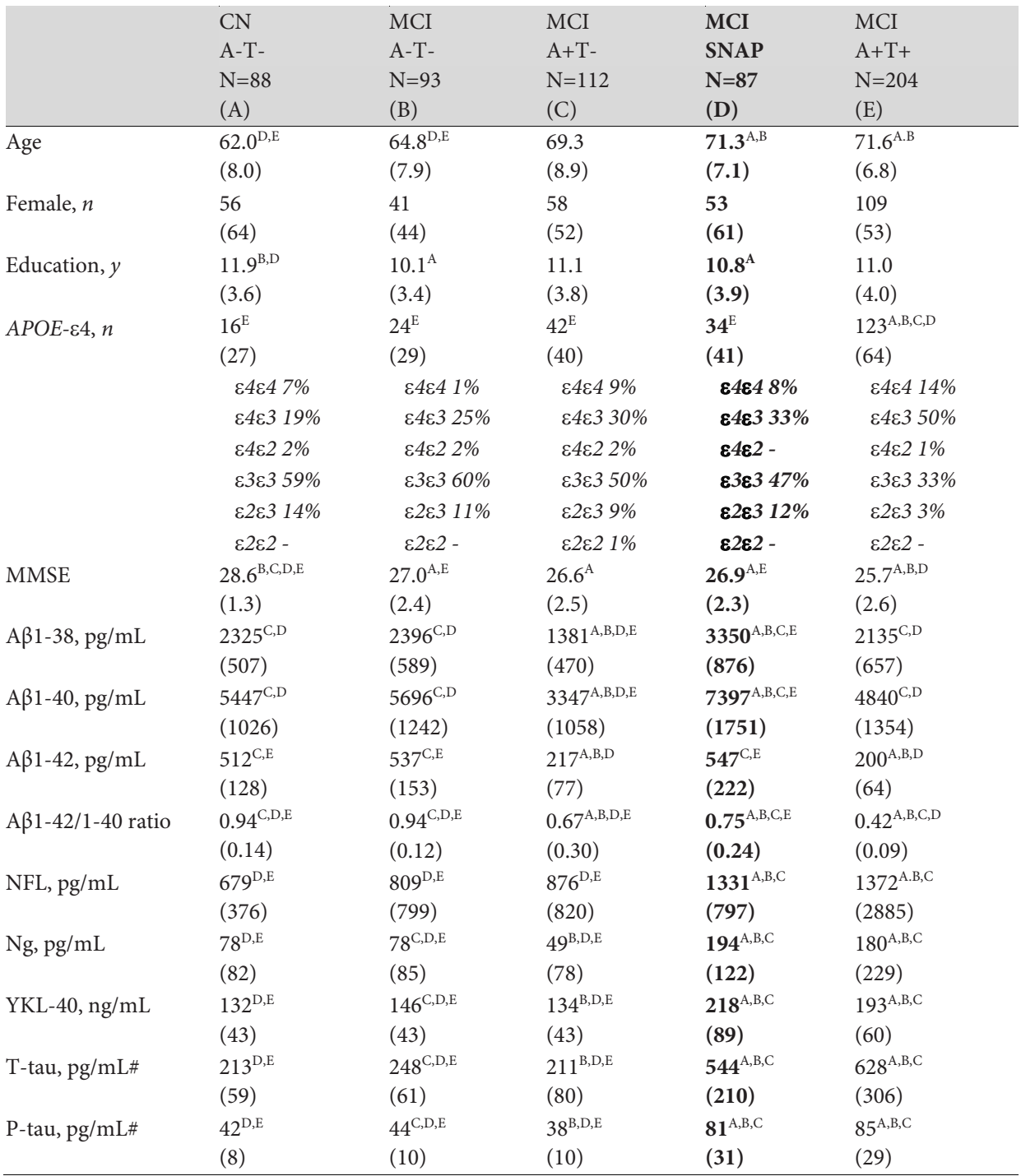

Results are means (SD) or numbers (\%). ${ }^{\mathrm{A}} \mathrm{P}<0.05$ compared to $\mathrm{CN} \mathrm{A}-\mathrm{T}$-; ${ }^{\mathrm{B}} \mathrm{P}<0.05$ compared to $\mathrm{MCI} \mathrm{A}-\mathrm{T}$-; ${ }^{\mathrm{C}} \mathrm{P}<0.05$ compared to MCI A+T-; ${ }^{\mathrm{D}} \mathrm{P}<0.05$ compared to $\mathrm{MCI} \mathrm{A}-\mathrm{T}+(\mathrm{SNAP}) ;{ }^{\mathrm{E}} \mathrm{P}<0.05$ compared to $\mathrm{MCI} \mathrm{A}+\mathrm{T}+$; CSF values were log transformed to test statistical differences between groups. \#Center measured values using ELISA; some EDAR cases were not included here as another CSF assay was used. The A $\beta 1-42 / A \beta 1-40$ ratio was multiplied by 10 . $\mathrm{A} \beta=$ beta amyloid; $\mathrm{AD}=$ Alzheimer's disease; $\mathrm{APOE}=$ apolipoprotein $\mathrm{E}$; $\mathrm{CN}=$ cognitively normal; $\mathrm{MCI}=$ mild cognitive impairment; NFL=neurofilament-light, Ng=neurogranin; T-tau=total tau; Ptau=phosphorylated tau. 
A

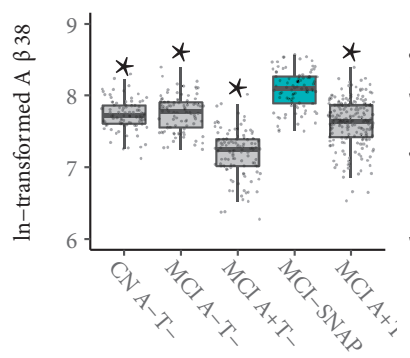

$\mathrm{D}$

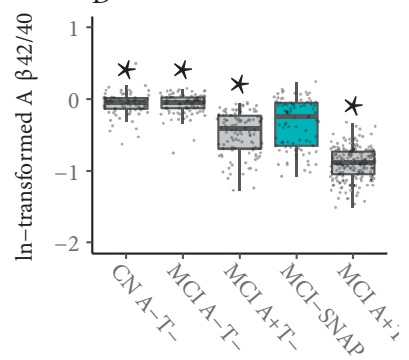

G

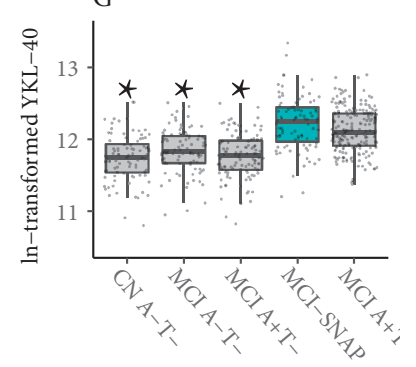

B

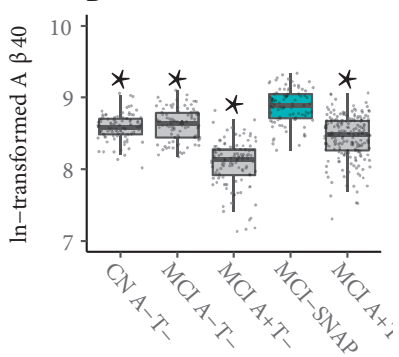

E

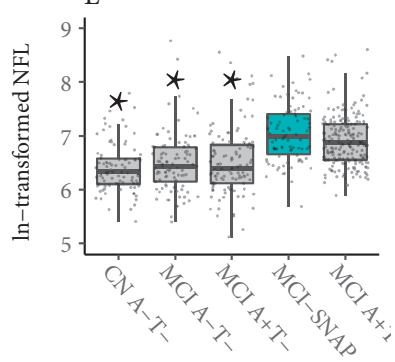

$\mathrm{H}$

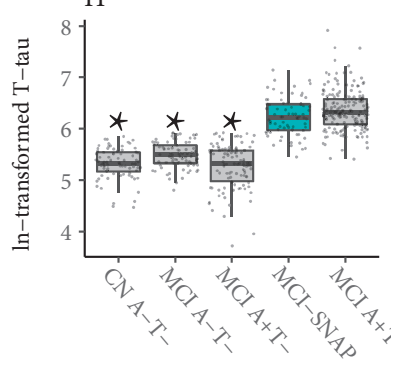

C

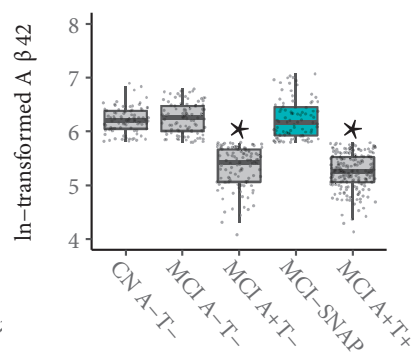

$\mathrm{F}$
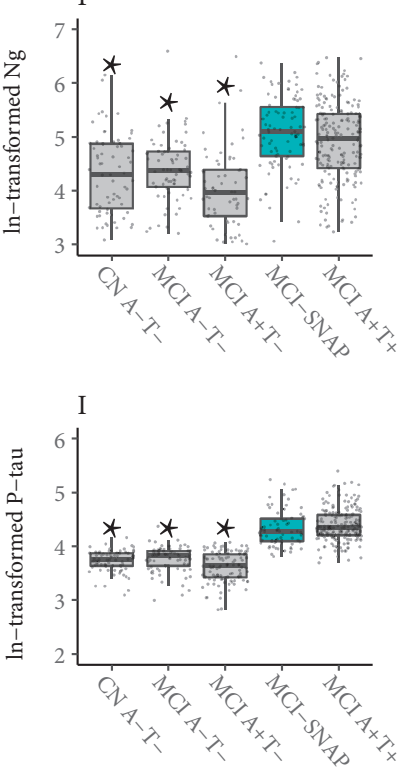

Figure 1. CSF values by biomarker group

Results are boxplots (displaying first quartile, median and third quartile) and scatterplots for all CSF measures by each biomarker subgroup: (A) amyloid- $\beta 38$, (B) amyloid- $\beta 40$, (C) amyloid- $\beta 42$, (D) amyloid- $\beta 42 / 40$, (E) Neurofilament-light, (F) Neurogranin, (G) YKL-40, (H) total tau, (I) phosphorylated tau. The MCI-SNAP group is presented in blue and the $\mathrm{CN}$ A-T-, $\mathrm{MCI} \mathrm{A}-\mathrm{T}-, \mathrm{MCI} \mathrm{A}+\mathrm{T}-$-, and $\mathrm{MCI} \mathrm{A}+\mathrm{T}$ groups are presented in grey. CSF biomarker values were logtransformed. "Indicates statistically significant differences compared to MCISNAP. $\mathrm{A}=$ amyloid $-\beta 42, \mathrm{~T}=$ total or phosphorylated tau, $\mathrm{CN}=$ cognitively normal, $\mathrm{MCI}=$ mild cognitive impairment. 

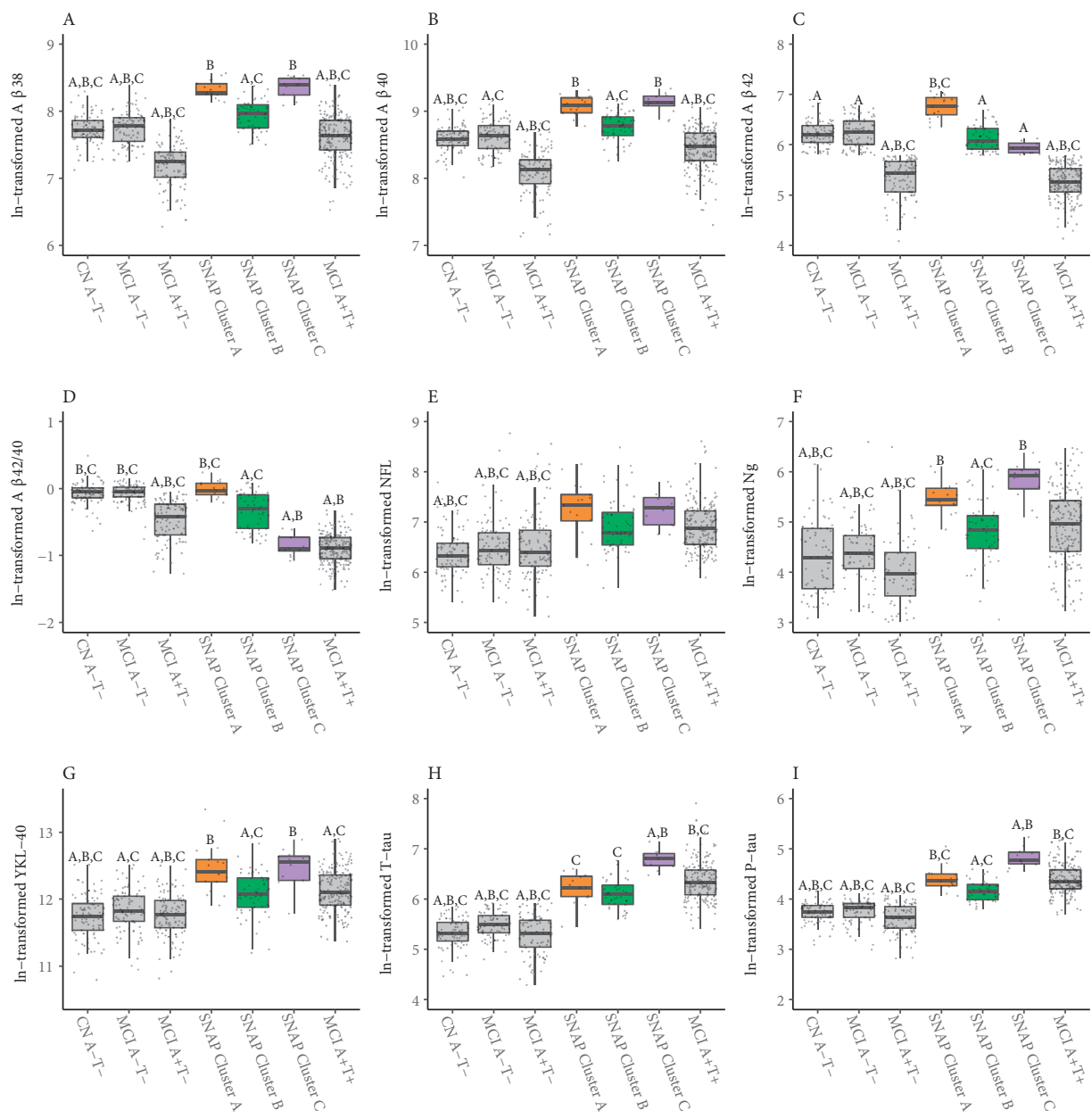

Figure 2. CSF values for MCI A-T+ clusters compared to other MCI biomarker groups

Results are boxplots (displaying first quartile, median and third quartile) and scatterplots for all CSF measures by each MCI A-T+ subgroup and other MCI biomarker subgroups: (A) amyloid- $\beta 38$, (B) amyloid- $\beta 40,(\mathrm{C})$ amyloid- $\beta 42$, (D) amyloid- $\beta 42 / 40$, (E) Neurofilament-light, (F) Neurogranin, (G) YKL-40, (H) total tau, (I) phosphorylated tau. MCI A-T+Cluster A is presented in orange, MCI A-T+ Cluster B in green, MCI A-T+ Cluster $\mathrm{C}$ purple and MCI A-T-, MCI A+T-, and MCI A+T+ groups are presented in grey. ' $\mathrm{A}$ ' indicates statistically significant differences compared to MCI A-T+Cluster A; 'B' compared to MCI A-T+Cluster B, and 'C' compared to MCI A-T+ Cluster C. CSF biomarker values were log transformed. $A=$ amyloid $-\beta$ 1-42, $T=$ total or phosphorylated tau, $\mathrm{CN}=$ cognitively normal, $\mathrm{MCI}=$ mild cognitive impairment. 
Table 2: CSF subgroups in MCI A-T+

\begin{tabular}{|c|c|c|c|c|c|c|}
\hline & $\begin{array}{l}\text { MCI A-T+ } \\
\text { Cluster A } \\
\mathrm{N}=17\end{array}$ & $\begin{array}{l}\text { MCI A-T+ } \\
\text { Cluster B } \\
\mathrm{N}=55\end{array}$ & $\begin{array}{l}\text { MCI A-T+ } \\
\text { Cluster C } \\
\mathrm{N}=12\end{array}$ & $\begin{array}{l}\text { Cluster A } \\
\text { comparisons to } \\
\text { other groups }\end{array}$ & $\begin{array}{l}\text { Cluster B } \\
\text { comparisons to } \\
\text { other groups }\end{array}$ & $\begin{array}{l}\text { Cluster C } \\
\text { comparisons to } \\
\text { other groups }\end{array}$ \\
\hline Age & $71.0(7.0)$ & $71.4(7.2)$ & $71.9(7.4)$ & $>\mathrm{CN}$ & $\begin{array}{l}>\mathrm{CN} \\
>\mathrm{MCI} \text { A-T- }\end{array}$ & $>\mathrm{CN}$ \\
\hline Female, $n$ & $9(53)$ & $35(64)$ & $7(58)$ & - & - & - \\
\hline Education, $y$ & $11.6(3.2)$ & $10.7(4.3)$ & $10.6(2.7)$ & - & - & - \\
\hline$A P O E-\varepsilon 4, n$ & $\begin{array}{l}3(18)^{\mathrm{C}} \\
\varepsilon 4 \varepsilon 318 \% \\
\varepsilon 3 \varepsilon 363 \% \\
\varepsilon 2 \varepsilon 319 \%\end{array}$ & $\begin{array}{l}22(42)^{\mathrm{C}} \\
\varepsilon 4 \varepsilon 48 \% \\
\varepsilon 4 \varepsilon 334 \% \\
\varepsilon 3 \varepsilon 349 \% \\
\varepsilon 2 \varepsilon 39 \%\end{array}$ & $\begin{array}{l}8(73)^{\mathrm{AB}} \\
\varepsilon 4 \varepsilon 418 \% \\
\varepsilon 4 \varepsilon 355 \% \\
\varepsilon 3 \varepsilon 327 \%\end{array}$ & $<\mathrm{MCI} A+\mathrm{T}+$ & - & - \\
\hline MMSE & $\begin{array}{l}27.5 \\
(1.7)\end{array}$ & $\begin{array}{l}27.1 \\
(2.3)\end{array}$ & $\begin{array}{l}25.6 \\
(3.1)\end{array}$ & - & - & $<\mathrm{CN}$ \\
\hline$A \beta 1-38, p g / m L$ & $\begin{array}{l}4162^{\mathrm{B}} \\
(549)\end{array}$ & $\begin{array}{l}2883^{\mathrm{AC}} \\
(621)\end{array}$ & $\begin{array}{l}4318^{\mathrm{B}} \\
(644)\end{array}$ & $\begin{array}{l}>\mathrm{CN} \\
>\mathrm{MCI} A-\mathrm{T}- \\
>\mathrm{MCI} \mathrm{A}+\mathrm{T}- \\
>\mathrm{MCI} \mathrm{A}+\mathrm{T}+\end{array}$ & $\begin{array}{l}>\mathrm{CN} \\
>\mathrm{MCI} A-\mathrm{T}- \\
>\mathrm{MCI} \mathrm{A}+\mathrm{T}- \\
>\mathrm{MCI} \mathrm{A}+\mathrm{T}+\end{array}$ & $\begin{array}{l}>\mathrm{CN} \\
>\mathrm{MCI} A-\mathrm{T}- \\
>\mathrm{MCI} \mathrm{A}+\mathrm{T}- \\
>\mathrm{MCI} \mathrm{A}+\mathrm{T}+\end{array}$ \\
\hline$A \beta 1-40, p g / m L$ & $\begin{array}{l}8884^{\mathrm{B}} \\
(1257)\end{array}$ & $\begin{array}{l}6504^{\mathrm{AC}} \\
(1274)\end{array}$ & $\begin{array}{l}9248^{\mathrm{B}} \\
(1330)\end{array}$ & $\begin{array}{l}>\mathrm{CN} \\
>\mathrm{MCI} A-\mathrm{T}- \\
>\mathrm{MCI} \mathrm{A}+\mathrm{T}- \\
>\mathrm{MCI} \mathrm{A}+\mathrm{T}+\end{array}$ & $\begin{array}{l}>\mathrm{CN} \\
>\mathrm{MCI} \mathrm{A}+\mathrm{T}- \\
>\mathrm{MCI} \mathrm{A}+\mathrm{T}+\end{array}$ & $\begin{array}{l}>\mathrm{CN} \\
>\mathrm{MCI} \text { A-T- } \\
>\mathrm{MCI} \mathrm{A+T-} \\
>\mathrm{MCI} \mathrm{A}+\mathrm{T}+\end{array}$ \\
\hline$A \beta 1-42, p g / m L$ & $\begin{array}{l}885^{\mathrm{BC}} \\
(189)\end{array}$ & $\begin{array}{l}471^{\mathrm{A}} \\
(120)\end{array}$ & $\begin{array}{l}394^{\mathrm{A}} \\
(74)\end{array}$ & $\begin{array}{l}>\mathrm{CN} \\
>\mathrm{MCI} \text { A-T- } \\
>\mathrm{MCI} \mathrm{A}+\mathrm{T}- \\
>\mathrm{MCI} \mathrm{A}+\mathrm{T}+\end{array}$ & $\begin{array}{l}>\mathrm{MCI} A+\mathrm{T}- \\
>\mathrm{MCI} \mathrm{A}+\mathrm{T}+\end{array}$ & $\begin{array}{l}>\mathrm{MCI} A+\mathrm{T}- \\
>\mathrm{MCI} \mathrm{A}+\mathrm{T}+\end{array}$ \\
\hline $\begin{array}{l}A \beta 1-42 / A \beta 1-40 \\
\text { ratio }\end{array}$ & $\begin{array}{l}0.99^{\mathrm{BC}} \\
(0.12)\end{array}$ & $\begin{array}{l}0.74^{\mathrm{AC}} \\
(0.20)\end{array}$ & $\begin{array}{l}0.43^{\mathrm{AB}} \\
(0.07)\end{array}$ & $\begin{array}{l}>\mathrm{MCI} A+\mathrm{T}- \\
>\mathrm{MCI} \mathrm{A}+\mathrm{T}+\end{array}$ & $\begin{array}{l}<\mathrm{CN} \\
<\mathrm{MCI} A-\mathrm{T}- \\
>\mathrm{MCI} A+\mathrm{T}- \\
>\mathrm{MCI} \mathrm{A}+\mathrm{T}+\end{array}$ & $\begin{array}{l}<\mathrm{CN} \\
<\mathrm{MCI} A-\mathrm{T}- \\
<\mathrm{MCI} \text { A+T- }\end{array}$ \\
\hline $\mathrm{NFL}, \mathrm{pg} / \mathrm{mL}$ & $\begin{array}{l}1595 \\
(833)\end{array}$ & $\begin{array}{l}1199 \\
(822)\end{array}$ & $\begin{array}{l}1480 \\
(520)\end{array}$ & $\begin{array}{l}>\mathrm{CN} \\
>\mathrm{MCI} A-\mathrm{T}- \\
>\mathrm{MCI} \mathrm{A}+\mathrm{T}-\end{array}$ & $\begin{array}{l}>\mathrm{CN} \\
>\mathrm{MCI} A-\mathrm{T}- \\
>\mathrm{MCI} \mathrm{A}+\mathrm{T}-\end{array}$ & $\begin{array}{l}>\mathrm{CN} \\
>\mathrm{MCI} A-\mathrm{T}- \\
>\mathrm{MCI} \text { A+T- }\end{array}$ \\
\hline $\mathrm{Ng}, \mathrm{pg} / \mathrm{mL}$ & $\begin{array}{l}246^{\mathrm{B}} \\
(90)\end{array}$ & $\begin{array}{l}138^{\mathrm{AC}} \\
(84)\end{array}$ & $\begin{array}{l}362^{\mathrm{B}} \\
(122)\end{array}$ & $\begin{array}{l}>\mathrm{CN} \\
>\mathrm{MCI} A-\mathrm{T}- \\
>\mathrm{MCI} \mathrm{A}+\mathrm{T}-\end{array}$ & $\begin{array}{l}>\mathrm{CN} \\
>\mathrm{MCI} A-\mathrm{T}- \\
>\mathrm{MCI} \mathrm{A}+\mathrm{T}-\end{array}$ & $\begin{array}{l}>\mathrm{CN} \\
>\mathrm{MCI} A-\mathrm{T}- \\
>\mathrm{MCI} \mathrm{A}+\mathrm{T}- \\
>\mathrm{MCI} \mathrm{A}+\mathrm{T}+\end{array}$ \\
\hline YKL-40, ng/mL & $\begin{array}{l}280^{\mathrm{B}} \\
(125)\end{array}$ & $\begin{array}{l}186^{\mathrm{AC}} \\
(59)\end{array}$ & $\begin{array}{l}268^{\mathrm{B}} \\
(72)\end{array}$ & $\begin{array}{l}>\mathrm{CN} \\
>\mathrm{MCI} A-\mathrm{T}- \\
>\mathrm{MCI} \mathrm{A}+\mathrm{T}- \\
>\mathrm{MCI} \mathrm{A}+\mathrm{T}+\end{array}$ & $\begin{array}{l}>\mathrm{CN} \\
>\mathrm{MCI} \mathrm{A}+\mathrm{T}-\end{array}$ & $\begin{array}{l}>\mathrm{CN} \\
>\mathrm{MCI} A-\mathrm{T}- \\
>\mathrm{MCI} \mathrm{A}+\mathrm{T}- \\
>\mathrm{MCI} \mathrm{A}+\mathrm{T}+\end{array}$ \\
\hline T-tau, pg/mL\# & $\begin{array}{l}521^{\mathrm{C}} \\
(141)\end{array}$ & $\begin{array}{l}463^{\mathrm{C}} \\
(141)\end{array}$ & $\begin{array}{l}897^{\mathrm{AB}} \\
(175)\end{array}$ & $\begin{array}{l}>\mathrm{CN} \\
>\mathrm{MCI} A-\mathrm{T}- \\
>\mathrm{MCI} \mathrm{A}+\mathrm{T}-\end{array}$ & $\begin{array}{l}>\mathrm{CN} \\
>\mathrm{MCI} A-\mathrm{T}- \\
>\mathrm{MCI} \mathrm{A}+\mathrm{T}- \\
<\mathrm{MCI} \mathrm{A+T}+\end{array}$ & $\begin{array}{l}>\mathrm{CN} \\
>\mathrm{MCI} A-\mathrm{T}- \\
>\mathrm{MCI} \mathrm{A}+\mathrm{T}- \\
>\mathrm{MCI} \mathrm{A}+\mathrm{T}+\end{array}$ \\
\hline P-tau, pg/mL\# & $\begin{array}{l}84^{\mathrm{BC}} \\
(24)\end{array}$ & $\begin{array}{l}66^{\mathrm{AC}} \\
(15)\end{array}$ & $\begin{array}{l}128^{\mathrm{AB}} \\
(27)\end{array}$ & $\begin{array}{l}>\mathrm{CN} \\
>\mathrm{MCI} A-\mathrm{T}- \\
>\mathrm{MCI} \mathrm{A}+\mathrm{T}-\end{array}$ & $\begin{array}{l}>\mathrm{CN} \\
>\mathrm{MCI} A-\mathrm{T}- \\
>\mathrm{MCI} \mathrm{A}+\mathrm{T}- \\
<\mathrm{MCI} \mathrm{A+T}+\end{array}$ & $\begin{array}{l}>\mathrm{CN} \\
>\mathrm{MCI} A-\mathrm{T}- \\
>\mathrm{MCI} \mathrm{A}+\mathrm{T}- \\
>\mathrm{MCI} \mathrm{A}+\mathrm{T}+\end{array}$ \\
\hline
\end{tabular}

Results are means (SD) or numbers (\%). ${ }^{\mathrm{A}} \mathrm{P}<0.05$ compared to MCI A-T+Cluster $\mathrm{A} ;{ }^{\mathrm{B}} \mathrm{P}<0.05$ compared to MCI $\mathrm{A}-\mathrm{T}+$ Cluster $\mathrm{B} ;{ }^{\mathrm{C}} \mathrm{P}<0.05$ compared to $\mathrm{MCI} \mathrm{A}-\mathrm{T}+$ Cluster $\mathrm{C}$; Additional comparisons to other biomarker groups were set at $\mathrm{p}<0.05$. CSF values were log transformed to test statistical differences between groups. \#Center measured values using ELISA; some EDAR cases were not included here as another CSF assay was used. The A $\beta 1$ 42/A $\beta 1-40$ ratio was multiplied by 10 . $A \beta=$ beta amyloid; $\mathrm{APOE}=$ apolipoprotein $\mathrm{E} ; \mathrm{MCI}=$ mild cognitive impairment; NFL=neurofilament-light, $\mathrm{Ng}=$ neurogranin; $\mathrm{T}$-tau=total tau; P-tau=phosphorylated tau. 
Table 3: Overview of CSF values for MCI-SNAP clusters and other MCI groups compared to CN A-T-group

\begin{tabular}{|c|c|c|c|c|c|c|}
\hline & $\begin{array}{l}\text { MCI } \\
\text { A-T- }\end{array}$ & $\begin{array}{l}\mathrm{MCI} \\
\mathrm{A}+\mathrm{T}-\end{array}$ & $\begin{array}{l}\text { MCI } \\
\text { SNAP } \\
\text { Cluster A }\end{array}$ & $\begin{array}{l}\text { MCI } \\
\text { SNAP } \\
\text { Cluster B }\end{array}$ & $\begin{array}{l}\text { MCI } \\
\text { SNAP } \\
\text { Cluster C }\end{array}$ & $\begin{array}{l}\mathrm{MCI} \\
\mathrm{A}+\mathrm{T}+\end{array}$ \\
\hline$A \beta 1-38$ & 0 & -- & ++ & + & ++ & 0 \\
\hline$A \beta 1-40$ & 0 & - & ++ & + & ++ & 0 \\
\hline$A \beta 1-42$ & 0 & -- & ++ & 0 & 0 & -- \\
\hline$A \beta 1-42 / A \beta 1-40$ ratio & 0 & - & 0 & - & - & -- \\
\hline NFL & 0 & 0 & ++ & $++^{*}$ & ++ & ++ \\
\hline $\mathrm{Ng}$ & 0 & 0 & ++ & + & +++ & ++ \\
\hline YKL-40 & 0 & 0 & +++ & ++ & +++ & ++ \\
\hline T-tau & 0 & 0 & ++ & ++ & +++ & ++ \\
\hline P-tau & 0 & 0 & ++ & + & +++ & ++ \\
\hline$A P O E-\varepsilon 4$ carriership & $29 \%$ & $40 \%$ & $19 \%$ & $42 \%$ & $73 \%$ & $64 \%$ \\
\hline
\end{tabular}

' 0 ' indicates the absence of pathology, and refers to the CN A-T- reference group; ' + ' indicates elevated levels compared to CN A-T-; '-' indicates decreased levels compared to CN A-T-. A $\beta=$ beta amyloid; " after correction for demographics NFL levels were not statistically different from CN A-T-anymore; APOE=apolipoprotein E; $\mathrm{MCI}=$ mild cognitive impairment; NFL=neurofilament-light, $\mathrm{Ng}=$ neurogranin; $\mathrm{T}$-tau=total tau; P-tau=phosphorylated tau.

\section{Biomarker correlations in MCI-SNAP}

Supplementary Table 3 shows correlations between CSF markers in the total MCI group and in the MCI-SNAP group. In the total MCI group, we found overall moderate to high correlations between amyloid markers $A \beta 38, A \beta 40, A \beta 42, A \beta 42 / A \beta 40$ ratio (rho=0.10.9 ), except the $A \beta 42 / A \beta 40$ ratio was not associated with $A \beta 38$ levels. We also found moderate to high correlations between markers of neuronal injury, synaptic function, and astrocyte activation (t-tau, p-tau, NFL, Ng, YKL-40; rho=0.4-0.7). Amyloid markers correlated well with neuronal injury markers ( $\mathrm{rho}=-0.1-0.6)$, except that $\mathrm{A} \beta 42$ was not associated with NFL, Ng, and YKL-40 levels. Correlations between CSF markers in the MCISNAP group were generally similar to those in the total MCI group, except that the relation between amyloid and neuronal injury markers was somewhat less strong in the MCISNAP group.

\section{Post hoc analyses}

When using only p-tau as measure of tau pathophysiology for subject classifications, somewhat fewer people were identified with tau pathophysiology (50\% vs. 59\%) but we found similar biomarker results regarding MCI-SNAP and the cluster analyses revealed three similar subgroups (data not shown). 


\section{DISCUSSION}

MCI-SNAP is characterized by strongly increased levels of non-aggregation-prone forms of CSF $A \beta$ (i.e. A $\beta 38$ and $A \beta 40$ ), while the concentrations of markers of axonal loss, synaptic function, and astrocyte activation are also increased. Moreover, MCI-SNAP was found to be an etiologically heterogeneous condition, involving several subgroups including a small subgroup with an abnormal $A \beta 42 / A \beta 40$ ratio suggestive of $A D$.

The increase in non-aggregation-prone forms of $A \beta$ was striking and differentiated MCISNAP from the other biomarker-based MCI groups. Given the heterogeneity in the MCISNAP group, the underlying causes of this increase may differ between SNAP subgroups. One possible explanation for the increase in all $A \beta$ isoforms is an increased synaptic activity as this is associated with $A \beta$ release. ${ }^{23}$ Still, the cause of this increased synaptic activity remains to be established. ${ }^{23}$ The increase in $A \beta$ isoforms could also be a compensatory response to brain pathology $y^{24}$ or could reflect a relatively benign condition in which the increased $A \beta$ production is genetically based. However, a benign condition seems unlikely as the MCI-SNAP group had tau pathology, axonal loss and synaptic and astrocytic dysfunction as well. Still, increased $A \beta$ production could be genetically based. Lastly, it is possible that high levels of $A \beta$ isoforms reflect early $A D$ as some animal studies and studies on autosomal dominant $\mathrm{AD}$ showed that $\mathrm{A} \beta 42$ and $\mathrm{A} \beta 40$ levels increase just before or around the time of initial $\mathrm{A} \beta$ plaques formation. ${ }^{25,26}$

Our cluster analyses revealed that MCI-SNAP is not one homogeneous group but rather consists of biologically defined subgroups. Cluster A included individuals with increased levels of all measured CSF markers. The normal A $\beta 42 / \mathrm{A} \beta 40$ ratio and low APOE $\varepsilon 4$ frequency make it unlikely that this cluster represents $A D$. This subgroup resembles a recently reported subgroup in cognitively normal individuals with both very high CSF A 342 and tau levels who showed increased cognitive decline at follow-up. ${ }^{27}$ The underlying pathophysiology, however, remains to be established. Cluster B included individuals with increased $\mathrm{A} \beta$ peptides but to a lesser extent compared to the other MCI-SNAP subgroups. T-tau and p-tau levels were abnormal, but were also less increased compared to the other SNAP subgroups. It is possible that at least a subset of Cluster B may have AD as the $\mathrm{A} \beta 42 / \mathrm{A} \beta 40$ ratio was decreased compared to A-T- groups. Cluster C likely represents AD given that the ratio of CSF $A \beta 42 / A \beta 40$ was similar to $\mathrm{MCI} A+\mathrm{T}+$ and tau levels were higher compared to all other groups. Although CSF A $\beta 42$ levels were lower compared to the other SNAP Clusters, they were similar to those of the $\mathrm{CN}$ reference group (A-T-). Conceivably, a decrease in $A \beta 42$ levels was masked by the overall increase in $A \beta$ production such that it did not lead to abnormal levels. ${ }^{6,28}$ 
Although CSF NFL, Ng, and YKL-40 are suggested to reflect different pathophysiological processes, they were all strongly associated to each other as well as to tau markers in individuals with MCI-SNAP and other individuals with MCI. CSF NFL is believed to reflect axonal degeneration and is increased in AD but also in other neurodegenerative diseases. $^{7,29}$ The increased NFL levels in all MCI-SNAP groups provide additional evidence for the fact that high NFL levels are not specific to A $\beta$ pathology. ${ }^{30} \mathrm{CSF} \mathrm{Ng}$ is a post-synaptic protein that may reflect $\mathrm{AD}$-specific synaptic dysfunction. ${ }^{8,21,30,31}$ This is partly supported by the fact that MCI-SNAP cluster C, the subgroup with the AD-like profile, had the highest $\mathrm{Ng}$ levels compared to the other groups. However, $\mathrm{Ng}$ levels were also increased in the MCI-SNAP subgroups suggesting that an increase may not be specific to $\mathrm{AD}$ pathology. In contrast, the MCI A+T-group showed (a trend towards) decreased Ng levels relative to A-T- groups. Together this suggests a close relationship of $\mathrm{Ng}$ with $\mathrm{A} \beta$ metabolism. CSF YKL-40 is a measure of astrocyte activation and has shown to be associated with $\mathrm{AD}$ and other neurodegenerative diseases. ${ }^{9,22,31-34}$ The association between increased YKL-40 levels and increased A $\beta 40$ and A $\beta 38$ levels in MCI-SNAP suggests a role of astrocytes in $\mathrm{A} \beta$ clearance and production. ${ }^{35}$

We included two MCI groups with normal CSF tau as a reference in our study. The MCI $\mathrm{A}+\mathrm{N}$ - group had an opposite biomarker profile compared to MCI-SNAP, with decreased levels of $A \beta 38$ and $A \beta 40$ relative to $A-T$ - groups, suggesting a general decreased $A \beta$ production. Still, the $A \beta 42 / A \beta 40$ ratio was also decreased, supporting the presence of underlying AD pathology in this group. The MCI A-T-group had very similar CSF biomarker levels as the CN A-T-group. This suggests that cognitive impairment in these participants is due to processes other than amyloid pathophysiology, tau pathophysiology, axonal loss, synaptic dysfunction, and astrocyte activation.

In the current study, we defined MCI-SNAP based on CSF measures and we may have found different results when MCI-SNAP was based on imaging measures. We previously showed that the concordance between the neuronal injury markers CSF tau and hippocampal volume is relatively low in early $\mathrm{AD}$, which could therefore also result in different MCI-SNAP classifications. ${ }^{36}$ Moreover, studies using amyloid imaging to visualize amyloid plaques could reveal other mechanisms underlying MCI-SNAP. Hence, there is a need to also study pathophysiology in MCI-SNAP defined by imaging markers of A $\beta$ pathology and neuronal injury.

Some limitations of our study should be mentioned. SNAP is an arbitrary biomarkerbased classification based on cut-offs. As a result, some individuals might be close to the A $\beta 42$ cut-off of abnormality although previous studies by both our group and others have suggested that SNAP is not merely a group of misclassified individuals. ${ }^{2,37}$ Furthermore, CSF tau levels were not centrally measured, although previous studies have shown relatively consistent levels across batches for tau markers ${ }^{38}$ and we also used study-specific 
cut-offs and performed analyses with correction for study. The main strengths of the study include the uniquely large sample size and central analysis of CSF A $\beta$ isoforms and emerging CSF measures to address the underlying pathophysiology of MCI-SNAP.

In sum, our results demonstrate that MCI-SNAP is characterized by increased levels of CSF $A \beta 38$ and $A \beta 40$ and increased CSF concentrations of biomarkers reflecting axonal or synaptic loss and astrocyte activation. In clinical practice it would be recommended to use the CSF $A \beta 42 / A \beta 40$ ratio to account for overall $A \beta$ metabolism, which may help to identify individuals with AD who are now being labeled as MCI-SNAP. Our results are the first step towards a positive definition of non-amyloid pathology in MCI-SNAP. Future research is needed to further characterize the pathophysiology of MCI-SNAP, the role of astrocytic activation and synaptic dysfunction in relation to A $\beta$ metabolism, and longitudinal outcome of SNAP subgroups in order to provide a refined diagnosis and prognosis to these individuals. 


\section{REFERENCES}

1. Jack CR, Jr., Knopman DS, Chetelat G, et al. Suspected non-Alzheimer disease pathophysiology--concept and controversy. Nat Rev Neurol. 2016;12(2):117-124.

2. Vos SJ, Verhey F, Frolich L, et al. Prevalence and prognosis of Alzheimer's disease at the mild cognitive impairment stage. Brain : a journal of neurology. 2015;138(Pt 5):1327-1338.

3. Petersen RC, Aisen P, Boeve BF, et al. Mild cognitive impairment due to Alzheimer disease in the community. Annals of neurology. 2013;74(2):199-208.

4. Caroli A, Prestia A, Galluzzi S, et al. Mild cognitive impairment with suspected nonamyloid pathology (SNAP): Prediction of progression. Neurology. 2015;84(5):508-515.

5. Schreiber S, Schreiber F, Lockhart SN, et al. Alzheimer Disease Signature Neurodegeneration and APOE Genotype in Mild Cognitive Impairment With Suspected Non-Alzheimer Disease Pathophysiology. JAMA neurology. 2017;74(6):650-659.

6. Spies PE, Slats D, Sjogren JM, et al. The cerebrospinal fluid amyloid beta42/40 ratio in the differentiation of Alzheimer's disease from non-Alzheimer's dementia. Curr Alzheimer Res. 2010;7(5):470-476.

7. Sjogren M, Blomberg M, Jonsson M, et al. Neurofilament protein in cerebrospinal fluid: a marker of white matter changes. J Neurosci Res. 2001;66(3):510-516.

8. Thorsell A, Bjerke M, Gobom J, et al. Neurogranin in cerebrospinal fluid as a marker of synaptic degeneration in Alzheimer's disease. Brain research. 2010;1362:13-22.

9. Querol-Vilaseca M, Colom-Cadena M, Pegueroles J, et al. YKL-40 (Chitinase 3-like I) is expressed in a subset of astrocytes in Alzheimer's disease and other tauopathies. J Neuroinflammation. 2017;14(1):118.

10. Bos I, Vos, S.J.B., Vandenberghe, R., Scheltens, P., Engelborghs, S., Frisoni, G., Molinuevo, J., Wallin, A., Lléo, A., Popp, J., Martinez-Lage, P., Baird, A., Dobson, R., Legido-Quigley, C., Bertram, L., Sleegers, K., Kate ten, M., Barkhof, F., Zetterberg, H., Lovestone, S., Streffer, J., Visser, P. . The EMIF-AD Multimodal Biomarker Discovery Study: Design, methods and cohort characteristics. Alzheimer's research \& therapy. 2018.

11. van der Flier WM, Pijnenburg YA, Prins N, et al. Optimizing patient care and research: the Amsterdam Dementia Cohort. Journal of Alzheimer's disease : JAD. 2014;41(1):313-327.

12. Somers C, Struyfs H, Goossens J, et al. A Decade of Cerebrospinal Fluid Biomarkers for Alzheimer's Disease in Belgium. Journal of Alzheimer's disease : JAD. 2016;54(1):383-395.

13. Estanga A, Ecay-Torres M, Ibanez A, et al. Beneficial effect of bilingualism on Alzheimer's disease CSF biomarkers and cognition. Neurobiology of aging. 2017;50:144-151.

14. Tautvydaite D, Kukreja D, Antonietti JP, Henry H, von Gunten A, Popp J. Interaction between personality traits and cerebrospinal fluid biomarkers of Alzheimer's disease pathology modulates cognitive performance. Alzheimer's research \& therapy. 2017;9(1):6.

15. Bos I, Verhey FR, Ramakers I, et al. Cerebrovascular and amyloid pathology in predementia stages: the relationship with neurodegeneration and cognitive decline. Alzheimer's research \& therapy. 2017;9(1):101.

16. Visser PJ, Verhey FR, Boada M, et al. Development of screening guidelines and clinical criteria for predementia Alzheimer's disease. The DESCRIPA Study. Neuroepidemiology. 2008;30(4):254-265.

17. Reijs BLR, Ramakers I, Elias-Sonnenschein L, et al. Relation of Odor Identification with Alzheimer's Disease Markers in Cerebrospinal Fluid and Cognition. Journal of Alzheimer's disease : JAD. 2017;60(3):1025-1034.

18. Galluzzi S, Marizzoni M, Babiloni C, et al. Clinical and biomarker profiling of prodromal Alzheimer's disease in workpackage 5 of the Innovative Medicines Initiative PharmaCog project: a 'European ADNI study'. J Intern Med. 2016;279(6):576-591.

19. Petersen RC. Mild cognitive impairment as a diagnostic entity. Journal of internal medicine. 2004;256(3):183-194.

20. Zetterberg H, Skillback T, Mattsson N, et al. Association of Cerebrospinal Fluid Neurofilament Light Concentration With Alzheimer Disease Progression. JAMA neurology. 2016;73(1):60-67. 
21. Portelius E, Zetterberg H, Skillback T, et al. Cerebrospinal fluid neurogranin: relation to cognition and neurodegeneration in Alzheimer's disease. Brain : a journal of neurology. 2015;138(Pt 11):3373-3385.

22. Olsson B, Hertze J, Lautner R, et al. Microglial markers are elevated in the prodromal phase of Alzheimer's disease and vascular dementia. Journal of Alzheimer's disease : JAD. 2013;33(1):45-53.

23. Cirrito JR, Yamada KA, Finn MB, et al. Synaptic activity regulates interstitial fluid amyloid-beta levels in vivo. Neuron. 2005;48(6):913-922.

24. Stargardt A, Swaab DF, Bossers K. Storm before the quiet: neuronal hyperactivity and Abeta in the presymptomatic stages of Alzheimer's disease. Neurobiology of aging. 2015;36(1):1-11.

25. Maia LF, Kaeser SA, Reichwald J, et al. Increased CSF Abeta during the very early phase of cerebral Abeta deposition in mouse models. EMBO Mol Med. 2015;7(7):895-903.

26. Bateman RJ, Xiong C, Benzinger TL, et al. Clinical and biomarker changes in dominantly inherited Alzheimer's disease. N Engl J Med. 2012;367(9):795-804.

27. de Leon MJ, Pirraglia E, Osorio RS, et al. The nonlinear relationship between cerebrospinal fluid Abeta 42 and tau in preclinical Alzheimer's disease. PloS one. 2018;13(2):e0191240.

28. Wiltfang J, Esselmann H, Bibl M, et al. Amyloid beta peptide ratio $42 / 40$ but not A beta 42 correlates with phospho-Tau in patients with low- and high-CSF A beta 40 load. J Neurochem. 2007;101(4):1053-1059.

29. Skillback T, Farahmand B, Bartlett JW, et al. CSF neurofilament light differs in neurodegenerative diseases and predicts severity and survival. Neurology. 2014;83(21):1945-1953.

30. Mattsson N, Insel PS, Palmqvist S, et al. Cerebrospinal fluid tau, neurogranin, and neurofilament light in Alzheimer's disease. EMBO Mol Med. 2016;8(10):1184-1196.

31. Janelidze S, Hertze J, Zetterberg H, et al. Cerebrospinal fluid neurogranin and YKL-40 as biomarkers of Alzheimer's disease. Ann Clin Transl Neurol. 2016;3(1):12-20.

32. Craig-Schapiro R, Perrin RJ, Roe CM, et al. YKL-40: a novel prognostic fluid biomarker for preclinical Alzheimer's disease. Biological psychiatry. 2010;68(10):903-912.

33. Antonell A, Mansilla A, Rami L, et al. Cerebrospinal fluid level of YKL-40 protein in preclinical and prodromal Alzheimer's disease. Journal of Alzheimer's disease : JAD. 2014;42(3):901-908.

34. Alcolea D, Carmona-Iragui M, Suarez-Calvet M, et al. Relationship between beta-Secretase, inflammation and core cerebrospinal fluid biomarkers for Alzheimer's disease. Journal of Alzheimer's disease : JAD. 2014;42(1):157-167.

35. Frost GR, Li YM. The role of astrocytes in amyloid production and Alzheimer's disease. Open Biol. 2017;7(12).

36. Vos SJB, Gordon BA, Su Y, et al. NIA-AA staging of preclinical Alzheimer disease: discordance and concordance of CSF and imaging biomarkers. Neurobiology of aging. 2016;44:1-8.

37. Mormino EC, Betensky RA, Hedden T, et al. Synergistic effect of beta-amyloid and neurodegeneration on cognitive decline in clinically normal individuals. JAMA neurology. 2014;71(11):1379-1385.

38. Schindler SE, Sutphen CL, Teunissen C, et al. Upward drift in cerebrospinal fluid amyloid beta 42 assay values for more than 10 years. Alzheimer's \& dementia : the journal of the Alzheimer's Association. 2018;14(1):62-70. 


\section{SUPPLEMENTAL DATA}

Supplemental Table 1: Center specific cut-offs to define tau abnormality

\begin{tabular}{llll}
\hline Study & $\mathrm{n}$ & $\begin{array}{l}\text { CSF t-tau } \\
\text { cut-off }\end{array}$ & $\begin{array}{l}\text { CSF p-tau } \\
\text { cut-off }\end{array}$ \\
\hline Amsterdam & 92 & $>375$ & $>52$ \\
Antwerp & 102 & $>296.5$ & $>56.5$ \\
DESCRIPA $^{*}$ & 19 & $>375$ & $>52$ \\
EDAR $^{*}$ & 70 & $>98$ Luminex & $>35$ Luminex \\
GAP & 37 & $>375$ ELISA & $>52$ ELISA \\
Lausanne & 17 & $>350$ & $>61$ \\
Maastricht & 32 & $>286$ & $>55$ \\
Pharmacog & 69 & $>350$ & $>85$ \\
\hline
\end{tabular}

CSF analyses were performed using the Innotest ELISA (Fujirebio Europe) for all studies expect for a subset of EDAR, which used the multiplex xMAP Luminex Platform. ${ }^{\star}$ For multicenter studies, biomarker analyses were performed centrally. T-tau=total tau; $\mathrm{p}$-tau=phosphorylated tau. 
Chapter 9

Supplemental Table 2. CSF values by A $\beta 1-42 / A \beta 1-40$ MCI-SNAP subgroup

\begin{tabular}{|c|c|c|c|c|}
\hline & $\begin{array}{l}\text { MCI A-T+ } \\
\text { A } \beta 1-42 / 40 \text { low } \\
\mathrm{N}=32\end{array}$ & $\begin{array}{l}\text { MCI A-T+ } \\
\text { A } \beta 1-42 / 40 \text { high } \\
N=55\end{array}$ & $\begin{array}{l}\text { A } \beta 1-42 / 40 \text { low: } \\
\text { comparison to } \\
\text { other groups }\end{array}$ & $\begin{array}{l}\text { A } \beta 1-42 / 40 \text { high: } \\
\text { comparison to } \\
\text { other groups }\end{array}$ \\
\hline Age & $\begin{array}{l}70.5 \\
(6.7)\end{array}$ & $\begin{array}{l}71.7 \\
(7.3)\end{array}$ & $\begin{array}{l}>\mathrm{CN} \\
>\mathrm{MCI} \text { A-T- }\end{array}$ & $\begin{array}{l}>\mathrm{CN} \\
>\mathrm{MCI} \text { A-T- }\end{array}$ \\
\hline Female, $n$ & $\begin{array}{l}21 \\
(66)\end{array}$ & $\begin{array}{l}32 \\
(58)\end{array}$ & - & - \\
\hline Education, $y$ & $\begin{array}{l}11.3 \\
(3.6)\end{array}$ & $\begin{array}{l}10.5 \\
(4.1)\end{array}$ & - & - \\
\hline$A P O E-\varepsilon 4, n$ & $\begin{array}{l}25^{\mathrm{H}} \\
(81) \\
\varepsilon 4 \varepsilon 423 \% \\
\varepsilon 4 \varepsilon 358 \% \\
\varepsilon 3 \varepsilon 319 \%\end{array}$ & $\begin{array}{l}9 \\
(17) \\
\varepsilon 4 \varepsilon 317 \% \\
\varepsilon 3 \varepsilon 364 \% \\
\varepsilon 2 \varepsilon 319 \%\end{array}$ & $\begin{array}{l}>\mathrm{CN} \\
>\mathrm{MCI} \mathrm{A}-\mathrm{T}- \\
>\mathrm{MCI} \mathrm{A}+\mathrm{T}-\end{array}$ & $\begin{array}{l}<\mathrm{MCI} A+\mathrm{T}- \\
<\mathrm{MCI} \mathrm{A}+\mathrm{T}+\end{array}$ \\
\hline MMSE & $\begin{array}{l}26.6 \\
(2.6)\end{array}$ & $\begin{array}{l}27.1 \\
(2.1)\end{array}$ & $<\mathrm{CN}$ & $\begin{array}{l}<\mathrm{CN} \\
>\mathrm{MCI} \mathrm{A}+\mathrm{T}+\end{array}$ \\
\hline NFL, pg/mL & $\begin{array}{l}1185 \\
(492)\end{array}$ & $\begin{array}{l}1417 \\
(926)\end{array}$ & $\begin{array}{l}>\mathrm{CN} \\
>\mathrm{MCI} A-\mathrm{T}- \\
>\mathrm{MCI} \mathrm{A}+\mathrm{T}-\end{array}$ & $\begin{array}{l}>\mathrm{CN} \\
>\mathrm{MCI} A-\mathrm{T}- \\
>\mathrm{MCI} \mathrm{A}+\mathrm{T}-\end{array}$ \\
\hline $\mathrm{Ng}, \mathrm{pg} / \mathrm{mL}$ & $\begin{array}{l}251 \\
(141)\end{array}$ & $\begin{array}{l}160 \\
(95)\end{array}$ & $\begin{array}{l}>\mathrm{CN} \\
>\text { MCI A-T- } \\
>\text { MCI A+T- } \\
>\text { MCI A+T+ }\end{array}$ & $\begin{array}{l}>\mathrm{CN} \\
>\mathrm{MCI} A-\mathrm{T}- \\
>\mathrm{MCI} \mathrm{A}+\mathrm{T}-\end{array}$ \\
\hline YKL-40, ng/mL & $\begin{array}{l}220 \\
(78)\end{array}$ & $\begin{array}{l}217 \\
(96)\end{array}$ & $\begin{array}{l}>\mathrm{CN} \\
>\mathrm{MCI} A-\mathrm{T}- \\
>\mathrm{MCI} \mathrm{A}+\mathrm{T}-\end{array}$ & $\begin{array}{l}>\mathrm{CN} \\
>\mathrm{MCI} A-\mathrm{T}- \\
>\mathrm{MCI} \mathrm{A}+\mathrm{T}-\end{array}$ \\
\hline $\mathrm{A} \beta 1-38, \mathrm{pg} / \mathrm{mL}$ & $\begin{array}{l}3624 \\
(774)\end{array}$ & $\begin{array}{l}3191 \\
(898)\end{array}$ & $\begin{array}{l}>\mathrm{CN} \\
>\text { MCI A-T- } \\
>\text { MCI A+T- } \\
>\text { MCI A+T+ }\end{array}$ & $\begin{array}{l}>\mathrm{CN} \\
>\text { MCI A-T- } \\
>\text { MCI A+T- } \\
>\text { MCI A+T+ }\end{array}$ \\
\hline $\mathrm{A} \beta 1-40, \mathrm{pg} / \mathrm{mL}$ & $\begin{array}{l}8054 \\
(1413)\end{array}$ & $\begin{array}{l}7015 \\
(1826)\end{array}$ & $\begin{array}{l}>\mathrm{CN} \\
>\mathrm{MCI} \text { A-T- } \\
>\text { MCI A+T- } \\
>\text { MCI A+T+ }\end{array}$ & $\begin{array}{l}>\mathrm{CN} \\
>\mathrm{MCI} \text { A-T- } \\
>\text { MCI A+T- } \\
>\text { MCI A+T+ }\end{array}$ \\
\hline $\mathrm{A} \beta 1-42, \mathrm{pg} / \mathrm{mL}$ & $\begin{array}{l}380^{\mathrm{H}} \\
(57)\end{array}$ & $\begin{array}{l}645 \\
(225)\end{array}$ & $\begin{array}{l}<\mathrm{CN} \\
<\mathrm{MCI} \text { A-T- } \\
>\text { MCI A+T- } \\
>\text { MCI A+T+ }\end{array}$ & $\begin{array}{l}>\mathrm{CN} \\
>\mathrm{MCI} \mathrm{A}+\mathrm{T}- \\
>\mathrm{MCI} \mathrm{A}+\mathrm{T}+\end{array}$ \\
\hline $\mathrm{A} \beta 1-42 / \mathrm{A} \beta 1-40$ ratio & $\begin{array}{l}0.48^{\mathrm{H}} \\
(0.07)\end{array}$ & $\begin{array}{l}0.91 \\
(0.14)\end{array}$ & $\begin{array}{l}<\mathrm{CN} \\
<\mathrm{MCI} A-\mathrm{T}- \\
<\mathrm{MCI} A+\mathrm{T}- \\
>\mathrm{MCI} \mathrm{A}+\mathrm{T}+\end{array}$ & $\begin{array}{l}>\text { MCI A+T- } \\
>\text { MCI A+T+ }\end{array}$ \\
\hline T-tau, pg/mL\# & $\begin{array}{l}713^{\mathrm{H}} \\
(216)\end{array}$ & $\begin{array}{l}441 \\
(122)\end{array}$ & $\begin{array}{l}>\mathrm{CN} \\
>\mathrm{MCI} \mathrm{A}-\mathrm{T}- \\
>\mathrm{MCI} \mathrm{A}+\mathrm{T}-\end{array}$ & $\begin{array}{l}>\mathrm{CN} \\
>\text { MCI A-T- } \\
>\text { MCI A+T- } \\
<\text { MCI A+T+ }\end{array}$ \\
\hline P-tau, pg/mL\# & $\begin{array}{l}102^{\mathrm{H}} \\
(34)\end{array}$ & $\begin{array}{l}68 \\
(19)\end{array}$ & $\begin{array}{l}>\mathrm{CN} \\
>\mathrm{MCI} \mathrm{A}-\mathrm{T}- \\
>\mathrm{MCI} \mathrm{A}+\mathrm{T}-\end{array}$ & $\begin{array}{l}>\mathrm{CN} \\
>\text { MCI A-T- } \\
>\text { MCI A+T- } \\
<\text { MCI A+T+ }\end{array}$ \\
\hline
\end{tabular}

Results are means (SD) or numbers (\%). ${ }^{\mathrm{H}} \mathrm{P}<0.05$ compared to MCI A-T+ A $\beta 1-42 / \mathrm{A} \beta 1-40$ high. CSF values were log transformed to test statistical differences between groups. \#Center measured values using ELISA; some EDAR cases were not included here as another CSF assay was used. The A $\beta 1-42 / A \beta 1-40$ ratio was multiplied by 10 . $A=$ amyloid; $A \beta=$ beta amyloid; $A P O E=$ apolipoprotein $\mathrm{E} ; \mathrm{MCI}=$ mild cognitive impairment; $\mathrm{NFL}=$ neurofilament-light, $\mathrm{Ng}=$ neurogranin; $\mathrm{T}=\mathrm{tau} ; \mathrm{T}$-tau=total tau; $\mathrm{P}$-tau=phosphorylated tau. 
Supplemental Table 3. Correlation between CSF markers in the total MCI population (upper right) and MCISNAP group (lower left)

\begin{tabular}{|c|c|c|c|c|c|c|c|c|c|}
\hline & NFL & $\mathrm{Ng}$ & YKL-40 & $A \beta 1-38$ & $\mathrm{~A} \beta 1-40$ & $\mathrm{~A} \beta 1-42$ & $A \beta 1-42 / 40$ & T-tau\# & P-tau\# \\
\hline NFL & 1 & $.347^{\star *}$ & $.545^{\star \star}$ & $.277^{\star \star}$ & $.274^{\star *}$ & .002 & $-.231^{\star \star}$ & $.498^{\star *}$ & $.443^{* *}$ \\
\hline $\mathrm{Ng}$ & .161 & 1 & $.482^{\star *}$ & $.610^{* *}$ & $.537^{\star *}$ & .073 & $-.352^{\star *}$ & $.722^{\star *}$ & $.762^{\star *}$ \\
\hline YKL-40 & $.550^{* *}$ & $.353^{* *}$ & 1 & $.435^{* *}$ & $.362^{* *}$ & .054 & $-.259^{* *}$ & $.581^{\star *}$ & $.573^{* *}$ \\
\hline$A \beta 1-38$ & $.218^{*}$ & $.701^{\star *}$ & $.380^{* *}$ & 1 & $.964^{* *}$ & $.622^{\star *}$ & .064 & $.510^{\star *}$ & $.540^{* *}$ \\
\hline $\mathrm{A} \beta 1-40$ & .198 & $.599^{* *}$ & $.297^{\star *}$ & $.928^{* *}$ & 1 & $.687^{\star *}$ & $.122^{* *}$ & $.442^{* *}$ & $.478^{* *}$ \\
\hline$A \beta 1-42$ & .080 & .022 & .098 & $.304^{\star *}$ & $.330^{* *}$ & 1 & $.769^{\star *}$ & $-.138^{\star *}$ & $-.114^{*}$ \\
\hline $\mathrm{A} \beta 1-42 / 1-40$ & -.016 & $-.283^{\star *}$ & -.106 & -.204 & -.200 & $.790^{\star *}$ & 1 & $-.576^{\star *}$ & $-.570^{\star *}$ \\
\hline T-tau\# & .146 & $.670^{* *}$ & $.338^{\star *}$ & $.550^{* *}$ & $.530^{* *}$ & $-.279^{*}$ & $-.587^{\star *}$ & 1 & $.910^{* *}$ \\
\hline P-tau\# & .062 & $.741^{\star *}$ & $.320^{* *}$ & $.661^{\star *}$ & $.627^{\star *}$ & -.181 & $-.492^{\star *}$ & $.774^{\star *}$ & 1 \\
\hline
\end{tabular}

** Spearman's rho correlations significant at the 0.01 level (2-tailed). ${ }^{*}$ Spearman's rho correlations significant at the 0.05 level (2-tailed). \# Center measured values using ELISA; Some EDAR cases were not included here as another CSF assay was used. 


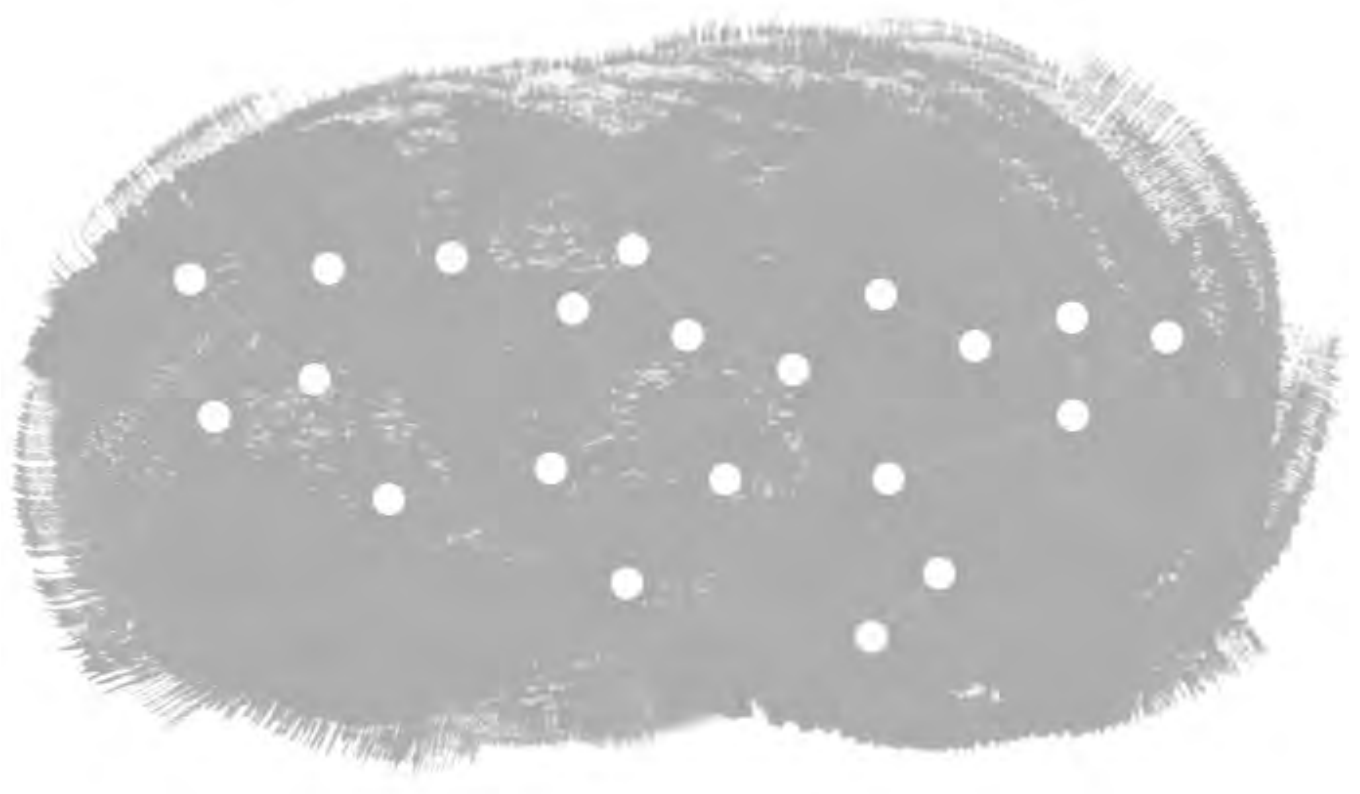



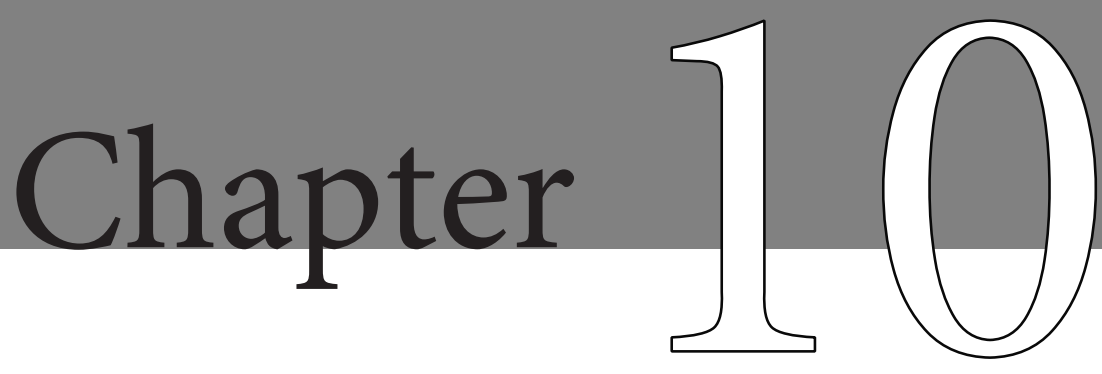

General Discussion 


\section{GENERAL DISCUSSION}

Biomarkers have improved our understanding of the pathophysiology of Alzheimer's disease $(\mathrm{AD}) .{ }^{1-3}$ While aggregation of $\mathrm{A} \beta$-beta $(\mathrm{A} \beta)$ plays a central role in the disease, there are additional mechanisms involved which also need to be understood as they may be potential targets for treatment. The aim of this thesis was to examine how vascular and neurodegenerative mechanisms relate to $A \beta$ aggregation and cognitive decline in the predementia stages of AD. Part I describes the European Medical Information Framework for $\mathrm{AD}$ (EMIF-AD), which served as the methodological basis for this thesis. Part II concentrates on the relationships between vascular factors, AD biomarkers and cognition. Part III centers around established and novel cerebrospinal fluid (CSF) biomarkers and their relation to cognition. In this final chapter, the results from all thesis chapters are integrated and possible implications for research, prevention strategies and clinical practice are considered. Figure 1 shows the investigated associations integrated into a theoretical framework.

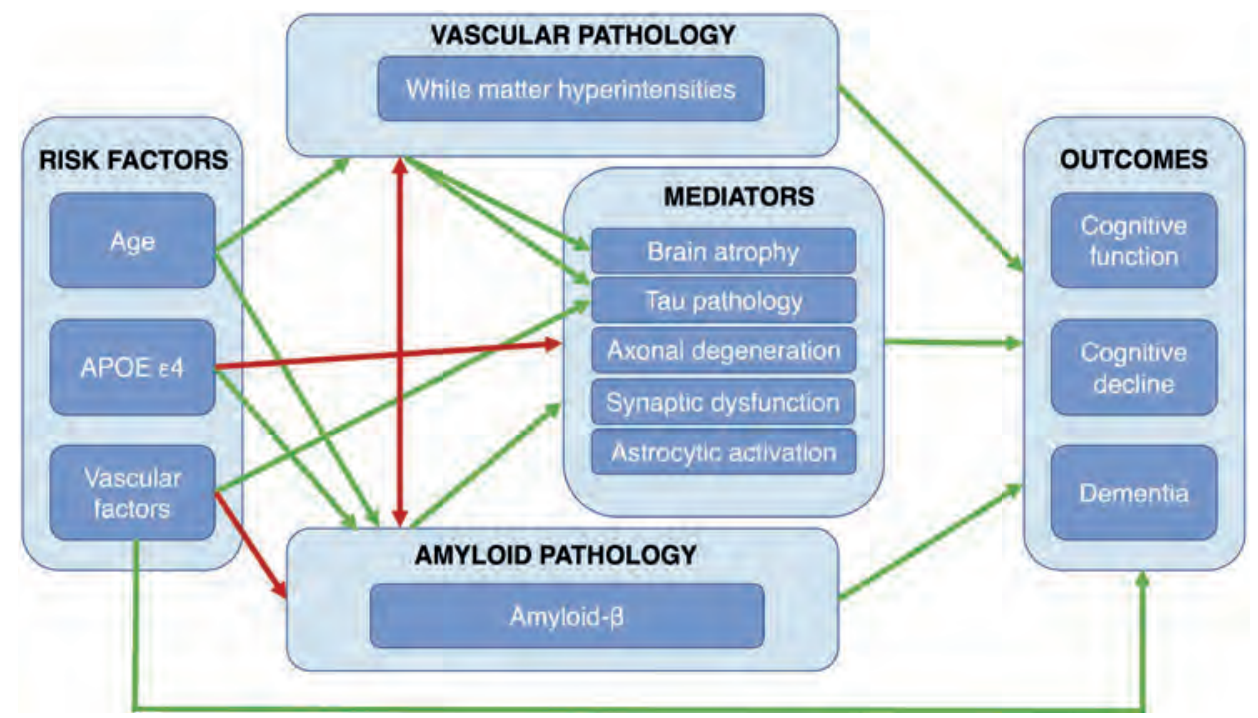

Figure 1. Conceptual framework of the pathophysiology of $\mathrm{AD}$

Green arrows represent statistically significant associations between factors and red arrows represent associations that were not statically significant. 


\section{MAIN FINDINGS}

\section{Vascular pathways in $A D$}

To increase insight into possible vascular contributions to pre-dementia $\mathrm{AD}$, we investigated the relations between vascular risk factors and $\mathrm{AD}$ biomarkers in individuals with mild cognitive impairment (MCI, Chapter 4) and normal cognition (Chapter 5). The interrelationship between $\mathrm{AD}$ biomarkers and vascular pathology, as defined by white matter hyperintensities (WMH) on magnetic resonance imaging (MRI), was tested in individuals with subjective cognitive decline (SCD) and MCI (Chapter 6).

\section{Vascular factors and AD biomarkers}

Hypertension, a normal-to-low body mass index (BMI) and a higher vascular load (Framingham risk score, FRS) were associated with a faster increase in CSF tau concentrations over time in individuals with normal cognition. When stratifying by baseline $A \beta$ status, the effects of hypertension and the FRS on tau were only found in individuals who already had A $\beta$ pathology (Chapter 5). WMH were associated with increased tau levels and more severe medial temporal lobe atrophy (MTA, Chapter 6). The influence of WMH on MTA was independent of that of $A \beta$ pathology, and when both pathologies co-occurred their effects on MTA were additive (Chapter 6). On the contrary, several late-life risk factors were associated with a lower risk of $\mathrm{AD}$ pathology. Obesity was less common in individuals with abnormal tau and normal cognition (Chapter 5) or MCI (Chapter 4), and hypercholesterolemia occurred less frequent in individuals with prodromal $\mathrm{AD}$ (Chapter 4).

\section{Vasculair factors and cognition}

Only in cognitively normal individuals with $A \beta$ and tau pathology (i.e. preclinical AD), we found that at baseline hypertension and a high FRS were associated with lower cognitive performance, while hypercholesterolemia was associated with a better performance. In addition, a high FRS was associated with a faster rate of cognitive decline in individuals with preclinical $\mathrm{AD}$, while hypercholesterolemia was associated with a slower rate of cognitive decline in the total group with normal cognition (Chapter 5). In prodromal AD, we found that none of the risk factors were associated with an increased rate of cognitive decline, while alcohol use was associated with an increased rate regardless of AD pathology (Chapter 4). In Chapter 6, we found that WMH were associated with cognitive impairment and decline in non-demented memory clinic patients, but having co-occurring $\mathrm{WMH}$ and $\mathrm{A} \beta$ pathology did not further increase the rate of decline.

Overall these results imply that vascular risk factors do not directly increase the risk of $\mathrm{AD}$, but once $\mathrm{A} \beta$ pathology is present they enhance neurodegeneration in cognitively 
normal individuals, which in turn may contribute to cognitive decline. Vascular pathology such as $\mathrm{WMH}$, also contributes to neurodegeneration, irrespective of $\mathrm{A} \beta$ pathology. In addition, our results confirm the notion that in late life hypercholesterolemia, ${ }^{4,5}$ and obesity $^{6,7}$ are no longer risk factors for $\mathrm{AD}$ as they are inversely associated with $\mathrm{AD}$ pathology and cognition. Weight loss in late life could be due to reduced food intake or metabolic changes associated with early $\mathrm{AD},{ }^{8,9}$ while a reduction in total cholesterol levels may be related to synaptic failure in $\mathrm{AD} \cdot{ }^{10,11}$

\section{Established and novel AD biomarkers}

To obtain a better understanding of the temporal pattern of $\mathrm{AD}$ pathophysiology and the inter-individual variation in cognitive trajectories, we examined how established - A $\beta$ and tau - and novel - neurofilament-light (NFL), neurogranin (Ng) and YKL-40 - CSF biomarkers related to cognitive decline and to carriership of the apolipoprotein $\mathrm{E}(A P O E) \varepsilon 4$ allele, the strongest genetic risk factor for $\mathrm{AD}$ in individuals, across the clinical $\mathrm{AD}$ spectrum.

\section{Relations between established AD biomarkers and cognition}

As expected, we found that $A P O E \varepsilon 4$ carriership was associated with $\mathrm{A} \beta$ pathology across the clinical $\mathrm{AD}$ spectrum (Chapter 3 ), and that $\mathrm{A} \beta$ pathology was associated with lower cognitive scores and a faster rate of decline in individuals with normal cognition (Chapters $3 \& 7)$ and MCI (3, $6 \& 8)$. Moreover, we found that excluding individuals with $\mathrm{A} \beta$ pathology from normative data, improved prognostic accuracy of memory tests in predicting progression to dementia in non-demented memory-clinic patients. In individuals with normal cognition, tau pathology was not associated with cognitive performance, after adjusting for the presence of $A \beta$ pathology (Chapter 7). However, tau pathology could predict future cognitive decline in MCI and demented individuals with and without $\mathrm{A} \beta$ pathology (Chapter 5,6 \& 8).

Relations between novel AD biomarkers with established AD biomarkers and cognition Individuals with normal cognition or MCI who had $\mathrm{A} \beta$ pathology showed higher levels of CSF markers of tau pathology, axonal degeneration (NFL), synaptic dysfunction $(\mathrm{Ng})$ and astroglial activation (YKL-40) compared to individuals without $\mathrm{A} \beta$ pathology (Chapter 8). NFL, Ng, and YKL-40 levels were not associated with the APOE $\varepsilon 4$ allele (Chapter 8). When examining the CSF biomarker profiles of individuals with MCI and neuronal injury but without evidence of $A \beta$ pathology, a biomarker profile referred to as Suspected non-Alzheimer's disease Pathophysiology (SNAP), we found that these individuals also had higher levels NFL, Ng and YKL-40, compared to cognitively normal individuals with normal $\mathrm{A} \beta$ and normal tau (Chapter 9). In addition, among individuals with MCI-SNAP we identified three biomarker-based subgroups that differed in the degree of neuronal activity, but all SNAP subgroups had strongly increased levels of non-aggregating forms 
of $\mathrm{A} \beta$ isoforms in CSF, compared to the control group (Chapter 9). With regards to cognition, we found that NFL mirrored tau pathology, as both were associated with lower cognitive performance and a faster rate of decline in the MCI and dementia stages, irrespective of $\mathrm{A} \beta$ status (Chapter 8 ). $\mathrm{Ng}$ was associated with worse memory performance in preclinical AD, whereas YKL-40 was associated with cognitive decline only in individuals with MCI or dementia without $\mathrm{A} \beta$ (Chapter 8 ).

Taken together these results confirm that the $A P O E \varepsilon 4$ allele only increases the risk of $A \beta$ pathology, but not that of generic neurodegenerative processes like tau deposition, axonal degeneration, synaptic dysfunction and astroglial activation. ${ }^{12,13}$ Axonal degeneration, synaptic dysfunction and astroglial activation were all strongly associated with $\mathrm{A} \beta$ pathology, even in cognitively normal individuals. However, these processes were not specific to $\mathrm{A} \beta$ pathology as we also observed increased levels in individuals with MCI but without evidence of $A \beta$ pathology. To understand how these two seemingly contrary findings fit into the overall framework of $\mathrm{AD}$ pathophysiology, we not only stratified the individuals from the EMIF-AD Multimodal Biomarker Discovery study (Chapter 3 ) by A $\beta$ status but also split the group without $\mathrm{A} \beta$ into two groups: with tau $(\mathrm{A} \beta-\mathrm{T}+, \mathrm{SNAP})$ and without tau (A $\beta$ - T-; Figure 2).

Figure 2 suggests that with regards to Ng, YKL-40 and NFL levels, profiles of individuals with SNAP may be more similar to those of $A \beta+$ individuals, than to $A \beta$ - T-individuals. One possible explanation for this is that individuals with SNAP have AD but the decrease in $\mathrm{A} \beta$ levels classic for $\mathrm{AD}$ is masked by an overall increase in $\mathrm{A} \beta$ production or they may develop neuronal injury before $\mathrm{A} \beta$ plaques. ${ }^{14}$ Another possibility may be that SNAP and $\mathrm{AD}$ have different underlying pathophysiology, which remains to be clarified in SNAP, that both lead to synaptic integrity, neurodegeneration and astroglial activation.

\section{METHODOLOGICAL CONSIDERATIONS}

\section{Strengths}

A major strength of the studies described in this thesis was the use of large datasets, mainly as a result of harmonizing and combining existing datasets using the EMIF-AD infrastructure (Chapter 2). These voluminous datasets led to high statistical power and allowed for multivariate analyses. Additionally, the datasets we used contained a wide variety of variables regarding biomarkers, risk factors and cognitive tests permitting studies into various aspects of $\mathrm{AD}$. Another strength is that we had longitudinal cognitive outcomes (Chapters 3-8) and longitudinal CSF biomarkers (Chapter 5) yielding the opportunity to study the temporal ordering of AD related events in detail. 

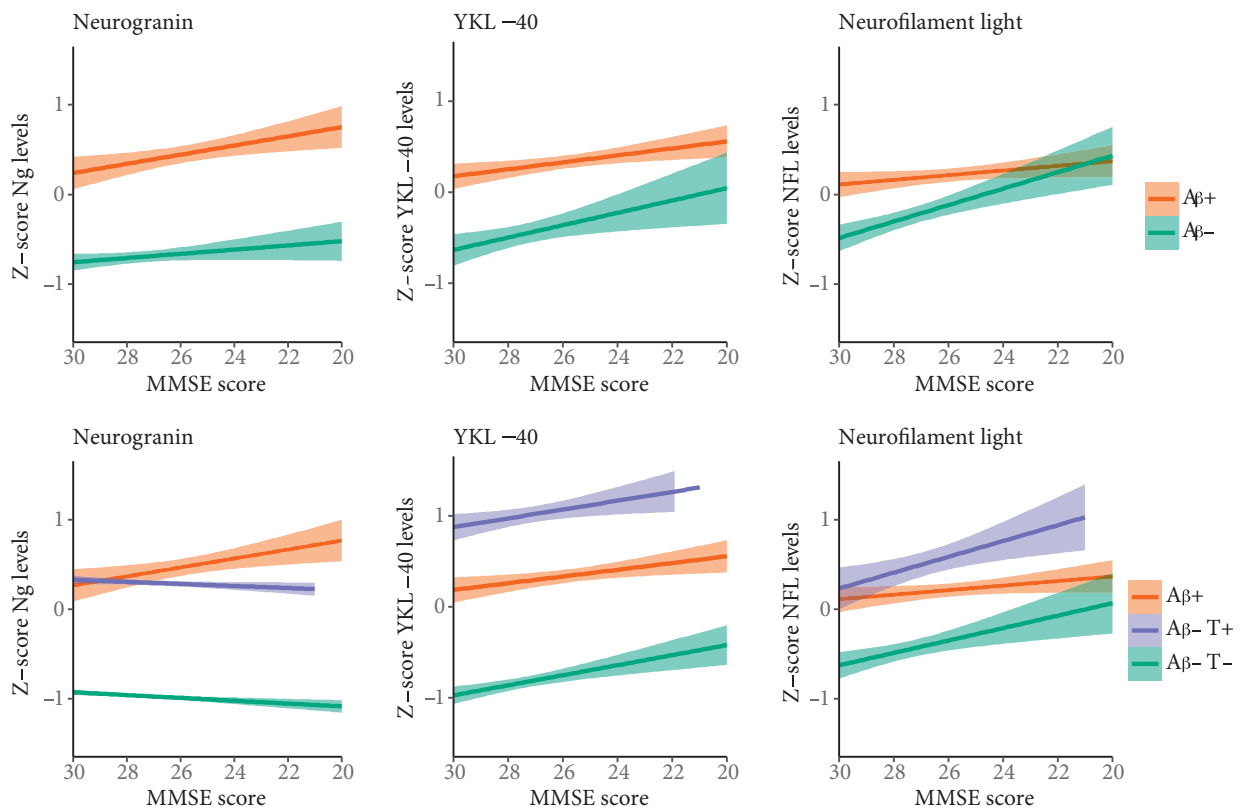

Figure 2: Cross-sectional levels of emerging biomarkers across the cognitive spectrum by A $\beta$ and tau status

Upper panels show standardized neurogranin (left), YKL-40 (middle) and neurofilament-light (right) levels by MMSE scores (lower scores indicated lower cognitive performance) in individuals with $\mathrm{A} \beta$ (A $\beta+$, orange lines) and without $\mathrm{A} \beta$ ( $\mathrm{A} \beta$-, green lines). Lower panels show standardized neurogranin (left), YKL-40 (middle) and neurofilament-light (right) levels by MMSE scores in individuals with $A \beta$ pathology ( $\beta+$, orange lines), without $\mathrm{A} \beta$ but with tau pathology (A $\beta-\mathrm{T}+\mathrm{SNAP}$, purple lines) and without both $\mathrm{A} \beta$ and tau pathology (A $\beta$-T-, green lines).

\section{Limitations}

In most of the conducted studies (all except Chapter 5) we combined data, samples and scans which were collected at different centers using different protocols. This led to preanalytical variability and may have decreased the signal-to-noise ratio. However, the biomarker analyses described in Chapters 3, 8 and 9 were analyzed centrally, and in all studies clinical data was harmonized using standardized values and dichotomous variables. Concerning vascular risk factors (Chapters $4 \& 5$ ), we mostly used indirect measures (e.g. based on medical history or self-report) for the definition, which could cause over- or underreporting of risk factors. The participants we investigated in our studies are unlikely representative of the general population as they were mainly self-selected to participate in research. This, and the exclusion criteria of contributing studies, introduced a selection bias which limits the generalizability of our findings. A clinical diagnosis of AD-type dementia was often used (Chapters 3,4,6-8) instead of a biomarker-based diagnosis, making underlying etiology more heterogeneous. In addition, due to the exploratory nature of our studies we did not always correct for multiple statistical comparisons. 


\section{IMPLICATIONS}

The findings of this thesis provide insight into $\mathrm{AD}$ pathophysiology and may aid to improve diagnostic and prognostic accuracy. This has implications for $\mathrm{AD}$ research, prevention strategies and clinical practice.

\section{Understanding AD pathophysiology}

For the past decades, $\mathrm{AD}$ biomarker research has centered around $\mathrm{A} \beta$ as the primary initiating factor in $\mathrm{AD}$ pathogenesis. Aggregation of $A \beta$ is suggested to trigger downstream consequences such as vascular damage, neurodegeneration, cell death and cognitive decline. ${ }^{2,15,16}$ Although this "amyloid cascade hypothesis" still dominates AD research and is supported by many studies, its comprehensiveness and completeness have also been questioned. ${ }^{17,18}$ Our findings provide directions for the further refinement of the hypothesis in several ways. First, in combination with previous literature ${ }^{19-24}$ our findings suggest that vascular risk factors and pathology do not directly affect $A \beta$. Instead, vascular risk factors enhance neurodegeneration in individuals with $A \beta$ pathology, and vascular pathology $(\mathrm{WMH})$ contributes to neurodegeneration regardless of $\mathrm{A} \beta$ status, and thereby increase the risk of cognitive decline and dementia. These findings support the multifactorial nature of dementia ${ }^{25}$ and suggest several parallel pathways to dementia. Second, we and oth$e^{26,27}$ found that $A \beta$ pathology itself already affects cognitive performance and decline, instead of first triggering downstream neurodegeneration which subsequently affect cognition. Third, our findings indicate that both $A \beta$ and tau are independently associated with processes like astrocytic activation, synaptic dysfunction and axonal degeneration in the pre-dementia stages. Tau-related neuronal injury, synaptic failure and astrocytic activation in turn affect cognition, regardless of the presence of $A \beta$ pathology. Together this suggests that these processes are generic neurodegenerative mechanisms, unspecific to AD. Lastly, our results regarding individuals with SNAP may indicate that some individuals develop neurodegenerative pathology prior to $A \beta$ plaques, although this notion requires validation in future studies using amyloid imaging via positron emission tomography (PET) to visualize $\mathrm{A} \beta$ plaques. Altogether our findings suggest possible heterogeneity in the temporal ordering of pathological events related to $\mathrm{AD}$ or may insinuate a network of parallel pathological AD-related events rather than a strict serial cascade. Future studies are required to further explore these notions and to fully understand the pathophysiology of AD.

\section{AD prevention trials}

Prevention trials for AD can be divided into three categories: primary prevention, aimed to prevent $A \beta$ from accumulating; secondary prevention, aimed to reduce the impact and 
progression of existing pathology prior to the onset of clinical symptoms; and tertiary prevention, aimed to manage manifest disease and maximize the quality of life. Our results may be valuable for primarily secondary prevention trials, in several ways. First, our findings support the notion that $\mathrm{AD}$ is a multifactorial disease, which also calls for a multifactorial secondary prevention. ${ }^{25}$ Besides the ongoing anti-A $\beta$ prevention trials as part of primary $\mathrm{AD}$ prevention, ${ }^{28-30}$ tau immunotherapies, ${ }^{31,32}$ synaptic therapy, ${ }^{33,34}$ and antiinflammatory drugs ${ }^{35}$ should be considered as secondary prevention, possibly simultaneously and directly following anti-A $\beta$ therapy. Even if anti-A $\beta$ therapy would succeed in reducing or stopping $A \beta$ accumulation in the preclinical stage, the downstream consequences of $A \beta$, such as neurodegeneration or synaptic dysfunction, could already be initiated and consequently affect cognition, making treatment ineffective. Therefore, trials could become more beneficial by addressing multiple processes simultaneously at an early stage. Our results also provide implications for lifestyle interventions which generally intent to increase overall mental and physical health and thereby decrease the risk of dementia in the general population. ${ }^{36-39}$ Our findings support early treatment of hypertension as secondary prevention for $\mathrm{AD}$, while reductions in $\mathrm{BMI}$, cholesterol and depression may represent consequences of $\mathrm{AD}$ pathology and may therefore be no straightforward targets for prevention. Moreover, changing multiple aspects of one's lifestyle at once might be too challenging. Hence, lifestyle interventions could become more beneficial by shifting from a one-size-fits-all to personalized approach, accounting for a person's age, socio-economic background and medical history. To accomplish this the relations between all modifiable risk factors and $\mathrm{AD}$ pathology should be investigated thoroughly across several age groups.

\section{Clinical practice}

The findings described in this thesis may also have implications for current clinical practice. We showed that different underlying AD-related mechanisms could contribute to cognitive impairment and decline. Therefore, combining established and novel biomarkers with cognitive tests may help to interpret etiology underlying cognitive impairment and increase prognostic accuracy. However, introducing additional biomarkers into clinical practice also has a downside with the interpretation of complex biomarker profiles. Digital, adaptive guidelines, based on well-established scientific findings, may be helpful to assist clinicians when interpreting biomarker results. Concerning interpretation of neuropsychological results, classifying memory performance into three categories by combining biomarker-adjusted norms with published norms, may improve prognostic accuracy for individual patients and reduce underdiagnosis of $\mathrm{A} \beta$ related cognitive dysfunction. Also in this respect technology could be of assistance as an online tool for the interpretation of cognitive scores using different sets of norms, would be beneficial both 
for clinical practice and research. With regards to vascular risk factors, our findings support the treatment of hypertension, although this would be most beneficial before cognitive problems arise (i.e. in primary care). In addition, clinicians should monitor BMI, cholesterol and depression over time, as a change in one of these factors could be indicative of $\mathrm{AD}$.

\section{Reuse of existing data}

Our findings have demonstrated how reusing and combining existing data can greatly enhance $\mathrm{AD}$ research. By doing this, regular-sized data becomes 'big data' which is a powerful tool to address all kinds of research questions. The EMIF-AD project has been a major player in making $\mathrm{AD}$-related data findable, accessible, interoperable and reusable (i.e. FAIR principles). ${ }^{40}$ The infrastructures developed in the context of EMIF-AD (the Catalogue, the data platform and the AD-Switchbox) have made the realization of research collaborations easier and less time-consuming, and will hopefully be used for future collaborations regarding $\mathrm{AD}$ and could be an inspiration to other disease areas.

\section{FUTURE DIRECTIONS}

Although our studies have provided novel insights and improved our understanding of $\mathrm{AD}$, many questions and uncertainties remain. Through the EMIF-AD facilities large datasets are within grasp, which are of great importance to overcome current challenges in $\mathrm{AD}$ research. One such challenge is to find biomarkers in readily accessible bio-fluids such as blood. ${ }^{41}$ Blood biomarkers for the diagnosis and prognosis of AD would mark an important step forward for future clinical trials, research and clinical practice as they would be inexpensive and minimally invasive to obtain. The EMIF-AD Multimodal Biomarker Discovery study (Chapter 3) aims to identify blood biomarkers by conducting proteomic, metabolomic and genomic analyses, but still other similar studies are required for the validation of possible findings. In addition, longitudinal biomarker studies are of great importance to increase understanding of causal relationships underlying AD. As these studies are expensive, time-consuming and invasive, worldwide collaborations are required to collectively realize such studies. Furthermore, research into the relation between $\mathrm{AD}$ biomarkers and vascular risk factors should also focus on middle-aged individuals, to capture and understand the early mechanisms regarding this relationship. Also, the relation between different aspects of vascular pathology (e.g. microbleeds, lacunar infarcts) and mechanisms like synaptic failure and axonal degeneration needs to examined in more detail, to expand knowledge about the vascular-neurodegeneration connection. To increase insight into the concept of SNAP, studies using amyloid imaging using positron emission tomography (PET) are required to visualize $\mathrm{A} \beta$ plaques and thereby confirm 
our findings regarding CSF biomarkers in individuals with SNAP. ${ }^{42}$ Lastly, it is important to bridge the gap between well-established research findings and clinical practice. One way to do this is by developing software for clinicians which facilitates the storage and interpretation of elaborate patient profiles with data on biomarkers, cognitive testing and co-morbid disorders. This will not only help clinicians to integrate research findings into clinical practice and practice personalized medicine, but it will also facilitate the sharing of clinical data in the context of novel research collaborations. 


\section{REFERENCES}

1. Zetterberg H. Applying fluid biomarkers to Alzheimer's disease. Am J Physiol Cell Physiol. 2017;313(1):C3C10.

2. Jack CR, Knopman DS, Jagust WJ, et al. Tracking pathophysiological processes in Alzheimer's disease: an updated hypothetical model of dynamic biomarkers. The Lancet Neurology. 2013;12(2):207-216.

3. Blennow K, Hampel H. CSF markers for incipient Alzheimer's disease. The Lancet Neurology. 2003;2(10):605-613.

4. Mielke MM, Zandi PP, Sjogren M, et al. High total cholesterol levels in late life associated with a reduced risk of dementia. Neurology. 2005;64(10):1689-1695.

5. Gelber RP, Launer LJ, White LR. The Honolulu-Asia Aging Study: epidemiologic and neuropathologic research on cognitive impairment. Curr Alzheimer Res. 2012;9(6):664-672.

6. Anstey KJ, Cherbuin N, Budge M, Young J. Body mass index in midlife and late-life as a risk factor for dementia: a meta-analysis of prospective studies. Obes Rev. 2011;12(5):e426-437.

7. Xu WL, Atti AR, Gatz M, Pedersen NL, Johansson B, Fratiglioni L. Midlife overweight and obesity increase late-life dementia risk: a population-based twin study. Neurology. 2011;76(18):1568-1574.

8. Cai H, Cong WN, Ji S, Rothman S, Maudsley S, Martin B. Metabolic dysfunction in Alzheimer's disease and related neurodegenerative disorders. Curr Alzheimer Res. 2012;9(1):5-17.

9. Mahieux F, Couderc R, Fenelon G, Maachi M. [Relationships between weight loss and circulating cytokines in patients with Alzheimer's disease]. Psychologie \& neuropsychiatrie du vieillissement. 2006;4(4):281-286.

10. Petrov AM, Kasimov MR, Zefirov AL. Cholesterol in the Pathogenesis of Alzheimer's, Parkinson's Diseases and Autism: Link to Synaptic Dysfunction. Acta Naturae. 2017;9(1):26-37.

11. Koudinov AR, Koudinova NV. Cholesterol, synaptic function and Alzheimer's disease. Pharmacopsychiatry. 2003;36 Suppl 2:S107-112.

12. Morris JC, Roe CM, Xiong C, et al. APOE predicts amyloid-beta but not tau Alzheimer pathology in cognitively normal aging. Annals of neurology. 2010;67(1):122-131.

13. Farfel JM, Yu L, De Jager PL, Schneider JA, Bennett DA. Association of APOE with tau-tangle pathology with and without beta-amyloid. Neurobiology of aging. 2016;37:19-25.

14. Braak H, Zetterberg H, Del Tredici K, Blennow K. Intraneuronal tau aggregation precedes diffuse plaque deposition, but amyloid-beta changes occur before increases of tau in cerebrospinal fluid. Acta neuropathologica. 2013;126(5):631-641.

15. Hardy JA, Higgins GA. Alzheimer's disease: the amyloid cascade hypothesis. Science. 1992;256(5054):184185.

16. Bateman RJ, Xiong C, Benzinger TL, et al. Clinical and biomarker changes in dominantly inherited Alzheimer's disease. The New England journal of medicine. 2012;367(9):795-804.

17. Musiek ES, Holtzman DM. Three dimensions of the amyloid hypothesis: time, space and 'wingmen'. Nat Neurosci. 2015;18(6):800-806.

18. Herrup K. The case for rejecting the amyloid cascade hypothesis. Nat Neurosci. 2015;18(6):794-799.

19. Vemuri P, Lesnick TG, Przybelski SA, et al. Age, vascular health, and Alzheimer disease biomarkers in an elderly sample. Annals of neurology. 2017;82(5):706-718.

20. Lo RY, Jagust WJ, Alzheimer's Disease Neuroimaging I. Vascular burden and Alzheimer disease pathologic progression. Neurology. 2012;79(13):1349-1355.

21. Vemuri P, Knopman DS. The role of cerebrovascular disease when there is concomitant Alzheimer disease. Biochimica et biophysica acta. 2016;1862(5):952-956.

22. Vemuri P, Knopman DS, Lesnick TG, et al. Evaluation of Amyloid Protective Factors and Alzheimer Disease Neurodegeneration Protective Factors in Elderly Individuals. JAMA Neurol. 2017. 
23. Hesse C, Rosengren L, Andreasen N, et al. Transient increase in total tau but not phospho-tau in human cerebrospinal fluid after acute stroke. Neuroscience letters. 2001;297(3):187-190.

24. Petrovitch H, White LR, Izmirilian G, et al. Midlife blood pressure and neuritic plaques, neurofibrillary tangles, and brain weight at death: the HAAS. Honolulu-Asia aging Study. Neurobiology of aging. 2000;21(1):57-62.

25. Iqbal K, Grundke-Iqbal I. Alzheimer's disease, a multifactorial disorder seeking multitherapies. Alzheimer's \& dementia: the journal of the Alzheimer's Association. 2010;6(5):420-424.

26. Vos SJ, Xiong C, Visser PJ, et al. Preclinical Alzheimer's disease and its outcome: a longitudinal cohort study. The Lancet Neurology. 2013;12(10):957-965.

27. Mormino EC, Betensky RA, Hedden T, et al. Synergistic effect of beta-amyloid and neurodegeneration on cognitive decline in clinically normal individuals. JAMA Neurol. 2014;71(11):1379-1385.

28. Mullard A. Sting of Alzheimer's failures offset by upcoming prevention trials. Nature reviews Drug discovery. 2012;11(9):657-660.

29. Sperling RA, Rentz DM, Johnson KA, et al. The A4 study: stopping AD before symptoms begin? Sci Transl Med. 2014;6(228):228fs213.

30. Christensen DD. Changing the course of Alzheimer's disease: anti-amyloid disease-modifying treatments on the horizon. Prim Care Companion J Clin Psychiatry. 2007;9(1):32-41.

31. Pedersen JT, Sigurdsson EM. Tau immunotherapy for Alzheimer's disease. Trends in molecular medicine. 2015;21(6):394-402.

32. Sigurdsson EM. Tau Immunotherapy. Neurodegener Dis. 2016;16(1-2):34-38.

33. Nistico R, Pignatelli M, Piccinin S, Mercuri NB, Collingridge G. Targeting synaptic dysfunction in Alzheimer's disease therapy. Mol Neurobiol. 2012;46(3):572-587.

34. Teich AF, Nicholls RE, Puzzo D, et al. Synaptic therapy in Alzheimer's disease: a CREB-centric approach. Neurotherapeutics. 2015;12(1):29-41.

35. Shadfar S, Hwang CJ, Lim MS, Choi DY, Hong JT. Involvement of inflammation in Alzheimer's disease pathogenesis and therapeutic potential of anti-inflammatory agents. Arch Pharm Res. 2015;38(12):21062119.

36. Livingston G, Sommerlad A, Orgeta V, et al. Dementia prevention, intervention, and care. Lancet. 2017;390(10113):2673-2734.

37. Polidori MC, Nelles G, Pientka L. Prevention of dementia: focus on lifestyle. Int J Alzheimers Dis. 2010;2010.

38. Rosenberg A, Ngandu T, Rusanen M, et al. Multidomain lifestyle intervention benefits a large elderly population at risk for cognitive decline and dementia regardless of baseline characteristics: The FINGER trial. Alzheimer's \& dementia : the journal of the Alzheimer's Association. 2017.

39. Richard E, Van den Heuvel E, van Charante EPM, et al. Prevention of dementia by intensive vascular care (PreDIVA): a cluster-randomized trial in progress. Alzheimer Disease \& Associated Disorders. 2009;23(3):198-204.

40. Wilkinson MD, Dumontier M, Aalbersberg IJ, et al. The FAIR Guiding Principles for scientific data management and stewardship. Sci Data. 2016;3:160018.

41. O'Bryant SE, Mielke MM, Rissman RA, et al. Blood-based biomarkers in Alzheimer disease: Current state of the science and a novel collaborative paradigm for advancing from discovery to clinic. Alzheimer's \& dementia : the journal of the Alzheimer's Association. 2017;13(1):45-58.

42. Degerman Gunnarsson M, Lindau M, Wall A, et al. Pittsburgh compound-B and Alzheimer's disease biomarkers in CSF, plasma and urine: An exploratory study. Dementia and geriatric cognitive disorders. 2010;29(3):204-212. 



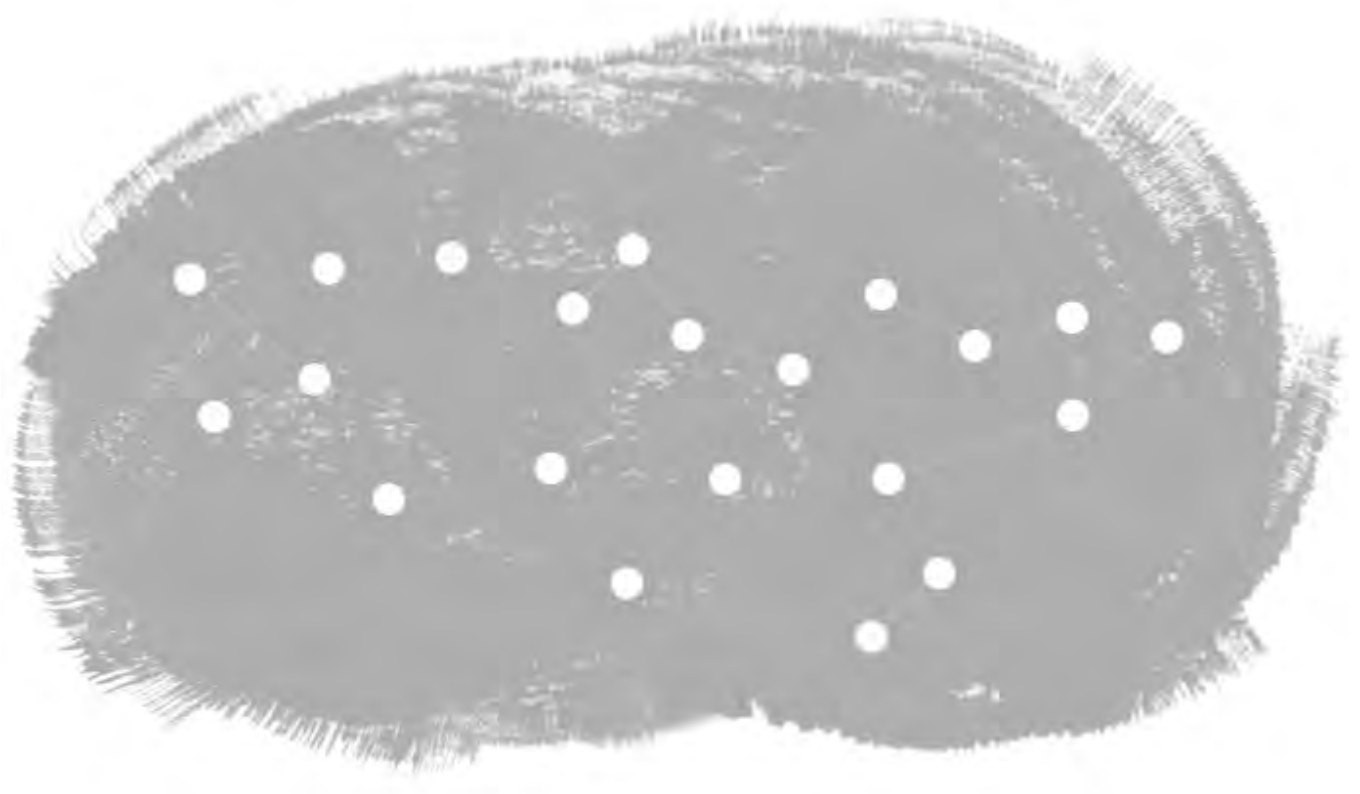




\section{Addendum}

Summary

Nederlandse samenvatting (Dutch summary)

Knowlegde valorization

Publications

Author information

Dankwoord (Acknowlegdements)

Thesis defences from MHeNS 


\section{Summary}

Biomarkers have greatly improved our understanding of the pathophysiology of Alzheimer's disease $(\mathrm{AD})$. However, there are still gaps in current knowledge as biomarker studies thus far have mainly focused on the classical pathological hallmarks of AD: amyloid- $\beta(A \beta)$ plaques and neurofibrillary tangles. In this thesis, we examined how vascular and neurodegenerative mechanisms relate to established $\mathrm{AD}$ biomarkers ( $\mathrm{A} \beta$ and tau) and to cognitive decline in the pre-dementia stages. To investigate this on a large scale we reused and combined existing data in the context of the European Medical Information Framework for AD (EMIF-AD) project, which is described in part I. In part II, we concentrated on the relationship between vascular risk factors and pathology with AD biomarkers. Part III centers around established and novel biomarkers in cerebrospinal fluid (CSF) and how they relate to cognition.

To overcome current challenges in $\mathrm{AD}$ research powerful and generalizable analyses should be conducted, which require large scaled databases. Such voluminous datasets can be realized by reusing and combining existing data. In chapter 2 , we described the EMIFAD infrastructure which served as the methodological backbone of this thesis. The facilities developed in the context of EMIF-AD include an online catalogue with meta-data of cohorts, a data platform for storage of harmonized subject-level data, and the AD-Switchbox which allows for pooled analyses on local databases. Chapter 3 concentrates on one of the major studies of EMIF-AD: the EMIF-AD Multimodal Biomarker Discovery (EMIF-AD MBD) study. In this study data from 1221 individuals originating from 11 cohort studies across Europe were combined. Clinical data was harmonized and plasma, DNA and CSF samples and MRI scans were analyzed centrally. The first analyses on the clinical data confirm the central roles of $\mathrm{A} \beta$ and $A P O E \varepsilon 4$ in $\mathrm{AD}$ pathogenesis. The planned multimodal analyses will provide new insights into underlying mechanisms of $\mathrm{AD}$ and facilitate the discovery of new AD biomarkers.

To increase insight into possible vascular contributions to pre-dementia $\mathrm{AD}$, we investigated the relations between vascular risk factors, vascular pathology, $\mathrm{AD}$ biomarkers and cognition. In chapter 4, we combined data from 14 studies which resulted in a dataset containing 1394 individuals with Mild Cognitive Impairment (MCI). We showed that in individuals with a high likelihood of AD according to their biomarker profile depression, obesity and hypercholesterolemia occurred less frequent compared to individuals with a low likelihood of AD. None of the ten assessed risk factors were associated with a highAD-likelihood biomarker profile. Moreover, we found that only alcohol intake increased the risk of cognitive decline regardless of $\mathrm{AD}$ pathology. Overall these findings suggest that vascular risk factors play a limited role in the prodromal AD stage. Next, as described 
in chapter 5, we tested whether vascular risk factors might play a role in an earlier disease stage, the preclinical stage. In this study, we investigated the relationship between vascular risk factors, $\mathrm{AD}$ biomarkers and cognition in 433 cognitively normal participants from the longitudinal studies at the Knight Alzheimer's Disease Research Center at Washington University in St. Louis. We found that obesity was less common among individuals who had tau pathology and that cardiovascular disorders were more common in individuals with abnormal AD biomarkers, compared to individuals with normal AD biomarkers. Moreover, hypertension, lack of obesity and a high vascular risk score were associated with a faster increase in tau pathology over time, particularly in individuals who already had amyloid pathology. This suggests that treatment of these factors may be considered as secondary prevention strategy for AD. In chapter $\mathbf{6}$, we focused on the interrelationship between $A \beta$ and white matter hyperintensities $(\mathrm{WMH})$, which are a pathological hallmark of cerebrovascular disease. In this study, we examined the data of 271 non-demented memory clinic patients and found an additive association of $A \beta$ and WMH on medial temporal lobe atrophy. Regarding cognitive decline, we found that both pathologies were of influence, but when both present the effect were not stronger. These findings may have implications for the short-term prognosis of memory clinic patients.

To obtain a better understanding of the temporal pattern of pathologies involved in $\mathrm{AD}$ pathophysiology and how these might affect cognition, we examined how established and novel $\mathrm{AD}$ biomarkers are related to each other and to cognitive decline. In chapter 7 , we investigated in a combined dataset consisting of 907 cognitively normal individuals whether $A \beta$ and tau influenced cognitive test performance. We found that $A \beta$ negatively influenced performance on four neuropsychological tests, whereas we found no influence of tau on cognitive tests. Consequently, we created novel normative data for these four tests based on individuals without $A \beta$ pathology. For memory tests the novel norms yielded more sensitive in identifying individuals at risk of progression to dementia than traditional published norms. In the study described in chapter 8, we included 770 individuals across the clinical $A D$ spectrum and investigated the relationship between $A \beta$ status, $A P O E$ \& 4 carriership and cognition with novel cerebrospinal fluid markers of synaptic dysfunction (Neurogranin, Ng), axonal degeneration (Neurofilament-light, NFL) and astroglial activation (YKL-40). We showed that axonal degeneration, synaptic dysfunction and astroglial activation are involved in $\mathrm{AD}$ pathophysiology already in the preclinical stage and that all three markers are associated with cognitive decline. In chapter 9, we characterized individuals with MCI with AD-like neuronal injury but without evidence of amyloid pathology, who are often referred to as having Suspected Non-Alzheimer's Disease Pathophysiology (SNAP). We included 584 individuals with normal cognition or MCI from eight different cohorts in which we analyzed established and novel CSF biomarkers and performed cluster analyses on CSF markers to define SNAP subgroups. MCI-SNAP showed evidence of amyloid production as they had strongly increased levels 


\section{Addendum}

of non-aggregating forms of $A \beta$ isoforms in CSF, compared to the control group. Cluster analyses identified three SNAP subgroups that differed on the degree in neuronal activity. These findings suggest that MCI-SNAP is a heterogeneous condition that warrants further investigation.

Chapter 10, provides a general discussion of our findings, the implications for scientific research, prevention trials and clinical practice. 


\section{Nederlandse samenvatting}

Biomarkers hebben de kennis en het inzicht in de ziekte van Alzheimer (AD) vergroot. Desalniettemin begrijpen we nog niet alles rondom deze ziekte, met name omdat de tot nu toe uitgevoerde biomarker studies zich met name gericht op de klassieke pathologische kenmerken van $\mathrm{AD}$ : amyloid- $\beta(\mathrm{A} \beta)$ "plaques" en neurofibrillaire "tangles". In dit proefschrift hebben we onderzocht hoe vasculaire en neurodegeneratieve processen zich verhouden tot de traditionele AD biomarkers ( $\mathrm{A} \beta$ en tau) en cognitieve achteruitgang in de pre-dementie fases. Om dit op grote schaal te kunnen onderzoeken hebben we bestaande data hergebruikt en gecombineerd in het kader van het European Medical Information Framework for AD (EMIF-AD) project dat wordt beschreven in deel I. In deel II concentreerden we ons op de relatie tussen vasculaire risicofactoren, vasculaire pathologie en AD biomarkers. Deel III richt zich op traditionele en nieuwe AD biomarkers in het hersenvocht en hoe deze zich verhouden tot cognitieve achteruitgang.

Om het wetenschappelijk onderzoek rondom AD verder te helpen zijn krachtige en generaliseerbare statistische analyses nodig. Dergelijke analyses kunnen enkel uitgevoerd worden in grootschalige databases, welke gerealiseerd kunnen worden door het combineren van bestaande data. In hoofdstuk 2 beschreven we de EMIF-AD infrastructuur die als methodologische basis diende voor dit proefschrift. De EMIF-AD infrastructuur bevat een online catalogus met meta-data over cohorten, een dataplatform voor de opslag van geharmoniseerde subject-level data en de "AD-Switchbox" die gepoolde analyses op lokale databases mogelijk maakt. Hoofdstuk 3 richt zich op een grote studie binnen EMIFAD: "EMIF-AD Multimodal Biomarker Discovery" (EMIF-AD MBD) studie. In deze studie is data van 1221 oudere volwassenen van boven de 50 jaar, die oorspronkelijk onderdeel uitmaakten van 11 verschillende cohortstudies, samengevoegd. Klinische data is geharmoniseerd en MRI-scans, plasma-, DNA- en CSF-monsters zijn centraal geanalyseerd. De klinische data bevestigde de centrale rollen van $\mathrm{A} \beta$ en $A P O E \varepsilon 4$ in de pathogenese van AD. De geplande multimodale analyses uitgevoerd zullen nieuwe inzichten verstrekken over de onderliggende mechanismes van $\mathrm{AD}$.

Om de invloed van vasculaire aandoeningen op pre-dementie AD beter te begrijpen hebben we de relaties tussen vasculaire risicofactoren, vasculaire pathologie, $\mathrm{AD}$ biomarkers en cognitie onderzocht. In hoofdstuk 4 hebben we data van 14 cohortstudies gecombineerd wat resulteerde in een dataset met gegevens over 1394 personen met lichte cognitieve stoornissen (MCI). In deze studie toonden wij aan dat depressie, obesitas en hypercholesterolemie minder vaak voor kwamen bij personen met een hoog-risico-AD biomarker-profiel in vergelijking met personen met een laag-risico-AD biomarker-profiel. Geen van de tien onderzochte risicofactoren kwam vaker voor bij mensen met een hoog- 
risico-AD biomarker profiel. Verder vonden we dat alleen alcohol gebruik het risico op cognitieve achteruitgang vergrootte, onafhankelijk van AD biomarkers. Samen suggereren deze bevindingen dat vasculaire risicofactoren wellicht een beperkte invloed hebben in het MCI-stadium. Daarom hebben we vervolgens, zoals beschreven in hoofdstuk 5, een studie uitgevoerd naar de relatie tussen vasculaire risicofactoren, AD biomarkers en cognitie in cognitieve gezonde vrijwilligers. Voor deze studie hebben we data van 433 deelnemers van de longitudinale studies bij het Knight Alzheimer's Disease Research Center bij Washington University in St. Louis gebruikt. We toonden aan dat obesitas minder vaak voorkwam bij mensen met tau pathologie en dat cardiovasculaire aandoeningen vaker voorkwamen bij mensen met $A \beta$ en tau pathologie, in vergelijking met mensen met normale AD biomarkerwaarden. Verder vonden we dat hypertensie, gebrek aan obesitas en een hoge vasculaire risicoscore waren geassocieerd met een snellere toename van tau pathologie over de tijd, in het bijzonder bij mensen die al $\mathrm{A} \beta$ pathologie hadden. Gezien deze bevindingen zouden secundaire preventie strategieën voor $\mathrm{AD}$ zich kunnen richten op behandeling van deze factoren in de vroege fase van de ziekte. In hoofdstuk 6 hebben we ons gericht op de relatie tussen $A \beta$ en witte stof hyperintensiteiten (WMH), welke pathologische kenmerken van cerebrovasculaire aandoeningen zijn. In deze studie hebben we data van 271 geheugenpoli patiënten zonder dementie geanalyseerd, waaruit bleek dat zowel $\mathrm{A} \beta$ en WMH pathologie invloed hadden op atrofie van de mediale temporale hersenkwab en als ze beiden aanwezig waren dan werd de invloed versterkt. Wat betreft cognitieve achteruitgang vonden we dat zowel $\mathrm{A} \beta$ als WMH hierop een invloed hadden, maar ze versterkten elkaar niet. Deze bevindingen zouden implicaties kunnen hebben voor de korte termijn prognose van geheugenpoli patiënten.

Om het inzicht te vergroten wat betreft het temporele patroon van $\mathrm{AD}$ pathologieën en de mogelijke invloed op cognitie, hebben we onderzocht hoe traditionele en nieuwe AD biomarkers zich tot elkaar verhouden en tot cognitieve achteruitgang. In hoofdstuk 7 , hebben we in een gecombineerde dataset bestaande uit 907 cognitief gezonde ouderen, de invloed van $A \beta$ en tau op cognitieve prestaties onderzocht. We vonden dat $A \beta$ een negatieve invloed had op de prestaties bij vier neuropsychologische testen. Daarentegen vonden we geen invloed van tau op cognitie in deze vroege fase van de ziekte. Vervolgens hebben we onderzocht of normen voor cognitieve tests gebaseerd op mensen zonder $A \beta$ pathologie de vroege detectie van dementie zou kunnen verbeteren. Voor geheugentesten bleken de nieuwe normen gevoeliger in het identificeren van mensen met een risico op het ontwikkelen van dementie, in vergelijking met traditionele normen. In hoofdstuk 8 beschrijven we een studie waarbij data is gebruikt van 770 ouderen in verschillende cognitieve stadia van $\mathrm{AD}$ waarbij we de relatie tussen $\mathrm{A} \beta$ status, $A P O E \varepsilon 4$ dragerschap en cognitie met biomarkers in hersenvocht voor synaptische disfunctie (Neurogranin, Ng), axonale degeneratie (Neurofilament-light, NFL) en astrogliale activiteit (YKL-40) onderzochten. In deze studie toonden we aan dat de processen axonale degeneratie, synaptische 
dysfunctie en astrogliale activiteit al in de vroege, preklinische, fase van AD een rol spelen, alsmede dat alle drie deze processen een invloed hebben op cognitieve achteruitgang. In hoofdstuk 9 hebben we ons gericht op personen met MCI die AD gerelateerde neuronale schade hadden maar normale $\mathrm{A} \beta$ biomarkers hadden, een biomarker subtype dat kan worden aangeduid als 'Suspected Non-Alzheimer's Disease Pathophysiology' (SNAP). In deze studie hebben we 584 mensen met normale cognitie of MCI geïncludeerd van acht verschillende cohorten waarin we traditionele en nieuwe biomarkers in het hersenvocht hebben onderzocht en cluster analyses hebben uitgevoerd om SNAP-subtypes te definieren. We vonden dat MCI-SNAP zich kenmerkte door een sterk verhoogd niveau van niet-klonterende vormen van $A \beta$ isoformen in het hersenvocht, in vergelijking met de controlegroep. De cluster analyses identificeerden drie SNAP subgroepen die verschillenden in de mate van neuronale activiteit. Deze bevindingen laten zien dat MCI-SNAP een heterogene staat is die verder onderzoekt behoeft.

Hoofdstuk 10 biedt een algemene discussie van de resultaten van alle aparte hoofdstukken en de implicaties voor wetenschappelijk onderzoek, medicijnstudies en de klinische praktijk. 


\section{Knowledge valorization}

The knowledge resulting from the studies described in this thesis is not only of value to the scientific community but also has clinical and societal relevance. This paragraph addresses the importance of our studies and potential implementation opportunities in- and outside the academic setting.

\section{Relevance}

Dementia is a devastating disease which affects approximately 50 million people worldwide, a number that is expected to triple by 2050 due to the aging population. Alzheimer's disease (AD) is the most common form of dementia as it accounts for 60-80 percent of dementia cases. The disease places a heavy burden on patients, family and caregivers and due to the increasing prevalence, it has also become a major challenge for societies, economies and healthcare systems. In 2015, the global costs of dementia were estimated around 818 billion US dollars and this year, 2018, they are expected to cross the threshold of 1 trillion US dollars. Dementia is a problem that requires worldwide attention. This vision is supported by the Global Action Plan on the Public Health Response to Dementia of the World Health Organization (WHO) which stimulates governments to facilitate scientific research and optimize care for patients.

In current clinical practice, no disease modifying treatment for $\mathrm{AD}$ can be offered. Thus far, results from clinical trials examining potential drugs have been disappointing. A reason for this could be that the therapeutics are offered too late in the disease process when damage to the brain has become irreversible. Therefore, many studies now aim to identify individuals before they have $\mathrm{AD}$-type dementia in order to start interventions earlier. This thesis has also contributed to this effort as we created novel normative data based only on individuals without amyloid- $\beta(A \beta)$ which are more sensitive in identifying individuals at risk of developing dementia. On the other hand, the studies in this thesis underline the multifactorial nature of $\mathrm{AD}$ and thereby stress the importance of multifactorial secondary prevention. We showed that although vascular risk factors and vascular pathology do not have a direct effect on $A \beta$, they do have an effect on neurodegeneration and thereby possibly also on the rate of cognitive deterioration. In addition, our results suggest that processes like axonal degeneration, synaptic dysfunction and astroglial activation play a role already very early in $\mathrm{AD}$ and may have a profound effect on cognitive decline, making them potential targets for disease modifying treatments. Moreover, the studies in this thesis improve prognostic accuracy for current and future patients which can be extremely 
valuable for patients, caregivers and family members as well as for healthcare institutions and policymakers.

\section{Targeted audience}

The studies described in this thesis may be of relevance to a broad audience. First, our results are relevant for clinicians responsible for assessing and diagnosing individuals with cognitive problems. Our results may aid with the interpretation of biomarker and neuropsychological reports and improve prognostic accuracy. As clinicians could use our results to provide a more accurate prognosis for patients in various stages of the disease, our results are also, indirectly, relevant for patients and caregivers. Next, our results are also highly relevant for scientists as they increase understanding of $\mathrm{AD}$ pathophysiology and improve diagnostic and prognostic accuracy and provide a basis for future prevention studies. In addition, the data sharing infrastructure built as a methodological basis for the studies in this thesis is a powerful and valuable tool for future studies in the field of AD but also other disease areas. Fourth, our studies could be of relevance to pharmaceutical companies as they provide insight into mechanisms underlying AD which help to identify novel treatment targets, to select participants for trials and to identify outcome measures for clinical trials. Lastly, this thesis may also be of interest to policy makers as our studies underline the importance of research into $\mathrm{AD}$ as this increases the knowledge that is needed to find effective treatments for AD. For policy makers, it is also interesting to learn that a promising prevention strategy for $\mathrm{AD}$ may be to treat vascular factors which have shown to be associated with AD pathology.

\section{Products}

The infrastructure built as part of the European Medical Information Framework for AD (EMIF-AD) facilitates reuse and combining of existing data and is a product of the studies conducted in this thesis. The facilities created as part of EMIF-AD - the online catalogue, data platform and the $\mathrm{AD}$-Switchbox - are sustainable and can be utilized by researchers worldwide and could also be adapted to facilitate research in other disease areas. A special product resulting from EMIF-AD is the EMIF-AD Multimodal Biomarker Discovery (MBD) cohort. Because of its sample size and unique multimodal data collection, this cohort will be instrumental to study the early pathophysiology of AD in detail. Lastly, the normative data based on individuals without $A \beta$ will be valuable for clinical practice, research and for pharmaceutical companies as it increases the diagnostic accuracy of early AD. 


\section{Addendum}

\section{Innovation}

The studies described in this thesis were aimed to unravel several pathological mechanisms underlying $\mathrm{AD}$ beyond the involvement of the classic $\mathrm{AD}$ pathology in large sized studies. This provides a novel, multifactorial view of the disease and shows the involvement of different mechanisms along the course of the disease. Moreover, we studied new concepts such as atypical biomarker profiles (e.g. Suspected Non-Alzheimer's disease Pathophysiology, SNAP) and novel diagnostic and prognostic biomarkers. The methodological background of this thesis is unique as we used large datasets only by reusing and combining existing data. The data sharing infrastructure may set the standard for worldwide collaborations aimed to tackle major healthcare challenges such as finding effective treatment for $\mathrm{AD}$. In addition, we were among the first to study the influence of vascular risk factors on longitudinal cerebrospinal fluid (CSF) biomarkers, which provided support for possible causal relationships.

\section{Implementation}

The data sharing infrastructure developed as part of this thesis is already being used by a large number of researchers around the world and we have secured funding to sustain this infrastructure in the near future. Our results regarding vascular contributions to $\mathrm{AD}$ are now part of the scientific literature which can be used to shape prevention studies and trials for AD. In addition, in the Discussion chapter of this thesis we provided future directions regarding software that could aid to bridge the gap between research findings. The proposed software for clinicians which facilities storage and interpretation of elaborate patient profiles with respect to biomarkers, neuropsychological data and co-morbid disorders can be developed in a future scientific project. 


\section{Author affiliations}

\section{Pauline Aalten}

Department of Psychiatry and Neuropsychology, Maastricht University, School for Mental Health and Neuroscience, Alzheimer Center Limburg, Maastricht, the Netherlands

Daniel Alcolea

Hospital de la Santa Creu i Sant Pau, Barcelona, Spain

Myriam Alexander

PDB RWD (Real World Data) Team, Roche Products Limited, Welwyn Garden City, UK

Ulf Andreasson

Clinical Neurochemistry Lab, Institute of Neuroscience and Physiology, Sahlgrenska University Hospital, Mölndal, Sweden; Institute of Neuroscience and Physiology, Department of Psychiatry and Neurochemistry, University of Gothenburg, Mölndal, Sweden

Alison Baird

University of Oxford, Oxford, United Kingdom

Inês Baldeiras

Center for Neuroscience and Cell Biology, Faculty of Medicine, Department of Neurology, Centro Hospitalare Universitário de Coimbra, Coimbra, Portugal

Frederik Barkhof

Department of Radiology and Nuclear Medicine, VU University Medical Center, Amsterdam, The Netherlands; Institutes of Neurology and healthcare engineering, University College London, UK

Christine Bastin

GIGA-CRC in vivo Imaging, University of Liège, Liège, Belgium

Bart N. van Berckel

Department of Radiology and Nuclear Medicine, VU University Medical Center, Amsterdam, the Netherlands

Lars Betram

Lübeck Interdisciplinary Platform for Genome Analytics, University of Lübeck, Germany; School of Public Health, Imperial College London, UK; Department of Psychology, University of Oslo, Norway 


\section{Addendum}

Kaj Blennow

Clinical Neurochemistry Lab, Institute of Neuroscience and Physiology, Sahlgrenska University Hospital, Mölndal, Sweden; Institute of Neuroscience and Physiology, Department of Psychiatry and Neurochemistry, University of Gothenburg, Mölndal, Sweden

Olivier Blin

Mediterranean Institute of Cognitive Neuroscience, Aix Marseille University, Marseille, France

Régis Bordet

University of Lille, Inserm, CHU Lille, France

Anouk den Braber

Alzheimer Center and Department of Neurology, Neuroscience Campus Amsterdam, VU University Medical Center, Amsterdam, the Netherlands; Department of Biological Psychology, VU University Amsterdam, Amsterdam, the Netherlands

Christine Van Broekhoven

Neurodegenerative Brain Diseases group, VIB-Department of Molecular Genetics, Antwerp, Belgium; Institute Born-Bunge, University of Antwerp, Antwerp, Belgium

Mark. A. van Buchem

Department of Radiology, Leiden University Medical Center, Leiden, The Netherlands

Gaël Chételat

Inserm, Inserm UMR-S U1237, Université de Caen-Normandie, GIP Cyceron, Caen, France

Preciosa M. Coloma

Real World Data Science (RWD-S) Neuroscience and Established Products, F. HoffmannLa Roche Ltd. Pharmaceuticals Division, Basel, Switzerland

Solene Dauby

Department of Neurology and Memory Clinic, CHU Liège, Liège, Belgium

Mira Didic

AP-HM Hôpitaux de la Timone, Service de Neurologie et Neuropsychologie, Marseille, France; Aix-Marseille Université, INSERM, Institut de Neurosciences des Systèmes, Marseille, France

Valerija Dobricic

Lübeck Interdisciplinary Platform for Genome Analytics, University of Lübeck, Germany 
Richard Dobson

King's College London, London, United Kingdom; NIHR Biomedical Research Centre for Mental Health and Biomedical Research Unit for Dementia at South London and Maudsley NHS Foundation, London, United Kingdom; Farr Institute of Health Informatics Research, UCL Institute of Health Informatics, University College London, London, UK; NIHR University College London Hospitals Biomedical Research Centre

Alexander Drzezga

Department of Nuclear Medicine, University of Cologne, Cologne, Germany

Mirian Ecay-Torres

Center for Research and Advanced Therapies, CITA-Alzheimer Foundation, San Sebastian, Spain

Sebastiaan Engelborghs

Department of Neurology and Memory Clinic, Hospital Network Antwerp (ZNA) Middelheim and Hoge Beuken, Antwerp, Belgium, University of Antwerp, Antwerp, Belgium

Ainara Estanga

Center for Research and Advanced Therapies, CITA-Alzheimer Foundation, San Sebastian, Spain

Anne M. Fagan

Department of Neurology, Washington University School of Medicine, St. Louis, MO, USA; Knight Alzheimer's Disease Research Center, Washington University School of Medicine, St. Louis, MO, USA

Wiesje van der Flier Alzheimer Center, VU University Medical Center, Amsterdam, the Netherlands

Yvonne Freund-Levi

Department of Neurobiology, Caring Sciences and Society (NVS), Division of Clinical Geriatrics, Karolinska Institutet, and Department of Geriatric Medicine, Karolinska University Hospital Huddinge, Stockholm, Sweden; Department of Psychiatry Norrtälje Hospital Tiohundra, Norrtälje, Sweden

Giovanni B. Frisoni

Geneva Neuroscience Center, University Hospital and University of Geneva, Geneva, Switzerland; IRCCS San Giovanni di Dio Fatebenefratelli, Brescia, Italy

Lutz Froelich

Department of Geriatric Psychiatry, Zentralinstitut für Seelische Gesundheit, University of Heidelberg, Mannheim, Germany 


\section{Addendum}

Silvy Gabel

Laboratory for Cognitive Neurology, Department of Neurosciences, KU Leuven, Belgium; Alzheimer Research Centre, KU Leuven, Belgium

Samantha Galluzzi

IRCCS San Giovanni di Dio Fatebenefratelli, Brescia, Italy

Mark Forrest Gordon

Boehringer Ingelheim Pharmaceuticals, Inc, Ridgefield, CT, USA

Elizabeth Grant

Knight Alzheimer's Disease Research Center, Washington University School of Medicine, St. Louis, MO, USA; Division of Biostatistics, Washington University School of Medicine, St. Louis, MO, USA

Harald Hampel

Sorbonne Universités, Université Pierre et Marie Curie, Paris 06, AXA Research Fund \& UPMC Chair, Institut de la Mémoire et de la Maladie d'Alzheimer (IM2A) \& Institut du Cerveau et de la Moelle épinière (ICM), Département de Neurologie, Hôpital de la PitiéSalpétrière, Paris, France

Jason Hassenstab

Department of Neurology, Washington University School of Medicine, St. Louis, MO, USA; Knight Alzheimer's Disease Research Center, Washington University School of Medicine, St. Louis, MO, USA

Sanna-Kaisa Herukka

Institute of Clinical Medicine, Neurology, University of Eastern Finland and Neurocenter, Neurology, Kuopio University Hospital, Kuopio, Finland

Nigel Hughes

Janssen Research and Development, Beerse, Belgium

Heidi I.L. Jacobs

Department of Psychiatry and Neuropsychology, Maastricht University, School for Mental Health and Neuroscience, Alzheimer Center Limburg, Maastricht, the Netherlands

Willemijn J. Jansen

Department of Psychiatry and Neuropsychology, Maastricht University, School for Mental Health and Neuroscience, Alzheimer Center Limburg, Maastricht, the Netherlands

Peter Johannsen

Danish Dementia Research Centre, Rigshospitalet, Copenhagen University Hospital, Copenhagen, Denmark 
Mara ten Kate

Alzheimer Center and Department of Neurology, VU University Medical Center, Amsterdam, the Netherlands

Petronella Kettunen

University of Gothenburg, Sahlgrenska Academy, Institute of Neuroscience and Physiology, Section for Psychiatry and Neurochemistry, Gothenburg, Sweden; University of Oxford, Nuffield Department of Clinical Neurosciences, Oxford, United Kingdom

Johannes Kornhuber

Department of Psychiatry and Psychotherapy, Friedrich-Alexander University of ErlangenNürnberg, Erlangen, Germany

Cristina Legido-Quigley

Steno Diabetes Center, Copenhagen, Denmark; King's College London, London, United Kingdom

Alberto Lleó

Hospital de la Santa Creu i Sant Pau, Barcelona, Spain

Simon Lovestone

University of Oxford, Oxford, United Kingdom

Wolfgang Maier

Department of Psychiatry and Psychotherapy, University of Bonn, German Center for Neurodegenerative Diseases (DZNE), Bonn, Germany

Pablo Martinez-Lage

Center for Research and Advanced Therapies, CITA-Alzheimer Foundation, San Sebastian, Spain

Alexandre de Mendonça

Institute of Molecular Medicine and Faculty of Medicine, University of Lisbon, Portugal

José Luis Molinuevo

Alzheimer's disease \& other cognitive disorders unit, Hopsital Clínic-IDIBAPS, Barcelona, Spain; Barcelona Beta Brain Research Center, Unversitat Pompeu Fabra, Barcelona, Spain

Silvia Morbelli

Nuclear Medicine, Department of Health Science (DISSAL), University of Genoa IRCCS AOU San Martino-IST, Genoa, Italy 


\section{Addendum}

John C. Morris

Department of Neurology, Washington University School of Medicine, St. Louis, MO, USA;

Knight Alzheimer's Disease Research Center, Washington University School of Medicine,

St. Louis, MO, USA

Ellis Niemantsverdriet

Reference Center for Biological Markers of Dementia (BIODEM), University of Antwerp, Antwerp, Belgium

Flavio Nobili

Clinical Neurology, Department of Neurosciences (DINOGMI), University of Genoa and IRCCS AOU San Martino-IST, Genoa, Italy

Arto Nordlund

Department of Psychiatry and Neurochemistry, Institute of Neuroscience and Physiology,

Sahlgrenska Academy, University of Gothenburg, Gothenburg, Sweden

Gerald P. Novak

Janssen Pharmaceutical Research and Development, Titusville, NJ, USA

Marcel Olde Rikkert

Radboudumc Alzheimer Centre, Department of Geriatric Medicine, Radboud University Medical Center, Nijmegen, The Netherlands.

Ania Oleksik

Department of Gerontology and Geriatrics, Leiden University Medical Center, Leiden, The Netherlands

José Luis Oliveira

Institute of Electronics and Informatics Engineering, University of Aveiro, Portugal

Oliver Peters

Department of Psychiatry and Psychotherapy, Charité Berlin, Berlin, Germany

Gwendoline Peyratout

Department of Psychiatry, University Hospital of Lausanne, Lausanne, Switzerland

Julius Popp

Geriatric Psychiatry, Department of Mental Health and Psychiatry, Geneva University Hospitals, Switzerland; Department of Psychiatry, University Hospital of Lausanne, Lausanne, Switzerland

Inez Ramakers

Department of Psychiatry and Neuropsychology, Maastricht University, School for Mental Health and Neuroscience, Alzheimer Center Limburg, Maastricht, the Netherlands 
Lorena Rami

Alzheimer's disease \& other cognitive disorders unit, Hopsital Clínic-IDIBAPS, Barcelona, Spain

Jill Richardson

Neurosciences Therapeutic Area, GlaxoSmithKline R\&D, Stevenage, UK

Ellen Elisa De Roeck

Reference Center for Biological Markers of Dementia (BIODEM), University of Antwerp, Antwerp, Belgium; Department of Clinical and Lifespan Psychology, Vrije Universiteit Brussel, Brussels, Belgium

Eckhart Rüther

Department of Psychiatry and Psychotherapy, University of Göttingen, Göttingen, Germany

Isabel Sala

Department of Neurology, Hospital de la Santa Creu i Sant Pau, Barcelona, Spain

Eric Salmon

Department of Neurology and Memory Clinic, CHU Liège, Liège, Belgium; GIGA-CRC in vivo Imaging, University of Liège, Liège, Belgium

Isabel Santana

Center for Neuroscience and Cell Biology, Faculty of Medicine, Department of Neurology, Centro Hospitalar e Universitário de Coimbra, Coimbra, Portugal

Vincente de la Sayette

Inserm, Inserm U1077, Université de Caen Normandie, Ecole Pratique des Hautes Etudes, Caen, France; CHU de Caen, Service de Neurologie, Caen, France

Philip Scheltens

Alzheimer Center and Department of Neurology, VU University Medical Center, Amsterdam, the Netherlands

Suzanne Schindler

Department of Neurology, Washington University School of Medicine, St. Louis, MO, USA; Knight Alzheimer's Disease Research Center, Washington University School of Medicine, St. Louis, MO, USA

Janneke Schoots - van der Ploeg

The Hyve, Utrecht, the Netherlands

Dina Silva

Institute of Molecular Medicine and Faculty of Medicine, University of Lisbon, Portugal 


\section{Addendum}

Kristel Sleegers

Neurodegenerative Brain Diseases group, VIB-Department of Molecular Genetics, Antwerp, Belgium; Institute Born-Bunge, University of Antwerp, Antwerp, Belgium

Hilkka Soininen

Institute of Clinical Medicine, Neurology, University of Eastern Finland and Neurocenter, Neurology, Kuopio University Hospital, Kuopio, Finland

Michel Van Speybroeck

Janssen Research and Development, Beerse, Belgium

Johannes Streffer

Early Clinial Neurology, UCB Biopharma SPRL, Belgium; Reference Center for Biological Markers of Dementia (BIODEM), Institute Born-Bunge, University of Antwerp, Antwerp, Belgium

Mikel Tainta

Center for Research and Advanced Therapies, CITA-Alzheimer Foundation, San Sebastian, Spain

Charlotte Teunissen

Alzheimer Center, VU University Medical Center, Amsterdam, the Netherlands

Jori Tomassen

Alzheimer Center, VU University Medical Center, Amsterdam, the Netherlands

Magda Tsolaki

$1^{\text {st }}$ Department of Neurology, AHEPA University Hospital, Makedonia, Thessaloniki, Greece

Rik Vandenberghe

University Hospital Leuven, Leuven, Belgium; Laboratory for Cognitive Neurology, Department of Neurosciences, KU Leuven, Belgium

Bart Vannieuwenhuysen

Janssen Research and Development, Beerse, Belgium

Rudi Verbeeck

Janssen Research and Development, Beerse, Belgium

Marcel M. Verbeek

Departments of Neurology and Laboratory Medicine, Donders Institute for Brain, Cognition and Behaviour, Radboud Alzheimer Center, Radboud University Medical Center, Nijmegen, The Netherlands 
Frans R.J. Verhey

Department of Psychiatry and Neuropsychology, Maastricht University, School for Mental Health and Neuroscience, Alzheimer Center Limburg, Maastricht, the Netherlands

Pieter Jelle Visser

Department of Psychiatry and Neuropsychology, Maastricht University, School for Mental Health and Neuroscience, Alzheimer Center Limburg, Maastricht, the Netherlands

Stephanie Vos

Department of Psychiatry and Neuropsychology, Maastricht University, School for Mental Health and Neuroscience, Alzheimer Center Limburg, Maastricht, the Netherlands

Anders Wallin

University of Gothenburg, Sahlgrenska Academy, Institute of Neuroscience and Physiology, Section for Psychiatry and Neurochemistry, Gothenburg, Sweden

Åsa K. Wallin

Department of Clinical Sciences Malmö, Lund University, Clinical Memory Research Unit, Lund, Sweden

Sarah Westwood

University of Oxford, Oxford, United Kingdom

Angelika Wientzek

PDB RWD (Real World Data) Team, Roche Products Limited, Welwyn Garden City, UK; Epidemiologische Beratung und Literatur-Recherche "conepi”, Herrsching, Germany Jens Wiltfang

Department of Psychiatry and Psychotherapy, University Medical Center (UMC), GeorgAugust-University, Göttingen, Germany

Chengjie Xiong

Knight Alzheimer's Disease Research Center, Washington University School of Medicine, St. Louis, MO, USA; Division of Biostatistics, Washington University School of Medicine, St. Louis, MO, USA

Henrik Zetterberg

Clinical Neurochemistry Lab, Institute of Neuroscience and Physiology, Sahlgrenska University Hospital, Mölndal, Sweden; Institute of Neuroscience and Physiology, Department of Psychiatry and Neurochemistry, University of Gothenburg, Mölndal, Sweden; Department of Molecular Neuroscience, UCL Institute of Neurology, London, UK; UK Dementia Research Institute, London, UK. 


\section{List of publications}

\section{Thesis}

Bos, I., Vos, S.J., Fröhlich, L., Kornhuber, J., Wiltfang, J., Maier, W., Peters, O., Rüther, E., Engelborghs, S., Niemantsverdriet, E., De Roeck, E.E., Tsolaki, M., Freund-Levi, Y., Johannsen, P., Vandenberghe, R., Lleó, A., Alcolea, D., Frisoni, G.B., Galluzzi, S., Nobili, F., Morbelli, S., Drzezga, A., Didic, M., Berckel van, B., Salmon, E., Bastin, C., Dauby, S., Santana, I., Balderias, I., Mendonça, A., Silva, D., Wallin, A., Nordlund, A., Coloma, P.M., Wientzek, A., Alexander, M., Novak, G.P., Gordon, M.F., the Alzheimer's Disease Neuroimaging Initiative, Wallin, A.K., Hampel, H., Soininen H., Herukka, S., Scheltens, P., Verhey, F., Visser, P.J. The frequency and influence of dementia risk factors in prodromal Alzheimer's disease. Neurobiology of Aging. 2017; 56, 33-40

Bos, I., Verhey, F., Ramakers, I., Jacobs, H.I.L., Soininen, H., Freund-Levi, Y., Hampel, H., Tsolaki, M., Wallin, A. K., van Buchem, M. A., Oleksik, A., Verbeek, M. M., Olde Rikkert, M., van der Flier, W. M., Scheltens, P., Aalten, P., Visser, P. J., Vos, S. J. B. Cerebrovascular and amyloid pathology in pre-dementia stages: the relationship with neurodegeneration and cognitive decline. Alzheimer Research \& Therapy. 2017; 9(1), 101

Bos, I., Vos, S.J., Vandenberghe, R., Scheltens, P, Engelborghs, S., Frisoni, G., Molinuevo, J., Wallin, A., Lléo, A., Popp, J., Martinez-Lage, P., Baird, A., Dobson, R., Legido-Quigley, C., Bertram, L., Sleegers, K., Kate ten, M., Barkhof, F., Zetterberg, H., Lovestone, S., Streffer, J., Visser, P. The EMIF-AD Multimodal Biomarker Discovery Study: Design, methods and cohort characteristics. Alzheimer Research \& Therapy. 2018

Bos, I., Vos, S.J., Jansen, W.J., Vandenberghe, R., Estanga, A., Ecay-Torres, M., Tomassen, J., Braber den A., Lleó, A., Sala., I., Wallin., A., Kettunen, P., Molinuevo, J.L., Rami, L., Chetelat, G., Sayette de la., V., Tsolaki, M., Freund-Levi, Y., Johannsen, P., the Alzheimer's Disease Neuroimaging Initiative, Novak, G., Ramakers, I., Verhey, F., Visser, P.J. A $\beta$, tau and cognition in cognitively normal individuals: Examining the necessity to adjust for biomarker status in normative data. Frontiers in Aging Neuroscience. 2018; 10:193

Bos, I., Vos, S.J., Schindler, S., Hassenstab, J., Xiong, C., Grant, E., Verhey, F., Morris, J.C., Visser, P.J., Fagan, A.M. Vascular risk factors are associated with cerebrospinal fluid biomarker changes and cognition in preclinical Alzheimer's disease. Submitted. 
Bos, I., Vos, S.J., Verhey, F., Scheltens, P., Teunissen, C., Engelborghs, S., Sleegers, K., Frisoni, G., Blin, O., Richardson, J., Bordet, R., Tsolaki, M., Popp, J., Peyratout, P., Martinez-Lage, P., Tainta, M., Lleó, A., Johannsen, P., Freund-Levi, Y., Frölich, L., Vandenberghe, R., Westwood, S., Dobricic, V., Barkhof, F., Legido-Quigley, C., Bertram, L., Lovestone, S., Streffer, J., Andreasson, U., Blennow, K., Zetterberg, H., Visser, P.J. Cerebrospinal fluid biomarkers of neurodegeneration, synaptic dysfunction and astroglial activation in relation to amyloid- $\beta$, APOE genotype and cognition across the Alzheimer's disease spectrum. Submitted.

Vos, S.J., Bos, I., Verhey, F., Engelborghs, S., Sleegers, K., Lleó A., Johannsen, P., FreundLevi, Y., Frölich, L., Vandenberge, R., Frisoni, G., Blin, O., Richardson, J., Bordet, R., Scheltens, P., Ramakers, I., Popp, J., Tsolaki, M., Martinez-Lage, P., Bjerke, M., Teunissen, C., Peyratout, G., Tainta, M., Dobricic, V., Bertram, L., Lovestone, S., Streffer, J., Blennow, K., Andreasson, U., Zetterberg, H., Visser, P.J. Increased cerebrospinal fluid amyloid- $\beta 40$ and 38 concentrations in individuals with mild cognitive impairment and Suspected NonAlzheimer's Disease Pathophysiology. Submitted.

\section{Other}

Kate ten, M., Redolfi, A., Bos, I., Vos, S.J.B., Vandenberghe, R., Gabel, S., Schaeverbeke, J., Scheltens, P., Blin, O., Richardson, J., Bordet, R., Wallin, A., Eckerstrom, C., Molineuvo, J.L., Engelborghs, S., Broeckhoven van, C., Martinez-Lage, P., Popp, J., Tsolaki, M., Verhey, F., Baird, A.L., Legido-Quigley, C., Bertram, L., Dobricic, V., Zetterberg, H., Lovestone, S., Streffer, J., Bianchetti, S., Novak, G., Revillard, J., Gordon, M.F., Xie, Z., Wottschel, V., Frisoni, G., Visser, P.J., Barkhof, F. MRI predictors of amyloid pathology: results from the EMIF-AD Multimodal Biomarker Discovery study. Submitted. 


\section{Author information}

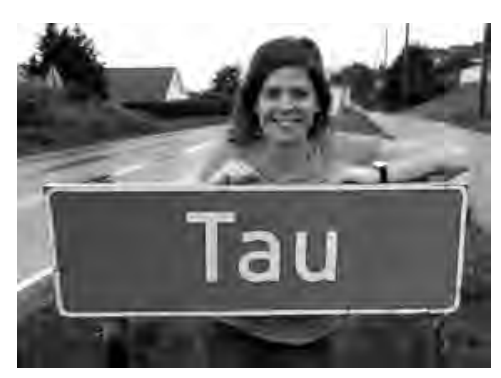

Isabelle Bos was born on April 26 1990 in Groningen, the Netherlands. After graduating from high school (Rölingcollege, Groningen) in 2007, she obtained her BSc degree in Psychology at the Rijksuniversiteit Groningen in 2012. Thereafter she obtained her MSc degree in Healthcare Psychology with a specialization in Clinical Neuropsychology at the University of Amsterdam in 2013. During her master, Isabelle did a clinical internship at the memory clinic of the hospital Ziekenhuis Gelderse Vallei in Ede and a research internship at the Max Planck Institute for Psycholinguistics in Nijmegen. In May 2014 Isabelle started working as a PhD candidate at the department of Psychiatry \& Neuropsychology at Maastricht University under supervision of Dr. Pieter Jelle Visser, Prof. dr. Frans Verhey, and Dr. Stephanie Vos. During her PhD, Isabelle visited the department of Neurology at Washington University School of Medicine in St. Louis, USA, for several months for a collaboration with Dr. Anne Fagan and colleagues. Currently, Isabelle is working as a postdoctoral researcher at the department of Psychiatry \& Neuropsychology of Maastricht University.

Isabelle Bos werd geboren op 26 april 1990 in Groningen, Nederland. In 2007 behaalde zij haar vwo-diploma (Rölingcollege, Groningen) en vervolgens in 2012 haar bachelor in de psychologie aan de Rijksuniversiteit Groningen. Daarna heeft zij in 2013 haar masterstudie in Gezondheidszorgpsychologie met als specialisatie Klinische Neuropsychologie aan de Universiteit van Amsterdam met succes voltooid. Tijdens haar master heeft Isabelle een klinische stage afgerond op de geheugenpoli van het Ziekenhuis Gelderse Vallei te Ede en een wetenschappelijke stage bij het Max Planck Insitute of Psycholinguistics in Nijmegen. In mei 2014 begon Isabelle met werken als promovendus op de afdeling Psychiatrie \& Neuropsychologie van Maastricht University onder supervisie van Dr. Pieter Jelle Visser, Prof. dr. Frans Verhey en Dr. Stephanie Vos. Tijdens haar promotietraject werkte zij enkele maanden op de afdeling Neurologie van Washington University, School of Medicine in St. Louis, V.S., in het kader van een samenwerking met Dr. Anne Fagan en collega's. Momenteel werkt Isabelle als postdoc onderzoeker op de afdeling Psychiatrie \& Neuropsychologie van Maastricht University. 


\section{Dankwoord}

En dan ineens is het zover: mijn proefschrift is echt af! De afgelopen vier jaar ben ik elke dag (nou ja, bijna elke dag dan) met veel plezier aan het werk gegaan en dat is mede dankzij de fijne mensen die om mij heen staan. Daarom wil ik nu een momentje nemen om deze mensen te bedanken.

Allereerst wil ik mijn promotieteam bedanken bestaande uit: dr. Pieter Jelle Visser, prof. dr. Frans Verhey en dr. Stephanie Vos.

Pieter Jelle, op het allerlaatste moment ben jij toch nog mijn eerste promotor geworden! Bedankt voor je enthousiasme en kennis over wetenschappelijk onderzoek en voor jouw kritische en ongezouten mening over van alles en nog wat. Ik waardeer je oprechtheid en ondanks dat ik moest wennen aan je cryptische mails en aanwijzingen, heb ik veel plezier beleefd aan onze samenwerking. Natuurlijk ook bedankt voor de pindakaasvrije dinsdagen! Frans, bedankt voor je leidinggevende rol in mijn promotieteam. Jij wist altijd op een soepele manier onze vaak ongestructureerde overleggen zo te sturen dat de belangrijkste conclusies op tafel kwamen te liggen. Bedankt voor het vertrouwen en de ruimte die je me gaf om mezelf te ontwikkelingen tot een volwaardig onderzoeker.

Stephanie, bedankt dat jij er altijd voor me was als ik het nodig had en de nauwkeurigheid inbracht die ik soms miste. Of het nou via de mail (meer dan 2700 mails in 4 jaar), skype, of in het echt was, we zijn een goed team! Dankzij jou ga ik de Vlaamse woorden 'ingeduffeld' en 'klommellen' met veel plezier gebruiken.

Mijn hele promotieteam wil ik hartelijk bedanken dat jullie me mee namen naar veel verschillende congressen, symposia en meetings waardoor jullie mij toelieten in jullie brede wetenschappelijke netwerken.

Mijn dank gaat ook uit naar alle patiënten en mantelzorgers voor hun deelname aan onze studies. Zonder jullie bijdrage was veel van het uitgevoerde onderzoek niet mogelijk geweest en daarbij zijn jullie een inspiratiebron voor vele onderzoekers.

I would also like to thank the members of the reading committee for reading and approving this thesis.

EMIF-AD colleagues, thank you for all the calls, meetings and conferences and for such an exciting and productive collaboration. I think we have done a great job during this project and it felt awesome to be working alongside all of you as a junior researcher! Nog een speciaal bedankje voor de Amsterdamse EMIFers voor de gezelligheid op congressen en de fijne samenwerking. 


\section{Addendum}

Co-authors \& data-providers, thank you for contributing data, knowledge and feedback. Your positive and encouraging responses from all over the world make data sharing and doing research much more fun!

Prof. dr. Fagan, dear Anne, thank you for welcoming me with open arms into your research group for several months and for such a great collaboration. I still don't understand how you are able to work on so many projects and still manage to reply to all my emails within 5 minutes! Julia, Suzanne, Courtney, Matt and Jason, thank you for showing me around WashU and St. Louis!

Alieske en Whitney, mijn lieve paranimfen, zonder jullie was het hele promotieavontuur een stuk minder leuk geweest! Al 3.5 jaar zijn we kamergenootjes en hebben we lief en leed met elkaar gedeeld. Lieve Alieske, je bent een bijzonder persoon en je hebt vaak zelf niet door hoe grappig je bent. Ik ben blij dat ik altijd bij jou terecht kan, of het nou is om een nachtje te blijven slapen, een kroket te eten, een nieuw spreekwoord te leren of gezellig te kletsen: je bent er altijd. Lieve Whitney, jij bent een van de weinige mensen die altijd om mijn flauwe grapjes lacht, maar ondertussen ook heel kritisch en serieus advies kan geven over een statistische analyse. Ik ben blij en trots dat jullie achter mij staan, nu (letterlijk) en in de toekomst. Hopelijk mogen er nog vele barbecues, vakanties en hijgende hert bezoekjes volgen!

Natuurlijk wil ik alle (oud)-collega's van de afdeling Psychiatrie en Neuropsychologie bedanken voor de gezelligheid en collegialiteit, mede dankzij jullie ga ik nog altijd met veel plezier naar mijn werk. Er zijn echter een paar (oud)-collega's die ik extra in het zonnetje wil zetten:

Willemijn, misschien komt het wel doordat mensen ons altijd door elkaar halen of omdat we gewoon goed op één lijn liggen, maar ik heb bij jou het "grote-zus-gevoel". Vanaf het eerste moment stond jij voor mij klaar (op mijn 2e werkdag wachtte jij me op bij het station!) met advies en tips, maar bovenal met een goeie dosis Brabantse humor. Lotte, in jou heb ik mijn mede-activist gevonden. Wij versterken elkaar in het voor jezelf opkomen en het schoppen tegen het systeem als iets, volgens ons, oneerlijk verloopt. Ik waardeer je enthousiasme, maar ook je Twentse nuchterheid. Zoals we al vaak hebben gezegd het afgelopen jaar: ik ben heel benieuwd waar we allemaal terecht gaan komen. Syenna, er is niemand zoals jij! Onze zwemsessies waren vaak veel te kort aangezien daar alles besproken moest worden: van allerhande recepten, ik-vertrek afleveringen tot sollicitatiegesprekken. Ik hoop dat we elkaar nog vaak blijven zien! Babette, heel erg fijn dat wij altijd bij elkaar naar binnen konden lopen met vragen over de meest uiteenlopende onderwerpen. Ik ben benieuwd wat de toekomst je gaat brengen en ik ben natuurlijk stiekem heel trots dat je je dochter Isabelle hebt genoemd. Elles, ik denk dat jij de meest efficiënte onderzoeker (en persoon?) bent die ik ken! 
Bedankt dat ik af en toe mocht meegenieten van je efficiëntie en voor de gezelligheid op werk of congressen. Mede treinreizigers (Whitney, Willemijn, Elles en Angelique), de tripjes naar huis zijn altijd gezelliger met jullie erbij! Joany, mijn mede aio-vertegenwoordiger en ex-buurvrouw, bedankt voor de gezelligheid en heel veel succes in Rotjeknor. Nico en Ron, de redders in nood! Heel erg bedankt dat jullie meerdere malen mijn computer hebben gereanimeerd en voor alle technische support én de laatste nieuwtjes. Astrid, Danielle en Els bedankt voor de administratieve ondersteuning, maar ook voor de fijne praatjes in de wandelgangen.

Andrea, Anne, Caithlin, Mathyza, Ninke, Renée, Renske, Roos en Tim, oftwel de kippers: bedankt voor de nodige afleiding en voor jullie onvoorwaardelijke vriendschap! Anniek, mijn festivalmaatje, ook al zien we elkaar niet elke dag als ik jou weer zie voelt het alsof er geen tijd tussen heeft gezeten. Ook al mijn andere lieve vrienden waarvan ik de namen niet durf op te schrijven omdat ik bang ben dat ik dan iemand vergeet: bedankt voor de leuke weekendjes, bejaardenuitjes, hulp bij verhuizingen (binnenkort weer? (-)), bezoekjes, bruiloften en etentjes!

Lieve oma's, wat ben ik blij dat jullie mijn gehele promotietraject hebben kunnen meemaken. Vaak heb ik vol trots op de afdeling verteld dat ik twee oma's van boven de 80 heb die heel goed overweg kunnen met computers en WhatsApp! Ook de rest van mijn familie natuurlijk heel erg bedankt voor de interesse in mijn onderzoek en de enthousiaste reacties bij iedere bereikte mijlpaal.

Lieve schoonfamilie, de afgelopen tien jaar zijn jullie onmisbaar geworden voor mij. Ondanks dat we zullen moeten afwachten wat de toekomst gaat brengen, hoop ik nog lang te kunnen genieten van al jullie liefde, steun en gezelligheid. Christian, succes met het afronden van jouw proefschrift. Ik weet zeker dat het gaat lukken en zoals je zelf ooit zei: als je er fulltime aan had kunnen werken was het allang af geweest!

Lieve Colette, Co, Kaas, stiekem zit jij altijd ergens in mijn hoofd. Een stemmetje dat mij motiveert of kritisch advies geeft. Je bent een geweldige grote zus en jouw gedrevenheid, passie en geloof in alles, maar zeker ook in mij, geven me de energie en het vertrouwen dat ik soms nodig heb. Iedereen heeft een zus nodig zoals jij!

Papa, met jou als voorbeeld heb ik geleerd dat hoogleraren ook maar gewoon mensen zijn en dat je een uitstekende onderzoeker kunt zijn en toch hard kan lachen om Scary Movie 3. Soms schoot je even in de rol van extra promotor door iets te vaak te vragen hoe het ervoor stond met mijn artikelen, maar gelukkig gaf jij me de ruimte en het vertrouwen om het zelf te doen. Mama, er is niks dat ik niet met jou kan bespreken! Ook al is het de afgelopen jaren meestal door de telefoon (vooral het becommentariëren van onze favoriete tv-programma's!) aangezien de afstand Roermond/Den Bosch - Woltersum niet zomaar te overbruggen is. Bedankt dat jullie er altijd voor me zijn! 


\section{Addendum}

Als allerlaatste degene die ik het meest wil bedanken van allemaal! Lieve Ardi, er is zoveel waar ik jou voor kan bedanken, dat het moeilijk is om op te schrijven. Met je nuchtere en realistische (of pessimistische?) kijk op het leven, breng je mij rust als ik het nodig heb, luister je altijd naar al mijn verhalen en weet je wat ik moet doen als ik het zelf even niet meer weet. Bedankt dat je met mij en onze boze konijn naar het verre zuiden wou verhuizen en voor de lol die we altijd samen hebben. Na 10 jaar weet ik zeker dat wij samen écht oud gaan worden, ondanks dat we nu al bejaard zijn vanbinnen. 


\section{Thesis defenses from MHeNs - School for Mental Health and Neuroscience}

\section{3}

Rob Havermans: Bipolar disorder in daily life; Mood and cortisol responses to naturally occurring events. Supervisor: Prof.dr. M. de Vries; CoSupervisor: Dr. N. Nicolson.

Véronique Moers-Hornikx: Deep brain stimulation and the cerebellum. Supervisors: Prof.dr. J. Vles / Prof.dr. Y. Temel; Co-Supervisor: Dr. G. Hoogland.

Nicole Veldhorst-Janssen: Intranasal delivery of rapid acting drugs. Supervisors: Prof.dr. M. Marcus / Prof.dr. C. Neef; Co-Supervisor: Dr. P.H. van der Kuy.

Stéphanie Knippenberg: Vitamin D and Multiple Sclerosis: immunological and clinical outcome. Supervisor: Prof.dr. J. Cohen-Tervaert; Co-Supervisors: Dr. J. Damoiseaux / Dr. Y. Bols.

Erik D. Gommer: Dynamic Cerebral Autoregulation: from methodology towards clinical application. Supervisors: Prof.dr. W.H. Mess / Prof.dr. R.B. Panerai, UK; Co-Supervisor: Dr.ir. J.P.H. Reulen.

Olga A.H. Reneerkens: Can PDE inhibition improve cognition? Translationalinsights. Supervisor: Prof.dr. H.W.M. Steinbusch; Co-Supervisor: Dr. J. Prickaerts.

Lyzel S. Elias-Sonnenschein: Clinical and biomarker correlates of genetic risk factors for Alzheimer's disease. Supervisor: Prof.dr. F.R.J. Verhey; Co-Supervisor: Dr. P.J. Visser.

Diego F. Mastroeni: Epigenetic Dysregulation and the Pathophysiology of Alzheimer's Disease. Supervisors: Prof.dr.H.W.M.Steinbusch/Prof.dr.P.D.Coleman, SunCity, Arizona; Co-Supervisors: Dr. B.P.F. Rutten / Dr. D.L.A. van den Hove.

Leonidas Chouliaras: Epigenetic Regulation in Aging and Alzheimer's disease: A translational perspective. Supervisor: Prof.dr. H.W.M. Steinbusch; Co-Supervisors: Dr. B.P.F. Rutten / Dr. D.L.A. van den Hove.
Liesbeth Knaepen: Perinatal events and altered pain sensitivity in later life. Supervisors: Prof.dr. E.A.J. Joosten / Prof.dr. D. Tibboel, EUR; Co-Supervisor: Dr. J. Patijn.

Marisela Martinez-Claros: Hippocampal plasticity and corticosterone: From dendrites to behaviour. Supervisor: Prof.dr. H.W.M. Steinbusch; CoSupervisors: Dr. J.L. Pawluski / Dr. J. Prickaerts.

Marcus D. Lancé: A circle of improvement in bleeding management: from laboratory to clinic and back. Supervisors: Prof.dr. M.A.E. Marcu / Prof.dr. J.W.M. Heemskerk; Co-Supervisor: Dr. Y.M.C. Henskens.

Hilde Braakman: Imaging the brain; neuronal correlates of cognitive impairment in children with frontal lobe epilepsy. Supervisors: Prof.dr.A.P. Aldenkamp /Prof.dr. J.S.H. Vles; Co-Supervisors: Dr.ir. W.H. Backes / Dr. P.A.M. Hofman.

Willem H. van Zwam: Aneurysmal subarachnoid hemorrhage: imaging strategies and cost-effectiveness aspects in diagnostic work-up and posttherapeutic follow-up. Supervisors: Prof.dr.J.T.Wilmink/Prof.dr.J.E.Wildberger; CoSupervisor: Dr. P.A.M. Hofman.

Klara De Cort: The Pathogenesis of Panic Disorder. Supervisors: Prof.dr. I. Myin-Germeys / Prof.dr. E.J.L. Griez; Co-Supervisors: Dr. K.R.J. Schruers / Dr. I. Van Diest, Leuven.

Kim van Wijck: Mind the Gap; experimental studies on splanchnic hyperfusion and gastrointestinal integrity loss in man. Supervisors: Prof.dr. W.A. Buurman /

Prof.dr. C.H.C. Dejong; Co-Supervisor: Dr. K. Lenaerts.

Yvette Roke: Antipsychotic-induced hyperprolactinemia in children and adolescents with mainly autism spectrum disorders. Prevalence, symptoms, clinical consequences and genetic risk factors. Supervisors: Prof.dr. P.N. van Harten / Prof.dr. J.K. Buitelaar (RUN); Co-Supervisor: Dr. A. Boot (UMCG). 
Fleur Goezinne: Retinal detachment surgery: preand postoperative prognostic factors. Supervisors: Prof.dr. F. Hendrikse / Prof.dr. C.A.B. Webers; Co-Supervisor:

Dr. E.C. La Heij (Amsterdam).

Ralph L.J.G. Maassen: The Merits of Videolaryngoscopy during Glottic Visualisation for Endotracheal Intubation. Supervisors: Prof.dr. M. Marcus / Prof.dr. A. van Zundert (University of Queensland).

Maria J. de Sousa Guerreiro: The role of sensory modality in age-related distraction. Supervisor: Prof.dr. C.M. van Heugten; Co-Supervisor: Dr. P.W.M. van Gerven.

Ine Rayen: Effects of developmental fluoxetine exposure on neurobehavioral outcomes. Supervisor: Prof.dr.H.W.M. Steinbusch; Co-Supervisors: Dr.J.L.Pawluski/Dr.T.D.Charlier (Ohio University, USA).

Nynke M.G. Bodde: Psychogenic non-epileptic seizures; a separate disorder or part of a continuum? Supervisors: Prof.dr. R. van Oostenbrugge / Prof.dr. K. Vonck (UZ Gent); Co-Supervisors: Dr. R. Lazeron / Dr. A. de Louw (Epilepsiecentrum Kempenhaeghe, Heeze).

Alejandro M. Gomez: Novel strategies for making myasthenia less gravis: targeting plasma cells and the neuromuscular junction. Supervisor: Prof.dr. M.H. De Baets; Co-Supervisors: Dr. M. Losen / Dr. P. Martinez-Martinez.

Mohammad S. Rahnama'i: Prostaglandins and Phosphodiesterases in the Urinary Bladder Wall. Supervisors: Prof.dr. Ph. Van Kerrebroeck / Prof.dr. S. de Wachter (Universiteit Antwerpen); Co-Supervisor: Dr. G. van Koeveringe.

Mariken B. de Koning: Studying biomarkers in populations at genetic and clinical high risk for psychosis. Supervisors: Prof.dr. T. Amelsvoort / Prof.dr. J. Booij (AMC).

Fabien Boulle: Epigenetic regulation of $\mathrm{BDNF} /$ TrkB signaling in the pathophysiology and treatment of mood disorders. Supervisors: Prof.dr. H.W.M. Steinbusch /

Prof.dr. L. Lanfumey (Universiteit Parijs); Co-Supervisors: Dr. D. van den Hove /

Dr. G. Kenis.

\section{4}

Iris Nowak-Maes: Tinnitus; assessment of quality of life \& cost-effectiveness. Supervisors: Prof.dr. M. Peters / Prof.dr. B. Kremer; Co-Supervisors: Dr. M. Joore / Dr. L. Anteunis.

Marjolein Huijts: Cognitive function in patients with cerebral small vessel disease. Supervisor: Prof.dr. R.J. van Oostenbrugge; Co-Supervisors: Dr. A.A. Duits / Dr. J. Staals.

Markus Gantert: Fetal inflammatory injury as origin of long term disease: Lessons from animal models. Supervisors: Prof.dr. B. Kramer / Prof.dr. L. Zimmermann; Co-Supervisor: Dr. A. Gavilanes.

Elke Kuypers: Fetal development after antenatal exposures: Chorioamnionitis and maternal glucocorticoids. Supervisors: Prof.dr. B.W. Kramer / Prof.dr. H.W. Steinbusch / Prof.dr. Suhas G. Kallapur (University of Cincinnati, Ohio, USA).

Pieter Kubben: Ultra low-field strength intraoperative MRI for Glioblastoma Surgery. Supervisor: Prof.dr. J.J. van Overbeeke; Co-Supervisor: Dr. H. van Santbrink.

Laura Baijens: Surface electrical stimulation of the neck for oropharyngeal dysphagia in Parkinson's disease: therapeutic aspects and reliability of measurement. Supervisor: Prof.dr. B. Kremer; CoSupervisor: Dr. R. Speyer, Townsville.

Janneke Hoeijmakers: Small fiber neuropathy and sodium channels; a paradigm shift. Supervisor: Prof.dr. R.J. van Oostenbrugge; Co-Supervisors: Dr.C.G. Faber/Dr. I.S.J. Merkies.

Stephanie Vos: The Role of biomarkers in preclinical and prodromal Alzheimer's disease. Supervisor: Prof.dr. F.R. Verhey; Co-Supervisor: Dr. P.J. Visser.

Muriel Doors: The Value of Optical Coherence Tomography in Anterior Segment Surgery. Supervisors: Prof.dr. R.M. Nuijts / Prof.dr. C.A. Webers; Co-Supervisor: Dr. T.T.J.M. Berendschot.

Anneke Maas: Sleep problems in individuals with genetic disorders associated with intellectual disability. Supervisors: Prof.dr. I. Curfs / Prof.dr. R. Didden. 
Sebastiaan van Gorp: Translational research on spinal cord injury and cell-based therapies; a focus on pain and sensorimotor disturbances. Supervisors: Prof.dr. B. Joosten / Prof.dr. M. van Kleef; Co-Supervisors: Dr. J. Patijn /Dr. R. Deumens, KU Leuven.

Andrea Sannia: High risk newborns and brain biochemical monitoring. Supervisor: Prof.dr. J.S.H. Vles; Co-Supervisors: Dr.D.Gazzolo, Alessandria, Italy /Dr. A.W.D. Gavilanes.

Julie A.D.A. Dela Cruz: Dopamine mechanisms in learning and memory: Evidence from rodent studies. Supervisors: Prof.dr. H.W.M. Steinbusch / Prof.dr. R.J. Bodnar, New York; Co-Supervisor: Dr. B.P.F. Rutten.

René Besseling: Brain wiring and neuronal dynamics; advances in MR imaging of focal epilepsy. Supervisors: Prof.dr. A.P. Aldenkamp / Prof.dr.ir. W.H. Backes; Co-Supervisor: dr. J.F.A. Jansen.

Maria Quint-Fens: Long-term care after stroke; development and evaluation of a long- term intervention in primary care. Supervisors: Prof.dr. J.F.M. Metsemakers /Prof.dr. C.M. van Heugten / Prof.dr. M. Limburg, Almere; Co-Supervisor: dr. G.H.M.I. Beusmans.

Veronique Moulaert: Life after survival of a cardiac arrest; the heart of the matter. Supervisors: Prof.dr. J.A. Verbunt / Prof.dr. C.M. van Heugten / Prof.dr. D.T. Wade, Oxford, UK.

Feikje Smeets: The hallucinatory-delusional state: a crucial connection in the psychosis symptom network. Supervisor: Prof.dr. J. van Os; Co-Supervisor: Dr. T. Lataster.

Lies Clerx: Alzheimer's disease through the MReye; novel diagnostic markers and the road to clinical implementation". Supervisor: Prof.dr. F. Verhey; Co-Supervisors: Dr. P.J. Visser / P. Aalten.

Sonny Tan: The subthalamic nucleus in Parkinson's disease. Supervisors: Prof.dr. Y. Temel / Prof.dr. H.W.M. Steinbusch / Prof.dr. T. Sharp, Oxford, UK / Prof.dr. V. Visser-Vandewalle, Koln.

Koen van Boxem: The use of pulsed radiofrequency in the management of chronic lumbosacral radicular pain. Supervisors: Prof.dr. M. van Kleef / Prof.dr. E.A.J. Joosten; Co-Supervisor: Assoc. Prof.dr. J. van Zundert.

Jérôme Waterval: Hyperostosis cranialis interna. Supervisors: Prof.dr. J.J. Manni / Prof.dr. R.J. Stokroos.
Sylvie Kolfschoten-van der Kruijs: Psychogenic non-epileptic seizures; the identification of neurophysiological correlates. Supervisors: Prof.dr. A.P. Aldenkamp /Prof.dr. K.E.J. Vonck, Universiteit Gent; Co-Supervisors: Dr. J.F.A. Jansen /Dr. R.H.C. Lazeron, Kempenhaeghe.

Wouter Pluijms: Spinal cord stimulation and pain relief in painful diabetic: polyneuropathy, a translational approach. Supervisors: Prof.dr. M. van Kleef / Prof.dr. E.A. Joosten; Co-supervisor: Dr. C.G. Faber.

Ron Handels: Health technology assessment of diagnostic strategies for Alzheimer's disease. Supervisors: Prof.dr. F.R.J. Verhey / Prof.dr. J.L. Severens (EUR); Co-Supervisor: Dr. M.A. Joore / Dr. C.A.G. Wolfs.

Evelyn Peelen: Regulatory T cells in the pathogenesis of Multiple Sclerosis: potential targets for vitamin D therapy. Supervisors: Prof.dr. R.M.M. Hupperts / Prof.dr. J.W. Cohen Tervaert; Co-Supervisor: Dr. J.G.M.C. Damoiseaux / Dr. M.M.G.L. Thewissen, Diepenbeek.

Reint Jellema: Cell-based therapy for hypoxic-ischemic injury in the preterm brain. Supervisors: Prof.dr. B.W.W. Kramer / Prof.dr. H.W.M. Steinbusch; Co-Supervisor: Dr. W.T.V. Germeraad / Dr. P. Andriessen, Veldhoven.

Maria Wertli: Prognosis of Chronic Clinical Pain Conditions: The Example of Complex Regional Pain Syndrome 1 and Low Back Pain. Supervisors: Prof.dr. M. van Kleef; Co-Supervisor: Dr. F. Brunner, Zürich / Dr. R. Perez, VUmc.

Dagmar Zeef: An experimental model of Huntington's disease: Validation \& Stimulation. Supervisors: Prof.dr. Y. Temel / Prof.dr. H.W.M. Steinbusch; Co-supervisor: Dr. A. Jahanshahi.

Jeroen Decoster: Breaking Down Schizophrenia into phenes, genes and environment. Supervisors: Prof.dr. I. Myin-Germeys / Prof.dr. M. De Hert, KU Leuven; Co-Supervisor: Dr. R. van Winkel.

Eaja Anindya Sekhar Mukherjee: Fetal Alcohol Spectrum Disorders: exploring prevention and management. Supervisor: Prof.dr. L.M.G. Curfs; Co-Supervisor: Prof. S. Hollins, St. George's University of London, UK.

Catherine van Zelst: Inside out; On stereotype awareness, childhood trauma and stigma in psychosis. Supervisors: Prof.dr. Ph. Delespaul / Prof.dr. J. van Os. 
Ibrahim Tolga Binbay: Extended Psychosis Phenotype in the Wider Social Environment. Supervisor: Prof.dr. J. van Os; Co-Supervisor: Dr. M. Drukker.

Frank Van Dael: OCD matters in psychosis. Supervisors: Prof.dr. J. van Os / Prof.dr. I. Myin-Germeys.

Pamela Kleikers: NOXious oxidative stress: from head to toe and back. Supervisors: Prof.dr. H.H.H.W. Schmidt / Prof.dr. H.W.M. Steinbusch; Co-Supervisor: Dr. B. Janssen.

José Luis Gerardo Nava: In vitro assay systems in the development of therapeutic interventions strategies for neuroprotection and repair. Supervisors: Prof.dr.med. J. Weis / Prof.dr. H.W.M. Steinbusch; Co-Supervisor: Dr. G.A. Brook, RWTH Aachen.

Eva Bollen: Cyclic nucleotide signaling and plasticity. Supervisors: Prof.dr. H.W.M. Steinbusch / Prof.dr. R. D’Hooge, KU Leuven; Co-Supervisor: Dr. J. Prickaerts.

\section{5}

Jessica A. Hartmann: A good laugh and a long sleep; Insights from prospective and ambulatory assessments about the importance of positive affect and sleep in mental health. Supervisor: Prof.dr. J. van Os; Co-Supervisors: C.J.P. Simons / Dr. M. Wichers.

Bart Ament: Frailty in old age; conceptualization and care innovations. Supervisors: Prof.dr. G.I.J.M. Kempen / Prof.dr. F.R.J. Verhey; Co-Supervisor: Dr. M.E. de Vugt.

Mayke Janssens: Exploring course and outcome across the psychosis-continuum. Supervisor: Prof.dr. I. Myin-Germeys; Co-Supervisor: Dr. T. Lataster.

Dennis M.J. Hernau: Dopayours is not dopamine: genetic, environmental and pathological variations in dopaminergic stress processing. Supervisor: Prof.dr. I. Myin- Germeys; Co-Supervisors: Prof.dr. F.M. Mottaghy / Dr. D. Collip.

Ingrid M.H. Brands: The adaptation process after acquired brain injury Pieces of the puzzle. Supervisors: Prof.dr. C.M. van Heugten / Prof.dr. D.T. Wade, Oxford UK; Co-Supervisors: Dr. S.Z. Stapert / Dr. S. Kohler.
Francesco Risso: Urinary and salivary S100B monitoring in high risk infants. Supervisor: Prof.dr. J.S.H. Vles; Co-Supervisors: Dr. D. Gazzolo, Genoa, Italy / Dr. A.W.D. Gavilanes.

Alessandro Borghesi: Stem and Progenitor Cells in Preterm Infants: Role in the Pathogenesis and Potential for Therapy. Supervisor: Prof.dr. L. Zimmermann; Prof.dr. B. Kramer; Co-Supervisors: Dr. D. Gazzolo, Genoa, Italy / Dr. A.W.D. Gavilanes.

Claudia Menne-Lothmann: Affect dynamics; A focus on genes, stress, and an opportunity for change. Supervisor: Prof.dr. J. van Os; Co-Supervisors: Dr. M. Wichers / Dr. N. Jacobs.

Martine van Nierop: Surviving childhood new perspectives on the link between childhood trauma and psychosis. Supervisors: Prof.dr. I. Myin-Germeys / Prof.dr. J. van Os; Co-Supervisor: Dr. R. van Winkel.

Sylvia Klinkenberg: VNS in children; more than just seizure reduction. Supervisors: Prof.dr. J. Vles / Prof.dr. A. Aldenkamp; Co-Supervisor: Dr. H. Majoie.

Anouk Linssen: Considerations in designing an adult hearing screening programme. Supervisor: Prof.dr. B. Kremer; Co-Supervisors: Dr. L. Anteunis / Dr. M. Joore.

Janny Hof: Hearing loss in young children; challenges in assessment and intervention. Supervisors: Prof.dr. B. Kremer / Prof.dr. R. Stokroos / Prof.dr. P. van Dijk, RUG; Co-Supervisor: Dr. L. Antheunis.

Kimberly Cox-Limpens: Mechanisms of endogenous brain protection; Clues from the transcriptome. Supervisors: Prof.dr. J. Vles / Prof.dr. L. Zimmermann; Co-Supervisor: Dr. A. Gavilanes.

Els Vanhoutte: Peripheral Neuropathy outcome measures; Standardisation (PeriNomS) study part 2: Getting consensus. Supervisors: Prof.dr. C. Faber / Prof.dr. P. van Doorn; Co-Supervisor: Dr. I. Merkies, Spaarne ziekenhuis Hoofddorp.

Mayienne Bakkers: Small fibers, big troubles; diagnosis and implications of small fiber neuropathy. Supervisors: Prof.dr. C. Faber / Prof.dr. M. de Baets; Co-Supervisor: Dr. I. Merkies, Spaarne ziekenhuis Hoofddorp. 
Ingrid Kramer: Zooming into the micro-level of experience: An approach for understanding and treating psychopathology. Supervisor: Prof.dr. J. van Os; Co-Supervisors: Dr. M. Wichers, UMC Groningen / Dr. C. Simons.

Esther Bouman: Risks and Benefits of Regional Anesthesia in the Perioperative Setting. Supervisors: Prof.dr. M. van Kleef / Prof.dr. M. Marcus, HMC, Qatar / Prof.dr. E. Joosten; Co-Supervisor: Dr. H. Gramke.

Mark Janssen: Selective stimulation of the subthalamic nucleus in Parkinson's disease; dream or near future. Supervisors: Prof.dr. Y. Temel / Prof.dr. V. Visser-Vandewalle, Keulen / Prof.dr. A. Benazzouz, Bordeax, France.

Reina de Kinderen: Health Technology Assessment in Epilepsy; economic evaluations and preference studies. Supervisors: Prof.dr. S. Evers / Prof.dr. A. Aldenkamp; Co-Supervisor: Dr. H. Majoie / Dr. D. Postulart, GGZ O-Brabant.

Saskia Ebus: Interictal epileptiform activity as a marker for clinical outcome. Supervisors: Prof.dr. A. Aldenkamp / Prof.dr. J. Arends, TUE / Prof.dr. P. Boon, Universiteit Gent, België.

Inge Knuts: Experimental and clinical studies into determinants of panic severity. Supervisor: Prof.dr. I. Myin-Germeys; Co-Supervisor: Dr. K. Schruers; Influencing panic.

Nienke Tielemans: Proactive coping post stroke: The Restored4Stroke Self-Management study. Supervisors: Prof.dr. C. van Heugten / Prof.dr. J. Visser-Meily, UMC Utrecht; Co-Supervisor: Dr. V. Schepers, UMC Utrecht.

Tom van Zundert: Improvements Towards Safer Extraglottic Airway Devices. Supervisors: Prof.dr. A.E.M. Marcus / Prof.dr. W. Buhre / Prof.dr. J.R. Brimacombe, Queensland, Australia / Prof.dr. C.A. Hagberg.

Tijmen van Assen: Anterior Cutaneous Nerve Entrapment Syndrome Epidemiology and surgical management. Supervisors: Prof.dr. G.L. Beets / Prof.dr. M. van Kleef / Dr. R.M.H. Roumen / Dr. M.R.M. Scheltinga, MMC Veldhoven.

Rohit Shetty: Understanding the Clinical, Immunological and Genetic Molecular Mechanisms of Keratoconus. Supervisors: Prof.dr. R.M.M.A. Nuijts / Prof.dr. C.A.B. Webers.
Christine van der Leeuw: Blood, bones and brains; peripheral biological endophenotypes and their structural cerebral correlates in psychotic disorder. Supervisor: Prof.dr. J. van Os; Co-supervisor: Dr. M. Marcelis.

Sanne Peeters: The Idle Mind Never Rests; functional brain connectivity across the psychosis continuum. Supervisor: Prof.dr. J. van Os; Co-supervisor: dr. M. Marcelis.

Nick van Goethem: $\alpha 7$ nicotinic acetylcholine receptors and memory processes: mechanistic and behavioral studies. Supervisor: Prof.dr. H.W.M. Steinbusch; Co- supervisor: Dr. J. Prickaerts.

Nicole Leibold: A Breath of fear; a translational approach into the mechanisms of panic. Supervisor: Prof.dr. H.W.M. Steinbusch; Co-supervisors: Dr. K.R.J. Schruers / Dr. D.L.A. van den Hove.

Renske Hamel: The course of mild cognitive impairment and the role of comorbidity. Supervisor: Prof.dr. F.R.J. Verhey; Co-supervisors: Dr. I.H.G.B. Ramakers / Dr. P.J. Visser.

Lucia Speth: Effects of botulinum toxin A injections and bimanual task-oriented therapy on hand functions and bimanual activities in unilateral Cerebral Palsy. Supervisors: Prof.dr. J. Vles; Prof.dr. R. Smeets; Co-supervisor: Dr. Y. JanssenPotten, Adelante Hoensbroek.

Yuan Tian: The effects of Lutein on the inflammatory pathways in age-related macular degeneration (AMD). Supervisors: Prof.dr. C. Webers; Prof.dr. A. Kijlstra, WUR; Co- supervisor: Dr. M. Spreeuwenberg; Dr. H. Tange.

Peggy Spauwen: Cognition and Type 2 diabetes; the interplay of risk factors. Supervisors: Prof.dr. F. Verhey; Prof.dr. C. Stehouwer; Co-supervisor: Dr. M. van Boxtel

Marc Hilhorst: Crescentic glomerulonephritis in ANCA associated vasculitis. Supervisors: Prof.dr. J. Cohen-Tervaert; Co-supervisor: Dr. P. van Paassen

Martin Gevonden: The odd one out: exploring the nature of the association between minority status and psychosis. Supervisors: Prof.dr. J-P. Selten; Prof.dr. J. Booij, Uva; Prof.dr. I. Myin-Germeys

Bart Biallosterski: Structural and functional aspects of sensory-motor Interaction in the urinary bladder. Supervisors: Prof.dr. Ph. Van Kerrebroeck; Prof.dr. S. De Wachter, UvAntwerpen; Co-supervisors: Dr. G. van Koeveringe; Dr. M. Rahnama'i. 
Alexandra König: The use of information and communication technologies (ICT) for the assessment of patients with Alzheimer's Disease and related disorders. Supervisors: prof.dr. F. Verhey; prof.dr. Ph. Robert, Nice, Fr; Co-supervisors: dr. P. Aalten; dr. R. David, Nice. Fr.

Michelene Chenault: Assessing Readiness for Hearing Rehabilitation. Supervisors: prof.dr. M.P.F. Berger; prof.dr. B. Kremer; Co-supervisor: dr. L.J.C. Anteunis.

Anand Vinekar: Retinopathy of Prematurity. Recent advances in tele-medicine screening, risk factors and spectral domain optical coherence tomography imaging. Supervisor: prof.dr. C.A.B. Webers; Co-supervisor: dr. N.J. Bauer

Fleur van Dooren: Diabetes and Depression: exploring the Interface between Pathophysiological and Psychological factors. Supervisors: prof.dr. F.R.J. Verhey; prof.dr. J.K.L. Denollet, UvT; prof.dr. F. Pouwer, UvT; Co-supervisor: dr. M.T. Schram.

Gabrièlla Pons van Dijk: Taekwondo and physical fitness components in middle-aged healthy volunteers; the Sekwondo study. Supervisors: prof.dr. J. Lodder; prof.dr. H. Kingma; Co-supervisor: dr. A.F. Lenssen.

Yara Pujol López: Development and psychoneuroimmunological mechanisms in depression. Supervisor: prof.dr. H.W.M. Steinbusch; Co-supervisors: Dr. G. Kenis; Dr. D. van den Hove; Dr. Aye Mu Myint, München.

Romina Gentier: $\mathrm{UBB}^{+1}$; an important switch in the onset of Alzheimer's disease. Supervisor: prof. fr. H.W.M. Steinbusch; Co-supervisors: Dr. G. Kenis; Dr. D. van den Hove; Dr. Aye Mu Myint, München.

Sanne Smeets: Insights into insight: studies on awareness of deficits after acquired brain injury. Supervisor: Prof. C. van Heugten; Prof. R. Ponds; Co-supervisor: Dr. I. Winkens

Kim Beerhorst: Bone disease in chronic epilepsy: fit for a fracture. Supervisor: Prof. A. Aldenkamp; Prof. R. van Oostenbrugge; Co-supervisor: Dr. P. Verschuure.

Alex Zwanenburg: Cerebral and cardiac signal monitoring in fetal sheep with hypoxic- ischemic encephalopathy. Supervisor: Prof. T. Delhaas; Prof. B. Kramer; Co-supervisors: Dr. T. Wolfs; Dr. P. Andriessen, MMC.
Ismail Sinan Guloksuz: Biological mechanisms of environmental stressors

in psychiatry. Supervisor: Prof. J. van Os; Co-supervisors: Dr. B. Rutten; Dr. M. Drukker.

Seyed Ehsan Pishva MD: Environmental Epigenetics in mental health and illness. Supervisor: Prof.dr. J. van Os; Co-supervisors: Dr. B.P.F. Rutten; Dr. G. Kenis.

Ankie Hamaekers: Rescue ventilation using expiratory ventilation assistance; innovating while clutching at straws. Supervisors: Prof.dr. W.F. Buhre; Prof.dr. M. van Kleef.

Rens Evers. 22q11.2 deletion syndrome: intelligence, psychopathology and neurochemistry at adult age. Supervisors: Prof.dr. L.M.G. Curfs; Prof.dr. T. v. Amelsvoort.

Sarah-Anna Hescham. Novel insights towards memory restoration. Supervisor: Prof.dr. Y. Temel; Co-supervisor: Dr. A. Blokland; Dr. A. Jahanshahi.

João P. da Costa Alvares Viegas Nunes. Insulin receptor sensitization improves affective pathology in various mouse models. Supervisor: Prof.dr. H.W.M. Steinbusch; Co- supervisors: Dr. K-P. Lesch; Dr. T. Strekalova; Dr.B.H. Cline, Oxford.

Yanny Ying-Yee Cheng. Clinical Outcomes After Innovative Lamellar Corneal Transplantation Surgery. Supervisor: Prof.dr. R.M.M.A. Nuijts; Co-supervisor: Dr. J.S.A.G. Schouten.

\section{6}

Oliver Gerlach. Parkinson's disease, deterioration during hospitalization. Supervisor: Prof.dr. R. van Oostenbrugge; Co-supervisor: Dr. W. Weber.

Remo Arts. Intracochlear electrical stimulation to suppress tinnitus. Supervisor: Prof.dr. R.J. Stokroos; Co-supervisor: Dr. E.L.J. Georg.

Mitchel van Eeden. The $€-$ Restore4stroke study: Economic evaluation of stroke care in the Netherlands. Supervisors: Prof.dr.mr. S.M.A.A. Evers; Prof.dr. C.M. v. Heugten; Co-supervisor: dr. G.A.P. van Mastrigt.

Pim Klarenbeek. Blood pressure and cerebral small vessel disease. Supervisor: Prof.dr. R.J. van Oostenbrugge; Co-supervisor: Dr. J. Staals.

Ramona Hohnen. Peripheral pharmacological targets to modify bladder contractility. Supervisor: Prof.dr. Ph.E.V. van Kerrebroeck; Co-supervisors: Dr. G.A. van Koeveringe; Dr. M.A. Sahnama'i; Dr. C. Meriaux. 
Ersoy Kocabicak. Deep brain stimulation of the subthalamic nucleus: Clinical and scientific aspects. Supervisors: Prof.dr. Y. Temel; Prof.dr. K. van Overbeeke; Co-supervisor: Dr. A. Jahanshahi.

Sven Akkerman. Temporal aspects of cyclic messenger signaling in object recognition memory; a pharmalogical approach. Supervisor: Prof.dr. H.W.M. Steinbusch; Co-supervisors: dr. J. Prickaerts; dr. A. Blokland.

Anja Moonen. Emotion and Cognition in Parkinson's disease; etiology and neurobiological mechanisms. Supervisor: Prof.dr. F.R.J. Verhey; Co-supervisor: dr. A.F.G. Leentjens.

Anna Schuith. Three-dimensional bladder tissue morphology. Supervisors: Prof.dr. G.A. van Koeveringe; Prof.dr. M. v. Zandvoort, Aachen; Prof.dr. Ph. V. Kerrebroeck.

Elisabeth van der Ven. Ethnic minority position as risk indicator for autism- Spectrum and psychotic disorders. Supervisors: Prof.dr. J.P. Selten; Prof.dr. J. van Os.

Zuzana Kasanova. Environmental reactivity for better or worse; The impact of stress and reward on neurochemistry, affect and behavior across the psychosis continuum. Supervisor: Prof.dr. I. Myin-Germeys, KU Leuven/UM; Co-supervisor: dr. D. Collip.

Danielle Lambrechts. Ketogenic diet therapies; treatment for children and adults with refractory epilepsy. Supervisors: Prof.dr. H.J.M. Majoie; Prof.dr. J.S.H. Vles; Prof.dr. A.P. Aldenkamp; Cosupervisor: dr. A.J.A. de Louw, Kempenhaghe, Heeze.

Frank van Bussel. Advanced MRI in diabetes; cerebral biomarkers of cognitive decrements. Supervisors: Prof.dr.ir. W.H. Backes; Prof.dr. P.A.M. Hofman; Co-supervisor: dr. J.F.A. Jansen.

Lisa Schönfeldt. Neurostimulation to treat brain injury? Supervisors: Prof.dr. Y. Temel; Prof.dr. S. Hendrikx, Hasselt; Co-supervisor: dr. A. Jahanshahi.

Rianne Geerlings. Transition in patients with childhood-onset epilepsy; a long way to adulthood. Supervisor: Prof.dr. A.P. Aldenkamp; Cosupervisors: dr. A.J.A. de Louw, dr. L.M.C. Gottmer, Kempenhaeghe.
Nele Claes. B cells as multifactorial players in multiple sclerosis pathogenesis: insights from therapeutics. Supervisors: Prof.dr. V. Somers, Hasselt; Prof.dr. R. Hupperts Co-supervisors: Prof.dr. P. Stinissen, dr. J. Fraussen, Hasselt.

Olaf Schijns. Epilepsy surgery and biomarkers from history to molecular imaging. Supervisors: Prof.dr. J.J. van Overbeeke; Prof.dr. H. Clustermann, Aachen; Co-supervisors: dr. G. Hoogland; dr. M.J.P. v. Kroonenburgh.

Lizzy Boots. Balanced and Prepared; development and evaluation of a supportive e- health intervention for caregivers of people with early-stage dementia. Supervisors: Prof.dr. F.R.J. Verhey; Prof.dr. G.I.J.M. Kempen; Co-supervisor: dr. M.E. de Vugt.

Wouter Donders. Towards patient-specific (cerebro-) vascular model applications. Supervisors: Prof.dr. T. Delhaas; Prof.dr.ir. F.N. van de Vosse, TUE; Co-supervisor: dr.ir. W. Huberts.

Sizzle Vanterpool. The implications of intrauterine invasion by microbes for placental Pathology and the occurrence of adverse pregnancy outcomes. Supervisor: Prof.dr. B.W. Kramer. Co-supervisors: dr. J.V. Been, Erasmus MC Rotterdam, dr. U von Rango.

Manuela Heins. The Relationship between Social Adversity, Psychosis, and Depression across an Individual's Life Span. Supervisor: Prof.dr. I. Myin-Germeys.

Christianus van Ganzewinkel. NEONATAL PAIN; Out of Sight, Out of Mind? Supervisor: Prof.dr. B.W.W. Kramer; Co-supervisor: dr. P. Andriessen, MMC Veldhoven.

Anne-Hilde Muris. Hype or hope? Vitamin D in multiple sclerosis; A clinical and immunological perspective. Supervisor: Prof.dr. R.M.M. Hupperts; Co-supervisor: dr. J.G.M.C. Damoiseaux.

Gerard Bode. The link between ceramide transporters, innate Immunity and Alzheimer's disease. Supervisor: Prof.dr. M.H.V. de Baets; Co-supervisors: dr. P. Martinez, dr. M. Losen.

Jo Stevens. Advanced diagnostics and therapeutics for Alzheimer's disease. Supervisor: Prof.dr. M. de Baets; Co-supervisors: dr. M. Losen, dr. P. Martinez-Martinez. 
Rosan Luijcks. Stress and pain in muscles and brain; developing psychophysiological paradigms to examine stress and pain interactions. Supervisors: Prof.dr. J.J. van Os; Prof.dr.ir. H.J. Hermens, UT; Co-supervisor: dr. R. Lousberg.

M.C. Haanschoten. Towards efficient cardiac surgery - the integrating role of anesthesiology and intensive care. Supervisors: Prof. dr. W. Buhre; Prof. dr. A. van Zundert (Queensland); Co-supervisors: Dr. M.A. Soliman Hamad; Dr. A. van Straten (Catharina zkhs.)

Harmen Jan van de Haar. Microvascular and blood-brain barrier dysfunction in Alzheimer's disease. Supervisor: Prof.dr.ir. W. Backes; Prof.dr. F. Verhey; Co-supervisor: Dr. J. Jansen; Dr.ir. M. v. Osch, LUMC.

Coenraad Itz. Chronic low back pain, considerations about: Natural Course, Diagnosis, Interventional Treatment and Costs. Supervisor: Prof.dr. M. van Kleef; Prof.dr. F. Huygen, EUR; Co- supervisor: Dr. B. Ramaekers.

Willemijn Jansen. The Path of Alzheimer's disease: from neuropathology to clinic. Supervisor: Prof.dr. F. Verhey; Co-supervisors: Dr. P.J. Visser; Dr. I. Ramakers.

Ligia dos Santos Mendes Lemes Soares. Phosphodiesterase inhibitors: a potential therapeutic approach for ischemic cerebral injury. Supervisor: Prof.dr. H.W.M. Steinbusch; Co-supervisors: Dr. R.M. Weffort de Oliveira, Brazil; Dr. J. Prickaerts

Martijn Broen. Anxiety and depression in Parkinson's disease. Supervisor: Prof.dr. R.J. van Oostenbrugge; Co-supervisors: Dr. A.F.G. Leentjens; Dr. M.L. Kuijf.

Sandra Schipper. Extrasynaptic receptors as a treatment target in epilepsy. Supervisor: Prof.dr. J.H.S. Vles; Co-supervisors: Dr. G. Hoogland; Dr. S. Klinkenberg; Dr. M.W. Aalbers, RUG.

João Casaca Carreira. Making sense of Antisense Oligonucleotides Therapy in Experimental Huntington's disease. Supervisor: Prof.dr. Y. Temel; Co-supervisors: Dr. A. Jahanshahi; Dr. W. van Roon-Mom, LUMC.

Dominique IJff. Trick or Treat? Cognitive side-effects of antiepileptic treatment. Supervisors: Prof.dr. A.P. Aldenkamp; Prof.dr. M. Majoie; Cosupervisors: Dr. J. Jansen; Dr. R. Lazeron, Kempenhaeghe.
Alfredo Ramirez. Neurogenetic approach in neurodegenerative disorders. Supervisors: Prof.dr. B.P.F. Rutten; Prof.dr. H.W.M. Steinbusch; Prof.dr. M.M. Nothen, University of Bonn.

Nienke Visser. Toric Intraocular lenses in cataract surgery. Supervisor: Prof.dr. R.M.M.A. Nuijts; Co-supervisor: Dr. N.J.C. Bauer.

Jakob Burgstaller. Prognostic indicators for patients with degenerative lumbar spinal stenosis. Supervisor: Prof.dr. M. van Kleef; Co-supervisors: Dr. M.M. Wertli, University of Zurich; Dr. H.F. Gramke.

Mark van den Hurk. Neuronal Identity and Maturation: Insights from the Single-Cell Transcriptome. Supervisors: Prof.dr. H.W.M. Steinbusch; Prof.dr. B.P.F. Rutten; Co- supervisors: Dr. G. Kenis; Dr. C. Bardy, Adelaide.

Maria Nikiforou. Prenatal stress and the fetal gut. Potential interventions to prevent adverse outcomes. Supervisors: Prof.dr. B.W. Kramer; Prof.dr. H.W. Steinbusch; Co- supervisor: Dr. T.G. Wolfs.

Janneke Peijnenborgh. Assessment of cognition, time perception, and motivation in children. Supervisors: Prof.dr. J.S.H. Vles; Prof.dr. A.P. Aldenkamp; Co-supervisors: Dr. J. Hendriksen; Dr. P. Hurks.

Joany Millenaar. Young onset dementia; towards a better understanding of care needs and experiences. Supervisors: Prof.dr. F. Verhey; Prof.dr. R. Koopmans, RUN; Co- supervisors: Dr. M. de Vugt; Dr. C. Bakker, RUN.

\section{7}

Adriana Smits. Perinatal factors and hearing outcome. Supervisors: Prof.dr. R.J. Stokroos; Prof.dr. B.W. Kramer; Prof.dr. B. Kremer.

Angela Bouwmans. Transcranial sonography in parkinsonian disorders: clear window or blurred vision. Supervisor: Prof.dr. W.H. Mess; Co-promotores: Dr. W.E.J. Weber; Dr. A.F.G. Leentjens.

Bjön K. Stessel. Patient centred care after day surgery: scope for improvement. Supervisors: Prof.dr. W. Buhre; Prof.dr. B. Joosten. Co-supervisor: Dr. A.H. Gramke. 
Jan Guy Bogaarts. Quantitative EEG and machine learning methods for the detection of epileptic seizures and cerebral asymmetry. Supervisor: Prof.dr. W.M. Mess; Co-supervisor: Dr.ir. J.P.H. Reulen; Dr.ir. E.D. Gommer.

Martin M. Muiller. Pregnancy derived products for treatment of perinatal brain injuries. Supervisors: Prof.dr. B.W.W. Kramer; Prof.dr. D. Surbek, Bern; Co-supervisors: Dr. T. Wolfs; Dr. G. Gavilanes.

Daan Ophelders. Novel treatment strategies for the protection of the preterm brain; Re- balancing inflammation and regeneration. Supervisor: Prof.dr. B. Kramer; Co-supervisor: Dr. T. Wolfs; Dr. R. Jellema.

Rosalie van Knippenberg. Experience sampling in dementia care; an innovative intervention to support caregivers in daily life. Supervisors: Prof.dr. F. Verhey; Prof.dr. R. Ponds; Prof.dr. I. Myin-Germeys, KU Leuven; Co-supervisor: Dr. M. de Vugt.

Claudia Vingerhoets. Investigating neurobiological mechanisms underlying comorbid cognitive symptoms in psychosis and substance use. Supervisors: Prof.dr. T. van Amelsvoort; Prof.dr. J. Booij, UvA; Co-supervisor: Dr. O. Bloemen

Dennis Oerlemans. Evolution of Neuromodulation for Lower Urinary Tract Dysfunction; Past, Present and Future. Supervisors: Prof.dr. Ph. van Kerrebroeck; Prof.dr. G. van Koeveringe. Co-supervisors: Dr. E. Weil; Dr. T. Marcelissen.

Marion Levy. Evaluation of BDNF/TrkB signaling as a common target in the treatment of major depression and Alzheimer's disease. Supervisors: Prof.dr. H. Steinbusch; Prof. L. Lanfumey, Université Paris Descartes, France. Co-supervisors: Dr. G. Kenis; Dr. D. van den Hove.

Patrick Domen. Stay connected: a family-based diffusion imaging study in psychotic disorder. Supervisor: Prof.dr. J. van Os. Co-supervisor: Dr. M. Marcelis

Geor Bakker. Innovative Approaches to Understanding the Neurobiology of Psychosis. Supervisors: Prof.dr. T. van Amelsfoort; Prof.dr. J. Booij, UvA. Co-supervisor: dr. M. Caan, UvA; dr. O. Bloemen.

Wilma Boevink. HEE! Over Herstel, Empowerment en Ervaringsdeskundigheid in de psychiatrie. Supervisors: Prof.dr. J. van Os; Prof.dr. Ph. Delespaul. Co-supervisor: dr. H. Kroon.
Nataliia Markova. Modified swim test as a mouse depression paradigm of enhanced Cognitive processing: the role of GSK3 $\beta$. Supervisor: Prof.dr. H. Steinbusch; Prof.dr. K-P. Lesch, University of Wuerzburg. Co-supervisor: Dr. T. Strekalova.

Merijn van de Laar. Individual differences in insomnia; implications of Psychological factors for diagnosis and treatment. Supervisor: Prof.dr. A. Aldenkamp; Prof.dr. D. Pevernagie, Universiteit Gent. Co-supervisor: Dr. S. Overeem, TUE.

Willem Buskermolen. If only I could tell ...; Measuring predictors for challenging behaviour in people with both intellectual disability and hearing impairment. Supervisor: Prof.dr. A. Aldenkamp. Co-supervisor: Dr. J. Hoekman, UL.

Kay Deckers. The role of lifestyle factors in primary prevention of dementia; an epidemiological perspective. Supervisor: Prof.dr. F. Verhey. Cosupervisor: Dr. M. van Boxtel; Dr. S. Kohler.

Brechje Dandachi-FitzGerald. Symptom validity in clinical assessments. Supervisors: Prof.dr. R. Ponds; Prof.dr. F. Verhey.

Maurice Theunissen. Understanding factors affecting postoperative Quality of Life. Supervisors: Prof.dr. M. Peters, Prof.dr. M. Marcus. Co-supervisor: Dr. H. Gramke.

Anna Cleutjens. COgnitive-Pulmonary Disease? Neuropsychological functioning in patients with COPD. Supervisors: Prof.dr. E. Wouters, Prof.dr. R. Ponds. Co-supervisors: Dr. D. Janssen, Horn, Dr. J. Dijkstra.

Laura Serpero. Next Generaton Biomarkers in Perinatal Medicine: S100B Protein. Supervisors: Prof.dr. D. Gazzalo, Alessandria, Italy; Prof.dr. B.W.W. Kramer. Co-supervisor: Dr. A.W.D. Gavilanes.

Alessandro Varrica. S100B Protein and Congential Heart Diseases: Brain Aspects. Supervisors: Prof.dr. D. Gazzalo, Alessandria, Italy; Prof.dr. J.S.H. Vles; Prof.dr. L.J.I. Zimmermann. Co-supervisor: Dr. A.W.D. Gavilanes.

Pim R.A. Heckman. Targeting phosphodiesterase type 4 for improving cognitive fronto- striatal function: a translational approach. Supervisor: Prof.dr. J.G. Ramaekers. Co- supervisors: Dr. J.H.H.J. Prickaerts; Dr. A. Blokland.

Sven van Poucke. Platelets, form sample to big data; exploring granularity in platelet research. Supervisors: Prof.dr. M.A.E. Marcus; Prof.dr. W. Buhre. Co-supervisor: Dr. M. Lancé. 
Désirée M.J. Vrijens. Dysfunctions of the Lower Urinary Tract and Affective Symptoms. Supervisors: Prof.dr. Ph.E.V. van Kerrebroeck; Prof.dr. G.A. van Koeveringe. Co- supervisors: Dr. C. Leue.

Tamar van Veenendaal. Neurotransmitters \& Networks. An MR view on epilepsy and antiepileptic drugs. Supervisors: Prof.dr.ir. W.H. Backes; Prof.dr. A.P. Aldenkamp. Co- supervisor: Dr. J.F.A. Jansen.

Evelien M. Barendse. Autism Spectrum Disorders in High Functioning Adolescents; Diagnostic considerations (AHA). Supervisors: Prof.dr. A.P. Aldenkamp;

Prof.dr. R.P.C. Kessels, Radboud University.

Roy Lardenoije. A venture into the epigenetics of aging and Alzheimer's Disease. Supervisors: Prof.dr. B.P.F. Rutten; Prof.dr. H.W.M. Steinbusch. Co-supervisors: Dr. D. van den Hove; Dr. C.A. Lemere, USA.

Charlotte L. Mentzel. The course recognition and treatment of movement disorders in severe mental illness. Supervisors: Prof.dr. P.N. van Harten; Prof.dr. M.A.J. de Koning- Tijssen, UMCG. Cosupervisor: Dr. P.R. Bakker.

Tim Batink. Third Wave Behaviour Therapy: Process Measures and Contextual Interventions. Supervisors: Prof.dr. F.P.M.L. Peeters; Prof.dr. J.J. van Os; Prof.dr. M.C. Wichers, UMC Groningen.

Kevin L.J. Rademakers. Detrusor Underactivity: From Theory to Clinical Assessment. Supervisors: Prof.dr. G.A. van Koeveringe; Prof.dr. Ph.E.V.van Kerrebroeck. Co-supervisor: Dr. M. Oelke.

Iris M.J. Lange. Should I stay or should I go? Brain mechanisms underlying fear and safety learning, and exposure therapy outcome. Supervisors: Prof.dr. K.R.J. Schruers; Prof.dr. T.A.M.J. van Amelsfoort. Co-supervisor: Dr. L. Goossens.

Ruben G.F. Hendriksen. Evidence for a dystrophin-associated encephalopathy in Duchenne Muscular Dystrophy. Supervisor: Prof.dr. J.S.H. Vles. Co-supervisors: Dr. G. Hoogland; Dr. M.W. Aalbers, UMC Groningen.

Michael Gofeld. Strengths and limitations of the lumbar spine ultrasound-guided interventions. Supervisor: Prof.dr. M. van Kleef. Co-supervisor: Dr. M. Sommer.
Willem A.R. Zwaans. Strategies for chronic inguinal pain. Supervisor: Prof.dr. M. van Kleef. Co-supervisors: Dr. R.H.M. Roumen; Dr. M.R.M. Scheltinga, MMC Veldhoven.

Linda M. Rolf. Mapping the effects of vitamin D in multiple sclerosis A 3D Perspective. Supervisor: Prof.dr. R.M.M. Hupperts. Co-supervisors: Dr. J.G.M.C. Damoiseaux; Dr. J.J.F.M. Smolders, CWZ Nijmegen.

Maarten van Beek. Spinal Cord Stimulation in Clinical and Experimental Painful Diabetic Polyneuropathy. Supervisors: Prof.dr. E.A. Joosten; Prof.dr. M. van Kleef. Co- supervisor: Dr. S.M.J. van Kuijk.

Melina Barkhuizen. Genetic and perinatal risk factors for movement disorders. Supervisors: prof.dr. B.W.W. Kramer, prof.dr. H.W.M. Steinbusch, Prof.dr. A.F. Grobler. Co- supervisor: dr. A.W.D. Gavilanes-Jimenez.

Renske Uiterwijk. Cognitive function and cerebral small vessel disease in hypertension. Supervisor: prof.dr. R.J. van Oostenbrugge. Co-supervisor: Dr. J.E.A. Staals.

Elles Douven. Depression and apathy after stroke. Supervisor: prof.dr. F.R.J. Verhey. Co- supervisors: Dr. P. Aalten, dr. J. Staals.

Mauro Pessia. Brain K+ Channels: from molecular and physiological features to autism spectrum disorder and intellectual disability. Supervisors: prof.dr. H.W.M. Steinbusch, prof.dr. M.B. Donati, It.

Carsten Leue. Hyperarousal in the Hospital and what to do about it: the MED-PSYCH- NET - a transitional network approach fostering personalized care in psychosomatic medicine. Supervisors: Prof.dr. J. van Os, Prof.dr. A. Masclee. Co-supervisors: Dr. J. Strik, Dr. J. Kruimel

Andrea S. Herrera Soto. Aminochrome, an endotoxin for inducing a new rat model of Parkinson's Disease. Supervisor: prof.dr. H.W.M. Steinbusch. Co-supervisors: Prof.dr. Juan Segura-Aquilar; prof. G. Diaz-Veliz, Santiago of Chile

Eline E.B. de Clerck. Ocular neurodegenerative changes and macular cysts in prediabetes and type 2 diabetes. Supervisors: Prof.dr. C.A.B. Webers, Prof.dr. C.D.A. Stehouwer. Co-supervisor: Dr. J.S.A.G. Schouten 
Steven T.H. Honings. Exploring psychosis and multidirectional violence: a prospective study in the general population. Supervisor: Prof.dr. J. van Os. Co-supervisor: Dr. M. Drukker

\section{8}

Sau May Wong. Advances in Microvasculair MRI Techniques: Breaking the Pathophysiological Barriers in Cerebral Small Vessel Disease. Supervisor: Prof.drir. W.H. Backes, Porf.dr. R.J. van Oostenbrugge. Co-supervisor: Dr. J.F.A. Jansen

Mark B.N. van Winkel. Lonely at heart and stressed in company of Others; the influence of daily life social experiences and emotions on depression. Supervisors: prof.dr. F. Peeters; prof.dr. I. Myin-Germeys, KU Leuven/UM; prof.dr. M. Wichers, UMC Groningen

Harsha Birur Laxmana Rao. Revisiting the vascular theory of glaucoma using optical coherence tomography angiography. Supervisors: prof.dr. C.A.B. Webers; prof.dr. R.N. Weinreb, University of California, San Diego

Babette L.R. Reijs. Cognitive correlates of cerebrospinal fluid biomarkers for Alzheimer's disease. Supervisor: prof.dr. F.R.J. Verhey. Co-supervisors: Dr. P.J. Visser; dr. I.H.G.B. Ramakers

Rachel Slangen. Spinal cord stimulation in painful diabetic peripheral Neuropathy. Clinical- and cost-effectiveness. Supervisors: prof.dr. M. van Kleef; Prof.dr. C. Dirksen; prof.dr. C. Faber

Ganne Chaitanya. Epilepsy: A network disorder. Supervisors: prof.dr. A.P. Aldenkamp; prof. P. Satishchandra, NIMHANS, Bangalore, India. Cosupervisors: Dr. J.F.A. Jansen; Dr. S. Zinger, TUE

Sumitha Rajendrarao. New Insight into the Multifaceted Pathogenic Mechanisms of Sporadic Amyotrophic Lateral Sclerosis. Supervisors: prof.dr. B.W. Kamer; prof.dr. H.W. Steinbusch. Co-supervisor: prof. T.R. Raju, NIMHANS, Bangalore, India

Suzanne Roggeveen. Interference of mobile phone with electrophysiology and emotions; results from short-term experimental studies. Supervisor: Prof.dr. J. van Os. Co-supervisor: Dr. R. Lousberg.
Matthias Walter. Multi-methodological approaches to investigate lower urinary tract function in health and disease. Supervisors: Prof.dr. Ph.E.V.A. van Kerrebroek; Prof.dr. G.A. van Koeveringe; Prof.dr. A. Curt, Zürich, CH.

Lalit Gupta. Inhomogeneities in spontaneous brain fluctuations. Supervisors: Prof.dr.ir. WH. Backes; Prof.dr. P.A.M. Hofman. Co-supervisor: Dr. J.F.A. Jansen.

Chaitra Jayadev. Impact of imaging the pediatric retina. Supervisor: Prof.dr. C.A.B. Webers. Co-supervisor: Dr. N.J.C. Bauer; Dr. A. Vinekar.

Annelie Klippel. Navigating through complexity; processes and mechanisms underlying the development of psychosis. Supervisors: Prof.dr. I. Myin-Germeys, KU-Leuven; Prof.dr. M.C. Wichers, UMC Groningen. Co-supervisor: Dr. U. Reininghaus.

Kürssat Altinbas, Reconstructing the Diagnostic Framework of Bipolarity. Supervisor: Prof.dr. J. van Os. Co-supervisor: Dr. I.S. Guiloksüz.

Andrea J.R. Balthasar. Eyes of the needle; Spectral tissue sensing, an innovative technology for detecting various tissue types during percutaneous needle-based procedures in locoregional anesthesia and pain medicine. Supervisor: Prof.dr. M. van Kleef. Co-supervisor: Dr. G-J. van Geffen, Radboud UMC Nijmegen.

Walmari Pilz. Shedding light on oropharyngeal dysphagia in myotonic dystrophy type 1. Supervisor: Prof.dr. B. Kremer. Co-supervisors: Dr. L.W.J. Baijens; Dr. V. Lima Passos.

Whitney M. Freeze, Microvascular contributions to dementia. Supervisors: Prof. dr. F.R.J. Verhey, Prof. dr. ir. W.H. Backes, Co-supervisor: Dr. H.I.L. Jacobs

Syenna H.J. Schievink, Vascular cognitive impairment: at the heart of the matter. Supervisors: Prof. dr. F.J.R. Verhey, Prof. dr. R.J. van Oostenbrugge, Co-supervisors: Dr. S. Köhler 